\title{
Claus Reis
}

\section{Kommunales Integrationsmanagement}

Leitfaden für die Praxis 
Claus Reis

\section{Kommunales \\ Integrationsmanagement}

\section{Leitfaden für die Praxis}

\section{Mit Beiträgen von}

Heiner Brülle, David Hübner und Benedikt Siebenhaar

unter Mitarbeit von

Andreas Borchers, Susan Geideck, Tina Hobusch, Simon Klein,

Christian Kolbe, Martina Schu, Beate Seusing und Lutz Wende

\section{Nomos}


Die Deutsche Nationalbibliothek verzeichnet diese Publikation in der Deutschen Nationalbibliografie; detaillierte bibliografische Daten sind im Internet über http://dnb.d-nb.de abrufbar.

1. Auflage 2020

(c) Claus Reis

Publiziert von

Nomos Verlagsgesellschaft mbH \& Co. KG

Waldseestraße 3-5 | 76530 Baden-Baden

www.nomos.de

Gesamtherstellung:

Nomos Verlagsgesellschaft mbH \& Co. KG

Waldseestraße 3-5 | 76530 Baden-Baden

ISBN (Print): 978-3-8487-7628-3

ISBN (ePDF): 978-3-7489-1011-4

DOI: https://doi.org/10.5771/9783748910114

\section{(9) (1) $\Theta \Theta$}

Dieses Werk ist lizenziert unter einer

Creative Commons Namensnennung - Nicht kommerziell Keine Bearbeitungen 4.0 International Lizenz.

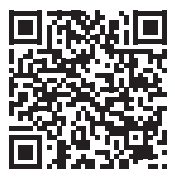

Onlineversion Nomos eLibrary 


\section{Inhaltsverzeichnis}

1 Einleitung 11

2 Gelingensbedingungen für Produktionsnetzwerke - Erfahrungen aus dem Pilotprojekt „Einwanderung gestalten NRW“

2.1 Die Ausgangslage: Integration als Teilhabe 15

2.2 Ziele und Erfolgskriterien 16

$\begin{array}{ll}2.3 \text { Gelingensbedingungen } & 16\end{array}$

3 Theoretische Vorüberlegungen zum Integrationsmanagement 21

3.1 Integration und Teilhabe 22

3.1.1 „Integration“ - rechtlich und sozialwissenschaftlich verstanden

3.1.1.1 Zum Begriff „Integration“ im Migrationsrecht 22

3.1.1.2 Sozialwissenschaftliche Anmerkungen zum Begriff „Integration“ 25

3.1.1.3 Integration als handlungsoffener langfristiger Prozess

3.1.2 „Teilhabe“ als konzeptionelle Basis des Integrationsmanagements

3.1.2.1 Teilhabe als rechts- und sozialwissenschaftlicher Leitbegriff

3.1.2.2 „Teilhabe“ aus der Perspektive des Capability Approach

3.2 Teilhabe und Zuwanderung

3.2.1 Teilhabe und das Feld sozialer Hilfen

3.2.2 Das Feld der Zuwanderungspolitik - erste Annäherungen

3.2.3 Diskursformationen im Feld der Zuwanderungspolitik

3.2.4 Exkurs: Der Begriff „Integration“ aus

diskurstheoretischer Perspektive

3.2.5 Der „Brückenschlag“ zum Feld sozialer Hilfe(n) 
3.3 Ein komplexes Verständnis von „Teilhabe“ als konzeptionelle Grundlage des Integrationsmanagements

4 Ideen zur Gestaltung und zum Management multilateraler Kooperationsstrukturen (Produktionsnetzwerke) 65

4.1 Vorbemerkung 65

4.2 Kooperation und Netzwerke 66

4.2.1 Begriffliche Klärungen 66

4.2.2 Netzwerktypen $\quad 71$

4.2.2.1 Hierarchische und heterarchische Netzwerke $\quad 71$

4.2.2.2 Richtungsoffene und zielgerichtete Netzwerke $\quad 73$

4.3 Theoretische Zugänge zum Verständnis von Netzwerken $\quad 76$

4.3.1 Ein strukturtheoretischer Zugang zu Netzwerken $\quad 77$

4.3.1.1 Die Grundproblematik von organisationalen Netzwerken 78

4.3.1.2 Vertrauen, Verlässlichkeit und Reziprozität $\quad 79$

4.3.2 Ein sozialpsychologischer Zugang zu Netzwerken $\quad 84$

4.3.3 Ein dienstleistungstheoretischer Zugang zu

Netzwerken 86

4.4 Wie entstehen Produktionsnetzwerke? 90

4.4.1 Die Problemstellung 90

4.4.2 Die Kooperation sozialer

Dienstleistungsorganisationen als Anforderung im

Arbeitsalltag 92

4.4.2.1 „Verteilte Expertise“ 92

4.4.2.2 „Fälle“ als Zugänge zu geteilten Realitäten 95

4.4.2.3 Was ist der „Fall“? 97

4.4.2.4 Der „Fall“ als das „Allgemeine im Besonderen“ 101

4.4.3 Die Planung von Leistungsprozessen 103

4.5 Gelingensbedingungen für die Gestaltung multilateraler

Kooperationsstrukturen in Produktionsnetzwerken $\quad 107$

4.6 Methodisches I: Wie werden „Produktionsnetzwerke“ geknüpft? 111

4.6.1 Vorentscheidungen und Vorbereitungen 113

4.6.1.1 Konzept 113

4.6.1.2 Methoden 119

4.6.1.2.1 Kartenabfrage $\quad 119$

4.6.1.2.2 Beteiligtenanalyse $\quad 119$ 
4.6.2 Planungskonferenzen

4.6.2.1 Erarbeitung integrierter Fallrekonstruktionen

4.6.2.1.1 Konzept

4.6.2.1.2 Methode

4.6.2.2 Feststellung "generativer Themen“

4.6.2.2.1 Konzept

4.6.2.2.2 Methode

4.6.2.3 Die Entwicklung eines Zielsystems

4.6.2.3.1 Konzept

4.6.2.3.2 Methode

4.6.2.4 Ablauf einer Planungskonferenz und Entwicklung von Zielen

4.6.3 Umsetzung des Zielsystems

4.6.3.1.1 Dienstleistungsketten

4.6.3.1.2 Standards und Verfahren

4.6.3.1.3 Schnittstellen-, Kommunikations-

4.6.3.2 Produkte und Abstimmungsregelungen

4.6.3.2.1 Neue und gemeinsame Angebote

4.6.3.2.2 Fallkonferenzen

4.6.3.2.3 Angebotsüberblick

4.6.3.2.4 Projekte

4.6.3.2.5 Systematischer

Informationsaustausch

4.6.3.2.6 Workshops mit der operativen Ebene und Zwischenworkshops

4.6.3.2.7 Kooperationsvereinbarungen

4.6.4 Verstetigung und Überprüfung

4.6.4.2 Monitoring und Dokumentation

4.6.4.3 Überprüfung und Reflexion der

Dienstleistungsketten

4.6.5 Zusammenfassung

4.7 Methodisches II: Organisationsübergreifende

Dienstleistungsketten als Instrument und Ergebnis von

Produktionsnetzwerken

4.7.1 Das Konzept der „Dienstleistungskette“

4.7.1.1 Prozessorganisation 
4.7.1.2 Organisationsübergreifende

Dienstleistungsketten $\quad 156$

4.7.2 Visualisierungen 159

4.7.2.1 Grundsätzliches 159

4.7.2.2 Hinweise zur praktischen Gestaltung $\quad 160$

4.7.3 Dienstleistungsketten und integrierte Leistungen $\quad 163$

4.8 Methodisches III: Wie werden Netzwerke gesteuert? 164

4.8.1 Management als Institution und als Funktion $\quad 165$

4.8.2 Funktionen des Netzwerkmanagements 166

4.8.2.1 Selektion: Auswahl der Netzwerkpartnerinnen und -partner 166

4.8.2.2 Allokation: Verteilung der Aufgaben in der Dienstleistungskette 168

4.8.2.3 Regulation: Festlegung von Regeln und Strukturen 169

4.8.2.4 Evaluation: Auswertung und Bewertung der gemeinsamen Arbeit

4.9 Ein Beispiel: Planungsprozesse am Standort Z 171

4.9.1 Vorbemerkung 171

4.9.2 Standort Z - von Fällen zu Zielen 172

5 Anregungen zur Ausgestaltung von Case Management im Rahmen des kommunalen Integrationsmanagements $\quad 187$

$\begin{array}{ll}5.1 \text { Vorbemerkung } & 187\end{array}$

5.2 Case Management - erste Definitionen 188

5.3 Kontext und Konzepte von Case Management 192

5.3.1 Case Management - ein Konzept und seine Varianten 192 5.3.1.1 „Consumer-driven“ Case Management 194

5.3.1.2 „System-driven“ Case Management 194

5.3.2 Der Kontext des Case Managements und seine Analyse 196 5.3.2.1 Zielgruppen 196

5.3.2.2 Politische Einbettung 201

5.3.2.3 Innerorganisatorische Voraussetzungen für

Case Management 
5.3.2.4 Außerorganisatorische Voraussetzungen für Case Management in der Variante des rechtskreisübergreifenden Case Managements

5.3.2.4.1 Rechtskreisübergreifendes Case Management bedarf einer besonderen Indikation

5.3.2.4.2 Rechtskreisübergreifendes Case Management ist häufig nicht das einzige Angebot

5.3.2.4.3 Integrationsmanagement ist rechtskreisübergreifendes Case Management

5.3.3 Konzeptionelle Vorentscheidungen zum Case Management

5.3.3.1 Der Auftrag

5.3.3.2 Der Zuschnitt der Interaktionsbeziehung 210

5.3.3.3 Funktionale Ausgestaltung 211

5.3.3.4 Qualifikation und Kompetenz des Personals 214

5.3.3.5 Angebotssteuerung

5.3.3.6 Zusammenfassung

5.4 Ein komplexes Konzept von Case Management

5.4.1 Erstberatung

5.4.1.1 Konzeptionelle Überlegungen zur Indikation von Case Management

5.4.1.1.1 Zielgruppe

5.4.1.2 Organisatorische Überlegungen zur Indikation von Case Management

$\begin{array}{ll}\text { 5.4.3 Zielvereinbarung } & 228\end{array}$

$\begin{array}{ll}\text { 5.4.4 Hilfeplanung } & 231\end{array}$

$\begin{array}{ll}\text { 5.4.5 Leistungssteuerung } & 233\end{array}$

5.4.6 Monitoring der Leistungserbringung 237

5.4.7 Abschluss und Auswertung (Evaluation) 239

5.5 Interaktion im Rahmen des Case Managements 241

5.5.1 Offene Gespräche 243

5.5.2 Gespräche, in denen die Fallmanager eine steuernde Funktion übernehmen 
5.5.3 „Abfragen“ im Rahmen des Assessments

5.5.4 Scheiternde Arbeitsbündnisse

5.5.5 Fazit

5.6 Ausblick 252

5.7 Beispiele aus den Projektkommunen 254

6 Fachliche Anforderungen an ein Monitoring des

Integrationsmanagements für Geflüchtete und Neuzugewanderte 257

6.1 Vorbemerkung 257

6.2 Das Integrationsmonitoring Nordrhein-Westfalen 259

6.3 Teilhabe und Integration als Prozess sozialer

Dienstleistungen

6.4 Wesentliche Felder sozialer Hilfen für das

Integrationsmanagement für Geflüchtete und

Neuzugewanderte

$\begin{array}{lll}7 & \text { Literatur } & 271\end{array}$

$\begin{array}{ll}\text { Anhang } & 285\end{array}$ 


\section{Einleitung}

Die große Zahl Menschen, die 2015 und 2016 nach Deutschland zugewandert ist, darf nicht dazu verführen, diese als homogene Gruppe anzusehen. Der Forschungsbereich beim Sachverständigenrat deutscher Stiftungen für Integration und Migration bringt dies deutlich zum Ausdruck: „Den Flüchtling gibt es nicht, dafür sind die (früheren und aktuellen) Lebenslagen der Menschen zu verschieden. Entsprechend kommt es bei der Aufnahme und Versorgung sowie bei Maßnahmen zur Förderung der gesellschaftlichen Teilhabe darauf an, wie gut sie zu den individuellen Lebenslagen passen" (Forschungsbereich 2017: S. 6). Angekommen sind Individuen mit je eigenen Biografien, Erfahrungen und Kompetenzen, die auf ihnen mehr oder weniger unbekannte gesellschaftliche Strukturen und Prozesse stoßen. Sie müssen sich orientieren, sich in unterschiedlichsten Lebensbereichen zurechtfinden. Dabei benötigen sie eine Unterstützung, die der Individualität ihrer Lebenslage gerecht wird: „Ansprechpartner als Lotsen geben Orientierung: Flüchtlinge benötigen sowohl Informationen als auch Hilfe zur Selbsthilfe. Deshalb ist es besonders in der Anfangszeit wichtig, dass sie zentrale Ansprechpartner haben. Deutlich wird vor allem die wichtige Rolle des sozialen Fachpersonals, in den Blick zu nehmen ist aber auch die mitunter zu dezentrale Unterstützungsstruktur für Flüchtlinge in den Kommunen“ (ebd.: S. 8). Dies gilt nicht nur für geflüchtete Menschen, sondern für viele, die aus unterschiedlichsten Gründen nach Deutschland zugewandert sind.

Bei der Gestaltung von Einwanderung auf kommunaler Ebene muss diese Ausgangslage berücksichtigt werden. Es müssen Prozesse definiert werden, die dazu dienen, den Zugewanderten die Leistungen zukommen zu lassen, die ihnen zustehen - und zwar unabhängig vom jeweiligen aufenthaltsrechtlichen Status. Diese Aufgabe erfordert einerseits die Entwicklung der Steuerung eines Dienstleistungsangebots für den Einzelfall, andererseits - um dies adäquat zu gewährleisten - den Aufbau von Kooperationsstrukturen im Bereich „helfender“ Institutionen. Das im Einzelfall notwendige Angebot kann nur institutions- und rechtskreisübergreifend realisiert werden, was die Kooperation vieler Akteure auf lokaler Ebene ebenso voraussetzt wie gemeinsam getragene Fachplanungen.

Ein vom Land Nordrhein-Westfalen von 2017 bis 2019 durchgeführtes Modellprojekt „Einwanderung gestalten Nordrhein-Westfalen“ setzte hier 


\section{Einleitung}

an und unterstützte zwölf Kommunen im Land dabei, einerseits Steuerungsstrukturen auf Netzwerkebene, andererseits ein individuelles Case Management auf der Ebene des Einzelfalles aufzubauen.

Die Kommunen wurden über den gesamten Projektzeitraum von einer wissenschaftlichen Begleitung unterstützt, die eine komplexe Aufgabenstellung verfolgte:

1) Erarbeitung eines Gesamtkonzepts.

2) Beratung der Steuerungsorgane an den einzelnen Projektstandorten im Hinblick auf

- Projektgestaltung,

- Netzwerkaufbau,

- Ausgestaltung der Fallebene.

3) Qualifizierung der Projektkoordinatorinnen und Projektkoordinatoren aus den zwölf Standorten mit den Inhalten:

- Netzwerkanalyse,

- Netzwerkmanagement.

4) Durchführung regelmäßiger Reflexionsveranstaltungen für Koordinatorinnen und Koordinatoren.

5) Durchführung themenbezogener Austausch-Workshops für die zwölf Standorte.

Ein wichtiger Bestandteil der Arbeit der wissenschaftlichen Begleitung war die Erstellung von theoretischen und praktischen Konzepten ${ }^{1}$, die in der Praxis erprobt werden konnten - die Reflexion der hieraus resultierenden Erfahrungen diente dann wiederum der Weiterentwicklung der Konzepte. Der vorliegende Leitfaden stellt insofern das Resultat eines gemeinsamen Lernprozesses von wissenschaftlicher Begleitung und „Praxis“ dar ${ }^{2}$. Er basiert auf Texten und Vortragsmanuskripten, die von Prof. Dr. Reis im Zuge der Projektarbeit erstellt und jetzt durch Beiträge von Heiner Brülle, David Hübner und Benedikt Siebenhaar ergänzt und bereichert wurden. Alle Texte wurden im Team der wissenschaftlichen Begleitung diskutiert und fortgeschrieben. Nach der Modellphase sollen nun die Ergebnisse des Projekts genutzt werden, um in Nordrhein-Westfalen landesweit analoge Strukturen eines „Kommunalen Integrationsmanagements“ auf Netzwerk-

1 Diese basieren auf Erkenntnissen des Teams der wissenschaftlichen Begleitung, die teilweise in der Vergangenheit publiziert wurden (vgl. Reis u.a. 2011, Reis u.a. 2016).

2 Den Kommunen und insbesondere den Projektleitungen, den Koordinatorinnen und Koordinatoren sowie allen weiteren Beteiligten vor Ort sei an dieser Stelle sehr herzlich für ihre Mitarbeit im Projekt gedankt. 
und Einzelfallebene auf- bzw. auszubauen. Da die Erkenntnisse, die im Projekt gewonnen werden konnten, über das Land Nordrhein-Westfalen hinaus von hoher Relevanz sind, haben sich die Autoren zu einer Publikation in Buchform entschlossen. ${ }^{3}$

Eine wichtige Erkenntnis der Modellphase bildet die Notwendigkeit der Implementierung des Handlungskonzeptes Case Management in der von der Fachorganisation Deutsche Gesellschaft für Case und Case Management (DGCC) vertretenen Variante. Diese hebt in ihren „Rahmenempfehlungen zum Handlungskonzept Case Management“ (DGCC 2015) hervor, dass Case Management die Brücke zwischen Einzelfall und Versorgungsstrukturen schlägt, weshalb es mehr ist als eine „Methode“. Die Arbeit mit Personen (Einzelfallorientierung) im Case Management ist nur eingebettet in eine effektive kooperative Ausgestaltung und Verknüpfung von Leistungen und Angeboten auf struktureller Ebene realisierbar (in Form von „Produktionsnetzwerken“). Da Letzteres in den verschiedenen Handlungsfeldern des Sozial- und Gesundheitswesens auf unterschiedliche gesetzliche und institutionelle Voraussetzungen trifft, wird die konkrete Umsetzung des Handlungskonzepts jeweils anders aussehen, es wird immer Varianten des Handlungskonzepts geben, die sich unterscheiden, aber einen gemeinsamen Kern aufweisen.

Der vorliegende Leitfaden dient der Vertiefung einiger in diesem Endbericht dargestellten Inhalte. Er bezieht sich auf die genannten beiden Seiten von Case Management und soll das Konzept konkretisieren und für die praktische Umsetzung „handhabbar" machen.

Im zweiten Kapitel des Leitfadens werden im Rückgriff auf die Ergebnisse des Modellprojekts „Einwanderung gestalten Nordrhein-Westfalen“ dessen Gelingensbedingungen dargestellt und diskutiert, die sich aus der Perspektive der wissenschaftlichen Begleitung über alle zwölf Projektstandorte hinweg gezeigt haben. Diese Gelingensbedingungen verweisen auf die Kombination der Konstitution von Produktionsnetzwerken einerseits (Kapitel 4) und dem Handlungskonzept Case Management andererseits (Kapitel 5). Diese Kombination findet aber im Kontext eines spezifischen, rechtlich und politisch definierten Feldes ${ }^{4}$ statt, dem der Zuwande-

3 Die Ergebnisse des gesamten Modellprojekts werden im parallel erscheinenden Endbericht des Ministeriums für Kinder, Familien, Flüchtlinge und Integration des Landes Nordrhein-Westfalen (MKFFI) veröffentlicht.

4 Der Begriff „Feld“ wird hier wie im gesamten Text nicht umgangssprachlich, sondern in dem spezifischen Sinn verwendet, den ihm Pierre Bourdieu verliehen hat (vgl. hierzu ausführlich Abschnitt 3.2). 
rung. Aus diesem Grund ist es notwendig, zunächst Begriffe und Regeln, die in diesem Feld geläufig sind, kritisch zu hinterfragen (wie z. B. „Integration") und so zu rekonstruieren, dass sie dazu dienen können, dieses Feld theoretisch und konzeptionell zu erschließen (Kapitel 3). Erst auf der Basis dieser grundsätzlichen Überlegungen erschließt sich die Fruchtbarkeit der im vierten und fünften Kapitel ausführlich dargestellten Handlungskonzepte „Produktionsnetzwerk“ und „Case Management“ für die Anwendung im Feld der Zuwanderung. Im sechsten Kapitel werden dann Fragen diskutiert, die mit dem Monitoring der Prozesse des Integrationsmanagements verbunden sind. Das siebte Kapitel enthält ausführliche Literaturhinweise. $^{5}$

5 Darüber hinaus finden sich im vierten Kapitel des Leitfadens sowie im Anhang (Der Anhang ist über folgenden Link abrufbar: https://www.nomos-shop.de/isbn/9 78-3-8487-7628-3). Beispiele aus Standorten des Modellprojekts. Sie haben ausdrücklich exemplarischen Charakter, da die vorgestellten Konzepte und Prozesse stark von lokalen Bedingungen abhängen, die nicht einfach übertragbar sind. 


\section{Gelingensbedingungen für Produktionsnetzwerke - Erfahrungen aus dem Pilotprojekt „Einwanderung gestalten NRW“}

In diesem Kapitel soll versucht werden, zu identifizieren, welche Bedingungen und Faktoren für den erfolgreichen Aufbau und die Stabilisierung von Produktionsnetzwerken im Rahmen des Projekts „Einwanderung gestalten NRW“ verantwortlich waren. Hierzu wird zunächst die Ausgangslage zu Projektbeginn rekapituliert (2.1), aus der sich die Ziele des Projekts ergeben (2.2). Diese Ziele bilden den Maßstab für den Erfolg der Einzelprojekte; mögliche Einflussfaktoren auf den Projekterfolg werden dann in Abschnitt 2.3 vorgestellt.

\subsection{Die Ausgangslage: Integration als Teilhabe}

Das Projekt „Einwanderung gestalten NRW“ steht in engem Zusammenhang mit den Problemstellungen, die mit Integration von Zugewanderten auf kommunaler Ebene zusammenhängen. ${ }^{6}$ Deshalb ergibt sich über die Auslegung des Begriffs „Integration“ die Formulierung von Zielen und konkreten Anforderungen. Wir folgen der Definition von Integration durch den Sachverständigenrat deutscher Stiftungen für Integration und Migration als „,chancengleiche Teilhabe an den zentralen Bereichen des gesellschaftlichen Leben" (Forschungsbereich 2017: S. 11). Hieraus resultiert die Notwendigkeit einer möglichst raschen Einbindung der Zugewanderten in die Regelstrukturen der Leistungen, die auf kommunaler Ebene erbracht werden.

„Teilhabe“ in diesem Sinne bedeutet zweierlei: Die chancengleiche Teilhabe an gesellschaftlichen Feldern (Teilhabe erster Ordnung) und die Teilhabe an den Hilfen, die die Gesellschaft bereitstellt, um diese Teilhabe zu sichern (Teilhabe zweiter Ordnung). Letztere müsste beinhalten, dass einerseits für alle Gesellschaftsmitglieder (objektive) Teilhabechancen eröffnet und andererseits für diejenigen (subjektive) Teilhabekompetenzen gestärkt werden, die diese Chancen nicht ohne Unterstützung wahrnehmen

6 Dies gilt wohl unabhängig von der Unschärfe des Begriffs Integration. 
können (vgl. Abschnitt 3.1). Allerdings spaltet sich das Feld sozialer Hilfe zur Sicherung von Teilhabe in Teilfelder mit je spezifischen Akteuren und Beziehungen, spezifischen Rechtsgrundlagen und Verwaltungsstrukturen, die zwar vielfältige Überlappungen und Schnittstellen aufweisen, häufig aber zu unkoordiniert arbeiten (u. a. Jugendhilfe, Sozialhilfe, Grundsicherung für Arbeitsuchende)(vgl. Abschnitt 3.2).

Die Aufgabenstellung, die sich zur Realisierung der Ziele von „Einwanderung gestalten“ ergibt, beinhaltet somit, diese Spaltungen und „Versäulungen" zu überwinden und Strukturen zu verändern bzw. neu zu schaffen, um Teilhabe zu sichern. Dies gilt nicht nur für Zugewanderte; deshalb sollten „Sondersysteme“ möglichst vermieden werden.

\subsection{Ziele und Erfolgskriterien}

Im Förderaufruf des Landes Nordrhein-Westfalen zum Modellprojekt „Einwanderung gestalten NRW“ wurden dessen Ziele benannt, die die Ausgangslage aufgreifen: „Durch das Modellprojekt soll ein Organisationsentwicklungsprozess in den Kommunen angestoßen werden, der die strategische Zusammenarbeit bei der Wahrnehmung von Aufgaben fördert, die im Kontext der Integration von Zugewanderten entstehen. Es sollen Unterstützungssysteme auf der Grundlage vorhandener lokaler Strukturen und Akteure konzipiert, vernetzt und umgesetzt werden.“

Hieraus lassen sich zwei Erfolgskriterien für das Gesamtprojekt ableiten, die je nach lokaler Konstellation einmal mehr, einmal weniger erreicht werden:

1) Strukturen für einen Organisationsentwicklungsprozess sind geschaffen, der die strategische Zusammenarbeit aller Akteure fördert, die mit der Integration von Zugewanderten zu tun haben (Strukturebene).

2) Auf der lokalen Ebene sind vernetzte Unterstützungssysteme für die Zugewanderten geschaffen (Einzelfallebene).

\subsection{Gelingensbedingungen}

Im Folgenden sollen auf der Grundlage einer vergleichenden qualitativen Betrachtung die Bedingungen benannt werden, die die Erreichung der beiden genannten Ziele besonders fördern. Die vergleichende Betrachtung basiert auf der Dokumentation der Entwicklungen an den zwölf Projektstandorten in Form von Protokollen der Projektgremien, Zwischenberich- 
ten an die Bezirksregierung Arnsberg, Konzepten und Power-Point-Präsentationen. ${ }^{7}$

Die zentralen Gelingensbedingungen sind:

- Einbindung aller relevanten kommunalen Akteure. Um das Ziel der Einbindung in Regelstrukturen realisieren zu können, müssen alle für die Realisierung von Teilhabe relevanten kommunalen Akteure am Produktionsnetzwerk beteiligt werden und darin aktiv mitarbeiten (vgl. Reis u. a. 2011; Reis u. a. 2016). Das bedeutet, dass das Netzwerk zwangsläufig „rechtskreisübergreifend“ konstituiert werden muss, also neben Ausländerbehörde und Sozialamt je nach konkretem Zuschnitt des Vorhabens auch Jobcenter, Jugendamt, Wohnungsamt etc. mitarbeiten. Bei „Einwanderung gestalten NRW“ zeigte sich deutlich, dass eine breite Einbindung unterschiedlicher Behörden ein zentraler Erfolgsfaktor war - erst die aktive Mitarbeit schaffte die Bereitschaft zur Öffnung von Strukturen und Prozessen.

- Einbeziehung der zentralen Akteure und Akteursgruppen außerhalb der Verwaltung. Im Feld der Zuwanderung operieren nicht nur kommunale Akteure, sondern z. B. auch Verbände der freien Wohlfahrtspflege, ehrenamtlich Tätige, Migrantenselbstorganisationen, Bildungsträger, Vertretungen der Wirtschaft und Betriebe. Sie sind wichtige Netzwerkpartner, da sie einerseits selbst (Dienst-)Leistungen erbringen (z. B. Beratung und Begleitung), zum anderen aber auch die Netzwerkaktivitäten in der Stadtgesellschaft verankern. Auch wenn die $\mathrm{Zu}$ sammenarbeit nicht immer konfliktfrei verlief und manchmal Skepsis bei den kommunalen Akteuren zu erkennen war, gelang die Zielerreichung an den Projektstandorten, an denen ein breites Spektrum extrakommunaler Akteure am Netzwerk beteiligt war, am besten.

- Eigenständige Projektorganisation. Die meisten Kommunen entschlossen sich, für die Durchführung des Projekts eine eigene Projektorganisation aufzubauen. Einige wenige verzichteten darauf, da es bereits zentrale Gremien zum Thema „Migration“ bzw. „Integration“ gab, die sich dann zusätzlich mit „Einwanderung gestalten“ beschäftigten. Im Projektverlauf zeigte sich jedoch, dass projektspezifische Lenkungsgruppen als Entscheidungsorgane flexibler und auf das Projekt hin fokussierter waren. Die Standorte mit dem höchsten Grad an Zielerreichung hatten alle eine eigene Projektorganisation. Diese umfasste Lenkungs- und

7 Diese Betrachtung ersetzt nicht die Evaluation des Projekts, die auf einer anderen Datengrundlage aufbaut (Interviews, Gruppendiskussionen) und basiert auf dem Stand der Einzelprojekte am 30.6.2019, also sechs Monate vor Projektende. 
Projektgruppen mit der Beteiligung von Mitarbeitenden auf unterschiedlichen Hierarchieebenen. Wichtig ist bei derartigen Projekten grundsätzlich, dass die Projektarbeit darin mündet, neue oder veränderte Strukturen und Prozesse nachhaltig in das Regelgeschehen zu implementieren.

- Ansiedlung des Projekts auf hoher Hierarchieebene. Da das Projekt darauf abzielt, dass Strukturen und Prozesse innerhalb und zwischen bestehenden Organisationen verändert werden, sind von Anfang an, zumindest aber zum Projektende entsprechende politische und administrative Entscheidungen zu treffen. Dies gelingt nur bei klaren Entscheidungsstrukturen in der Projektorganisation und einer entsprechenden Unterstützung durch wichtige, d. h. mächtige „Entscheider“ in Politik und Verwaltung. Die Unterstützung wird dokumentiert durch eine entsprechende Anbindung des Projekts auf einer hohen Hierarchieebene. Der Zusammenhang zwischen Anbindung und Projekterfolg war augenfällig.

- Klare Entscheidungsstrukturen und Rollenverteilungen in der Projektorganisation. In allen Projekten waren sowohl Leitungskräfte wie operativ Tätige in verschiedenen Konstellationen (Lenkungsgruppe, Projektgruppen) tätig. Als sehr wichtig erwies sich, dass die damit verbundenen Rollen bereits zu Projektbeginn geklärt und entsprechend Entscheidungsstrukturen und -wege definiert waren. Dies erleichterte die Kommunikation nach innen sowie die Transparenz und Legitimation nach außen.

- Verbindlichkeit der Beschlüsse. Klare Entscheidungsstrukturen befördern auch die Verbindlichkeit der Beschlüsse - dieser Zusammenhang konnte eindeutig belegt werden; und dass die Verbindlichkeit ein wesentlicher Faktor für den Erfolg der Netzwerke war, ist so folgerichtig wie evident.

- Kompetenzen der Koordinatorinnen und Koordinatoren. Die Projektkoordinatorinnen und -koordinatoren waren die tragende Säule der lokalen Projektorganisationen. Wie die Rückschau zeigt, war ihre Kompetenz im doppelten Sinne (Können wie administrative Macht) entscheidend für den Projekterfolg. Sie mussten in der Lage sein, Projektmanagement zu konzipieren und zu realisieren, mussten Netzwerke moderieren, für Monitoring sorgen und in Krisensituationen agieren hierzu benötigten sie entsprechende Qualifikationen (die z. B. im Rahmen des Projekts in einer Weiterbildungsreihe vermittelt wurden) und auch - projektinterne - Entscheidungskompetenzen. Als sehr wichtig 
erwies sich die Besetzung der Koordinationsstellen mit erfahrenen Mitarbeitenden aus der Verwaltung.

- Netzwerkaufbau über „Fälle“. Die Konstitution von Netzwerken wird durch die Schaffung eines gemeinsamen, möglichst konkreten Problemverständnisses erleichtert. Hierzu hat sich die gemeinsame Diskussion von konkreten Fällen als besonders geeignet erwiesen. Über die Darstellung und Diskussion unterschiedlicher Perspektiven auf einen Fall konnte sich ein gemeinsames Fallverständnis als Basis der weiteren Arbeit im Netzwerk entwickeln. Fast alle Einzelprojekte griffen (in unterschiedlicher Weise) auf Fallbezug zurück.

- Einbeziehung der Nutzerperspektive. Im Laufe der Arbeit entwickelten einige Standorte Ansätze, um die Betroffenen, d. h. Zugewanderte als potenzielle Nutzerinnen und Nutzer von Leistungsangeboten über den Fallbezug (s. o.) hinaus explizit in den Prozess einzubeziehen (z. B. Workshops, Begleitgruppen). Auf diese Weise konnten die im Zuge der Netzwerkarbeit entwickelten Angebote zu den konkreten Bedarfslagen der potenziellen Nutzerinnen und Nutzern rückgekoppelt werden.

- Verknüpfung zwischen Einzelfall- und Strukturebene. Im Zuge der Entwicklung der Einzelprojekte griffen mehrere Projektstandorte das Konzept des Case Managements auf und etablierten ein entsprechendes Leistungsangebot. Auf diese Weise konnten die Fortschritte in der Kooperation auf der Strukturebene für die Arbeit mit den Betroffenen im Einzelfall genutzt werden. So wurde die Netzwerkarbeit „geerdet“, umgekehrt konnten Erkenntnisse aus der Einzelfallarbeit für die Arbeit der Netzwerke genutzt werden, was sozialplanerische Aktivitäten tendenziell stützt.

- Entwicklung eines träger- und rechtskreisübergreifenden Case Management-Konzeptes. Case Management im Feld der Zuwanderung ist zwangsläufig rechtskreisübergreifend (s. o.). Gleichzeitig gibt es auf der lokalen Ebene eine Reihe von Akteuren, die ebenfalls nach dem Handlungskonzept Case Management arbeiten (z. B. Jugendmigrationsdienste, Jobcenter). Um hier Reibungsverluste durch Konkurrenz zu vermeiden, ist es notwendig, ein trägerübergreifendes Konzept zu entwickeln, das die jeweiligen Rollen und Steuerungsfunktionen klärt. Bestandteil des Konzepts ist ein Monitoring, das die Grundlage für die Weiterentwicklung des trägerübergreifenden Angebots bildet.

- Dokumentation des Gesamtprozesses. Nicht nur Case ManagementProzesse müssen dokumentiert werden, sondern grundsätzlich der Gesamtprozess, d. h. auch die Netzwerkaktivitäten. Dies erleichtert die Kontinuität, da die Geschichte des Netzwerks verfügbar bleibt, und si- 
chert die Transparenz nach außen. Gerade die erfolgreichsten Einzelprojekte stützten sich auf eine zeitnahe und komplexe Dokumentation.

- Hohe Transparenz gegenüber Politik, Verwaltung und Stadtgesellschaft. Transparenz ist wichtig, um die Akzeptanz der Projektarbeit auf breiter Basis herzustellen und auch darüber die Verbindlichkeit von Ergebnissen abzusichern.

- Systematische Reflexion der Entwicklungen. Ein so komplexer Prozess, wie er durch „Einwanderung gestalten NRW“ angestoßen wurde, bedarf einer systematischen Reflexion durch alle Beteiligten. Im Projekt wurden Gelegenheiten für eine solche Reflexion geschaffen, z. B. durch die Etablierung externer Beratung, die durch die wissenschaftliche Begleitung sichergestellt wurde. In der Rückschau zeigt sich die hohe Bedeutung des Beratungsangebots für den Projekterfolg.

- Flankierung auf Landesebene. Das Land stellte mit der wissenschaftlichen Begleitung und ihren Angeboten einen wesentlichen Erfolgsfaktor, da diese sowohl für die Weiterbildung der Koordinatorenstellen wie auch als Garant für eine systematische Reflexion verantwortlich war. Gleichzeitig stellten begleitende Workshops und Arbeitsgruppen sowie ein Weiterbildungsprogramm für Case Managerinnen und Manager, die vom Land initiiert und finanziert wurden, einen wichtige Rahmen für das Gesamtprojekt und auch die Einzelprojekte an den Standorten dar. 


\section{Theoretische Vorüberlegungen zum Integrationsmanagement}

Wie in den Kapiteln 4 und 5 gezeigt wird, basiert Integrationsmanagement einerseits auf der Anwendung des Handlungskonzepts "Case Management" in einem bestimmten gesellschaftlichen Feld, dem der Migration (Kapitel 5), andererseits auf der Konstitution von Produktionsnetzwerken, die diese Anwendung erst erfolgreich werden lassen (Kapitel 4). Die Ausführungen in den beiden Kapiteln setzen voraus, dass begrifflich geklärt ist, was unter „Integration“ verstanden werden soll (d. h. was ist „Integrations"management) und dass Konturen und Dynamiken dieses Feldes der Migration hinreichend deutlich sind (d. h. was ist der Gegenstand von Integrations, management“).

Das vorliegende Kapitel soll dazu beitragen, diese beiden Voraussetzungen zu schaffen:

- In Abschnitt 3.1 wird ausführlich dargestellt, dass der Begriff „Integration" bislang sowohl rechtlich wie sozialwissenschaftlich unzureichend geklärt ist und es sich deshalb empfiehlt, für analytische Zwecke eher auf den Begriff „Teilhabe“ zurückzugreifen.

- In Abschnitt 3.2 wird, hieran anknüpfend, gezeigt, dass „Teilhabe“ auf die Konstitution und Dynamik gesellschaftlicher Felder verweist und die Problemstellungen, mit denen Integrationsmanagement in der Praxis konfrontiert ist, nur dann zureichend analysiert und verstanden werden können (als Voraussetzung für manageriales Handeln), wenn diese Dynamik analysiert und verstanden worden ist. 


\subsection{Integration und Teilhabe}

\subsection{1 „Integration“ - rechtlich und sozialwissenschaftlich verstanden ${ }^{8}$}

\subsubsection{Zum Begriff „Integration“ im Migrationsrecht}

Das „Gesetz über den Aufenthalt, die Erwerbstätigkeit und die Integration von Ausländern im Bundesgebiet“ (AufenthG) vom 30.7.2004 ist das erste Gesetz, das ausdrückliche Bestimmungen zur Integration von Ausländerinnen und Ausländern ${ }^{9}$ enthält (vgl. Eichenhofer 2013: S. 109). Mit dem Inkrafttreten dieses Gesetzes am 1.1.2005 begann eine neue Phase der Migrationspolitik. Die Verantwortung für Integrationsförderung war nicht mehr lediglich individueller oder gesellschaftlicher Natur, sie verwandelte sich in eine staatliche Aufgabenstellung (vgl. Groß 2007: S. 316f.). Das Integrationskonzept des Aufenthaltsgesetzes basiert auf „konkret-individuellen Rechtsbeziehungen zwischen dem deutschen Aufnahmestaat und dem einzelnen Ausländer" (Eichenhofer 2013: S. 281). So soll die Integration von rechtmäßig im Bundesgebiet lebenden Ausländerinnen und Ausländern in das wirtschaftliche, kulturelle und gesellschaftliche Leben durch Integrationskurse gefördert werden ( $\$ 43$ Abs. 1 AufenthG). Eine wichtige Konsequenz dieser Neuregelung war, dass der Begriff „Integration“ nicht mehr ausschließlich ein sozial- oder kulturwissenschaftlicher Fachbegriff war, sondern auch ein Rechtsbegriff (vgl. Eichenhofer 2013: S. 110). Allerdings enthalten weder das Aufenthaltsgesetz noch die Rechtsverordnungen oder Verwaltungsvorschriften eine Legaldefinition zum Rechtsbegriff „Integration“. Somit „lässt sich der Begriff der Integration im Aufenthaltsgesetz jedenfalls nicht allgemeinverbindlich bestimmen - weder unter Heranziehung juristischer Auslegungsmethoden, noch durch den Versuch einer Einordnung in soziologische Theorien" (ebd.: S. 273 mit weiteren Nachweisen).

Der Begriff „Integration“ hat einen vielfältigen Niederschlag im aktuellen deutschen Migrationsrecht gefunden“ (Gusy/Müller 2013: S. 270) und in $\mathbb{1} 1$ Abs. 1 AufenthG wird u. a. die Integration von Ausländerinnen und Ausländern in Deutschland als Ziel des Gesetzes genannt. „Noch nie ist

8 Dieser Abschnitt wurde von Benedikt Siebenhaar verfasst.

9 In rechtlichen Kontexten wird stets von „Ausländern“ gesprochen, um den Unterschied zu deutschen Staatsbürgern und Staatsbürgerinnen zu markieren. Im folgenden Text wird dem teilweise gefolgt, allerdings außerhalb der juristischen Diskussion der Begriff „Zugewanderte“ verwendet. 
ein Leitbild des Migrationsrechts derart vielfältig im Gesetz erwähnt wie dasjenige der ,Integration'. Zugleich ist das Konzept dort vielfältig vorausgesetzt, aber wenig konkretisiert“" (ebd.: S. 271) - mit der Konsequenz, dass Inhalt und Zielsetzung von Integration bundesgesetzlich nicht näher bestimmt sind. Das Land Nordrhein-Westfalen hat mit dem „Teilhabe- und Integrationsgesetz NRW“ seit 2012 eine der bundesweit wenigen landesgesetzlichen Regelungen zur Integration von Menschen mit Migrationshintergrund. Aber auch dieses Gesetz enthält für die Integrationsförderung auf der Landesebene keine Legaldefinitionen zu den zentralen Rechtsbegriffen Integration und Teilhabe. Das Gleiche gilt für das am 8.7.2016 verabschiedete Integrationsgesetz auf Bundesebene, ein Artikelgesetz mit zahlreichen Änderungen von Einzelgesetzen. „Trotz seiner ehrgeizigen Bezeichnung beabsichtigt das Integrationsgesetz keinesfalls, umfassend Einfluss auf die gesamtgesellschaftliche Systemintegration oder die Sozialintegration sämtlicher Einwanderer nehmen zu wollen. Vielmehr richtet es sich ausschließlich an Asylbewerber, die im Laufe der letzten zwei Jahre nach Deutschland eingereist sind und eine gute Bleibeperspektive haben" (Eichenhofer 2016: S. 257). Es „enttäuscht freilich schon deshalb, weil es sich in der asyl- und sozialrechtlichen Aufbereitung der Flüchtlingskrise erschöpft und keinen konzeptionellen Neuanfang wagt“" (Thym 2017: S. 206).

Kritisch soll auf die bisherige Engführung der Diskussion zum Verständnis von Integration hingewiesen werden. „Integration ist in den letzten Jahrzehnten in der politischen und rechtswissenschaftlichen Debatte semantisch vorwiegend bis ausschließlich auf Migranten fokussiert worden. Das ist sachlich zu eng gefasst und zudem in mehrfacher Hinsicht irreführend“ (Kluth 2018: S. 47). Denn „Integration (und die Entstehung neuer Vielfalt) primär auf Migranten zu beziehen, erweckt vor diesem Hintergrund den irrigen Eindruck, dass erstens die Mitglieder der aufnehmenden Gesellschaft keinen Integrationsanforderungen oder -pflichten unterliegen, dass zweitens Integration statisch zu verstehen ist, dass drittens die Integration von Migranten ganz andere Instrumente erfordert und führt viertens dazu, dass die Integrationsbedarfe in den übrigen Teilen der Bevölkerung vernachlässigt werden. Es ist vorzugswürdig, Integration wieder stärker als einen gesamtgesellschaftlichen Normalvorgang zu verstehen und entsprechend für die Integration von Migranten soweit wie möglich einen allgemeinen Steuerungsansatz zu wählen und nur dort spezielle Instrumente zu nutzen, wo der allgemeine Steuerungsansatz aufgrund besonderer Ausgangslagen nicht passt, etwa im Bereich des Spracherwerbs“ (ebd.). Somit lässt sich feststellen, dass der angesichts der gesellschaftlichen und 
politischen Entwicklung des letzten Jahrzehnts zentrale Begriff „Integration" zwar formal Eingang in Bundes- und Landesgesetze gefunden hat, aber bislang eine präzise Bestimmung seines Inhaltes und seiner Zielsetzung aussteht. Vielmehr werden mit dem Begriff „Integration“ divergierende Inhalte verknüpft, wie Eichenhofer in seiner Darstellung von Landesintegrationsgesetzen zeigt (vgl. Eichenhofer 2016). Das Bayerische Integrationsgesetz verlagert demnach (in Artikel 1, Satz 2) die Verantwortung für Integration auf die Zugewanderten: „Es ist Ziel dieses Gesetzes, diesen Menschen für die Zeit ihres Aufenthalts Hilfe und Unterstützung anzubieten, um ihnen das Leben in dem ihnen zunächst fremden und unbekannten Land zu erleichtern (Integrationsförderung), sie aber zugleich auf die im Rahmen ihres Gast- und Aufenthaltsstatus unabdingbare Achtung der Leitkultur zu verpflichten und dazu eigene Integrationsanstrengungen abzuverlangen (Integrationspflicht)“ (ebd.: S. 256). Dies kommentiert Leven folgendermaßen: „Das bayerische Integrationsgesetz verpflichtet Geflüchtete auf die Beachtung einer Leitkultur, erwartet also eine kulturelle, mithin ,innere' Anpassung. Damit überschreitet es dem Recht...typischerweise gesetzte Grenzen, da es einen Teil der persönlichen Lebensführung antastet“ (Leven 2018: S. 339f.). Uwe Berlit, Vorsitzender Richter am Bundesverwaltungsgericht stützt diese Position: „Individuelle Integrationsoffenheit, die von vielfältigen sozialen, kulturellen, ökonomischen, politischen und ,sozialpsychologischen' Faktoren abhängig ist, ist aber keine Rechtspflicht für das Individuum. In dem weiten Rahmen des geltenden Rechts dürfen auch integrationsfeindliche Positionen vertreten und integrationsaverse Handlungen begangen werden. Ökonomische Leistungsfähigkeit garantiert zudem nicht individuelle oder gesellschaftliche Integrationsoffenheit" (Berlit 2018a: S. 232). Dem stellt er eine andere Auffassung von Integration entgegen: „Integration als Herstellung von sozialer, gesellschaftlicher und politischer Einheit aus Vielfalt ist aber nicht auf die Bewältigung von Migrationsprozessen zu beschränken und fordert selbst in seiner thematischen Verengung neben den MigrantInnen zudem auch die Aufnahmegesellschaft, die durch Integrationsprozesse verändert wird. Ich folge dem Sachverständigenrat deutscher Stiftungen für Integration und Migration (SVR) und verstehe hier Integration als möglichst chancengleiche Teilhabe aller Personen an den zentralen Bereichen des gesellschaftlichen Lebens, u. a. Teilhabe an Bildung, Ausbildung, Arbeit und den Schutzund Fürsorgesystemen des Rechts und Wohlfahrtsstaates sowie soziale und politische Partizipation“ (Berlit 2018a: S. 231). Analoges findet sich im Integrationsgesetz des Landes Berlin: „Ziel, Menschen mit Migrationshintergrund die Möglichkeit der gleichberechtigten Teilhabe in allen Bereichen 
des gesellschaftlichen Lebens zu geben und gleichzeitig jede Benachteiligung und Bevorzugung gemäß Art. 3 III des GG und Art 10 II der Verfassung von Berlin auszuschließen“ (zitiert nach Eichenhofer 2016: S. 254).

Hier zeigen sich die beiden Pole der Diskussion um „Integration“, die seit 2005 immer wieder hervorgehoben werden: Integration als Bringschuld der Zugewanderten oder Integration als Befähigung zur Teilhabe am gesellschaftlichen Leben. Hierzu schreibt Elena Buck: „In der Bundesrepublik Deutschland ist ,Integration“ parteiübergreifend konsensfähig. Obwohl der Begriff inhaltlich Unterschiedliches bezeichnet, werden die Forderung nach Ermöglichung (,fördern') von Teilhabe sowie die Anforderung (,fordern'), sich anzupassen und auf eine bestimmte Art und Weise aktiv zu beteiligen, als allgegenwärtiger Bestandteil der Integrationsdebatte immer wieder wiederholt“ (Buck 2011: S. 47). Warum mit dem Begriff Unterschiedliches bezeichnet wird und er deshalb unpräzise bleiben muss, wird weiter unten behandelt. Zunächst stellt sich die Frage, ob der Begriff überhaupt weiter verwendet werden sollte (vgl. auch Bommes 2018: S. 102, FN 5). Bevor diese Frage entschieden wird, soll im Folgenden die sozialwissenschaftliche Begriffsverwendung skizziert werden.

\subsubsection{Sozialwissenschaftliche Anmerkungen zum Begriff „Integration“}

Die sozialwissenschaftliche Diskussion um das Phänomen der „Integration" von Gesellschaften und die Auswirkungen von Migration auf diese Integration bezieht sich zu einem großen Teil auf David Lockwoods bekannte Unterscheidung von „Systemintegration“ und „Sozialintegration“. So greift z. B. Esser diese Unterscheidung auf und bezeichnet als System ,jene Form des Zusammenhalts der Teile eines sozialen Systems, die sich unabhängig von den speziellen Motiven und Beziehungen der individuellen Akteure und oft genug auch gegen ihre Absichten und Interessen, sozusagen anonym und hinter ihrem Rücken, ergibt und durchsetzt, während die soziale Integration unmittelbar mit den Motiven, Orientierungen, Absichten und - insbesondere - den Beziehungen der Akteure zu tun hat" (Esser 2001: S. 8ff.; zitiert nach Eichenhofer 2013: S. 216). Dabei handelt es sich allerdings um eine analytische Unterscheidung, die nicht dazu führen darf, die reale Verquickung beider Dimensionen zu verkennen (vgl. hierzu Bommes 2004). Während also mit dem Begriff der „Systemintegration“ der Zusammenhalt der Gesellschaft und ihrer Teilsysteme, somit die Folgen von Zuwanderung auf der Makroebene, thematisiert werden, zielt „Sozialintegration" auf die Beziehungen der individuellen Akteure zueinander so- 
wie zu gesellschaftlichen Teilsystemen und zur Gesellschaft insgesamt (vgl. Gestring 2014a: S. 82). In der öffentlichen Diskussion um die „Integration“ von Zugewanderten werden häufig beide Dimensionen miteinander vermischt: Eine problembehaftete Integration von Individuen wird rasch zur Systemfrage stilisiert, z. B. über die Unterstellung von Ghettobildung als Folge von Zuwanderung. Unabhängig davon stellt sich aber die Frage nach dem Sinn der Unterscheidung. Sie führt die klassische Gegenüberstellung von Individuum und Gesellschaft bzw. Handeln und Struktur auf, die in der modernen Soziologie weitgehend überwunden ist, weiter. Statt an die Tradition der Unterscheidung von System- und Sozialintegration anzuknüpfen, muss ein dynamisches, prozessuales Wechselverhältnis von Struktur und Handeln konzipiert werden. Nach Bommes geht es dabei um zwei Fragestellungen:

„a) Welche Voraussetzungen bringen Migranten mit bzw. müssen sie erfüllen, um zu wichtigen sozialen Bereichen wie Arbeit, Erziehung, Recht oder Gesundheit Zugang zu erhalten und daran teilnehmen zu können? Werden diese Voraussetzungen von ihnen erfüllt?

b) Welche Auswirkungen haben Zuwanderungen auf diese sozialen Bereiche, also Arbeitsmärkte und Unternehmen, Politik und Recht, Schulen oder Krankenhäuser? Unter welchen Voraussetzungen und in welcher Form beziehen diese Migranten ein? Welche strukturellen Folgen hat dies für diese Bereiche? Welche Lebensverhältnisse resultieren daraus für Migranten und ihre Familien sowie ggf. für andere betroffene Bevölkerungsteile sowohl der Zuwandererbevölkerung wie der Aufnahmegesellschaft" (Bommes 2004: S. 36)?

Dies bedeutet, die Verschränkung von individuellem Handeln und strukturellen Gegebenheiten zu berücksichtigen. In diesem Sinne stellte Bommes bereits 2004 in seiner Expertise für den Zuwanderungsrat fest: „Die Analyse von Integrationsprozessen zielt darauf zu erfassen, wie die Handlungs- und Kommunikationskompetenzen, mit denen Migranten ausgestattet sind, und die sozialen Strukturen, an denen sie im Gefolge ihrer Migration im Zuwanderungskontext teilnehmen (Arbeitsmärkte, Wohnungsmärkte, Schulen, Krankenhäuser etc.), aufeinander bezogen bzw. aufeinander beziehbar gemacht werden, so dass soziales Handeln, also Wirtschaften, Erziehen, Behandeln, Entscheiden usw. möglich wird. Dieser Integrationsprozess führt in seinem Fortgang auf Seiten der Migranten zu bestimmten sozialen Lebensverhältnissen und Kompetenzen und auf Seiten der Sozialstrukturen, an denen Migranten teilnehmen, zur Bildung von Potenzialen (z. B. mehr Kindern, internationalen Belegschaften, mehrsprachigen Kompetenzen, kulturellen Pluralisierungen) und Einschrän- 
kungen (z. B. Steigerung von Einkommensdiskrepanzen, Verknappung oder potenzielle Aufzehrung von Kollektivgütern auf Wohnungsmärkten, im Erziehungssystem oder im Gesundheitssystem). In der Analyse dieser Zusammenhänge geht es also darum, Migration und Integration empirisch offen als eine soziale Konstellation begreifbar zu machen, die mit der Entstehung von sozialen Chancen und Restriktionen für soziale Strukturen und die betroffenen Individuen verbunden ist. Die differenzierten gesellschaftlichen Bereiche stellen Gelegenheiten zur Verfügung, die Migranten auf der Basis ihres sozialen und kulturellen Kapitals ergreifen. Diese Prozesse lösen wiederum Strukturveränderungen in diesen Bereichen aus, die zur Voraussetzung für das Gelingen oder Misslingen weiterer Migrationsund Integrationsprozesse werden. Es handelt sich also um komplexe interdependente Zusammenhänge, die durch einseitige Erklärungsansätze nicht angemessen zu erfassen sind“ (ebd.: S. 34).

\subsubsection{Integration als handlungsoffener langfristiger Prozess}

Der damit in den Vordergrund gerückte Prozesscharakter von Integration spielt auch in den rechtswissenschaftlichen Debatten eine wichtige Rolle (vgl. z. B. Berlit 2018a: S. 232f.; SVR 2018: S. 72) - und wird zu einem über das Migrationsrecht hinausweisenden Thema. Bereits Denninger geht im Hinblick auf die diffuse politische und rechtliche Zielbestimmung im Kontext von „Integration“ von der These aus, „dass dieser handlungstheoretisch wenig befriedigende Zustand nicht etwa Ausdruck der Gedankenoder Formulierungsschwäche der Verfasser der zahlreichen ,Integrations'Papiere ist, sondern seine Ursache im Problem der Integration selbst, in der (begrenzten) Fähigkeit der Menschen zur Vergesellschaftung und damit in der condition humaine überhaupt hat" (Denninger 2001: S. 446). Dafür nennt er verschiedene Gründe. Er weist darauf hin, dass „Integration" ein Grundproblem jeder Gesellschaft und jeden Staates ist und Integration das Verhältnis aller Staatsangehörigen oder „Einheimischen“ zum Staat und untereinander betrifft. Beleg dafür ist auch, dass Integration unabhängig von der Migrationspolitik in einem „umfassenden Sinne in der deutschen Staats- und Gesellschaftstheorie seit einem Jahrhundert ein zentrales Stichwort" ist (Gusy/Müller 2013: S. 269 mit weiteren Nachweisen). „Man sollte also nicht einmal als statistischen Indikator Erwartungshaltungen formulieren, die man für die Beziehungen zwischen ,Einheimischen nicht beachtet oder sich abgewöhnt hat" (Denninger 2001: S. 447). Geleitet von der Erkenntnis, „dass gesellschaftliche Integration, geschehe sie unter 
Inländern oder Ausländern oder von beiden untereinander, notwendigerweise ein inhaltlich unbestimmter und damit für politische Auseinandersetzungen offener Begriff ist und bleiben wird“ (ebd.: S. 448), kann „Integration" sinnvoll nur als ein ergebnisoffener Prozess beschrieben werden (vgl. ebd.: S. 447). Denn ,inhaltlich zielt das Integrationsziel auf Verständigung, ohne eine Gestaltform vorzugeben. Integration kann Verschiedenes bedeuten und eignet sich gerade wegen dieser Deutungsoffenheit als verfassungsrechtliche Orientierungsmarke, die im Sinne eines überlappenden Konsenses ein normatives Ziel vorgibt, zugleich jedoch verschiedene Ausgestaltungen zulässt" (Thym 2017: S. 198). Eine Konsequenz ist die kontinuierliche Aktualität als Gegenstand der Politik, eine „große Bandbreite möglicher Verdichtungsformen des sozialen ,Miteinander' oder ,Nebeneinander"“ und dabei „,die Kunst des Verbindens` zu üben und zu unterstützen“ (Denninger 2001: S. 448). „Integration“ kann damit nur als „ein komplexes, ganzheitliches Phänomen angemessen beschrieben und normativ begleitet werden. Integrationsvorgänge betreffen die ganze Gesellschaft und nicht nur den staatlichen Bereich; Vorgänge religiös-kultureller Integration und sozialer Integration dürfen nicht isoliert betrachtet, vielmehr müssen sie in Verbindung mit der politisch-rechtlichen Integration und ihren Wechselwirkungen aufeinander gesehen werden" (ebd.: S. 448f.).

Das „Phänomen Integration bezeichnet in unserem Kontext vornehmlich zwei Dimensionen: Ein Ziel und einen Prozess, der auf das Ziel gerichtet ist. Da aber auch das Ziel kein statisches ist, ist der Prozess auch niemals abgeschlossen, sondern ein tendenziell unendlicher, in welchem Intention und Handeln einander zudem wechselseitig beeinflussen können. Darüber hinaus ist der Integrationsbegriff handlungsoffen, er spart also die Instrumente der Zielerreichung aus" (Gusy/Müller 2013: S. 269f.). Zudem existiert nicht nur ein Ziel, sondern es gibt mehrere, die jeweils gesellschaftlich verhandelt werden. Deshalb ist es notwendig, einen Diskurs über die inhaltliche Ausrichtung dieser Prozesse zu führen und in diesem Kontext die rechtlichen Rahmenbedingungen genau zu reflektieren. Damit rücken generelle gesellschaftliche Werte in den Vordergrund. Identitätsentwicklung, Autonomie, eigenverantwortliches Handeln in modernen, multikulturell geprägten komplexen Gesellschaften, die Entwicklung von gesellschaftlich anerkannten Konfliktlösungsmöglichkeiten, Unterschiede zu akzeptieren und andere religiöse und weltanschauliche Orientierungen zu respektieren sind nur einige Elemente eines komplexen Integrationsprozesses, der zu der angestrebten umfassenden und gleichberechtigten ökonomischen, kulturellen, sozialen und gesellschaftlichen Teilhabe führen soll. Auch in der juristischen Diskussion geht es inzwischen um die 
Gestaltung von Teilhabe als komplexes Integrationsverständnis (vgl. Berlit 2018a: S. 232). Integration wird verstanden „als möglichst chancengleiche Teilhabe aller Personen an den zentralen Bereichen des gesellschaftlichen Lebens, u. a. Teilhabe an Bildung, Ausbildung, Arbeit und den Schutzund Fürsorgesystemen des Rechts- und Wohlfahrtsstaates sowie soziale und politische Partizipation" (ebd.). Berlit bezieht sich dabei explizit auf das Jahresgutachten 2010 des Sachverständigenrats deutscher Stiftungen für Integration und Migration, der diese Position aktuell in seinem Jahresgutachten 2018 nochmals sehr differenziert dargelegt hat (vgl. SVR 2018a: S. 70ff.). Zu Recht weist der Sachverständigenrat darauf hin, dass „es nicht die Integration der Zugewanderten in die Gesellschaft geben kann. Es geht immer um die individuelle Teilhabe, die zwischen den Einzelnen wie auch in den verschiedenen Teilbereichen des gesellschaftlichen Lebens unterschiedlich ausgeprägt sein kann. Die Gesellschaft, der Staat und die Zugewanderten müssen zusammen einen geeigneten Weg finden, der gleichberechtigte Teilhabe ermöglicht (vgl. ebd.: S. 72; auch Berlit 2018a: S. 232). Diese Zielsetzung kann über ein „disziplinübergreifendes Verständnis“ der Komplexität von Integrationsprozessen erreicht werden, die „nicht als $\mathrm{Zu}$ stand, sondern als langwieriger, wechselseitiger, ,dauerhafter und ergebnisoffener interaktiver Prozess der ökonomischen, sozialen, kulturellen und politischen Teilhabe von Menschen auf lokaler, regionaler, nationaler und transnationaler Ebene' zu sehen ist" (Berlit 2018a: S. 232f.).

Als Ergebnis kann festgehalten werden, dass der Begriff „Integration“ alltagssprachlich immer noch eine große Rolle spielt, seine Verwendung somit auch Resultat eines strategischen Kalküls sein kann. Denn er kann inhaltlich beliebig gefüllt werden und kann damit als „leerer Signifikant“ im Sinne der Diskurstheorie von Ernesto Laclau und Chantal Mouffe dienen (vgl. Laclau/Mouffe 2015, vgl. auch Abschnitt 3.2.4). Für analytische Zwecke ist er aus genau diesem Grund aber nicht geeignet. Es ist deshalb sinnvoll, einen „epistemologischen Bruch“ im Sinne Gaston Bachelards (Bachelard 1987) zu vollziehen und die Bezugsebene zu wechseln. Wenn „Integration“ einen Prozess bezeichnet, der alle Gesellschaftsmitglieder in allen Lebenssituationen einbezieht, wird er völlig unspezifisch. Er sollte für analytische Zwecke ersetzt werden durch den Begriff der „Teilhabe“, der alltagssprachlich zwar auch vorkommt, dort aber eher eine Randexistenz innehat. 
3.1.2 „Teilhabe“ als konzeptionelle Basis des Integrationsmanagements

Dies bedeutet aber, dass sich die Ziele des Integrationsmanagements weniger an dem diffusen Begriff „Integration“ als vielmehr am Begriff der „Teilhabe" orientieren sollten. ${ }^{10}$

Aus dieser Perspektive ist es notwendig, den Begriff „Teilhabe“ rechtsund sozialwissenschaftlich zu präzisieren (3.1.2.1) und danach soweit zu operationalisieren, dass er in konkrete Handlungskonzepte einmünden kann (3.1.2.2).

\subsubsection{Teilhabe als rechts- und sozialwissenschaftlicher Leitbegriff ${ }^{11}$}

Wie die bisherigen Ausführungen zeigen, ist der Begriff „Integration“ sowohl aus der Perspektive der Rechts- wie der Sozialwissenschaften unpräzise und verkürzt. Integration als komplexen Prozess zur Realisierung von gesellschaftlicher, kultureller und politischer Teilhabe zu verstehen, wie etliche Autorinnen und Autoren vorschlagen, bedeutet, das Zusammenspiel von Teilhabechancen und Teilhabekompetenzen ins Auge zu fassen. Damit verschiebt sich der begriffliche Fokus von „Integration“ $\mathrm{zu}$ „Teilhabe“. Letzterer Begriff hat folgende Vorteile:

- Er bezeichnet sowohl einen Zustand wie einen Prozess.

- Er kann dazu dienen, die Kernelemente dieses Prozesses differenziert zu betrachten (Teilhabechancen und Teilhabekompetenzen).

- Er zentriert nicht umgangssprachlich auf eine besondere Personengruppe (Zugewanderte), sondern stellt zentrale gesellschaftliche Aufgaben insgesamt ins Zentrum.

Rechtswissenschaftlich ist der Begriff „Teilhabe“ bisher überwiegend im Kontext sozialrechtlicher Fragestellungen in Verbindung zur verfassungsrechtlichen Aufgabe der Sicherstellung eines menschenwürdigen Daseins diskutiert worden (vgl. Reis/Siebenhaar 2015 mit weiteren Nachweisen). Die Darstellung der verfassungsrechtlichen Rahmenbedingungen wird zei-

10 Es mag terminologisch verwirrend erscheinen, dann von „Integrations-, und nicht von „Teilhabemanagement" zu sprechen, ist aber dem Umstand geschuldet, dass der Begriff „Teilhabemanagement“ bereits doppelt in Anspruch genommen ist - einmal im Kontext des Bundesteilhabegesetzes, zum anderen im Rahmen eines Landesprogramms in Nordrhein-Westfalen, das sich an eine eng gefasste Zielgruppe von Migrantinnen und Migranten richtet.

11 Dieser Abschnitt wurde von Benedikt Siebenhaar verfasst. 
gen, dass diese Diskussion auch im Bereich „Integration von Ausländerinnen und Ausländern" geführt wird und weiter zu entwickeln ist.

Sozialwissenschaftlich kann das Teilhabekonzept als Versuch verstanden werden, einen gegenüber dem Begriff „Armut“ „positiven normativen Gegenbegriff“ zu generieren, der es erlaubt, die Dimensionen eines menschenwürdigen Daseins konkret zu beschreiben: „Teilhabe als sozialstaatliches Leitkonzept bezeichnet dabei die Schwelle, deren Unterschreiten öffentliches Handeln und soziale Sicherheitsleistungen auslösen soll" (Bartelheimer 2007: S. 5). Das Verfassungsgebot der Menschenwürde und der freien Entfaltung der Persönlichkeit impliziert Teilhabeansprüche an den Sozialstaat und Teilhabe am gesellschaftlichen Leben. Das soziokulturelle Existenzminimum beinhaltet neben den materiellen Teilhabeansprüchen nämlich auch „ein Recht auf Teilhabe am gesellschaftlich üblichen Leben“ (Bundesregierung 2005, zitiert nach Bartelheimer 2007: S. 5).

"Teilhabe“ ist mehrdimensional; Bartelheimer bezieht sich auf Ergebnisse der Lebenslagenforschung, wenn er eine Konkretisierung vorschlägt: Teilhabe am System gesellschaftlicher Arbeitsteilung, Wohnen, Einbindung in soziale Nahbeziehungen, politische Partizipation (vgl. Bartelheimer 2007). Dabei beinhaltet der Begriff „Teilhabe“ aber zweierlei: er unterstellt aktive, handelnde Subjekte (Teilhabekompetenz, vgl. Abschnitt 3.1.2.2) und bezieht diese auf die gesellschaftlichen Teilbereiche, die relevant sind zur Realisierung der „Teilhabe am Leben in der Gemeinschaft“" (vgl. \$9 SGB I). Teilhabe berührt den verfassungsrechtlichen Rahmen und wird normativ „aufgeladen“, denn die beiden genannten Elemente stehen in engem Zusammenhang mit der Menschenwürde, bzw. einem Leben, das der Würde des Menschen entspricht: „Der verfassungsrechtliche Rahmen wird namentlich durch die Verfassungsziele des Sozialstaates und des Rechtsstaates, aber auch durch den tragenden Grundsatz der Menschenwürde und die Grundrechte bestimmt. Sie bilden Grund und Grenze sozialgestaltender Interventionen“ (Wallerath 2008: S. 159). Das Bundesverwaltungsgericht hat in einer sehr frühen Entscheidung den Zusammenhang von Sozialstaatsprinzip, Menschenwürde und Grundrechten bei der Umsetzung von Fürsorgeleistungen hergestellt. Das Gericht betonte „erstmals die - einfachgesetzlich geregelte - Pflicht zur Gewährung öffentlicher Fürsorge - heute: Sozialhilfe und Arbeitslosengeld II - subjektiv-rechtlich und brachte sie mit Art. 1 und 2, Art. 3, 20 und Art. 79 Abs. 3 GG in Verbindung. Die Menschenwürde verbiete es, den Menschen ,lediglich als Gegenstand staatlichen Handelns zu betrachten, soweit es um die Sicherung des notwendigen Lebensbedarfs (...), also seines Daseins überhaupt $^{`}$ gehe. Das folge , auch aus dem Grundrecht der freien Persönlichkeit 
(Art. 2 Abs. 1 GG) ' sowie dem Grundsatz des ,sozialen Rechtsstaates “ (Wallerath 2008: S. 159). Damit wird deutlich, dass unter „Teilhabe“ weitaus mehr zu verstehen ist als eine gesicherte materielle Existenz. Denn unter Menschenwürde ist im Grundgesetz der dem Menschen als solchem zustehende Eigenwert zu verstehen, der ihn zu eigenverantwortlichem Handeln befähigt. Die vom Sozialleistungsrecht zu gewährleistende Sicherung des menschenwürdigen Daseins umfasst auch die Schaffung und Aufrechterhaltung von Lebensumständen, die sowohl die Persönlichkeit des einzelnen Menschen wie dessen persönliche Handlungs- und Entfaltungsmöglichkeiten erhalten und fördern. Der Einzelne darf nicht zum „Objekt staatlichen Handelns werden und in seinem 'Selbstbestimmungsrecht' nicht in unverhältnismäßiger Weise beeinträchtigt werden" (Steinbach 2014: $\mathbb{1}$ SGB I, Rz 32). Somit manifestiert sich Menschenwürde gem. Art. 1 GG u. a. in der Schaffung eines möglichst weiten individuellen Autonomiebereichs, in dem sich die Potenziale jedes Menschen entfalten können. Dies hat bereits früh der ehemalige Bundesinnenminister Maihofer folgendermaßen zum Ausdruck gebracht: „Die Menschenwürde hat zwei Komponenten:

a) Die personelle: Die Bereitschaft und Fähigkeit des Menschen, mit sich in Einklang zu leben, also seine individualspezifischen Begabungen, Eigenheiten und Neigungen, die ihn als ein einmaliges Lebewesen kennzeichnen, in Auseinandersetzung und in Zusammenarbeit mit anderen Menschen zum Ausdruck zu bringen und selbstverantwortlich, im Sinne der Lebenserhaltung und Lebensbereicherung, zu entfalten.

b) Die gesellschaftliche: Die Bereitschaft und Fähigkeit der für den Menschen jeweils bedeutsamen gesellschaftlichen Umgebung, ihm dies nicht nur zu ermöglichen, sondern ihn dabei zu fördern" (Maihofer/ Behrend 1967: S. 543).

In Anbetracht dieser Komponenten muss staatliche Unterstützung auch Hilfe zur Selbsthilfe sein: "Jedenfalls greift in Ansehung der Würde jede staatliche Maßnahme zu kurz, die sich auf die finanzielle Absicherung des Existenzminimums beschränkt“ (Neumann 2010: S. 104).

Wichtig für den hier diskutierten Zusammenhang ist es, dass die grundlegenden rechtlichen Aussagen zur „Teilhabe“ auch für Zugewanderte gelten. Dabei muss die Prüfung der verfassungsrechtlichen Rahmenbedingungen zwischen der Bedeutung der Staatszielbestimmungen und der Grundrechte für Ausländerinnen und Ausländer unterscheiden. Aus den Staatszielbestimmungen oder sogenannten Staatsstrukturprinzipien wie dem Demokratieprinzip nach Art. 20 Abs. 1 GG und dem Rechtsstaatprinzip nach Art. 20 Abs. 3 GG lassen sich nur sehr eingeschränkt unterstützen- 
de Maßnahmen zur Herstellung der Teilhabe von Zugewanderten herleiten. „Zwar soll das Demokratieprinzip nicht auf staatliche, sondern auch auf gesellschaftliche Kontexte wie z. B. Schule, den Arbeitsplatz oder sozialstaatliche Institutionen Anwendung finden und auch Ausländern ein Mindestmaß an Teilhabe ermöglichen, beispielsweise durch ein Recht auf die Wahl von Betriebsräten oder Sozialversichertengemeinschaften. Doch die Möglichkeiten der Teilhabe sind bei Weitem nicht ausgeschöpft“ (Eichenhofer/Dilmaghani 2017: S. 6). So sind z. B. Drittstaatsangehörige im Gegensatz zu Unionsbürgern auf kommunaler Ebene bisher nicht wahlberechtigt. „Das Rechtsstaatsprinzip sichert ausländischen Staatsangehörigen zwar eine am Prinzip der Verhältnismäßigkeit orientierte Behandlung zu, doch folgen auch hieraus keine effektiven Teilhabechancen“ (ebd.). Die Beachtung des Verhältnismäßig-keitsgrundsatzes hat z. B. Bedeutung für die Regelungen zur Integration im Aufenthaltsgesetz. So kann die ausländerrechtliche Pflicht zur Teilnahme an den Integrationskursen unter dem Aspekt „Fördern und Fordern“ als ein Verstoß gegen den Verhältnismäßigkeitsgrundsatz gesehen werden (vgl. Eichenhofer 2013: S. 85). Dagegen enthält das Sozialstaatsprinzip gem. Art. 20 Abs. 1 und Art. 28 Abs. 1 GG „wichtige Vorgaben für die Regelung der Integration von Ausländern. Zwar lässt sich ihm kein Gebot entnehmen, bestimmte ausländeroder sozialrechtliche Maßnahmen zu ergreifen. Denn das Sozialstaatsprinzip gilt als abstraktes Staatsziel ohne nähere Qualifizierung durch formelle oder verfahrensmäßige Tatbestandsmerkmale" (ebd.: S. 83). Wesentliche Elemente des Sozialstaatsprinzips sind u. a. „ein menschenwürdiges Existenzminimum für jedermann" und die „Hebung des allgemeinen Wohlstands und die Ausbreitung der Teilhabe daran" (Zacher 1987, Rnr. 27,60). Das Grundgesetz enthält zwar „keinen detaillierten Katalog sozialer Grundrechte“ (Kötter 2016: S. 44), „begründet aus Art. 1 Abs. 1 GG in Verbindung mit dem Sozialstaatsprinzip des Art. 20 Abs. 1 GG umfasst das moderne Grundrecht auf Gewährleistung eines menschenwürdigen Existenzminimums aber (...) nicht nur die Sicherstellung der physischen Existenz des Menschen, sondern auch die Mittel für ein Mindestmaß an Teilhabe am gesellschaftlichen, kulturellen und politischen Leben" (Weth 2016: S. 15). In seiner Entscheidung zum Asylbewerberleistungsgesetz vom 18.7.2012 hat das Bundesverfassungsgericht den uneingeschränkten personellen Anwendungsbereich des Grundrechts auf Gewährleistung eines menschenwürdigen Daseins (vgl. Kötter 2016: S. 68) begründet: „Art. 1 Abs. 1 GG erklärt die Würde des Menschen für unantastbar und verpflichtet alle staatliche Gewalt, sie zu achten und zu schützen. (...) Als Menschenrecht steht dieses Grundrecht deutschen und ausländischen Staatsan- 
gehörigen, die sich in der Bundesrepublik Deutschland aufhalten, gleichermaßen zu“ (BVerfG, Urteil vom 18.7.2012 - 1 BvL 10/10, BvL 2/11, Rz 89). Für die Gestaltung der Integrationsförderung und Teilhabe von Ausländerinnen und Ausländern lassen sich daher „die folgenden verfassungsrechtlich garantierten Essentials der Menschenwürde festhalten: 'Das Grundrecht auf Achtung der Menschenwürde ist unverfügbar, nicht relativierbar, ohne Einschränkungsvorbehalt (anders als die anderen Grundrechte). Die Menschenwürde ist auch ,migrationspolitisch nicht zu relativieren (BVerfG, Urteil vom 18.7.2012 - 1 BvL 10/10, 1BvL 2/11, Rz 121). (...) Menschenwürde und Freiheitsrechte aus Art. 2 Abs. 1 GG garantieren dem Individuum Autonomie und selbstbestimmte Lebensführung. Schutz und Achtung der Menschenwürde geben nicht nur ein Recht auf Abwehr staatlicher Eingriffe, sondern auch ein Recht auf Teilhabe am gesellschaftlichen, kulturellen und politischen Leben" (Weth 2016: S. 15f.). Damit werden Autonomie, selbstbestimmte Lebensführung und Teilhabe als migrationspolitisch nicht zu relativierende Elemente des Grundrechts auf Achtung der Menschenwürde und wesentliche Elemente des Sozialstaatsprinzips zu zentralen Leitbildern für die Integrationsförderung von Ausländerinnen und Ausländern.

Zur Sicherstellung dieser verfassungsrechtlich gebotenen Unterstützung zur Teilhabe fehlen insbesondere für den Personenkreis der Zugewanderten, der keine Leistungen nach SGB II oder SGB XII beanspruchen kann, entsprechende einfachgesetzliche Regelungen zur Sicherstellung der dafür erforderlichen Hilfen und personenbezogenen Dienstleistungen. Insbesondere für Personen, die Leistungen nach dem Asylbewerberleistungsgesetz oder Analogleistungen nach SGB XII erhalten gibt es keine bundesgesetzlichen Vorgaben zur Erbringung entsprechender Dienst- oder Unterstützungsleistungen. Die Frage, ob diese Lücke innerhalb eines speziellen „Migrations- und Integrationsrechts" oder durch entsprechende Ergänzungen der allgemeinen gesetzlichen Regelungen zu schließen ist, soll hier nicht weiter vertieft werden. Wichtiger ist, den auf Grund der dargelegten verfassungsrechtlichen Vorgaben bestehenden Handlungsbedarf auf bundesund landesgesetzlicher Ebene politisch zu erkennen und per Gesetzgebung darauf zu reagieren.

\subsubsection{2 „Teilhabe“ aus der Perspektive des Capability Approach}

Die bisherigen Ausführungen haben gezeigt, dass „Teilhabe“ ein mehrdimensionaler relationaler Begriff ist, er bezieht sich auf objektive gesell- 
schaftliche Strukturen und Angebote und gleichzeitig auf deren subjektive Wahrnehmung (im doppelten Wortsinne). Deshalb gibt es zwei Ebenen der Realisierung von Teilhabe: Gesellschaftliche Bedingungen („gesellschaftliche Umwandlungsfaktoren" - Normen, Recht, Infrastruktur usw.; Teilhabechancen) (vgl. Bartelheimer 2007: S.4) sowie individuelle Fähigkeiten („individuelle Umwandlungsfaktoren“; Teilhabekompetenzen). Hieraus resultieren Verwirklichungschancen. Wie diese dann real wahrgenommen werden, wird durch die Anzahl und Qualität von Wahlmöglichkeiten auf der einen Seite und durch (individuelle wie gesellschaftliche) Zielsetzungen beeinflusst. Aus dieser tatsächlichen (und je nach Person, Region und Umfeld sehr unterschiedlichen) Wahrnehmung ergeben sich dann Integrations- oder Teilhabeergebnisse (Schaubild 1; vgl. ebd.: S. 9).

\section{Schaubild 1: Teilhabechancen und Teilhabekompetenzen}

Institutionelle Bedingungen

(z. B. Zuschnitt, Erreichbarkeit)

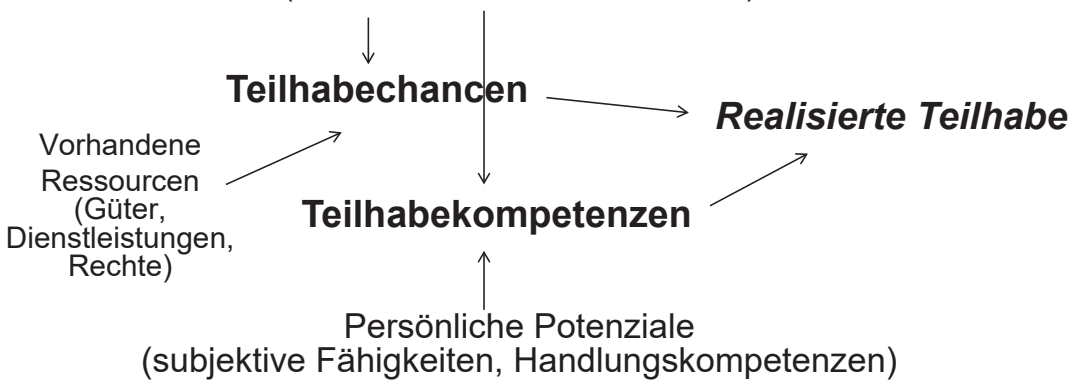

(Eigene Darstellung)

Die in beiden Begriffen angelegte Komplexität kann durch den Rekurs auf den "Capability Approach“ systematisiert und differenziert werden. Ähnlich wie der ältere Lebenslagenansatz (vgl. Weisser 1956; Leßmann 2007) will der „Capability Approach“ (in der deutschen Übersetzung Befähigungsansatz) den Wohlstand einer Gesellschaft mit Hilfe eines Maßes erfassen, das abbildet, über welche Befähigungen ein Mensch verfügen muss, damit dieser sein Leben erfolgreich gestalten kann? Mit anderen Worten: Was benötigt ein Mensch für ein gelungenes Leben bzw. für eine gelungene „Lebensführung“? Dieser Ansatz beinhaltet die Forderung an die Gesellschaft, „aktiv zur Entwicklung eines besseren Lebens aller Mitglieder (...) beizutragen" (Sen 2000: S. 52) und umfasst sowohl passive Freiheiten (als 
Abwesenheit von Hindernissen) und aktive Freiheiten (nach eigenen Wünschen handeln zu können). Instrumentell zählt Sen hierzu:

- Politische Freiheiten (Kritik, Widerspruch, Wahlrecht usw.).

- Ökonomische Institutionen (Ressourcen, Bedingungen des Tausches, Verteilung).

- Soziale Chancen (Bildung, Gesundheit).

- Transparenzgarantien (Pressefreiheit, Informationspflichten z. B. gegen Korruption).

- Soziale Sicherheit (Arbeitslosenversicherung, Sozialhilfe, Mindestlöhne).

Der Capability Approach fokussiert darauf, Ungleichheit in möglichst vielen Dimensionen zunächst zu erfassen (vgl. Bartelheimer 2009: S. 50), um dann Strategien zu ihrer Bearbeitung bzw. Überwindung abzuleiten. Zur Bewertung von Wohlfahrt sollen grundsätzlich sowohl die objektiven Möglichkeiten als auch die verfügbaren Verwirklichungschancen herangezogen werden, also die subjektive Fähigkeit, etwas zu erlangen sowie das objektive Vermögen, dies auch real zu erreichen - „nicht nur Fahrrad fahren können, sondern auch über ein Fahrrad verfügen zu können“ (Bonvin 2006, zitiert nach Bartelheimer 2007: S. 10) - und das Fahrrad gefahrlos nutzen zu können (z. B. durch das Vorhandensein von Fahrradwegen). Der Ansatz schlägt somit eine Brücke zwischen strukturellen Gegebenheiten und realer „Lebensführung“.

Der Ansatz ist (analog zu dem Begriff „Teilhabe“) multidimensional, indem er den Menschen in seinen gesamten Lebensverhältnissen und nicht einzelne Lebenslagen in den Fokus stellt, und dynamisch, da er auf das Verhältnis von objektiv gegebenen „Chancen“ und deren Verwirklichung (durch subjektives Handeln) abhebt. Zur Bewertung von Wohlfahrt werden deshalb die objektiven Möglichkeiten (Ressourcen), die verfügbaren Verwirklichungschancen und die Freiheit der Artikulation von Wünschen („Capabilities of voice“, vgl. Bonvin/Farvaque 2005) herangezogen. Letztlich geht es um den Raum an Möglichkeiten und Fähigkeiten, über Handlungsweisen (und damit Daseinsweisen) entscheiden zu können - Grundlage einer potenziell gelingenden Selbstverwirklichung - der zentralen Referenz von Menschenwürde. Gesellschaftlich vorhandene Ressourcen, seien es materielle Güter oder formale soziale Rechte, werden nur dann für die Individuen nutzbar, wenn einerseits die institutionellen Bedingungen gegeben sind (z. B. Rechte auch eingeklagt werden können), andererseits auch individuelle Kompetenzen und Fähigkeiten existieren, um die Ressourcen zu nutzen. Dabei ist es wiederum eine Folge institutioneller Bedingungen (z. B. des Bildungssystems), ob und wie diese Fähigkeiten ge- 
fördert werden. Sind sowohl die institutionellen Bedingungen als auch die persönlichen Potenziale gegeben, ist es eine Frage der Willensentscheidung, wie die tatsächlich realisierte Lebensweise aussieht. Hier existiert potenziell ein nicht aufhebbares Spannungsverhältnis zwischen gesellschaftlich gewährleisteten Verwirk-lichungschancen und deren individueller Nutzung. Deshalb geht der Verfahrensaspekt von Freiheit über die Frage hinaus, ob Teilhabechancen objektiv bestehen und Kompetenzen ausgebildet sind, sie zu realisieren. Vielmehr ist weitergehend danach zu fragen, welche Chance Personen haben, eigene Wünsche und Bedürfnisse zu artikulieren, die u. U. quer zu dem stehen, was „sozial erwünscht“ ist und den gesellschaftlich definierten „Verwirklichungschancen“ entspricht - nicht unbedingt jedoch den je individuellen Präferenzen: „Ein Fokus der Mitsprachemöglichkeit liegt in der aktiven Einbindung von Individuen in den Regelsetzungsprozess. Wenn alle betroffenen Individuen aktiv involviert sind im Regelsetzungsprozess (...), dann haben die Normen (...) einen gröBeren positiven Einfluss auf das Leben des Einzelnen (...). Der Befähigungsansatz (...) impliziert eine aufrichtige und für beide Seiten wohlwollende Reflexivität zwischen politischen und/oder sozialen Normen und individuellen Freiheiten“ (Bonvin/Farvaque 2005: S. 283f.). Deshalb gehört (politische) Partizipation zu den Grundelementen von Teilhabe.

Der Capability Approach setzt die Trennung von Teilhabechancen und -kompetenzen, strukturellen Bedingungen und Handeln voraus, um hieraus normative Forderungen abzuleiten. Es geht ihm nicht um eine theoretisch fundierte Verknüpfung von Teilhabechancen und -kompetenzen und er ist auch nur begrenzt für empirische Analysen dieser Vermittlung operationalisierbar. Auch die Felder, in denen analog zum Lebenslagenkonzept, Teilhabe „gemessen“ werden kann, sind eher alltagspraktisch bestimmt als theoretisch abgeleitet. Beide Einschränkungen legen nahe, die bisherigen Überlegungen durch eine Ausweitung des theoretischen Rahmens zu vertiefen.

\subsection{Teilhabe und Zuwanderung}

\subsubsection{Teilhabe und das Feld sozialer Hilfen}

„Teilhabe“ bedeutet, objektiv wie subjektiv an zentralen gesellschaftlichen Feldern partizipieren zu können; sowohl die (objektive) Chance hierzu zu haben wie auch die (subjektiven) Kompetenz(en), sich in diesen Feldern als kompetente Akteurin oder kompententer Akteur zu bewegen. Um die 
hiermit verbundenen objektiven wie subjektiven Herausforderungen aus einer sozialwissenschaftlichen Perspektive darzustellen, wird im Folgenden auf die Feldtheorie Pierre Bourdieus zurückgegriffen. Bourdieu geht davon aus, dass „Gesellschaft“ verstanden werden kann als fragiles Gebilde, das mehrere zentrale Felder zusammenfasst - diese werden als autonom, nach eigenen Regeln agierend konzipiert. Trotz dieser Autonomie gibt es natürlich Verbindungen zwischen den Feldern, die aber immer wieder ausgehandelt werden, d. h. nicht als gegeben vorausgesetzt werden können.

Im Folgenden wird der Begriff „Feld“ also nicht umgangssprachlich, sondern in enger Verbindung zur Feldtheorie des französischen Soziologen Pierre Bourdieu verwendet. Bourdieu formulierte seine Feldtheorie nur in Grenzen konsistent, sie stellt kein geschlossenes Theoriegebäude dar (vgl. Bourdieu/Wacquant 1996) und hat als Resultat unterschiedlichster empirisch gerichteter Arbeiten eine wechselvolle Geschichte durchlebt (vgl. ausführlich Bongaerts 2008). „Felder“ spielen in Bourdieus Gesellschaftstheorie eine wichtige Rolle dabei, den Zusammenhang zwischen (objektiv gegebener) „Struktur“ und (subjektiver) „Handlung“ begrifflich zu fassen. Bongaerts formuliert dies folgendermaßen: „Soziale Felder werden als relativ autonome Mikrokosmen konstruiert..., in denen eine feldspezifische Kapitalform zirkuliert, in denen spezialisierte und professionalisierte Akteure agieren und in denen nach bestimmten Regeln (nomos) bestimmte Interessen verfolgt werden, die auf einer affektiven Besetzung des Handlungsfeldes (illusio) eines Feldes ruhen. Die Konzeptionen von Kapital, nomos und illusio eines Feldes erlauben die Abgrenzung verschiedener Felder voneinander" (ebd.: S. 112, Hervorhebung im Original). Während mit „nomos“ die objektiv beschreibbaren Regeln und Wissensbestände eines Feldes bezeichnet werden, schlägt die „illusio“ die Brücke zum (subjektiven) Habitus, da hiermit „die affektive Bindung der Akteure an ein Feld und dessen spezifische Praxisformen begriffen wird. Illusio bezeichnet den unbedingten Glauben der Akteure an den Sinn und vor allem den Wert des Spiels, an dem sie beteiligt sind“ (ebd.: S. 114, Hervorhebung im Original). Dies einigt die Konkurrenten, die innerhalb eines Feldes um die Dominanz kämpfen, wie Emirbayer/Williams am Beispiel von New Yorker Hilfsorganisationen für Obdachlose zeigen: „The field of homeless shelters is a space riven with tensions and mutually opposing strategies of action. However, it is also worth noting that all of those involved in this field, regardless of the particular position they occupy within it, are heavily invested in the work of helping homeless people; homelessness is recognized across the board as a problem that is worth addressing. Their underlying 
belief in the game, or illusio, serves as the fundamental premise upon which the entire field is based, a shared understanding that goes without saying. The very willingness of the various actors in this field to engage in struggle over the mechanisms of service delivery, definition of a challenging client, reasonable lengths of stay, and whether or not shelters ought to maintain their right of refusal supports this shared framework of investments and commitments" (Emirbayer/Williams 2005: S. 705). Über diese „illusio" stellen sich die Akteure auf die objektiven Regeln des Feldes ein und entwickeln einen entsprechenden subjektiven Habitus, der es ihnen erlaubt, sich ohne beständige Reflexion im jeweiligen Feld kompetent zu bewegen. Auf diese Weise werden auch die „Kapitalformen“ inkorporiert. „Über den unbedingten Glauben an das Spiel hinaus umfasst der feldspezifische Habitus als eidos zudem die selbstverständlichen Weltsichten und Überzeugungen, die das Fundament der sozialen Praxis bilden. Diese Selbstverständlichkeiten sind die historischen a priori der sozialen Praxis (...). Kurzum: Sie bilden die feldspezifische doxa" (Bongaerts 2008: S. 123, Hervorhebung im Original). Diese „Doxa“ geht über rein kognitiv erfassbare Wissensbestände hinaus, sie umfasst vielmehr auch nicht-sprachliche Orientierungen und körperbezogene Ritualisierungen, „die körperliche Verankerung der in Form von Dispositionen inkorporierten sozialen Strukturen" (ebd.: S. 119).

In Bourdieus Ansatz spielt die Frage der Machtverhältnisse eine zentrale Rolle, dargestellt in der Verfügung über das jeweils feldspezifische Kapital. Dies gilt auf der Ebene der einzelnen Felder, erst recht aber auf der darüber liegenden Ebene des „Feldes der Macht“, das sich über die einzelnen Felder spannt: „Was in der sozialen Welt existiert, sind Relationen - nicht Interaktionen oder intersubjektive Beziehungen zwischen Akteuren, sondern objektive Relationen, die ,unabhängig vom Bewusstsein und Willen der Individuen' bestehen, wie Marx gesagt hat. Analytisch gesprochen wäre ein Feld als ein Netz oder eine Konfiguration von objektiven Relationen zwischen Positionen zu definieren. Diese Positionen sind in ihrer Existenz und auch in den Determinierungen, denen die auf ihnen befindlichen Akteure oder Institutionen unterliegen, objektiv definiert, und zwar durch ihre aktuelle und potentielle Situation (situs) in der Struktur der Distribution der verschiedenen Arten von Macht (oder Kapital), deren Besitz über den Zugang zu den in diesem Feld auf dem Spiel stehenden spezifischen Profiten entscheidet, und damit auch durch ihre objektiven Relationen zu anderen Positionen (herrschend, abhängig, homolog usw.)" (Bourdieu/ Wacquant 1996: S. 127). 
Im Zentrum der vorliegenden Überlegungen steht der Schnittbereich von Sozialpolitik und Migrationspolitik, somit „staatliche“ Aktivitäten. Der „Staat" ist nach Bourdieus Auffassung keine der Gesellschaft gegenüberstehende stillschweigend vorauszusetzende Einheit, sondern besteht einerseits aus einem gegliederten bürokratischen Feld mit spezifischen Machtverhältnissen, das aber gleichzeitig in andere Felder hineinreicht: „Das bürokratische Feld ist ein Feld, das alle Felder überspannt, ein Feld, in dem Eingriffe verfügt werden, die ökonomische sein können, wie etwa Subventionen, oder juridische, wie etwa die Einführung einer Rentengesetzgebung" (Bourdieu 2017: S. 638). Gleichzeitig ist der Staat aber auch Zurechnungspunkt der Formung von Wahrnehmungsweisen (als Bestandteil von Habitus), die ihn als „Einheit" konstituieren. Bourdieus Analyse verfolgt die Absicht: „die Autonomisierung eines bürokratischen Feldes zu beschreiben, innerhalb dessen die Staatsräson im objektiven und im subjektiven Sinne wirkt, das heißt eine bestimmte Logik, die nicht die der Moral, der Religion, der Politik usw. ist" (ebd.: S. 340). Das bürokratische Feld ist zergliedert in mehrere, inhaltlich abgrenzbare und beschreibbare Teilfelder mit spezifischen Kapitalien: „Der Ausdruck spezifisches Kapital besagt, dass dieses Kapital in Verbindung mit einem bestimmten Feld, also in den Grenzen dieses Feldes, einen Wert hat und nur unter bestimmten Bedingungen in eine andere Art Kapital konvertierbar ist“" (Bourdieu 1993: S. 108). Dominant sind dabei das juridische und das administrative Kapital, d. h. die Verfügung über "gültige" Ausdeutungen von Rechtsnormen einerseits sowie die Regeln zu ihrer Operationalisierung im bürokratischen Handeln andererseits (z. B. Ausübung von Ermessen). Unterschiedlich ist der Grad an Autonomie der Felder. Diese hängt davon ab, ob es im Feld gelingt, eigenständige Wertbeziehungen (manifestiert in Kapitalien) zu etablieren. Wichtig ist dabei die Zuschreibung von Kompetenz: „Zu einer Kompetenz gehört ein ,Ressort', ein ,Zuständigkeitsbereich'; sie impliziert also Grenzen und einen Kampf um Grenzen, und alle großen Kämpfe eines Machtfeldes sind Kompetenzkämpfe. Das Wort ,Kompetenz' (in seinen beiden Bedeutungen von ,Fachkenntnis' und ,Zuständigkeit') ist auBerordentlich wichtig, es ist ein juridischer und zugleich ein technischer Begriff: Kompetenz ist das Recht, eine technische Kompetenz innerhalb eines bestimmten Zuständigkeitsbereichs auszuüben“" (Bourdieu 2017: S. 357). D. h., verschiedene Kapitalien werden eingesetzt, um ein Feld zu autonomisieren und in diesem Feld Kompetenz in diesem doppelten Sinne zu erwerben. Diese Kompetenz stellt dann feldspezifisches Kapital dar. Im Feld sozialer Hilfen geht es darum, über Hilfe zu entscheiden, d. h. Fälle zu konstituieren (Sozialdimension) und Ressourcen einzusetzen (Sach- 
dimension). Dabei spielen Diskurse eine wichtige Rolle (vgl. z. B. ebd.: S. 492), in denen auch darüber verhandelt wird, welche Formen von Wissen (juristisches, administratives, psychologisches, soziologisches, sozialpädagogisches) legitim eingesetzt werden können, um „Wahrheit“ zu produzieren.

Soziale Teilhabe ist in vielen gesellschaftlichen Bereichen alles andere als selbstverständlich, nicht nur für „Ausländerinnen“ und „Ausländer“, sondern auch für deutsche Staatsangehörige. $\mathrm{Zu}$ allen Zeiten gab es deshalb Bestrebungen seitens des Staates (im weiteren Sinne), aber auch seitens gesellschaftlicher Organisationen, sowohl Teilhabechancen wie Teilhabekompetenzen von Individuen oder ganzer sozialer Gruppen durch spezielle Unterstützungsleistungen und -angebote zu verbessern oder zu sichern. Bereits seit dem europäischen Mittelalter bildete sich ein System „sozialer Hilfe“ aus, das im Zeitverlauf immer stärker Bestandteil staatlicher „Wohlfahrt" wurde. Deshalb spricht einiges dafür, diese „sozialen Hilfen“ als Sub-Feld(er) des bürokratischen Feldes zu konzipieren und dabei das Augenmerk auf die Kämpfe zwischen Berufsgruppen (Juristinnen und Juristen, Verwaltungsfachkräfte, Sozialarbeiterinnen und Sozialarbeiter) um die Deutungshoheit in den einschlägigen Diskursen zu legen. Grundlage für die Konstitution dieser Felder ist es, dass für Mitglieder einer Gesellschaft die Partizipation an zentralen Lebensbereichen von entscheidender Bedeutung ist: Arbeit/Wirtschaft, Bildung, Wohnen, Gesundheit bilden die Eckpfeiler sozialer Existenz, weisen aber jeweils spezifische Selektionsund Ausschlussregeln auf. Die Zugehörigkeit zur „Gesellschaft“ ist für niemanden per se gegeben, vielmehr beinhaltet der (partielle) Ausschluss aus diesen Lebensbereichen in der Regel eine prekäre soziale Existenz. Dies wurde historisch als „soziale Frage“ thematisiert und thematisierbar, sukzessive hat sich (quasi zur „Exklusionsvermeidung") ein staatlich organisiertes „Feld“ sozialer Hilfe ausgebildet, das als solches seit den 80er Jahren des letzten Jahrhunderts verschiedentlich, aber selten systematisch mit systemtheoretischem Begriffsinstrumentarium (also nicht feldtheoretisch) ausgelotet wurde - insofern müssen unterschiedliche theoretische Konzepte kompatibel gemacht werden. In dieser Tradition begreift Frank Hillebrandt (2002) „Hilfe“ als institutionalisierten Komplex, der aus Organisationen, Regeln und Praktiken besteht. Dieser Komplex greift eine grundlegende Problematik funktional differenzierter Gesellschaften auf: „Auch die moderne Gesellschaft ist nicht ohne Menschen denkbar" (Hillebrandt 2002: S. 241) - gleichzeitig greifen die Funktionssysteme der Gesellschaft nur selektiv auf deren Fähigkeiten zu, inkludieren nur selektiv, d. h.: „Die moderne Gesellschaft muss, allgemein gesprochen, dafür sorgen, dass den 
Funktionssystemen ständig geeignetes Personal zur Verfügung steht, ist jedoch nicht in der Lage, alle Individuen in gleichem Maße zu berücksichtigen" (ebd.: S. 242). Hier sieht Hillebrandt im Anschluss an Bommes/ Scherr die gesellschaftliche Funktion von „Hilfe“: „Die gesellschaftliche Funktion des Hilfesystems ist die allgemeine stellvertretende Inklusion derjenigen Exklusionsindividuen, die durch die anderen Funktionssysteme nicht mehr hinreichend personal inkludiert werden. Diejenigen, die für die Funktionssysteme an Relevanz für personale Inklusion verlieren, werden für das Hilfesystem relevant" (Bommes/Scherr 1996: S. 114). Hillebrandt betont: „Das Hilfesystem ist also primär auf die humanen Folgeprobleme der modernen Inklusionsverhältnisse bezogen, weil es über eine Intervention in den Lebenslauf der Exklusionsindividuen dort Daseinsnachsorge betreibt, wo die Daseinsvorsorge des Wirtschaftssystems nicht greift" (Hillebrandt 2002: S. 243). Allerdings hängt der Erfolg der „stellvertretenden Inklusion" davon ab, dass sie in tatsächliche Inklusion einmündet, sonst bleibt nur „Exklusionsverwaltung“: „Diese Funktion ist jedoch erst dann erfüllt, wenn das Hilfesystem die stellvertretende Inklusion, die es zur Daseinsnachsorge bewerkstelligt, in eine Inklusion überführen kann, die gerade nicht von ihm, sondern von den anderen Funktionssystemen der Gesellschaft geleistet werden muss. Die stellvertretende personale Inklusion, also die Verwaltung des Ausschlusses von den Inklusionsverhältnissen durch das Hilfesystem, geschieht daher, um allgemeine Inklusionsfähigkeit zu erzeugen bzw. wiederherzustellen“ (ebd.: S. 243f.).

Empirisch lässt sich für die gegenwärtige Gesellschaft ein gegliedertes Hilfesystem identifizieren, das sowohl materielle wie (sozial)pädagogische Hilfen umfasst und dessen gesellschaftliche Funktion darin besteht, „stellvertretende Inklusion" dadurch sicherzustellen, dass an den Teilhabechancen und den Teilhabekompetenzen der in prekäre Lebenslagen geratenen Gesellschaftsmitglieder gearbeitet wird. Damit zeigt sich, dass „Teilhabe“ zweierlei bedeutet: Die chancengleiche Teilhabe an gesellschaftlichen Feldern (Teilhabe erster Ordnung) und die Teilhabe an den Hilfen, die die Gesellschaft bzw. staatliche Instanzen bereitstellen, um diese Teilhabe zu sichern (Teilhabe zweiter Ordnung). Letztere müsste beinhalten, dass einerseits für alle Gesellschaftsmitglieder Teilhabechancen eröffnet und andererseits für diejenigen Teilhabekompetenzen gestärkt werden, die diese Chancen nicht ohne Unterstützung wahrnehmen können. Das (nunmehr wieder im Anschluss an Bourdieu verstandene) Feld sozialer Hilfe zur Sicherung von Teilhabe spaltet sich empirisch in Teilfelder mit je spezifischen Akteuren und Beziehungen, die allerdings vielfältige Überlappungen und Schnittstellen aufweisen (z. B. Jugendhilfe, Sozialhilfe, Grundsi- 
cherung für Arbeitsuchende). Das Gemeinsame dieser Teilfelder ist das Ziel, das erreicht werden soll, um das sich die Akteure bemühen: Realisierung von Teilhabe über die Verbesserung von Teilhabechancen und Teilhabekompetenzen. Die Feldakteure, überwiegend korporative Akteure, setzen ihre Ressourcen (Kapitalien) ein, um dieses Ziel zu erreichen und entwickeln hierzu unterschiedliche Strategien.

\section{Schaubild 2: Teilhabe und soziale Felder}

Teilhaberelevante
Feld sozialer Hilfe
gesellschaftliche Felder

SGB VIII
S13 SGB VIII SGB VIII
SGB II, SGB III
Wohngeldgesetz,
SGB II, SGB XII

(Eigene Darstellung)

Die Kapitalien, die zur Zielerreichung in diesem Feld bzw. den Teilfeldern eingesetzt werden, sind das bürokratische Kapital ${ }^{12}$ und das sozialpädagogische Kapital ${ }^{13}$ (eingesetzt von Akteuren im Kontext Sozialer Arbeit). Gleichzeitig zeigt sich die Verknüpfung mit anderen Kapitalsorten, die in diesem Feld eine Rolle spielen: juristisches Kapital, wissenschaftliches Ka-

12 Die Verfügung über im Feld positiv bewertete administrative Verfahren und gleichzeitig die Anerkennung für deren kompetente/professionelle Anwendung.

13 Die Verfügung über im Feld positiv bewertete sozialpädagogische/sozialarbeiterische Methoden und Techniken und gleichzeitig die Anerkennung für deren kompetente/professionelle Anwendung. 
pital, soziales Kapital, kulturelles Kapital und ökonomisches Kapital. All diese Kapitalsorten erhalten ihre spezifische Bedeutung aber nur, wenn sie mit den feldspezifischen Kapitalien verbunden werden. Zudem entfalten sie ihre Wirkung nur dann, wenn sie durch gesellschaftliche Anerkennung als symbolisches Kapital gelten, also rückgebunden sind an (z. T. auch feldübergreifend geführte) Diskurse.

Bedeutsam für die Analyse des Feldes sozialer Hilfe ist neben der horizontalen Differenzierung eine vertikale: In allen Feldern sozialer Hilfe lässt sich ein (Teil-)Feld der Formulierung politischer Programme (auf Bundesund Landesebene) vom Feld der lokalen Umsetzung unterscheiden. Bourdieu zeigt dies eindrucksvoll in seiner Analyse des Marktes der Eigenheime, der beeinflusst wird durch gesamtstaatliche Feldaktivitäten (Wohnungspolitik) und durch lokale Feldaktivitäten (vgl. Bourdieu 2006). In den lokalen Feldern treten andere Akteure auf als in den Feldern politischer Programmgestaltung und die „Programmebene“ wird durch die spezifischen Kräfte des lokalen Feldes transformiert („Ebene der Umsetzung“ - Eversberg 2014). Das lokale Feld ist auch die Ebene, auf der die Adressatinnen und Adressaten der Hilfe erreicht werden. Wie dies geschieht und was sich auf diesem spezifischen Feld abspielt, muss Gegenstand empirischer Untersuchungen sein.

\subsubsection{Das Feld der Zuwanderungspolitik - erste Annäherungen}

Die bisherige Argumentation unterstellt eine zwar funktional differenzierte, aber „geschlossene“ Gesellschaft, das Phänomen der Zuwanderung und der an der "Grenze"14 wirksam werdenden Selektionen von Teilhabechancen wurde noch nicht thematisiert. Hierzu ist eine Perspektivenerweiterung erforderlich, denn nicht nur die einzelnen Felder mit ihren Selektionsregeln entscheiden über Teilhabe, vorgängig entscheidet der Staat mit der Gewährung von Teilhaberechten grundsätzlich über Teilhabechancen - das zeigt der Diskurs über „Citizenship“ sehr deutlich (vgl. Hess/Lebuhn 2014 und Lebuhn 2013). Dessen Ausgangspunkt ist Marshalls Konzeption

14 Mit „Grenze“ ist hier nicht alleine die physische Staatsgrenze gemeint, sondern auch die über Aufenthaltsrechte definierte „innere Grenze.“ Und diese Grenze ist flexibel - Aufenthaltsrechte sind abgestuft und bestimmen die Möglichkeiten der einzelnen Funktionssysteme, Inklusion zu definieren, höchst unterschiedlich (und differieren dann weiter nach Teilsystemen, z. B. im System Bildung bzw. Wirtschaft). 
unterschiedlicher „Staatsbürgerrechte.“ Marshall „untersuchte (...) neben dem Recht auf politische Teilhabe - vor allem dem aktiven und dem passiven Wahlrecht - die historische Durchsetzung von zivilen und sozialen Rechten. Zudem unterschied Marshall systematisch zwischen den unterschiedlichen Dimensionen von Zugehörigkeiten einerseits und der gesellschaftlichen Teilhabe andererseits und argumentierte, dass die formale (Staats-)Bürgerschaft keinesfalls mit der Vergabe substanzieller Rechte in allen gesellschaftlichen Bereichen einhergehen muss. In diesem Sinne lässt sich auch nur dann von ,full citizenship' sprechen, wenn eine umfassende Teilhabe in allen gesellschaftlichen Bereichen gewährleistet ist" (Hess/ Lebuhn 2014: S. 14). Hier geht es um den Kampf um die Gewährung und Ausweitung von Teilhaberechten, letztlich um die Durchsetzung einer „sozialen Staatsbürgerschaft" (Balibar 2010), in der die Staatsbürger ein Recht auf umfassende Teilhabe in der national verfassten Gesellschaft haben. Balibar spricht von „intensiver Universalität“, die er definiert als „Gleichheit, die nicht nur formell ist, sondern einen genau konturierten materiellen Gehalt hat. Es handelt sich um ein Ensemble sozialer Rechte, die in die (Staats-)Bürgerschaft eingeschrieben sind und aus jedem Individuum einen (...) Quasi-Besitzer eines Teils des kollektiven Besitzes machen“ (ebd.: S. 27). Allerdings ist diese Universalität auf Staatsbürger begrenzt, stellt aus der Perspektive eines „extensiven Universalismus“ nur einen „Partikularismus" dar (ebd.). Aus anderer, systemtheoretischer Perspektive beschreibt dies Michael Bommes folgendermaßen: „Mit der Entstehung des modernen Nationalstaates kommen die Spezifik der politischen Inklusionsform ,nationale Staatsbürgerschaft ${ }^{\star}$ und ihr partikularer Universalismus als Limitierung der Leistungsberechtigten durch Kontrolle des Zugangs zum Staatsterritorium und als Beschränkung der Leistungsberechtigungen von Ausländern gegenüber dem Wohlfahrtsstaat zur Geltung“ (Bommes 1999: S. 175). Etienne Balibar spricht vom national-sozialen Staat, der eine „Äquivalenz zwischen (Staats-)Bürgerschaft und Nationalität“ zementiere (Balibar 2010: S. 26) ${ }^{15}$, aber durch internationale Integrationsprozesse einerseits, Zuwanderung andererseits in die Krise gerate. Bommes zeigt, wie der Staat durch die Ausdifferenzierung von Zugangsrechten versucht, diese Krise zu regulieren und die „Ungleichheitsschwelle“ flexibel zu gestal-

15 Balibar ist sich der Problematik dieses Begriffs durchaus bewusst. Er verwendet ihn „ohne den provokanten Klang des Ausdrucks zu fürchten, das heißt, ohne ihn als eine getarnte Variante des Nationalsozialismus zu verstehen, sondern im Gegenteil als eine Alternative zu der „Lösung, die der Letztere im Rahmen einer bestimmten Konjunktur darstellte“ (Balibar 2010: S. 25). 
ten: „Aussiedler“ werden (unter besonderen Regularien) mit deutscher Staatsbürgerschaft ausgestattet, „illegal“ Zugewanderte ausgeschlossen (Bommes 1999: S. 177). Dazwischen liegt ein Geflecht aufenthaltsrechtlicher Differenzierung, das die Grundproblematik verdeckt: den Widerspruch zwischen (menschenrechtlich fundierter) universalistischer Gleichheitsprogrammatik und ihrer national-partikularen Realisierung.

Diese Überlegungen erlauben es, die Überlegungen zum Begriff der „Teilhabe“ weiter zuzuspitzen. Oben war die Rede von grundgesetzlich verankerten „Teilhaberechten“, die realisiert werden über Teilhabechancen und Teilhabekompetenzen. Mit der Thematisierung der Problemstellungen im Kontext des "national-sozialen Staates“ wird deutlich, dass die Wahrnehmung von Teilhaberechten von einer vorgängig erteilten „Teilhabeberechtigung" abhängt. Ein Streifzug durch einfachgesetzliche Regelungen würde zeigen, dass es unterhalb der Ebene verfassungsrechtlich garantierter Menschenwürde durchaus differenzierte (sozial-)rechtliche Regelungen gibt, die Rechte auf Leistungen und Angebote mit dem Aufenthaltsstatus verknüpfen - mit in der Praxis teilweise gravierenden Folgen (vgl. Frings u.a. 2018). Damit wird die praktische wie auch die theoretische Bedeutung des Aufenthaltsrechts mehr als deutlich.

Somit existieren im Hinblick auf die Teilhabe zugewanderter Menschen zwei Spannungsfelder:

- Das Spannungsfeld zwischen der verfassungsrechtlich garantierten Teilhabe einerseits und der nur "differentiellen Inklusion" (Brett/Neilson 2013), die sich aus einfachgesetzlichen (z. B. ausländerrechtlichen) Regelungen ergibt.

- Der Differenz zwischen formalen Rechten und deren tatsächlicher Realisierung (substanzielle Rechte).

Dabei kommt der kommunalen Ebene eine wichtige Rolle zu: „Untersucht man die Abstufung und Fragmentierung von Rechten, so wird darüber hinaus deutlich, dass (...) gerade die Institutionen des, lokalen Staates' (Krätke/Schmoll 1987) eine zentrale Rolle bei der Stratifizierung der migrantischen Bevölkerung spielen und sich Strategien von Kontrolle und Ausgrenzung mit Politiken der Gewährung und der Teilhabe systematisch verschränken“ (Hess/Lebuhn 2014: S. 19). Außerdem findet die Inanspruchnahme von Rechten ebenso auf der kommunalen Ebene statt wie Unterstützungsleistungen zur Erhöhung der Teilhabekompetenzen überwiegend lokal organisiert werden (von Kommunen, Wohlfahrtsverbänden, aber auch von ehrenamtlich Tätigen). Grundsätzlich lässt sich feststellen, dass unabhängig von der Differenz zwischen formalen und substanziellen Rechten bereits hinsichtlich der formalen Rechte von „civic stratification“, 
d. h. von einer staatlich intendierten Abstufung von Rechten und einer damit einhergehenden Stratifizierung der Bevölkerung gesprochen werden kann. (vgl. ebd.: S. 18).

Ergänzt man das Modell der oben diskutierten gesellschaftlichen Felder um das Phänomen der Zuwanderung, ergibt sich folgendes Bild (Schaubild 3).

\section{Schaubild 3: Die feldtheoretische Konstellation „Einwanderung“}

\begin{tabular}{|c|c|c|}
\hline $\begin{array}{l}\text { Einwanderungs- } \\
\text { modus }\end{array}$ & Feld sozialer Hilfe & $\begin{array}{c}\text { Teilhaberelevante } \\
\text { gesellschaftliche Felder }\end{array}$ \\
\hline (vereinfacht) & SGB VIII & $\begin{array}{l}\text { Frühkindliche } \\
\text { Bildung }\end{array}$ \\
\hline Mit Visum & $\S 13$ SGB VIII & $\begin{array}{l}\text { Schulische } \\
\text { Bildung }\end{array}$ \\
\hline $\begin{array}{l}\text { Ohne Visum } \\
\text { (unerlaubte }\end{array}$ & $\begin{array}{l}\S 13 \text { SGB VIII } \\
\text { SGB II, SGB III }\end{array}$ & Ausbildung \\
\hline $\begin{array}{l}\text { nach § } 14 \\
\text { AufenthaltsG) }\end{array}$ & SGB V, SGB XII & Gesundheit \\
\hline $\begin{array}{l}\text { Visafrei } \\
\text { (EU-Freizügigkeits }\end{array}$ & SGB II, SGB III & Arbeit \\
\hline $\begin{array}{l}\text { Migrations- } \\
\text { regime }\end{array}$ & $\begin{array}{l}\text { Wohngeldgesetz, } \\
\text { SGB II, SGB XII }\end{array}$ & Wohnen \\
\hline
\end{tabular}

(Eigene Darstellung)

Analog zum Feld sozialer Hilfen lässt sich ein „Feld der Zuwanderungspolitik" identifizieren, das ähnlich strukturiert ist wie das Feld sozialer Hilfe. ${ }^{16}$ Wir finden hier bereichsspezifische Gesetze und Organisationen (BAMF, ABH, Migrationsberatung), eigene Selektionsregeln, Akteure mit feldspezifischem Habitus (vgl. Eule 2014) etc. In der Migrationsforschung wird seit Längerem der Begriff des „Migrationsregimes“ gebraucht, der im Zentrum aktueller Debatten steht. Oltmer fasst „Migrationsregime“ fol-

16 Hier wird explizit von „Zuwanderung“ und nicht von „Migration“ gesprochen, um zu verdeutlichen, dass die Betrachtung auf das Verhältnis von Zuwanderung einerseits und nationalstaatlich geprägten sozialen Hilfen andererseits fokussiert. (vgl. auch Janotta 2018). 
gendermaßen: „Migrationsregime sollen hier verstanden werden als integrierte Gestaltungs- und Handlungsfelder institutioneller Akteure, die einen bestimmten Ausschnitt des Migrationsgeschehens fokussieren, Migrationsbewegungen kanalisieren und die (potenziellen) Migrantinnen und Migranten kategorisieren. Jedes Migrationsregime hat eigene institutionelle Akteure und spezifische migratorische Objekte, problematisiert, plant und handelt anders als andere Migrationsregime, umfasst mithin spezifische Regeln und Verfahren, Bedingungen und Formen des Sammelns von Informationen über einen migratorischen Sachverhalt, bewertet diese Informationen anders und vermittelt die Ergebnisse je verschieden in und zwischen institutionellen Akteuren, gegenüber den (potenziellen) Migranten und der Öffentlichkeit" (Oltmer 2018b: S. 247). Und: „Institutionelle Akteure können staatliche legislative, exekutive, judikative), suprastaatliche sowie internationale Instanzen sein oder kommunale Apparate, aber auch private Träger (Unternehmen, Vereine, Verbände). Ihre Interessen, Beobachtungsweisen, Normen und Praktiken bringen sehr unterschiedliche Kategorisierungen von Migrantinnen und Migranten hervor, die die gesellschaftliche, ökonomische, politische oder kulturelle Teilhabe am Zielort beeinflussen" (ebd.).

Das Feld der Zuwanderungspolitik kann mehr als ein „Migrationsregime" umfassen ${ }^{17}$; in der Realität zeigen sich konkurrierende „Regime“, die von unterschiedlichen Diskursen und institutionellen Arrangements geprägt sind Oltmer betont außerdem, dass Zuwanderung als Resultat eines Aushandlungsprozesses verstanden werden muss: „Beobachten lassen sich unterschiedliche Reichweiten und Wirkungsgrade im Wechselverhältnis von einerseits Normen, Strategien und Maßnahmen institutioneller Akteure des Migrationsregimes und andererseits Taktiken, Aktivitäten und Handlungen (potenzieller) Migranten. Auf diese Weise prägen, formen, (ko-)produzieren institutionelle und individuelle Akteure in Konflikt und Kooperation Migration. Nimmt man eine solche Perspektive ernst, kann es gelingen, Relationen, Hierarchien und Wechselverhältnisse offenzulegen, also das Handeln Einzelner oder Mikrostrukturen in Meso- und Makrokontexte bzw. -strukturen zu fügen“ (Oltmer 2018a: S.9). Insbesondere gilt es zu beachten, dass der Aushandlungsprozess auf die Verfasstheit der Akteure zurückwirkt, diese und ggf. ihre Strategien verändert. Ein „Migra-

17 Abgesehen von der Unterscheidung zwischen Mobilitäts- und Präsenzregime können auf Länderebene wie auf kommunaler Ebene unterschiedliche Migrationsregimes unterschieden werden. Dies führt u. U. dann zu politischen Verwerfungen zwischen Bund und Ländern etc. 
tionsregime" ist somit ein spezifischer institutioneller Komplex innerhalb des Feldes der Zuwanderungspolitik, der von spezifischen Diskursformationen geprägt ist und diese umgekehrt hervorbringt (vgl. Abschnitt 3.2.3). Dieser Komplex ist nicht konsistent, sondern besteht aus Elementen, die zueinander in Widerspruch treten können. Dies wird begrifflich gefasst über die Unterscheidung zwischen „Mobilitätsregime“ und „Präsenzregime“: „Migrationsregime verfügen immer über zwei elementare und miteinander verflochtene Felder: Erstens ,Mobilitätsregime', die auf die Einflussnahme auf den Zugang zu bzw. die Abwanderung aus einem Raum bzw. von einem Territorium verweisen, sowie zweitens ,Präsenzregime‘, die die Normen und Praktiken der Einbeziehung bzw. des Ausschlusses von Zugewanderten in gesellschaftlichen Funktionsbereichen wie beispielsweise Politik, Recht, Wirtschaft oder Erziehung umfassen. Präsenzregime rahmen mithin Integration, die als das permanente Aushandeln von Chancen der ökonomischen, politischen, religiösen oder rechtlichen Teilhabe verstanden werden kann" (Oltmer 2018b: S. 247). Die beiden Regimetypen können einander ergänzen oder konkurrieren. Ihre interne Konsistenz erhalten sie über spezifische Diskursformationen (s. u.). Beide bilden aber Bestandteile eines übergreifenden „Migrationsregimes“, das seinen spezifischen Charakter durch das Verhältnis der beiden Regimetypen zueinander erhält.

Greift man diese Überlegungen auf und liest sie feldtheoretisch gegen den Strich, kann man für das Feld der Zuwanderungspolitik ein zentrales „Enjeu“ im Sinne Bourdieus ${ }^{18}$ identifizieren: die Kompetenz, „Teilhabeberechtigungen" zu erteilen. Sie bildet sowohl einigendes Band als auch Trennlinie zwischen Mobilitäts- und Präsenzregime und ist gleichzeitig Ziel wie umkämpftes Objekt im Feld, um das unterschiedliche korporative Akteure ringen. Hier lassen sich als Pole identifizieren: mobilitätsorientierte Akteure/Strategien, die auf die Verweigerung bzw. restriktive Gewäh-

18 Im Deutschen häufig - etwas schief - mit „Einsatz“ in einem Spiel übersetzt; Bourdieu geht es aber sowohl um den Einsatz wie um den potenziellen Gewinn in einem Spiel: „In der Tat lässt sich das Feld mit einem Spiel vergleichen (...). So gibt es Einsätze bei diesem Spiel, (...) die im Wesentlichen das Produkt der Konkurrenz der Spieler untereinander sind; eine Investition in das Spiel, eine Besetzung (im psychologischen Sinn) des Spiels (...): Die Spieler sind im Spiel befangen, sie spielen, wie brutal auch immer, nur deshalb gegeneinander, weil sie alle den Glauben (...) an das Spiel und den entsprechenden Einsatz, die nicht weiter zu hinterfragende Anerkennung teilen (...), und dieses heimliche Einverständnis ist der Ursprung ihrer Konkurrenz und ihrer Konflikte" (Bourdieu/Wacquant 1996: S. 127ff.). 
rung von Teilhabeberechtigungen setzen, und präsenzorientierte Akteure/ Strategien, die im Gegensatz hierzu Teilhabeberechtigungen forciert gewähren möchten. Beide Akteursgruppen setzen die Kapitalien ein, über die sie verfügen: juristisches Kapital, administratives Kapital, soziales Kapital, kulturelles Kapital und ökonomisches Kapital, um das feldspezifische Kapital zu gewinnen bzw. zu erhöhen: die Kompetenz, über Teilhabeberechtigungen zu entscheiden bzw. Teilhabeberechtigungen zu erleichtern oder zu erschweren. Auch dieses feldspezifische Kapital ist verknüpft mit symbolischem Kapital, was sich in den Auseinandersetzungen um Zuwanderungspolitik in den letzten Jahren am Beispiel von Abschiebungen sehr deutlich zeigen lässt: Je nachdem, ob eine offensive Abschiebepraxis in der Öffentlichkeit positiv oder negativ konnotiert ist, erhöht oder vermindert sich das Ansehen derer, die hier besondere „Härte“ zeigen.

\subsubsection{Diskursformationen im Feld der Zuwanderungspolitik}

An dieser Stelle ist es sinnvoll, systematisch auf den Stellenwert gesellschaftlicher bzw. fachspezifischer Diskurse bzw. Diskursformationen einzugehen.

Bourdieu weist zwar immer wieder darauf hin, dass Positionen in Feldern über Diskurse eingenommen bzw. verteidigt werden, arbeitet aber kein konsistentes Konzept des „Diskurses“ aus. Gleichwohl haben mehrere Autorinnen und Autoren für die empirische Arbeit mit der Feldtheorie auf Diskursanalysen zurückgegriffen und sich dabei meist auf die Überlegungen Michel Foucaults gestützt. Dieser Vorgehensweise wird auch hier gefolgt, allerdings mit einer Akzentverschiebung in Richtung auf die Diskurstheorie von Ernesto Laclau und Chantal Mouffe. In diesem Sinne sind „Diskurse (...) strukturierte, partiell geschlossene Formationen, die aus artikulatorischen Praktiken hervorgehen. Die frei flottierenden Elemente werden bearbeitet und zu einer diskursiven Formation gebracht und in Beziehung gesetzt. Durch die artikulatorische Praxis transformieren sich die Elemente, so dass neue diskursive Formationen und Sinnzusammenhänge entstehen. Die Elemente bzw. die Signifikanten werden neu gebündelt und zu einem Knotenpunkt vernäht" (Moebius 2018: S. 133). Ein Diskurs besteht somit aus mehreren Sinnelementen, die - obwohl different (Differenzketten) miteinander verknüpft werden und damit einen Sinnzusammenhang bilden (Äquivalenzketten). Laclau/Mouffe fassen den Begriff des Diskurses bewusst weit und zielen darauf ab, die Unterscheidung zwischen diskursiven und nicht-diskursiven Praktiken zu unterlaufen. Somit kön- 
nen alle sozial-kulturellen Praktiken als „bedeutsame Praktiken“ als Bestandteile von Diskursen begriffen werden. „Der Diskurs ist hier keine spezifische ,Redeweise', sondern bezeichnet den relationalen Sinnzusammenhang, der sich in mündlicher Kommunikation oder schriftlichen Texten genauso manifestiert wie in Praktiken des Umgangs mit Objekten - etwa der Industrie - oder in ,schweigsamen' institutionellen Komplexen" (Reckwitz 2006: S. 341). Was Laclau und Mouffe als „Diskurs“ bezeichnen, als System von Äußerungen (Artikulationen) erscheint in Foucaults Terminologie als „diskursive Formation“: „In dem Fall, wo man in einer bestimmten Zahl von Aussagen ein ähnliches System der Streuung beschreiben könnte, in dem Fall, in dem man bei den Objekten, den Typen der Äußerung, den Begriffen, den thematischen Entscheidungen eine Regelmäßigkeit (eine Ordnung, Korrelationen, Positionen und Abläufe, Transformationen) definieren könnte, wird man übereinstimmend sagen, dass man es mit einer diskursiven Formation zu tun hat (...)“ (Foucault 1986: S. 58). Diskursive Formationen verknüpfen einzelne Diskurselemente und Teildiskurse zu einem kohärenten System. „Dabei bleiben sie jedoch nicht auf die - linguistische - Verknüpfung von Text(teilen) beschränkt. Neben der Begriffsformation regeln sie auch die Objektformation (Wie/In welcher Konstellation werden Objekte konstituiert), Subjektformation (Welchen Weisen der Produktion von Äußerungen müssen sich Sprechende unterwerfen) sowie die Denkperspektiven bzw. Strategien“ (Huke 2010: S. 12). Diskursive Formationen können miteinander konkurrieren, verschiedentlich lassen sich diskursive hegemoniale Projekte voneinander unterscheiden. Diskursphänomene geraten damit ,als strategische Spiele aus Handlungen und Reaktionen, Fragen und Antworten, Beherrschungsversuchen und Ausweichmanövern, das heißt als Kampf (Reisigl 2006: S. 101) in den Blick“" (Huke 2010: S. 23).

Im Hinblick auf das Feld der Zuwanderungspolitik können drei diskursive Formationen unterschieden werden, die zwar historisch chronologisch auftreten, sich aber gleichwohl überlappen. Diese Diskursformationen prägen die jeweiligen Mobilitäts- bzw. Präsenzregimes bzw. deren Verhältnis zueinander.

Die erste Diskursformation ist weitgehend geprägt von der Regulation des "Zugangs“ und Zugang wird als Frage der inneren Sicherheit verhandelt: "Zugang als Frage innerer Sicherheit basiert im Wesentlichen auf einer dichotomen Territorialisierung von Sicherheit und Unsicherheit als durch Grenzen getrennten Räumen und ist damit eng an das Konzept des Nationalstaats gebunden (...). Das ,Außen` wird als Problem für die Sicherheit des ,Innen‘ konzeptionalisiert, woraus die Notwendigkeit gefol- 
gert wird, einerseits die Grenzen zum ,Außen` zu sichern, andererseits die Präsenz des ,Außen` im ,Innen' möglichst restriktiv zu handhaben und zu kontrollieren (...). Zentrale Begriffe innerhalb der diskursiven Formation sind unter anderem Legalität/Illegalität, Kriminalität, Konflikte und Sicherheit“ (ebd.: S. 56). Historisch gründet dieser Diskurs im „Gastarbeitersystem“ (Oltmer/Kreienbrik/Sanz Diaz 2012) und dessen Engführungen des Migrationsthemas. „Die erste Phase der deutschen Integrationspolitik kannte nur eine individuelle bzw. gesellschaftliche Verantwortung für die Integration von Migrantinnen und Migranten. Im Ausländerrecht kam der Begriff nicht vor und auch in der Sache wurde die Förderung der Eingliederung in die deutsche Gesellschaft nicht als staatliche Aufgabe betrachtet. Dahinter stand die Leitvorstellung, die meisten Ausländer seien nur vorübergehend in Deutschland" (Groß 2007: S. 316). Das System der Anwerbeabkommen zwischen Aufnahme- und Entsenderstaaten basierte auf der Vorstellung eines nur temporären Aufenthaltes der „Gastarbeiter“. Insofern war der Aufenthalt eine Angelegenheit juristisch-administrativer Regulation und „Integration“ kein Thema. "Zur Zeit des ,Anwerbestopps" von 1973 (...) gab es angesichts der faktisch einsetzenden dauerhaften Niederlassung der Arbeitsmigranten und ihrer Familien keine klar formulierten migrations- und integrationspolitischen Rahmensetzungen des Bundes und der Länder. Daraus resultierte eine große kommunalpolitische und administrative Unsicherheit darüber, welche politischen Probleme auf die Kommunen zukommen würden“ (Bommes 2018: S. 107). Die mit der Arbeitsmigration verbundenen sozialen Probleme wurden an die Wohlfahrtsverbände delegiert („Ausländersozialarbeit“, finanziert durch die Bundesanstalt für Arbeit und damit die Versichertengemeinschaft, nicht über Steuermittel) bzw. auf die kommunale Ebene abgeschoben. Aus dieser Zeit resultieren unterschiedliche „Pfade“ der Entwicklung kommunaler Integrationspolitik. Diese hat - so Bommes - „gebastelten und heterogenen Charakter.“ (ebd.) In dieser Zeit dominiert eindeutig das „Mobilitätsregime“, das in der institutionellen Ausformung auf die Zeit vor dem 2. Weltkrieg verweist (Ausländerbehörden).

Die zweite Diskursformation manifestiert sich im Begriff der „Integration“ im Aufenthaltsgesetz von 2005. Dieses Gesetz „verwandelt die Integrationsförderung in eine Staatsaufgabe" (Groß 2007: S. 317). Wie in Abschnitt 3.1 gezeigt wurde, wird der Begriff „Integration“ jedoch nicht präzise definiert, sondern der Begriffsinhalt changiert. Gleichwohl erfüllt er eine klare Funktion: „Mittels der Verwendung (des Namens „Integration“, d. A.) grenzen sich die Sprechenden in den Integrationsdebatten nach drei Seiten hin ab: Erstens von „Assimilation“, die mit Zwang, Illiberalität und 
unrühmlichen Vergangenheiten assoziiert wird. Zweitens von der Vorstellung, auf Einwanderung ganz verzichten zu können. Diese in der bundesrepublikanischen Debatte noch in jüngerer Vergangenheit verbreitete Position wird als gestrig und unvernünftig bewertet. Und drittens vom „Multikulturalismus“, der - einerseits - mit Beliebigkeit, Werteverfall und Gefahr assoziiert und als naiv und grob fahrlässig bewertet wird. Andererseits gilt Multikulturalismus weiterhin als erstrebenswertes Ziel oder wird zumindest als gesellschaftliche Realität gerahmt. Die Notwendigkeit, Multikulturalität durch „gemeinsame Werte“, „Zielvorgaben“ und „Spielregeln“, „also mithilfe von Integrationsmaßnahmen auszubalancieren und zu zähmen, bleibt unbestritten“ (Buck 2011: S. 47). Das Aufenthaltsgesetz reguliert spezifische Leistungen für Zugewanderte (Integrationskurse, Beratung), verknüpft sie aber mit aufenthaltsrechtlichen Status, deren Definition den Kern des Aufenthaltsgesetzes bilden. Es behandelt somit den $\mathrm{Zu}-$ gang als humanitäre Frage: „Zugang als humanitäre Frage verknüpft in der Figur des Flüchtlings die Legitimität von Zugang mit dem Nachweis einer (individuellen) Verfolgung: Ist die Sicherheit eines Individuums gefährdet, wird ihm die Möglichkeit zugestanden, Zugang zum Territorium zu erhalten und Asyl zu beantragen. Welche Fluchtgründe als, legitim' und als Basis des Status ,Flüchtling' gelten, ist dabei immer abhängig vom historischen Kontext (...). Zentrale Begriffe der diskursiven Formation sind etwa Flucht/Flüchtling, Verfolgung, Schutz oder Menschenrechtsverletzung. Als Objekte werden unter anderem Flüchtlinge und Behörden konstituiert und zueinander in Beziehung gesetzt" (Huke 2010: S. 57). Hier spielt das Mobilitätsregime noch eine zentrale Rolle, Elemente des Präsenzregimes finden sich in der Form von „Spezialangeboten“ (Integrationskurse, Migrationsberatung). In der Folgezeit verschieben sich jedoch die Akzente: „Mit seinem Ansatz, die Integrationsförderung ( $\mathbb{S} 43 \mathrm{fff}$. AufenthG) nicht mehr vom Vorliegen eines rechtmäßigen Aufenthaltes bzw. eines bestimmten Aufenthaltstitel, sondern von einer, guten Bleibeperspektive' abhängig zu machen, trifft der Gesetzgeber eine pragmatische Entscheidung, die das Ziel der Integrationsförderung ( $\$ 1$ I 4 , $\$ \mathbb{S} 43 \mathrm{ff}$. AufenthG) in gewisser Weise über das der Zuwanderungssteuerung ( $\mathbb{1} 1$ I 1 AufenthG) stellt. Denn in die Integrationsförderung können nunmehr auch Ausländer einbezogen werden, denen noch keine Aufenthaltserlaubnis erteilt wurde" (Eichenhofer 2016: S. 257f.). Gleichzeitig mit der Ausweitung des potenziellen Adressatenkreises für integrative Maßnahmen wird aber die „Bringschuld“ der Zugewanderten hervorgehoben: „In die Grundsatznorm des $\$ 43$ I Aufenthaltsgesetz wird ausdrücklich das Fordern der Integration eingefügt, da die Ausländer, die dauerhaft in Deutschland bleiben wollen, ei- 
gene Anstrengungen zu ihrer Integration leisten müssen" (Groß 2007: S. 313). Groß stellt dazu fest: „Seit das deutsche Migrationsrecht das Thema Integration entdeckt hat, ist ein Trend zur Vorverlagerung und Verschärfung der Anforderungen festzustellen“ (ebd.: S. 319). Man könnte hier davon sprechen, dass Mobilitäts- und Präsenzregime in eine prekäre Balance gebracht werden, eine „Teilhabeberechtigung“ wird selektiv gewährt, die Selektion administrativ über die Definition von „Bleibeperspektiven" gesteuert. Verwaltungsstrukturell spiegelt sich dieser Diskurs in einer Reihe von „Sonderdiensten“ oder administrativen „Sondereinheiten", die sich speziell an (Gruppen von) Zugewanderte(n) richten ohne systematisch mit den „Regelstrukturen“ verbunden zu sein (Integrationsministerien, Integrationsämter, Beratungsdienste).

Die dritte Diskursformation kreist zwar auch um den Begriff „Integration" verbindet ihn aber mit anderen Inhalten. Hier sei zur Erinnerung wieder Berlit zitiert: „Integration als Herstellung von sozialer, gesellschaftlicher und politischer Einheit aus Vielfalt ist aber nicht auf die Bewältigung von Migrationsprozessen zu beschränken und fordert selbst in seiner thematischen Verengung neben den MigrantInnen zudem auch die Aufnahmegesellschaft, die durch Integrationsprozesse verändert wird. Ich folge dem Sachverständigenrat deutscher Stiftungen für Integration und Migration (SVR) und verstehe hier Integration als möglichst chancengleiche Teilhabe aller Personen an den zentralen Bereichen des gesellschaftlichen Lebens, u. a. Teilhabe an Bildung, Ausbildung, Arbeit und den Schutzund Fürsorgesystemen des Rechts und Wohlfahrtsstaates sowie soziale und politische Partizipation“ (Berlit 2018a: S. 231). Die enge Verknüpfung von „Integrationsförderung“ mit aufenthaltsrechtlichen Status wird gelockert, allerdings weniger verknüpft mit juristischen als mit ökonomischen Erwägungen. Somit wird Zugang als ökonomische Frage gefasst: „Zugang als ökonomische Frage verbindet Zugang mit ökonomischen ,Bedürfnissen'. Grenzüberschreitende werden als ökonomisch ,nützlich ${ }^{\text {/nicht , nützlich }}{ }^{\circ}$ und damit als Arbeitskräfte angerufen und konstituiert. Zentrale Begriffe, die zueinander in Beziehung gesetzt werden, sind etwa Arbeitsmarkt oder Arbeitslosigkeit, demographischer Wandel, wirtschaftliche Entwicklung, Angebot und Nachfrage nach Arbeitskräften oder Qualifikation" (Huke 2010: S. 57f., Hervorhebung im Original). Im Vordergrund steht „Teilhabe" und es ist klar, dass diese nicht mit „Sondermaßnahmen" realisiert werden kann. So zieht Berlit auch klare konzeptionelle Konsequenzen aus seinem teilhabeorientierten Integrationsbegriff: „Integration als Querschnittsaufgabe, die (nahezu) alle Verwaltungsbereiche erfasst, ist auch einer der zentralen Gründe gegen eine spezialisierte, konzentrierte ,Inte- 
grationsverwaltung' mit umfassender, auch operativer Zuständigkeit. Dies führte zu vermeidbaren Reibungsverlusten. Effektiver sind die kulturelle Öffnung aller Bereiche der Verwaltung und die konzeptionelle und personelle Vernetzung der in den jeweiligen Fachverwaltungen tätigen ,Integrationsfachleute', welche die spezialisierte Fachkompetenz mit übergreifenden Integrationsanforderungen verbindet“ (Berlit 2018b: S. 289). Berlit argumentiert weiter: „für eine dauerhafte Optimierung des Migrations- und Integrationsmanagements in Regelsituationen bedarf es lediglich entsprechender Koordinations- und Kooperationsstrukturen, um die kontinuierliche strategische und operative Abstimmung aller Beteiligten sicherzustellen“ (ebd.). Verwaltungsstrukturell besteht der Unterschied zur oben skizzierten zweiten diskursiven Formation, dass Organisationseinheiten, die sich an Zugewanderte wenden, systematisch mit den Regelstrukturen verbunden sind und auf eine rasche „Übergabe“ ausgerichtet sind.

\subsubsection{Exkurs: Der Begriff „Integration“ aus diskurstheoretischer Perspektive}

Eine bedeutende Rolle in der Diskurstheorie von Laclau und Mouffe spielt die Verknüpfung von Diskurs und Hegemonie, die an der Unterscheidung von Differenz- und Äquivalenzketten ansetzt: Es gilt somit „zwischen einer ,Logik der Differenz' und einer ,Logik der Äquivalenz' zu unterscheiden: Die Logik der Differenz charakterisiert jeglichen Diskurs - ein Diskurs besteht aus einer Vielzahl miteinander verwobener Unterscheidungen. Beispielhaft ist hier etwa das Feld von Interessensgruppen innerhalb einer liberalen Demokratie, in der verschiedenste Differenzen nebeneinander und gegeneinander existieren, sich überschneiden und dabei uneinheitliche Fraktionierungen ergeben (etwa die voneinander unabhängigen Differenzen zwischen Arbeitnehmern und Arbeitgebern, zwischen Frauen und Männern, zwischen Stadt- und Landbewohnern etc.). Im dem Moment, in dem die Logik der Äquivalenz dominant wird, sieht sich diese Vielzahl von Unterscheidungen durch eine übergreifende, notwendig simplifizierende Identifizierung überformt: diese Identifizierung kann nur durch die Konstruktion eines Außens, eines radikal Anderen gelingen, im Verhältnis zu dem sich die Vielzahl einander überschneidender Differenzen zu einer übergreifenden Identität vereinheitlichen lässt, in der sie alle einander ,äquivalent' erscheinen. So lassen sich beispielsweise die genannten Differenzen zwischen Arbeitnehmern/ Arbeitgebern, Frauen/ Männern etc. über eine Identifizierung einer ,Nation“ oder ,Zivilisation` und durch eine 
Abgrenzung vom grundsätzlichen Außen des Barbarischen, des Fremden etc. aufheben" (Reckwitz 2006: S. 344).

Die Rolle, Differenzen zu überbrücken und einen hegemonialen Diskurs zu etablieren, kommt nach Laclau/Mouffe dem „leeren Signifikanten“ zu. „Genau diese universelle Aufgabe als allgemeines Äquivalent erfordert aber dessen weitgehende Entleerung von partikularen Inhalten (je umfassender die Kette, desto leerer der Signifikant). Das heißt, einerseits handelt es sich um einen ganz gewöhnlichen partikularen Signifikanten aus der Reihe aller Signifikanten einer Äquivalenzkette, andererseits signifiziert dieser Signifikant eben nicht allein etwas Partikulares, sondern er signifiziert die Einheit dieser Kette als solche, mit anderen Worten: das Prinzip der Kohärenz einer diskursiven Formation“" (Marchart 2017: S. 58). Ein partikulares Element eines Diskurses übernimmt somit die Repräsentation des gesamten Diskurses - hierzu muss er sich von konkreten Bedeutungen entleeren, damit er tatsächlich „alles“ repräsentieren kann (vgl. hierzu Begriffe wie „Freiheit“, „Nation“, „Gleichheit“ oder auch „soziale Marktwirtschaft"). Wie in Abschnitt 3.1 gezeigt werden konnte, bietet sich der Begriff „Integration“ gerade durch seine unspezifische Verwendung als „leerer Signifikant" an. Der Begriff muss unspezifisch sein, damit er eine Vielzahl von Praktiken/Diskursen zulässt. „Dabei ist die Deutungshoheit über den Signifikanten ,Integration“ umkämpft. So lässt sich verstehen, dass die einen im Namen der Integration staatliche oder institutionelle Diskriminierung kritisieren, während die anderen diese - ebenfalls im Namen der Integration - rechtfertigen und für ihre Verschärfung argumentieren. Der Begriff funktioniert zeitgleich als emanzipatorische Forderung und als Regierungstechnik“ (Buck 2011: S. 30). „Integration“ fungiert als leerer Signifikant und vereint unterschiedlichste Interessen und Diskurse gegenüber der (historisch scheinbar überholten) politischen Parole: Deutschland ist kein Einwanderungsland. „Unter dem Banner des Signifikanten ,Integration' vereinen sich unterschiedlichste politische Projekte. In diesem Sinne kann ,Integration' als leerer Signifikant gelten“ (ebd.: S. 41f.). Nonhoff spricht von „hegemonialen Artikulationen zweiter Ebene“, wenn in SubDiskursen um die „richtige“ Auslegung des leeren Signifikanten gestritten wird (Nonhoff 2006: S. 204). Es bilden sich konkurrierende Äquivalenzketten und es geht um eine „antagonistische Kooperation“, „im Zuge derer um die Ausgestaltung des Artikulationsmusters jenseits eines artikulatorischen Kerns (um den leeren Signifikanten) Konkurrenz herrscht" (ebd.: S. 205). 


\subsubsection{Der „Brückenschlag“ zum Feld sozialer Hilfe(n)}

Solange die Teilhabeberechtigung mit dem Aufenthaltsstatus verknüpft ist, werden die Kämpfe überwiegend durch den Einsatz juristischen und administrativen Kapitals geprägt. Im Rahmen von „Präsenzregime“-Strategien wird es aber auch möglich, über die Neuausrichtung von Diskursen und Praktiken die Brücke zum Feld sozialer Hilfen zu schlagen. Denn erfolgt eine Trennung zwischen „Mobilitäts-“ und „Präsenz“-Diskurs, wird der Anschluss des Feldes der Zuwanderungspolitik an das Feld sozialer Hilfen erleichtert, das auf diese Weise in das Feld der Zuwanderungspolitik „hineingezogen“ wird, womit es zu spezifischen Überlappungen kommt: „In diesem Kontext ist es hilfreich, grob zwischen Institutionen zu unterscheiden, die sich spezifisch an Migrantinnen und Migranten im Allgemeinen oder Teile zugewanderter Bevölkerung richten (z. B. Integrationspolitik, Ausländerbehörden, Asylpolitik, Härtefallkommissionen) und solchen, die wie Jugendhilfe, Bildungsinstitutionen, sozialer Wohnungsbau oder Arbeitsmarktpolitik ihre Klientel primär nach anderen Maßgaben identifizieren, sich aber institutionell auch mit migrationsbezogenen Problematisierungen auseinandersetzen. Die Diffusion von migrationsund diversitätspolitischen Problematisierungen in die öffentlichen Verwaltungen und den öffentlichen Dienstleistungssektor erreichte in der sogenannten Flüchtlingskrise, in deren Folge sie in fast allen gesellschaftlichen Bereichen gleichzeitig thematisch wurde, einen vorläufigen Höhepunkt. Aus der Perspektive von Migrationsregimen sind insbesondere das Zusammenspiel der Akteure und Institutionen, ihre charakteristischen Konvergenzen und Divergenzen sowie ihre blinden Flecken und wechselseitigen Verstärkungen interessant" (Nieswand 2018: S. 96).

Diesen Gedanken kann man in einer Erweiterung der Perspektive auch feldtheoretisch fassen. „Teilhabeberechtigung“ weist eine ähnlich doppelte Bedeutung auf wie „Teilhabe“ selbst - als Berechtigung zur Teilhabe an gesellschaftlichen Feldern und als Berechtigung zur Teilhabe an den Feldern sozialer Hilfe. In den „Überlappungszonen“ kommt es dann auch nicht selten zu Kämpfen im Sinne Bourdieus, in denen es einerseits darum geht, welche Regeln und Normen welchen Feldes gelten, andererseits um die Frage, wie durch diese Geltung die Regeln des jeweiligen Feldes verändert werden. Nach Nieswand „sind besonders jene Fälle interessant, in denen sich Neukonfigurationen von Feldern aus situativen Überlappungen von ,Rechtskreisen' und Zuständigkeiten ergeben. In diesen Fällen müssen Priorisierungen und Arbeitsteilungen von den Akteuren vorgenommen werden, um einen Arbeitskonsens herbeizuführen, wer an welcher Stelle 
des Verfahrens aktiv wird, wer für welchen Teilaspekt verantwortlich ist und wessen Zuständigkeitsbereich mehr Gewicht zugemessen wird. So kann für den Fall des Umgangs mit Unbegleiteten minderjährigen Flüchtlingen (UMF) in Deutschland gezeigt werden, wie die konkurrierenden institutionellen Kontexte der Jugendhilfe einerseits und der Asyl- und Ausländerverwaltung andererseits eine Spannung erzeugt haben, die - je nach lokalem Kontext oder Bundesland - lange Zeit zu unterschiedlichen Umgangsformen, insbesondere mit 16- und 17-Jährigen unbegleiteten Geflüchteten, geführt hat. Während diese Personen nach dem Asylverfahrensgesetz als voll verfahrensfähig galten, wurden sie im Rahmen der Jugendhilfe als besonders schutzbedürftige Minderjährige verstanden. Erst in den letzten Jahren setzte sich bundesweit die Priorisierung des Rechts- und Institutionenkreises der Jugendhilfe durch" (ebd.: S. 99). Je bedeutsamer das „Präsenzregime" 19 in Relation zum „Mobilitätsregime“ wird, umso größer und zahlreicher werden die „Überlappungszonen“, umso häufiger wird das Feld sozialer Hilfen mit Fragen und Folgen der Zuwanderung konfrontiert. Der unter der Sinnformel „Integration“ geführte Diskurs um die Notwendigkeit, Teilhabechancen zu sichern und Teilhabekompetenzen zu stärken, untermauert die Position von Sozialpolitik und Sozialer Arbeit gegenüber der Migrationspolitik. Gleichzeitig ist auf Bundesebene und auch in vielen Kommunen die Gegenbewegung spürbar, das "Präsenzregime“ einzuhegen und die „Teilhabeberechtigung“ für einen großen Teil der Zugewanderten in Frage zu stellen.

Wir haben es also im Feld der Zuwanderung und im Überlappungsbereich zum Feld der sozialen Hilfe mit einer komplexen und dynamischen Gemengelage zu tun, in der sich zwei Spannungsfelder identifizieren lassen:

Das dynamische Verhältnis zwischen Mobilitäts- und Präsenzregime, das sich z. B. in den Auseinandersetzungen um „Duldungen“ zeigt (vgl. die „Ausbildungsduldung“):

1) Das Zusammenspiel von Mobilitäts- und Präsenzregime unterliegt einem historischen Wandel. Zunächst konstituierte sich das Mobilitätsregime, während das Präsenzregime bis in die 1990er-Jahre hinein eher rudimentär blieb. Deutlich wird dies am AsylbLG. Dies gewährt auch Personen ohne oder mit ungeklärtem Schutzstatus eine eingeschränkte Teilhabeberechtigung. Sie erhalten materielle Leistungen, Leistungen des Bildungs- und Teilhabepakets und eine medizinische Grundversor-

19 Wir verwenden diese Begriffe als Abkürzung für den oben dargestellten feldtheoretischen Hintergrund. 
gung. Komplexe Regelungen ermöglichen unter bestimmten Bedingungen den Zugang zum Arbeitsmarkt bzw. zu arbeitsmarktbezogenen Dienstleistungen nach SGB III. Da das AsylbLG nicht Bestandteil des Sozialgesetzbuches ist, besteht allerdings kein Anspruch auf (personenbezogene) Dienstleistungen ${ }^{20}$, ist also der Übergang ins Feld sozialer Hilfen blockiert. Für den eng gezogenen Kreis der Flüchtlinge mit „guter Bleibeperspektive" wird unter bestimmten Umständen der Zugang zu Integrationskursen eröffnet, dem einzigen Element des Präsenzregimes, das in das Mobilitätsregime „hineinragt“ (vgl. Schaubild 4).

\section{Schaubild 4: Mobilitäts-und Präsenzregime}

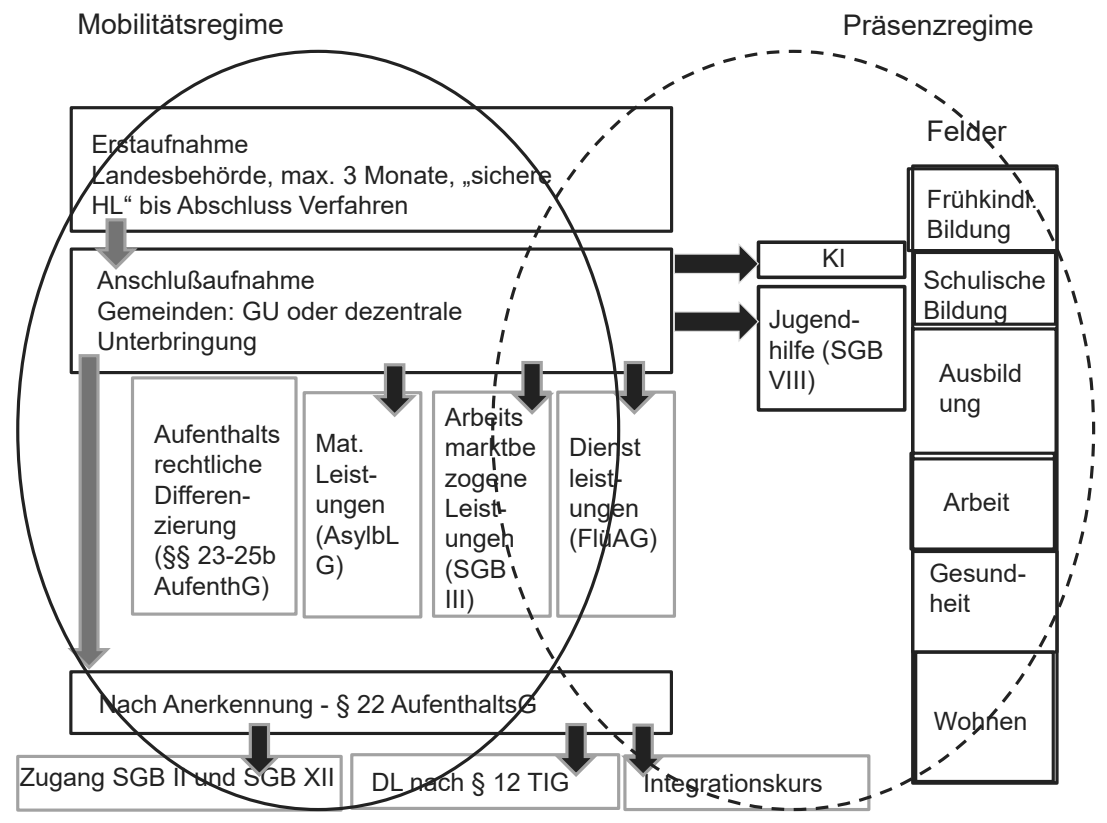

(Eigene Darstellung)

Eine Betrachtung des Zusammenspiels von Aufenthalts- und Leistungsrecht zeigt somit eine erhebliche Spaltung zwischen den „Rechtskrei-

20 Ausgenommen sind hier die unbegleiteten minderjährigen Ausländerinnen und Ausländer, die von Beginn an Leistungen nach dem SGB VIII erhalten. Sie werden deshalb in die weitere Betrachtung nicht systematisch mit einbezogen. 
sen“ (AsylbLG/SGB III - SGB II/SGB XII) im Zugang zu Dienstleistungen (vgl. Schaubild 5).

\section{Schaubild 5: Personenbezogene Dienstleistungen}

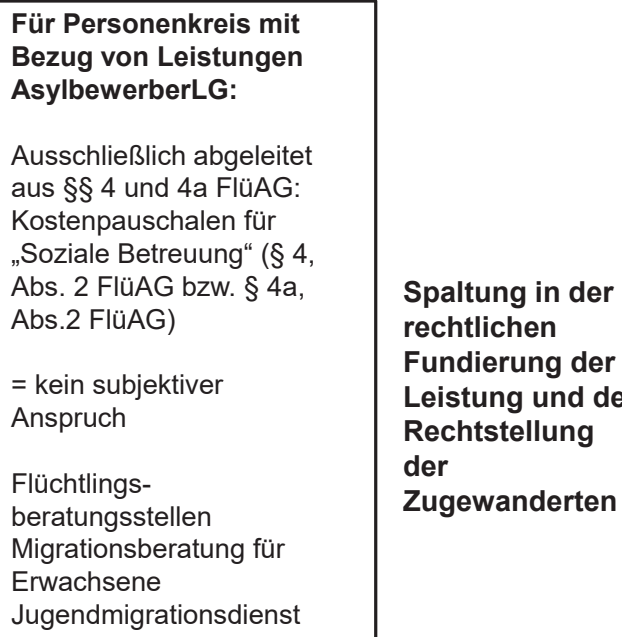

Ausschließlich abgeleitet aus $\S \S 4$ und 4a FlüAG: Kostenpauschalen für "Soziale Betreuung" (§ 4, Abs. 2 FlüAG bzw. § $4 a$, Abs.2 FlüAG)

$=$ kein subjektiver

Anspruch

Flüchtlings-

beratungsstellen

Migrationsberatung für

Erwachsene

Jugendmigrationsdienst

Rechtstellung

der

Zugewanderten

Für Personenkreis nach § 22 bzw. § 23, Abs. 2 AufenthaltsG:

Aufgaben nach $\S 12$, Abs. 1 Teilhabe- und Integrationsgesetz NRW = direkte

Aufgabenverpflichtung der Verwaltung

Ggf. Leistungen nach §§

14 und 16a SGB II =

subjektiver Anspruch
Flüchtlings-

beratungsstellen

Migrationsberatung für

Erwachsene

Jugendmigrationsdienst

(Eigene Darstellung)

2) Die „Überlappungszone“ zum Feld sozialer Hilfen. Über den Kampf zwischen Akteuren des Mobilitäts- bzw. Präsenzregimes werden der rechtliche Status der Zugewanderten und damit auch die „Teilhabeberechtigung" an den einzelnen gesellschaftlichen Feldern definiert. Hierzu gehört auch das Feld sozialer Hilfen als „stellvertretender Inklusion", da zwar eine Minimalabsicherung und damit eine - wenn auch prekäre - Teilhabe an Gesellschaft gesichert ist, aber auf einem für bestimmte Gruppen abgesenktem Niveau (Asylbewerberleistungsgesetz) und bedroht durch Praktiken des Mobilitätsregimes (Abschiebung). Damit wird ein gegliederter Raum innerhalb des Territoriums der Bundesrepublik geschaffen, der über die durch den Aufenthaltsstatus geregelten „Teilhabeberechtigungen“ für Teilhabechancen definiert wird. Mit Blick auf das Aufenthaltsrecht lässt sich feststellen, dass unabhängig von der Differenz zwischen formalen und substanziellen Rechten bereits hinsichtlich der formalen Rechte von „civic stratification“, einer staatlich intendierten Abstufung von Rechten und einer damit einhergehenden Stratifizierung der Bevölkerung gesprochen werden kann 
(vgl. Hess/Lebuhn 2014: S. 18). Zugang zu zentralen Funktionssystemen ist damit für Zugewanderte doppelt selektiv: Durch die staatliche Regulierung gestaffelter Teilhabeberechtigung und durch die immanenten Regeln der gesellschaftlichen Felder, Teilhabechancen zu gewähren.

Wenn die Logik des Präsenzregimes auf die lokalen Systeme sozialer Hilfen ausgedehnt wird, kann dies zu einer „Irritation“ autonomer Felder in ihren Regelsystemen und ihrem institutionellen Gefüge führen. Routinen werden in Frage gestellt, Räume für Diskurse eröffnet, die Veränderungen des organisationalen Habitus induzieren können. Die Ausweitung des Präsenzregimes im Zuge des Einwanderungsmanagement kann also dazu führen, dass immer mehr Institutionen und Praktiken im System der sozialen Hilfe mit einer Zielgruppe konfrontiert sind, die besonderer Unterstützung beim Ausbau ihrer Teilhabekompetenzen bedarf und für die in spezifischer Weise Teilhabechancen erhöht werden müssen (Anerkennung von Abschlüssen, Veränderungen im Bereich schulischer und beruflicher Bildung etc.). Es ist offen, wie sich dies auf das System der Hilfe auswirken wird: ob es zur „Einklammerung“ in Sonderzuständigkeiten kommt, strukturelle Probleme als „Einzelfälle“ einer routinisierten Bearbeitung zugeführt werden oder sich das System verändert, indem der Zusammenhang zwischen dem Feld der Zuwanderungspolitik und dem Feld sozialer Hilfen neu definiert wird, indem Übergänge systematisch gestaltet und in Einzelfällen begleitet werden (z. B. durch Produktionsnetzwerke und „Teilhabemanagement"). An dieser Stelle erhält das lokale Feld eine besondere Bedeutung: es hängt nicht zuletzt von lokalen Diskursen ab, ob und wenn ja welche Lösungen für diese strukturelle Problematik gefunden werden: "Sonderzuständigkeiten“, zusätzliche Organisationseinheiten und/oder Steuerungsgremien, Entwicklung übergreifender Standards für die Leistungsgewährung für alle - oder: nichts, d. h. Fortführung der tradierten Spaltung, aufgelockert durch die Bearbeitung offensichtlicher Defizite.

Wir stoßen also erneut auf die Bedeutung der lokalen Felder. Hier lässt sich dann konkret beobachten, mit welchen Diskursen und Praktiken die Akteure des Feldes sozialer Hilfe auf die neue Konstellation reagieren, ob sich das Feld der Zuwanderungspolitik wie in der Vergangenheit als abgeschottetes Feld mit starkem administrativen (Ausländerbehörde, Sozialamt) und schwachem sozialpädagogischen Pol (Beratungsdienste) darstellt oder ein „Brückenschlag“ die Gewichte verändert. Auf alle Fälle kommt den Kommunen eine zentrale Rolle in der Ausgestaltung des Leistungsangebots für Zugewanderte und auch für die Strukturierung des „Rechtskreiswechsels“ zu. Deshalb ist es nicht verwunderlich, dass die (wenigen) 
empirischen Untersuchungen zur kommunalen Praxis in diesem Teil des Feldes der Zuwanderung eine hohe Diversität zwischen den Kommunen zeigen (vgl. Aumüller/Bretl 2008; Schammann 2017; Schammann/Kühn 2017), sowohl was die Nutzung von Handlungsspielräumen im Bereich der Ausländerbehörden angeht wie auch hinsichtlich der Entwicklung übergreifender Vernetzungsstrategien und einer entsprechenden Praxis. Schammann nennt hierfür einige strukturelle Gründe: „So erreicht die Anzahl der Asylsuchenden in Mittel- und Großstädten schneller eine kritische Masse, die nicht nur politischen Handlungsdruck erzeugt, sondern für die sich auch die Bereitstellung neuer Maßnahmen rechtfertigen lässt. Auch die Entfernung zwischen Unterkünften und Angeboten ist kürzer als auf dem Land. Außerdem haben Städte in der Regel häufig lange und kontinuierliche Erfahrung mit den Themen Migration und Integration und verfügen meist über gewachsene institutionelle Strukturen - manifestiert etwa im Amt eines kommunalen Integrationsbeauftragten oder in der regelmäßigen Erhebung statistischer Daten zur lokalen Bevölkerung mit Migrationshintergrund. Auch zivilgesellschaftliche Initiativen der Integrationsund Flüchtlingsarbeit sowie Migrantenorganisationen sind in großen Städten zahlreicher und kontinuierlicher präsent. Ihre Interessenartikulation ist meist über Integrationsbeiräte oder Runde Tische institutionalisiert. All dies sorgt für eine kontinuierliche Präsenz des Themas Migration in der Stadtpolitik, an die in der Flüchtlingspolitik, die von starken Schwankungen gekennzeichnet ist, angeknüpft werden kann. In kleineren Städten verschwindet das Thema Migration dagegen häufig für längere Zeit von der Agenda und wird in Zeiten großer (Flucht-)Zuwanderung jeweils wieder neu als lokalpolitisches Thema, entdeckt'. Die strukturellen Unterschiede zwischen Städten und kleineren Gemeinden führen dazu, dass man sowohl für die Erledigung von Pflichtaufgaben als auch für die Initiierung freiwilliger Aufgaben unterschiedliche Voraussetzungen annehmen muss" (Schammann 2017: S. 109f.). Michael Bommes hat aus einer vergleichenden Längsschnittanalyse der „Migrationspolitik“ von zwei Kommunen folgende Erkenntnis gewonnen, die auch aktuell für die Konstitution eines kommunalen Feldes der Zuwanderung gilt: „Migration und kulturelle Wandlungsprozesse sind keine vorgängigen sozialen Ereignisse, auf die kommunale Organisationen als externe Umweltereignisse reagieren. (...) In den Organisationen des politischen Systems sowie in den Organisationen anderer gesellschaftlicher Teilbereiche bilden sich erst die sozialen Strukturen, die als Migration registriert werden. Welche Art Geschehen Migration in einer Kommune und damit in diesem organisatorischen Teilkomplex des politischen Systems ist, welche kulturellen und sozialen Fol- 
gen damit für die Gemeinde verbunden sind, wird mit der Entstehung von Zuständigkeiten und Einrichtungen, mit der Beschreibung von Problemen, Lösungen und angemessenen Mitteln festgelegt. Was dann geschieht, wird auf der Folie solcher Strukturen registriert und weiter verarbeitet" (Bommes 2003: S. 478). Bei der Betrachtung kommunaler Zuwanderungspolitik interessiert somit nicht nur die Deskription der Diversität kommunaler Praxis, sondern vor allem die Analyse dieser Diversität vor dem Hintergrund der oben dargestellten feldtheoretischen Überlegungen. Denn es hängt von der kommunalen Politik ab, ob sich „Migration“ als abgegrenztes Feld etabliert (isolierte Spezialdienste) und die Felder der sozialen Hilfen Migrationsfolgen als „Randphänomene“ behandeln oder aber umgekehrt die Felder sozialer Hilfen reflexiv auf die mit Migrationsfolgen verbundenen Phänomene reagieren und sie mit einer nunmehr modifizierten „Feldlogik“ verarbeiten. Dies wird überwölbt von der landes- bzw. bundespolitischen Migrationspolitik, die aktuell - zumindest was letztere angeht -die Selektivität eher steigert als minimiert.

\subsection{Ein komplexes Verständnis von "Teilhabe“ als konzeptionelle Grundlage des Integrationsmanagements}

Die Unterscheidung von Teilhabechancen und Teilhabekompetenzen und ihre Integration im Konzept des "Capabilty Approach“ macht deutlich, dass Unterstützungsleistungen für Personen, deren Teilhabe gesichert werden soll, sowohl auf der individuellen Ebene (Teilhabekompetenzen) wie auf der "Systemebene“ (Teilhabechancen) ansetzen müssen. Die Sicherung von Teilhabechancen ist sicherlich zentral, muss aber eventuell ergänzt werden durch Hilfen auf der Ebene der „Teilhabekompetenzen“ - und diese Hilfen müssen sich auf die je individuelle Ausgangskonstellation von Hilfesuchenden beziehen, um deren Kompetenzen zu stärken, Ressourcen zu erschließen bzw. in Anspruch zu nehmen. Im 4. Kapitel wird ausgeführt, dass hierzu die einzelne Person im Vordergrund stehen muss, deren Geschichte aus unterschiedlichen Perspektiven als „Fall“ rekonstruiert wird. Umgekehrt laufen aber einzelfallbezogene Hilfen leer, wenn nicht auf der Systemebene „objektive“ Teilhabechancen gesichert werden. Dies gilt allgemein, besonders aber im Hinblick auf Zugewanderte. Für diese Gruppe gilt, dass vorgängig und parallel die „Teilhabeberechtigung“ gesichert werden muss.

Diese Aufgabe erfordert komplexe Aktivitäten im Hinblick auf die Bildung von Netzwerken (vgl. Kapitel 4) und die Ausgestaltung von Case Ma- 
nagement (vgl. Kapitel 5). In beiden Fällen müssen aufenthaltsrechtliche Fragestellungen (die elementar die Teilhabeberechtigung betreffen) immer schon mitgedacht bzw. mitverhandelt werden. 


\section{Ideen zur Gestaltung und zum Management multilateraler Kooperationsstrukturen (Produktionsnetzwerke) ${ }^{21}$}

\subsection{Vorbemerkung}

„Die Bedeutung von Unternehmensallianzen, also kooperativen Arrangements, als Organisationsform der Wertschöpfungsprozesse nimmt seit Jahren zu. Der Begriff ,Allianz' (...) hat jedoch bis heute keine einheitliche Definition und Interpretation erfahren (...). Zudem existiert eine Vielzahl von Termini wie ,Strategische Allianz', Joint Venture', ,Netzwerk', ,Strategic Partnership', ,Koalition', ,Collaborative Agreement', ,Bündnis', ,Wertschöpfungspartnerschaft, die meist synonym zur Beschreibung des gleichen Phänomens verwendet, teilweise jedoch voneinander abgegrenzt werden (...) “ (Morschett 2005: S. 379). Diese Feststellung aus einem betriebswirtschaftlichen Handbuch gilt auch heute noch und ebenso für andere Wissenschaftsdisziplinen. So werden die Begriffe „Allianz“ und „Bündnis“ vorzugsweise in der politikwissenschaftlichen Literatur verwendet (vgl. Köhling/Stöbe-Blossey 2018), der Begriff „Netzwerk“ scheint gleichsam unscharf wie universell zu sein.

In diesem Kapitel soll versucht werden, etwas Orientierung in diesem terminologischen Wirrwarr zu stiften, indem zwei soziale Sachverhalte, die eng miteinander verbunden sind, sich aber gleichzeitig voneinander unterscheiden, näher bestimmt und aufeinander bezogen werden: $\mathrm{Bi}$ - und multilaterale Kooperation zwischen Organisationen und organisationale Netzwerke. Dabei wird der Begriff „Netzwerk“ ganz bewusst gegenüber den Begriffen „Bündnis“, „Allianz“ o. ä. verwendet, weil er - wie erwähnt - universell verwendet wird und auch einen vergleichsweise breiten Begriffshorizont aufweist, was ihn für eine terminologische Differenzierung besonders geeignet macht. In Abschnitt 4.2 werden die beiden genannten Sachverhalte „Netzwerk“ und „Kooperation“ inhaltlich näher bestimmt und voneinander abgegrenzt. Dabei wird es insbesondere darum gehen, Netzwerke mit unterschiedlicher „Leistungstiefe“, d. h. verschiedenen Graden kooperativ gestalteter Verbindungen, zu unterscheiden. Im Zuge der wissenschaftlichen Auseinandersetzung mit Netzwerken und anderen For-

21 Dieses Kapitel basiert auf überarbeiteten Teilen der Publikation von Reis u.a. 2016. 
men interinstitutioneller Kooperation wurden unterschiedliche theoretische Ansätze entwickelt, um die mit diesen Begriffen verbundenen Phänomene besser zu verstehen. Mit ihrer Hilfe lassen sich jeweils verschiedene Dimensionen von „Netzwerken“ erfassen, eine Gesamtbetrachtung erfordert aber die Auseinandersetzung mit allen "Zugangsweisen“. Dies beinhaltet die Rekonstruktion eines theoretischen Instrumentariums, das geeignet ist, das Phänomen „organisationales Netzwerk“ möglichst umfassend analytisch zu begreifen (Abschnitt 4.3). Das hier vorgestellte Konzept des „Produktionsnetzwerks“ basiert auf konstruktivistischen Theorieansätzen und fokussiert auf die Diskussion und Erarbeitung von „Fällen“ als Ausgangspunkt der Netzwerkbildung (Abschnitt 4.4). Auf dieser Grundlage können „Gelingensbedingungen“ für Produktionsnetzwerke und die Voraussetzungen benannt werden, die vorhanden sein müssen, damit bestimmte Erwartungen, die mit „Netzwerken“ verknüpft werden, auch erfüllt werden können (Abschnitt 4.5). Die Erarbeitung von neuen Leistungsangeboten und/oder -prozessen in einem Netzwerk erfordert unter Umständen einen „langen Atem“, vor allem aber einen systematisierten Ablauf. Welche Schritte hierfür notwendig sind, wird in Abschnitt 4.6 dargestellt. Werden in Netzwerken Leistungsprozesse verbindlich miteinander verknüpft, kann von „Dienstleistungsketten“ gesprochen werden. Wie diese aussehen könnten und wie sie visualisiert werden, zeigt Abschnitt 4.7. Netzwerke benötigen ein professionelles Netzwerkmanagement, zumindest dann, wenn die interne Kooperation besonders ausgeprägt und in hohem Maße verbindlich ist. Wie ein solches Netzwerkmanagement aussehen könnte, wird in Abschnitt 4.8 dargestellt. In Abschnitt 4.9 findet sich ein Beispiel aus einer Kommune, die am Projekt teilgenommen hat.

\subsection{Kooperation und Netzwerke}

\subsubsection{Begriffliche Klärungen}

In der einschlägigen Literatur wird häufig zwischen „Kooperation“, „Koordination“ und „Vernetzung“ unterschieden, um Stufen der Intensität des Zusammenwirkens systematisch zu unterscheiden (vgl. z. B. Kardorff 1998; van Santen/Seckinger 2005; Corbett/Noyes 2008). Van Santen und Seckinger fassen unter „Kooperation ein Verfahren (...), bei dem im Hinblick auf geteilte oder sich überschneidende Zielsetzungen durch Abstimmung der Beteiligten eine Optimierung von Handlungsabläufen oder eine 
Erhöhung der Handlungsfähigkeit beziehungsweise Problemlösungskompetenz angestrebt wird. (...)“ (Van Santen/Seckinger 2003: S. 29). Eine zentrale Voraussetzung von Kooperation ist somit, dass sich Ziele, Aufgaben oder Interessen von mehreren Beteiligten zumindest partiell zur Deckung bringen lassen. Unter Umständen muss hierzu das Handeln verschiedener Akteure aufeinander abgestimmt bzw. koordiniert werden. „Koordination" heißt dann, dass die Handlungen von zwei oder mehreren Akteuren auf ein Ziel ausgerichtet und systematisch miteinander verknüpft werden (vgl. Schubert 2011: S. 532) - was über Kooperation hinausgeht.

Strukturen der Vernetzung in diesem Sinne weisen Ähnlichkeiten zu Organisationen auf: Um (gemeinsame) Ziele verfolgen und erreichen zu können, werden Spielregeln und Strukturen geschaffen. „Successful models of inter-agency cooperation tend to be governed by a detailed, clearly defined strategy and remit, a commitment to shared objectives and clear targets informed by an overarching strategic vision" (Lindsay/McQuaid/ Dutton 2008: S. 719). Das bedeutet umgekehrt, dass Vernetzung nicht funktionieren kann, wenn es solche Gemeinsamkeiten nicht gibt bzw. sie von den Beteiligten nicht gesehen werden. „Wenn alle Beteiligten eine Aufgabe bearbeiten, die zur Erreichung des gemeinsamen Ziels beiträgt, kann ,symbiotic inter-dependency entstehen - aus der Kooperation entsteht Nutzen für alle Beteiligten“ (Ludwig 2013: S. 13). Eine Paradoxie der Vernetzung besteht darin, dass der Nutzen der Zusammenarbeit für die Beteiligten gerade aus den Unterschieden der Einzelorganisationen in Ressourcen und Expertise herrührt. Diese Unterschiede ergeben sich aber aus den unterschiedlichen Zielsetzungen der Organisationen. Jede Organisation sucht andere Vorteile in der Zusammenarbeit (vgl. Huxham/Vangen 2005: S. 82), dennoch müssen sie ein Minimum an Überschneidung realisieren. Vernetzung oder ein "Netzwerk“ als deren institutionalisiertes Resultat stellt somit einen Spezialfall von Kooperation dar - allerdings nur dann, wenn man das Kontinuum von Zusammenarbeit untersucht, also ein Minimum von Verbindlichkeit zum Ausgangspunkt nimmt.

Das US-amerikanische Forschungsteam Thomas Corbett und Jennifer Noyes hat ein Modell der Intensität von Kooperationsbeziehungen entwickelt (vgl. Corbett/Noyes 2008: S. 10). Dieses spannt sich zwischen der „Kommunikation“, verstanden als (regelmäßigem) Informationsaustausch, und der vollen Integration ursprünglich getrennter Leistungen zu einem einheitlichen Leistungsprozess unter einem organisationalen Dach:

- Kommunikation: formaler, nicht bewertender Informationsaustausch.

- Kooperation: gegenseitige Unterstützung durch Informationen und Berücksichtigung der Inhalte und Angebote. 
- Koordination: gemeinsame Aktivitäten, intensivere Kommunikation, individuelles Engagement bezüglich gemeinsamer Ziele und Planungen.

- Zusammenarbeit: die beteiligten Dienste, Personen oder Gruppen sind bereit, Teile ihrer Eigenständigkeit zugunsten gemeinsamer Ziele aufzugeben. Ernst gemeinte Zusammenarbeit erfordert Veränderungen in der Organisation und im Verhalten im Dienste gemeinsamer Ziele und Ideale.

- Konvergenz: Neustrukturierung der Angebote und Programme, Budgets, Zielstellungen und Personalkonzepte.

- Vertiefung: die beteiligten Dienststellen, Ämter, Geschäftsstellen etc., das Verhalten ihres Personals, ihre Aktivitäten, Strategien und Ressourcen sind vollständig zusammengeführt und aufeinander abgestimmt. Individuelle Autonomie oder Ziele treten zugunsten der gemeinsamen Identität vollständig in den Hintergrund.

Die Ausdifferenzierung unterschiedlich intensiver Formen der Kooperation kann zwei Zwecken dienen:

- Pragmatisch der Entscheidung, welche Intensität tatsächlich notwendig ist, um bestimmte „vernetzte“ Leistungen zu realisieren. Dabei sollte sich diese Entscheidung am gewünschten „Outcome“ orientieren und nicht an vorgegebenen Standards (vgl. ebd.: S. 12f.).

- Perspektivisch der Abschätzung, welche Schwierigkeiten bei der Neukonstruktion von Dienstleistungsprozessen zu erwarten sind, wenn ein bestimmter Grad an Kooperation angesichts der spezifischen Organisationskultur der kooperierenden Organisationen erreicht werden soll.

Netzwerke ${ }^{22}$ sind mehr und anderes als Kooperation - je nach theoretischer Akzentuierung. Der Begriff des „Netzwerks“ ist nämlich trotz langjähriger wissenschaftlicher Thematisierung nach wie vor sehr schillernd, was nicht alleine seinem metaphorischen Charakter geschuldet ist - „Man ahnt ungefähr, wovon die Rede ist" (Baitsch/Müller 2001: S.9). Je nach fachwissenschaftlicher Perspektive geraten andere Aspekte und Konstellationen von Akteuren ins Zentrum der Betrachtung, was eine präzise einheitliche Verwendung sehr erschwert. Bommes/Tacke weisen deshalb zu Recht darauf hin, dass der „soziologische Strukturbegriff“ von einer vor-

22 Die vorliegende Ausarbeitung beschäftigt sich ausschließlich mit Netzwerken, die von Organisationen gebildet werden („tertiäre“ Netzwerke). Aus diesem Grund fallen "primäre Netzwerke" wie Verwandtschaften, Freundeskreise und „sekundäre Netzwerke“ (Verbindungen von Individuen zu Unterstützung leistenden Institutionen, vgl. Schubert 2005) aus der Betrachtung. 
wiegend semantischen Verwendung getrennt werden sollte, „die dem Netzwerkkonzept eine je system- und konjunkturspezifische Bedeutung verleiht“ (Bommes/Tacke 2006: S. 38). Sprechen die einen von „Adressen“ (vgl. Tacke 2000), um damit den Unterschied zu Organisationen zu markieren, so sind für die anderen Netzwerke Formen der Kooperation wie z. B. auch Zuliefererbeziehungen oder Produktionscluster (vgl. Sydow/ Möllering 2009).

Im Kontext der Betriebswirtschaft stehen Versuche, Netzwerke als „Unternehmensnetzwerke" zu betrachten, die einen teilweise hohen Grad an Verbindlichkeit aufweisen (vgl. grundlegend Windeler 2001), je nachdem, ob es sich um „strategische“ oder „regionale Netzwerke“ handelt. Letztere werden von Baitsch/Müller als eine Art „Möglichkeitsraum“ angesehen, der die Grundlage für eine verbindliche Kooperation zwischen Akteuren bildet. Netzwerke bilden die Voraussetzung für realisierte Kooperationen, sind aber nicht mit diesen identisch, sondern lassen mehr Optionen zu, als tatsächlich wahrgenommen werden (vgl. Baitsch/Müller 2001). „Kooperation" bezeichnet nach dieser Auffassung somit ein gemeinsames Handeln organisationaler Akteure, während „Netzwerk“ einen Strukturbegriff darstellt. Windeler versucht die Vermittlung von Struktur und Handeln im Hinblick auf die Netzwerktheorie, indem er im Rückgriff auf die Strukturationstheorie von Anthony Giddens verdeutlicht, dass die (Un-)Verbindlichkeit von Netzwerken Resultat des Handelns von den entsprechenden Akteuren ist, wie sie umgekehrt dessen Reichweite absteckt (vgl. Windeler 2001). Damit ist gleichzeitig die Dynamik der Netzwerkbildung in den Blick genommen - Netzwerke können gleichzeitig als „Möglichkeitsräume" (für Zukünftiges) wie als Struktur realisierter multilateraler Kooperation (für die Gegenwart) begriffen werden. Diese Differenzierung wird unten in der Unterscheidung zwischen Produktions- und Projektnetzwerk aufgegriffen. Politikwissenschaftlich werden Netzwerke als Systeme überwiegend informeller, aber auch formeller, horizontaler und hierarchischer Beziehungen zwischen (halb-)autonomen institutionellen Akteuren definiert, die mit unterschiedlichen, aber wechselseitigen Interessen ein als "gemeinsam“ definiertes Problem bearbeiten (vgl. Hild 1997). „Bearbeitung“ kann dabei sowohl Interessensaustausch, gemeinsame Interessensformulierung, aber auch „Produktion eines Kollektivgutes" bedeuten (vgl. Benz 1995). Das zentrale Merkmal organisationaler Netzwerke ist dabei „die Entstehung eines mehr oder weniger stabilen Musters von Beziehungen zwischen autonomen Akteuren" (Strassheim/Oppen 2006: S. 18). Netzwerke sind kein Selbstzweck, sondern werden geschaffen, weil sich alle „autonomen Akteure“ Vorteile versprechen (Win-win-Situation - ob diese 
tatsächlich eintritt, ist eine empirisch zu klärende Frage): „Kooperative Strategien erfordern Investitionen im Sinne von Zeit, Personal und Informationen, die sich aus Perspektive der beteiligten Akteure nur rechtfertigen, wenn die eigenen Ziele durch Kooperation besser (effizienter und effektiver) erreicht werden können. (...) Ressourceninterdependenz ist der theoretische und praktische Kern von Netzwerken“ (ebd.).

Über beide Zugangsweisen können folgende zentrale Elemente organisationaler Netzwerke bestimmt werden:

- Es besteht aus autonomen Akteuren,

- die in "reflexiver Koordination“,

- aber auch in Verfolgung eigener Interessen

- Strukturen ausbilden, die einen unterschiedlichen Grad an Verbindlichkeit haben können.

Wie „eng“, d. h. verbindlich, die Strukturen von Netzwerken jeweils sind, hängt wesentlich von den Erwartungen der einzelnen Akteure, aber auch vom „kollektiven Willen“ des Netzwerks ab.

Netzwerke können als Struktur und als Prozess betrachtet werden, dessen Zusammenhalt über die Reziprozitätserwartungen der einzelnen Akteure gestiftet wird, d. h. die auf Vertrauen gestützte Erwartung, für die Beteiligung am Netzwerk mittel- oder langfristig einen „Gewinn“ erzielen zu können. Die damit gesetzte Grundproblematik eines „tertiären Netzwerks“ (vgl. Schubert 2008: S. 38f.) kann im Anschluss an die Systemtheorie formuliert werden. Nach der Definition von Windeler sind Unternehmensnetzwerke nämlich „soziale Systeme, die vornehmlich aus Geschäftsinteraktionen und -beziehungen zwischen (autonomen) Unternehmungen zusammengesetzt sind, die diese überwiegend mit Blick auf den Beziehungszusammenhang zwischen sich reflexiv koordinieren" (Windeler 2001: S. 200). Ein funktionierendes Netzwerk bildet ein soziales System, das eine eigene Identität, eigene Rationalitäten und eine eigene Handlungsfähigkeit besitzt. Bestehende Organisationen als autonome organisationale Systeme verbinden sich zu einem „kollektiven Akteur“, der ein eigenes, emergentes System darstellt. Ein solches Netzwerk entwickelt eigene Kommunikations- bzw. Steuerungsmedien. Organisationale Netzwerke können deshalb als soziale Systeme mit eigenen Charakteristika und eigener „Handlungslogik“ begriffen werden. Charakteristika sind: Der dauerhafte Beziehungszusammenhang zwischen Organisationen (z. B. Unternehmen) und die Koordination ohne einheitliche Leitung, wie sie z. B. in Organisationen gegeben ist (vgl. ebd.: S. 242), die Handlungslogik ist die der „Verhandlung" (vgl. Hild 1997: S. 97) - allerdings setzt diese wie in einer spezifischen Interaktionsstruktur auf Reziprozität. 


\subsubsection{Netzwerktypen}

Die bisherigen Ausführungen machen deutlich, dass organisationale Netzwerke als fragile Gebilde anzusehen sind, deren Stabilität immer wieder durch geeignete (d. h. größtenteils gemeinsam getragene) Aktivitäten gesichert werden muss. Diese Aktivitäten müssen sowohl die Identität des Netzwerks als auch die der beteiligten Organisationen (d. h. deren „Autonomie“) im Auge behalten. Dies gilt auch für Netzwerke, die mit anderen Termini wie „Allianz“, „Bündnis“ o. ä. bezeichnet werden.

Die Identität des Netzwerks ist abhängig von zwei Faktoren: Der Beziehungsstruktur der Netzwerkpartnerinnen und -partner untereinander, die eher hierarchisch oder eher heterarchisch geprägt sein kann, und der inhaltlichen Ausrichtung: Richtungsoffen oder zielorientiert.

\subsubsection{Hierarchische und heterarchische Netzwerke}

Bevor hierarchische und heterarchische Netzwerke unterschieden werden, muss noch einmal die terminologische Unterscheidung zwischen Kooperationsbeziehungen und Netzwerken aufgegriffen werden.

Häufig bestehen - gerade im Falle politisch oder ökonomisch dominanter Organisationen - rege Austauschbeziehungen zwischen diesen Organisationen und diversen „Partnerorganisationen“, die allerdings untereinander nicht verbunden sind. Aus der Sicht der „zentralen“ (oder auch „fokalen“) Organisation bestehen Netzwerkbeziehungen, sie hat „ihr“ Netzwerk. Allerdings sind die Beziehungen der Beteiligten untereinander nur über diese zentrale Organisation vermittelt - es handelt sich um ein „indirektes Netzwerk“, das geprägt ist von bilateralen Kooperationsbeziehungen. In „direkten Netzwerken“ stehen demgegenüber viele (oder gar alle) Beteiligten in Kontakt zueinander. Unabhängig von dieser Unterscheidung können sowohl direkte als auch indirekte Netzwerke eher hierarchisch oder eher heterarchisch strukturiert sein (vgl. Kohlmeyer/Mauruszat/Seyfried 2000: S. 13f.):

1. Ein zentraler Akteur steuert und organisiert das Zusammenspiel der verschiedenen Akteure, ohne dass diese untereinander in Beziehungen treten, obwohl sie alle in Beziehungen zu einem Akteur stehen. Es handelt sich hier weniger um ein „Netzwerk“ als vielmehr um eine Ansammlung bilateraler Kooperationsbeziehungen, die auf einen dominanten Akteur (fokales Unternehmen) zentriert sind, z. B. die Beziehung zwischen einem Automobilwerk und seinen Zuliefererbetrieben. 
Das Problem dieses „Netzwerks“ liegt auf der Hand: Die Entwicklung wird stark von einem Akteur geprägt, die anderen befinden sich in einer partiell abhängigen Position. Dies kann die Innovationsfähigkeit gefährden. Aber auch dieser Typus stellt unter der Voraussetzung ein „Netzwerk" dar, dass sich die nicht-fokalen Organisationen nicht in völliger Abhängigkeit vom fokalen Unternehmen befinden, ihre „Organisationsdomäne“ sich somit nicht vollständig mit der „Netzwerkdomäne" deckt (vgl. Sydow/Möllering 2009: S. 215). In diesem Fall reicht der Aktionsradius der am Netzwerk beteiligten Akteure über den Netzwerkzusammenhang hinaus und es kommt zur Entwicklung wirklich „kollektiver Strategien“ (und nicht zur Durchsetzung der Strategie des fokalen Unternehmens wie im Falle von Abhängigkeit).

2. Hier steht ein Akteur im Mittelpunkt, er hat jedoch keine übergeordnete Steuerungsfunktion. Auch dabei handelt es sich um eine Ansammlung bilateraler Kooperationsbeziehungen. Dieser Typus stellt ein Gerüst aus langfristigen Kooperationen dar, die ein einzelner Akteur zur Erfüllung seiner Aufgabe benötigt, ohne dass er diese dominiert. Das Problem dieses Typs besteht darin, dass es keine Gewähr für die Stabilität von Kooperationsbeziehungen gibt, was bereits mittelfristige Planungen erschwert.

3. Bei diesem Typus eines hierarchischen Netzwerks wird die Steuerung, die durch den zentralen Akteur ausgeübt wird, durch ein Beziehungsgeflecht aller weiteren Akteure untereinander ergänzt. Ein Beispiel für eine derartige Strukturform ist ein politisches Netzwerk auf kommunaler Ebene, in dem - vermittelt über gemeinsame Konferenzen, Gremienarbeit, Arbeitsgruppen etc. - die betroffenen Akteure in die Problemdebatte und -lösung einbezogen werden, sämtliche Steuerungs- und Entscheidungskompetenzen jedoch beim staatlichen Akteur verbleiben. Trotz der Dominanz eines Akteurs ist eine eigenständige Dynamik durch die Beziehungen der anderen Netzwerkbeteiligten untereinander gegeben.

4. Der letzte Netzwerktyp stellt das Ideal des völlig gleichberechtigten Miteinanders einer Gruppe von Akteuren dar, von denen sämtliche durch Beziehungen untereinander verbunden sind (heterarchisches Netzwerk). Dies könnte z. B. eine feste Arbeitsgruppe von Akteuren sein, die alle Aufgaben der Organisation und Steuerung reihum von Sitzung zu Sitzung rotieren lässt. Das Problem heterarchischer Netzwerke besteht darin, dass ein stabiles Steuerungssystem etabliert werden muss, das nicht auf Macht (wie in Hierarchien), sondern ausschließlich auf Verhandlung aufbaut. 
Dabei ist festzuhalten, dass es in den meisten Handlungsfeldern Sozialer Arbeit hierarchische wie heterarchische Netzwerke in „Reinform“ nur in Ausnahmefällen gibt. Die Akteure haben eine unterschiedlich starke Position auf der lokalen Ebene, aber eine einzelne fokale Organisation gibt es meist nicht - allerdings mehrere „starke Partnerinnen und Partner“ (z. B. Kommune, Grundsicherungsstelle), deren Einfluss auf die Beziehungen im Netzwerk genau beobachtet werden muss. Gerade wenn eine bilaterale Kooperation „starker Partnerinnen und Partner“ aus politischen oder ökonomischen Gründen erschwert ist, kann eine multilaterale Kooperation über das Hinzutreten „neutraler“ Akteure neue Wege der Zusammenarbeit bahnen. Allerdings ist umgekehrt auch eine nachhaltige Blockierung von Entwicklungen möglich, wenn „starke Beteiligte“ eine Koalition der Verhinderung bilden.

\subsubsection{Richtungsoffene und zielgerichtete Netzwerke}

In der Netzwerkforschung werden „richtungsoffene“ und „zielgerichtete Netzwerke“ unterschieden. Schubert schreibt hierzu: „Richtungsoffene Netzwerke haben den Charakter kohärenter korporativer Gemeinschaften, die nicht vertikal-hierarchisch strukturiert, sondern horizontal verbunden sind. Der dauerhafte Zusammenhalt wird durch Vertrauen untereinander gefestigt. Die Kohäsion bildet sich aber auch aus, weil die Akteure gemeinsame Grundüberzeugungen im Sinne eines strategisch ausgerichteten Leitbildes und in der Form von Leitwerten entwickeln" (Schubert 2008: S. 47). Demgegenüber verfolgen zielgerichtete Netzwerke klar definierte Aufgaben und Zwecke. „Die zu bewältigende Aufgabe ist zu komplex, als dass sie von einem Akteur allein zu bewältigen wäre. Die Akteure haben jeweils spezifische Eigenschaften, an denen andere interessiert sind, und initiieren beziehungsweise koordinieren Tauschprozesse untereinander" (ebd.).

Diese Unterscheidung wird im Folgenden aufgegriffen und im Hinblick auf funktionale Aspekte als Differenz von vier Netzwerktypen gefasst: Informationsnetzwerke, milieubildende Netzwerke, Projektnetzwerke und Produktionsnetzwerke.

- In „Informationsnetzwerken“ werden im Wesentlichen Informationen ausgetauscht, die für alle Netzwerkakteure von Relevanz sein könnten, aber von jeder beteiligten Organisation auch nach eigenen Relevanzkriterien bearbeitet werden. Der Grad an Verbindlichkeit mag sich unterscheiden, gemeinsam ist allen Informationsnetzwerken, dass die Ge- 
schäftsfelder der einzelnen Organisationen durch die Beteiligung am Netzwerk nicht oder nur minimal berührt werden.

- Dies trifft im Kern auch auf „milieubildende Netzwerke“ zu. Im Gegensatz zu reinen "Informationsnetzwerken“ wird aber hier ein gemeinsames „Thema“ formuliert, werden „politische Probleme definiert, Situationsdeutungen und politische Problemlösungen herausgebildet und politische Handlungsoptionen (...) mit dem Ziel einer kollektiven Entscheidungsfindung und Kompromissbildung zur gemeinsamen Nutzenmehrung" (Hild 1997: S. 216) selektiert. Diese Netzwerke können mehr oder minder stabil sein, sie heben sich aber durch die Dauerhaftigkeit des Beziehungszusammenhangs aus dem „organisationalen Feld" hervor, das die neoinstitutionalistische Theorie als relevante Umwelt von Organisationen konzipiert hat und im Falle von Unternehmen von Konkurrentinnen und Konkurrenten, Zulieferern, Kundinnen und Kunden, aber auch Regulationsbehörden, Gerichten etc. gebildet wird. Sie konstituieren unter Umständen ein „ideelles Milieu“ (vgl. Bauer 2005), das aus gemeinsam geteilten Wertorientierungen, Handlungskonzepten und Professionsvorstellungen gebildet wird. ${ }^{23} \mathrm{Da}$ bei Informationsnetzwerken und milieubildenden Netzwerken nicht die gemeinsame Erstellung von Produkten oder Dienstleistungen (vgl. Sydow/Möllering 2009: S. 15) im Vordergrund steht, stellt sich im Falle horizontaler Verknüpfungen das Problem der Konkurrenz weniger scharf - auch sind die Anforderungen an die Intensität von Kooperation nicht so hoch wie bei zielorientierten Netzwerken. Zielorientierte Netzwerke können als „Projektnetzwerke“ oder „Produktionsnetzwerke" beschrieben werden.

- Projektnetzwerke haben als „temporäre Netzwerke“ ihre Funktion darin, dass eine organisationsübergreifende Aufgabenstellung zeitlich befristet bearbeitet wird, z. B. im Rahmen eines „Projekts“. Bei „Projektnetzwerken“ handelt es sich um „eine Organisationsform ökonomischer Aktivitäten, die im Zusammenspiel projektbezogener und projektübergreifender Aktivitäten koordiniert wird" (Sydow/Wirth/ Manning 2002: S. 3). Dies bedeutet, dass für ein zeitlich befristetes Projekt auf einen Pool von autonomen Akteuren zurückgegriffen wird. Dabei werden Beziehungszusammenhänge aktualisiert, die sich z. B. im Rahmen eines Produktionsnetzwerks konstituiert haben, aber durchaus auch lockerer geknüpft sein können (Informationsnetzwerk). Die Er-

23 Gerade die mit dem Begriff „Bündnis“ bezeichneten Netzwerke zeichnen sich durch ein hohes Maß an geteilten Wertorientierungen aus. 
fahrungen aus früherer Zusammenarbeit, verbunden mit der Erwartung, bei künftigen Projekten (wieder) berücksichtigt zu werden, machen die Projektkoordination häufig erst möglich (vgl. ebd.: S. 4).

- Das Kennzeichen von „Produktionsnetzwerken“24 besteht demgegenüber darin, dass autonome Organisationen über einen zum Teil längeren Zeitraum gemeinsam Produkte herstellen und/oder Dienstleistungen erbringen. Es ist ersichtlich, dass hierzu ein intensiverer Kooperationszusammenhang erforderlich ist als in Politiknetzwerken. Allerdings ist es nicht notwendig, dass alle Akteure bei jedem Produktionsvorgang beteiligt sind - um von einem Produktionsnetzwerk zu sprechen, reicht es aus, dass alle Beteiligten verbindlich ihre Bereitschaft erklären, dann zu einem gemeinsamen Produkt beizutragen, wenn dies benötigt wird. Produktionsnetzwerke können entweder eine stärker hierarchische oder heterarchische Struktur aufweisen - zentral ist das Problem, über einen längeren Zeitraum Verbindlichkeit zu sichern.

„Projektnetzwerke“ und „Produktionsnetzwerke“ können direkt aufeinander bezogen sein (vgl. Wirth 2007). In dieser Konstellation existieren bereits Netzwerkstrukturen, die für die Bearbeitung einzelner Projekte jeweils aktiviert werden. Auf der "Systemebene“ wurden Vernetzungen geschaffen, die je nach Bedarf auf der „Projektebene“ abgerufen werden können - oder auch nicht. ${ }^{25}$ Dieser Gedanke lässt sich auch auf das Case Management anwenden. Wenn dieses eingebettet ist in ein „Produktionsnetzwerk", werden die Mitarbeitenden im Case Management ihrer alltäglichen Arbeit entlastet - gleichzeitig wird die Möglichkeit für die Adressatinnen und Adressaten von Produktionsnetzwerken geschaffen, diese Angebotsstrukturen auch ohne einzelfallbezogenes Case Management zu nutzen wenn sie dazu in der Lage sind. Allerdings sind ebenso temporäre Netzwerke ohne dahinterstehende Produktionsnetzwerke möglich - und häu-

24 Dieser Begriff darf nicht zu eng gefasst werden. Er bezeichnet die kontinuierliche Erstellung von materiellen Produkten und/oder Dienstleistungen in einem Netzwerk und bezieht sich nicht nur auf materielle Produktion. Sydow/Möllering setzen ihn inhaltlich gleich mit "Unternehmensnetzwerken“ (Sydow/Möllering 2009: S. 17) - ein Begriff, der für den sozialen Bereich nicht recht passt, da die hier vertretenen Organisationen nur teilweise Unternehmen sind.

25 Der Unterschied zwischen dieser Konstellation und einem reinen „Projektnetzwerk" besteht darin, dass die Akteure eines Produktionsnetzwerks nicht nur „latente" Beziehungen zueinander haben, die in einem Projekt evident werden, sondern dass stabile (d. h. verbindliche) Netzwerkbeziehungen existieren, auf die jeweils fallweise zurückgegriffen wird. Sie sind bereits evident, auch wenn sie nicht in allen Fällen benötigt werden. 
fig auch Realität. In diesem Fall müssen die zur Bearbeitung notwendigen Ressourcen immer wieder von Fall zu Fall oder von Projekt zu Projekt trägerübergreifend organisiert werden. Damit geht die „Systemebene“ direkt aus der Fallebene hervor - beides resultiert aus der Arbeit der Fall- oder Projektmanagern. Zum Teil stellen Netzwerke komplexe Gebilde dar, sind z. B. Bestandteil umfassender Netzwerkarchitekturen. Häufig sind Informationsnetzwerke (z. B. „lokale Bündnisse für Familie“) und Produktionsnetzwerke miteinander verknüpft - allerdings sollten sie nicht vermischt sein, denn der unterschiedliche Grad an geforderter Verbindlichkeit beinhaltet unterschiedliche Anforderungen an die Verlässlichkeit der Akteure des Netzwerks. Zudem stellt sich das Problem der Konkurrenz in Informationsnetzwerken weitaus weniger scharf als in Produktionsnetzwerken. Auch können Informationsnetzwerke und Produktionsnetzwerke auseinander hervorgehen und eng aufeinander bezogen sein, allerdings muss deren jeweilige Eigenlogik beachtet werden. Schubert spricht in Bezug auf sozialräumliche Netzwerke - in etwas anderer Terminologie - von „richtungsoffenen Vernetzungsfeldern" einer- und ziel- bzw. zweckgerichteten „Teilnetzwerken“ andererseits (vgl. Schubert 2005), die über eine Koordinationsebene miteinander verbunden werden. Es ist eine nur empirisch zu beantwortende Frage, ob und auf welche Weise sich aus einem Informationsnetzwerk ein Produktionsnetzwerk herausschält, denn für letzteres sind - wie gezeigt - engere Beziehungszusammenhänge notwendig. Ein „ideelles Milieu“, das sich innerhalb eines Informationsnetzwerks konstituiert hat, bildet zwar den idealen Humus für die Konstitution eines Produktionsnetzwerks, gleichzeitig können aber Macht- oder Konkurrenzverhältnisse, die in Produktionsnetzwerken deutlicher zum Vorschein kommen, den Konsens in Informationsnetzwerken untergraben. Damit offenbart sich die Bedeutung der internen Prozesse und Beziehungen in Netzwerken.

Die Ausdifferenzierung dieser Netzwerktypen kann - gerade in Kombination mit der Unterscheidung verschiedener Konstellationen von Kooperation (vgl. 4.2.1) - die Entscheidung erleichtern, welcher Typ für welches Vorhaben geeignet ist, d. h. welchen Grad an Verbindlichkeit die Akteure des Netzwerks einzugehen bereit sind.

\subsection{Theoretische Zugänge zum Verständnis von Netzwerken}

Die Unterscheidung von Netzwerktypen ist sinnvoll, um einen ersten Überblick über das schillernde Feld von Netzwerkbildungen zu erhalten. 
Sie reicht aber nicht aus, um empirische Entwicklungen zu erklären und ist auch nicht hinreichend konkret, um Anregungen für die Praxis der Netzwerkbildung und -stabilisierung zu geben.

Deshalb sollen in diesem Kapitel verschiedene theoretische Zugänge zum Thema Netzwerkbildung dargestellt werden, die auf unterschiedliche Aspekte fokussieren:

- Die Besonderheiten von Netzwerken als Form von Governance sowie die Mechanismen dieser Governance werden über einen strukturtheoretischen Zugang erschlossen - der Leitbegriff ist hier "Struktur“ (4.3.1).

- Die Frage, was Netzwerke „zusammenhält“, wird über einen sozialpsychologischen Zugang mit dem Leitbegriff „Kultur“ beantwortet (4.3.2).

- Produktionsnetzwerke erfordern engere Kooperation zwischen den Beteiligten. Welche Probleme sich hier stellen und wie „vernetzte“ Dienstleistungen effektiv erarbeitet werden können, sind Themen eines dienstleistungstheoretischen Zugangs - der Leitbegriff ist hier „Prozess" (4.3.3).

\subsubsection{Ein strukturtheoretischer Zugang zu Netzwerken}

Ein großer Teil der Literatur zu organisationalen Netzwerken beschäftigt sich mit Fragen der Struktur dieser Netzwerke, ihrer Stabilität und der Modi ihrer Steuerung. Referenzmodell ist dabei häufig das der „Organisation“ mit dem Steuerungsmodus der „Hierarchie“. Payer hat die zentralen Unterschiede der drei analytisch getrennten Governance-Typen Hierarchie, Markt und Netzwerk folgendermaßen systematisiert (vgl. Payer 2002: S. 27):

- Märkte haben ihre normative Basis in Eigentumsrechten (Verträge) und werden über das Medium „Geld“ gesteuert.

- Hierarchie hat ihre normative Basis in Weisungsrechten und werden über das Medium „Macht“ gesteuert.

- Netzwerke haben ihre normative Basis in Vertrauen und Verlässlichkeit und werden über das Medium „Wissen“ gesteuert.

Da diese Netzwerke von Organisationen gebildet werden, müssen sich die im Netzwerk handelnden Personen mit dem Problem des „doppelten Bezugsrahmens" auseinandersetzen, ihrer Bindung an die Herkunftsorganisation bei gleichzeitiger Loyalität gegenüber dem Netzwerk. Dieses Problem resultiert aus der grundlegenden Autonomie der Netzwerkmitglieder (4.3.1.1). Die Autonomie wiederum setzt hierarchiegeprägten Steuerungs- 
instrumenten wie Weisungen klare Grenzen und legt „weiche“ Steuerungsinstrumente wie Vertrauen nahe (4.3.1.2).

\subsubsection{Die Grundproblematik von organisationalen Netzwerken}

Die Herausbildung und Stabilisierung sozialer bzw. ökonomischer Beziehungen zwischen autonomen Akteuren und die damit verbundene Grundproblematik eines „organisationalen Netzwerks“ wird in der Literatur häufig im Rückgriff auf die Systemtheorie präzisiert (vgl. z. B. Schubert 2008; Großmann/Lobnig/Scala 2007).

Organisationale Netzwerke sind mit einem dreifachen Integrationsproblem konfrontiert:

- Das „Binnenproblem“, „bei dem interne Fragen der Artikulation, Aggregierung und Selektion von systemischen Mitgliederinteressen, der Definition von Systemzwängen, der Bildung einer Binnenmoral, der Abstimmung intern ausdifferenzierter Rollen und die Frage der inneren Verteilung von Ressourcen im Blickpunkt stehen“ (Schubert 2008: S.30). Hier geht es um die internen Relationen des Systems „Netzwerk“, letztlich um den Aufbau des Netzwerks als eigenständiger kollektiver Akteur.

- Das „Außenproblem“ des Netzwerks, das sich in einer Systemumwelt positionieren muss, die von anderen Systemen (z. B. auch anderen Netzwerken, netzwerkexternen Organisationen etc.) und vom umfassenden Gesamtbezugssystem gebildet wird (z. B. einem bestimmten Politikfeld oder auch „der Gesellschaft“). Über seine Positionierung gegenüber dieser Umwelt gewinnt das Netzwerk Identität, dabei darf aber nicht vergessen werden, dass die meisten der am Netzwerk beteiligten Organisationen ein analoges „Außenproblem“ haben, d. h. sich ihrerseits positionieren müssen), was zu Interessensdivergenzen führen kann.

- Das „Grenzproblem“, das z. B. an dieser Stelle ansetzt und mit der Abstimmung divergierender Ziele zu tun hat. Jede beteiligte Organisation hat eigene Interessen, die in die Arbeit im Netzwerk einfließen. „Im Kreis der vernetzten organisationalen Systeme bilden sich Erwartungen (in Form von Interessen, Zielen, Rationalitätskriterien) heraus, die untereinander abgestimmt werden müssen“ (ebd.: S. 31).

Der Begriff der „doppelten Grenze“ formuliert dieses Problem als spezifisches Spannungsfeld. Er „thematisiert, dass es neben der Grenze, die Netzwerksystem und Netzwerkumwelt im Sinne von ,Netzwerk-Außenwelt 
trennt, auch eine Grenze des Systems gegenüber seinen Mitgliedern als ,Netzwerk-Innenwelt' gibt. Denn im Rahmen der Vernetzung kann ein organisationales System einerseits im Innenverhältnis dazugehören und andererseits in seinen äußeren Beziehungen außerhalb des Netzwerksystems stehen. Vor diesem Hintergrund steht ein Netzwerk als System zwei verschiedenen Umwelten gegenüber und muss daher Abstimmungsprobleme zwischen Innenwelt und Außenwelt lösen“ (ebd.). Das Problem der „doppelten Grenze“ wird unmittelbar handlungsrelevant über die Personen, die als Vertretungen von Organisationen im Netzwerk tätig sind. Sie handeln in einem „doppelten Bezugsrahmen“: Dem des Netzwerks sowie dem der einzelnen Organisation und damit gleichzeitig als (zumeist angestellte) Angehörige einer „Mutterorganisation“ (Kommune/Agentur für Arbeit/ Träger) und als Mitglied des Netzwerks. Hirsch-Kreinsen formuliert das damit verbundene Grundproblem: „So müssen die Manager der einzelnen Netzwerkunternehmen die Interessen des jeweils eigenen Unternehmens mit den Zielen und Erfordernissen des Unternehmensnetzwerks insgesamt abstimmen, wobei nicht immer eindeutig zu bestimmen ist, welchen Interessen dabei Priorität zukommen sollte" (Hirsch-Kreinsen 2002: S. 115). Van Santen/Seckinger haben hieran die Problematik der „doppelten Adäquanz" und der "doppelten Zielkongruenz" festgemacht, wenn ein personaler Akteur die Handlungen zwischen der Loyalität zur „Herkunftsorganisation" und der Loyalität zum Netzwerk austarieren muss (vgl. van Santen/Seckinger 2003; van Santen/Seckinger 2005). Der „doppelte Bezugsrahmen" der im Netzwerk handelnden Personen ist nach Hild verantwortlich für Blockaden, die sich wegen Verteilungskonflikten und/oder Interessensgegensätzen in Netzwerken ergeben können (vgl. Hild 1997: S. 220). Aus diesem Grund ist es auch nicht sinnvoll, einem Netzwerk per se den Status eines „kollektiven Akteurs" zuzuschreiben, vielmehr muss kollektive Handlungsfähigkeit „erst gemeinsam (...) produziert und aufrechterhalten werden" (Windeler 2001: S. 244) - was den prozessualen Charakter von Netzwerken nochmals betont.

\subsubsection{Vertrauen, Verlässlichkeit und Reziprozität}

Produktionsnetzwerke sind - ähnlich wie Organisationen - zielorientiert. Sie unterscheiden sich von formalen Organisationen dadurch, dass eine einheitliche Leitung fehlt (vgl. Windeler 2001), was Konsequenzen für die Einsatzmöglichkeiten hierarchisch ausgerichteter Steuerungsinstrumente wie z. B. Weisungen hat. Sie können aber im Hinblick auf die Steuerungs- 
problematik analog zu Organisationen betrachtet werden. Aus diesem Grund kann eine Systematisierung der Steuerung von Dienstleistungsproduktion in Organisationen mit leichten Modifikationen auch auf die Steuerung von Produktionsnetzwerken angewandt werden.

Die Steuerung von Organisationen kann im Anschluss an Niklas Luhmann (vgl. Luhmann 2000) analog zum Verhältnis von faktischer Entscheidung (operative Handlung) und Festlegung von Entscheidungsprämissen (strategische Setzung eines Rahmens für operative Handlungen) konzipiert werden. Luhmann differenziert dabei zwischen unentscheidbaren und entscheidbaren (durch die Organisationsspitze strategisch setzbaren und strategisch nicht setzbaren) Entscheidungsprämissen (vgl. ebd.). Vor allem Letztere sind interessant, denn sie können Gegenstand bewusster Steuerung sein, während die „Organisationskultur“ als unentscheidbare Entscheidungsprämisse nur indirekt beeinflusst werden kann. Innerorganisatorisch „entscheidbar" sind nach Luhmanns Auffassung

- Entscheidungsprogramme, d. h. verbindliche Regeln, wie in einer Organisation entschieden werden soll.

- Kommunikationswege, die eingehalten werden müssen, wenn eine Entscheidung als Organisationsentscheidung gelten soll.

- Personaleinsatz, d. h. der Einsatz eines in bestimmter Weise qualifizierten und kompetenten Personals.

Den drei Typen von Entscheidungsprämissen entsprechen die „Steuerungsbereiche" Regeln, Strukturen und Personen. Diese stecken in ihrer konkreten Ausgestaltung den Raum der Handlungsmöglichkeiten innerhalb einer Organisation ab. „Steuerung“ bedeutet somit, Regeln, Strukturen und Personen zu entwickeln und zu implementieren (um damit strategisch Entscheidungsprämissen zu setzen) und den Erfolg oder Misserfolg der Steuerung an den Resultaten der operativen Handlungen zu messen (mit Rückkopplung auf die Reflexion der gesetzten Entscheidungsprämissen). Mit diesen drei Steuerungsbereichen ist das Problem der Steuerung komplexer Prozesse aber nicht hinreichend gefasst. Wie die systemtheoretisch ausgerichtete Organisationstheorie gezeigt hat, sind dort, wo die Erreichung von Organisationszielen davon abhängt, dass Personen oder andere Organisationen autonom, aber dennoch „gesteuert“ handeln, „weiche“ Steuerungsimpulse gefragt, die unter dem Begriff der „Kontextsteuerung" diskutiert werden (vgl. Naujoks 1994; Breisig 2010). Dies bedeutet im Kern, dass Entscheidungen in Organisationen indirekt beeinflusst werden, z. B. durch die Weitergabe von Informationen, das Setzen von Anreizen $u$. ä. 
Wie die Kontextsteuerung zeigt, sind auch in Organisationen immer dort, wo die Erreichung von Organisationszielen davon abhängt, dass Personen oder andere Organisationen autonom, aber dennoch "gesteuert“ handeln, „weiche“ Steuerungsimpulse gefragt. Dies gilt erst recht für die Netzwerksteuerung, denn hier geht es um autonome Akteure, die über die genannten Entscheidungsprämissen nur begrenzt steuerbar sind. Zwar spielt die Festlegung von Regeln und Kommunikationswegen auch in Netzwerken eine Rolle (vgl. 4.6.4.1), im Unterschied zu Organisationen kann deren Geltung aber nicht mit hierarchischen Mitteln durchgesetzt werden. Vielmehr sind Netzwerke zur Stabilisierung ihrer Strukturen im Hinblick auf Regeln, Kommunikationswege und Personal auf andere „weiche" Steuerungsmodi angewiesen. Als typische Steuerungsmedien, die zur Konfliktbewältigung und Stabilisierung in Netzwerken eingesetzt werden, führen Sydow/Windeler im Rekurs auf einschlägige Literatur auf (vgl. Sydow/Windeler 2000: S. 12ff.):

- Kooperation,

- Vertrauen,

- Selbstverpflichtung der Akteure,

- Verlässlichkeit,

- Verhandlung,

- explizite oder implizite Kontrakte.

Das gemeinsame Merkmal dieser Steuerungsmedien ist, dass sie teilweise nur schwer formalisierbar sind.

Fast in der gesamten einschlägigen Literatur findet sich der Hinweis auf die grundlegende Bedeutung von „Vertrauen“ als Basis der Netzwerkkommmunikation und Netzwerksteuerung. So stellen Lindsay et al. in einer vergleichenden Fallstudie über Kooperation in der lokalen Arbeitsmarktpolitik in Großbritannien fest: „Strong lines of trust and reciprocity, rather than the demands of contract agreements, characterized relationships between lead partners and other participating organizations" (Lindsay/McQuaid/Dutton 2008: S. 729; Hervorhebungen im Original). Allerdings ist „Vertrauen (...) kein Naturphänomen oder Schicksal, sondern eine kommunikative Erfahrung" (Großmann/Lobnig/Scala 2007: S. 136), die an die interpersonalen Beziehungen zwischen den Beteiligten gebunden ist. Zu einer kommunikativen Erfahrung kann Kooperation vor allem dort werden, wo an einem gemeinsamen „Produkt“ gearbeitet wird. Denn in der praktischen Auseinandersetzung mit konkreten Aufgaben können die Beteiligten Sichtweisen und Erfahrungen austauschen und aufeinander beziehen (vgl. ebd.: S. 117). Das Problem von Vertrauen als zentraler Basis für den Aufbau von Kooperationen besteht darin, dass es häufig zu Beginn 
einer Kooperationsbeziehung nicht gegeben ist. Vielmehr lebt „Vertrauensbildung" von Vertrauensvorschüssen, d. h. Erwartungen darüber, was die Zusammenarbeit bringen kann, und was die einzelnen Akteure dazu beitragen werden. „Trust is the anticipation that something will be forthcoming in return for the efforts that are put into the collaboration" (Huxham/Vangen 2005: S. 154). Allerdings beinhaltet jeder Vertrauensvorschuss das Risiko der Enttäuschung von Erwartungen.

Eine wichtige Aufgabe für Netzwerke ist deshalb der Aufbau und die Stabilisierung von Vertrauensbeziehungen, etwa durch häufige Kommunikation und den laufenden Austausch von Informationen. Dies kann unter Umständen ein langwieriger Prozess sein, wie Huxham und Vangen hervorheben: „Undoubtedly, the trust building loop aligns itself well with a ,small wins' approach (...) within which trust can be built through mutual experience of advantage gained via successful implementation of low-risk initiatives" (ebd.: S. 160). Vertrauensbildung ist mithin ein zyklischer Prozess, in dessen Verlauf erste Ergebnisse und Erfolge dazu beitragen, Vertrauen zu festigen und auszubauen. Auf dieser Basis können dann neue Aufgaben angegangen werden. Vertrauensbildung ist also ein sich selbst bestätigender Prozess, der voraussetzt, „dass die Kooperationspartner über eine klare Vorstellung der Aufgaben und des Angebotsprofils der jeweils anderen verfügen“" (van Santen/Seckinger 2003: S. 347). Großmann, Lobnig und Scala heben den gleichen Aspekt hervor: „Die handelnden Personen sind vor allem herausgefordert, sich mit den unterschiedlichen Traditionen, Arbeitsweisen, kulturellen Eigenheiten, identitätsstiftenden Leistungen und Restriktionen der potenziellen Partner auseinander zu setzen“ (Großmann/Lobnig/Scala 2007: S. 55). Der Prozess der Vertrauensbildung wird erschwert, wenn Netzwerkpartnerinnen und -partnergleichzeitig Konkurrenz sind. In lateralen Netzwerken kooperieren Organisationen, die auf der gleichen Stufe der Dienstleistungskette operieren - damit stellt sich dieses Problem rasch. So kooperieren in Netzwerken im Bereich der Sozial- und Arbeitsmarktpolitik Beschäftigungs- und Bildungsträger, aber auch örtliche Gliederungen von Verbänden der freien Wohlfahrtspflege, obwohl sie z. B. im Kontext von Vergabeverfahren gleichzeitig im Wettbewerb stehen. Da im Zuge der sozialpolitischen Entwicklung der letzten Jahre auch in Deutschland der Wettbewerbsgedanke immer stärker um sich greift, spielt dieses Thema auch im Sozialbereich eine Rolle. Wichtig ist zunächst, dass es weniger auf eine „objektive“ Konkurrenz ankommt, sondern darauf, wie die Akteure diese wahrnehmen und bewerten. Unter einer sozialkognitiven Perspektive ist von Konkurrenz die Rede, wenn sich ein Unternehmen an einem anderen orientiert und die Merkmale und 
Handlungen des anderen Unternehmens in seinen Entscheidungen mit dem Ziel berücksichtigt, aus seinen Erkenntnissen Wettbewerbsvorteile zu erzielen (vgl. Lerch/Sydow/Wilhelm 2007: S. 212). Es kommt somit auf die Wahrnehmung und das faktische Handeln, weniger auf die objektive Konstellation an. Damit ist aber die grundsätzliche Möglichkeit gegeben, dass auch „Konkurrenten“ gemeinsam an der „kognitiven Domestizierung“ (ebd.: S. 244) des Wettbewerbs arbeiten. Entschärfend wirkt z. B. die Norm des "fairen Wettbewerbs“ (ebd.: S. 241), die Kooperation solange zulässt, wie niemand die Ergebnisse der Kooperation ausnutzt, um sich einseitig Wettbewerbsvorteile zu verschaffen. Hier spielen Vorerfahrungen der Akteure untereinander und miteinander eine große Rolle.

Organisationen beteiligen sich trotz potenzieller Konkurrenz an horizontalen Netzwerken, weil sie sich Vorteile versprechen, die dieses Manko aufwiegen:

„1. Die Realisierung interner und externer Skalenerträge durch bessere Auslastung der Kapazitäten und Ermöglichung der Anschaffung sonst wenig ausgelasteter Spezialmaschinen.

2. Die Möglichkeiten zur Kombination unterschiedlicher Kompetenzen im Produktionsbereich.

3. Die Freisetzung von Kapital für andere wirtschaftliche Aktivitäten"

(Sydow/Möllering 2009: S. 217).

Hinzu kommt die Gewinnung von zusätzlichem Know-how, das auf andere Geschäftsfelder übertragen werden kann, in denen sich die Akteure des Netzwerks nicht als Konkurrenz gegenübertreten.

Vertrauen entsteht durch die Erfahrung von „Verlässlichkeit“: „Während Vertrauen (...) nur ein Sonderfall von Verlässlichkeit ist, ist Selbstverpflichtung eine Möglichkeit unter mehreren - wie der Steigerung der informellen Transparenz oder der Verdeutlichung möglicher Sanktionen die Verlässlichkeit zu steigern" (Sydow/Windeler 2000: S. 14). Zum selben Schluss kommen auch Fürst und Schubert: „Dabei bilden positive emotionale Zuschreibungen und verlässliche Beziehungen die wesentlichen Vorrausetzungen der internen Kohäsion" (Fürst/Schubert 1998: S. 352). Verlässlichkeit drückt sich aus in der mittelfristigen Reziprozität der Beziehungen: „Hierunter wird ein Prinzip des Leistungsaustauschs verstanden, das nicht durch die wertmäßige Äquivalenz der einzelnen Transaktion (wie beim marktförmigen Austausch), sondern durch die in der Summe ungefähre Äquivalenz einer Reihe von Austauschen über einen längeren Zeitraum hinweg gekennzeichnet ist. Er setzt die Existenz langfristiger Beziehungen zwischen Akteuren somit voraus und stabilisiert sie zugleich“ 
(Kohlmeyer/Mauruszat/Seyfried 2000: S. 10). Die verlässlichen Beziehungen sind zugleich Medium und Resultat von Macht und Herrschaft (vgl. Sydow/Windeler 2000: S. 16). Denn jede Stabilisierung von Netzwerken hängt $\mathrm{ab}$ von sozialen Praktiken, deren Ausprägung mit dem konkreten Problembezug und der Konstellation der Akteure zueinander korrespondiert: „Es existiert (...) keine idealtypische innere Strukturform (Aufbauund Ablauforganisation), die grundsätzlich und für alle Netzwerke ein reibungsloses Funktionieren gewährleistet. Im Gegenteil: Netzwerke müssen ihre Struktur an ihrer Akteurskonstellation wie auch ihren Problemfeldern oder Aufgabenstellungen orientieren und somit an ihre spezifische Situation angepasste innere Strukturen jeweils entwickeln" (Kohlmeyer/Mauruszat/Seyfried 2000: S. 15). „Vertrauen“ und „Reziprozität" rücken in den Status von Entscheidungsprämissen: Künftige Entscheidungen hängen davon ab, welche Erfahrungen die Akteure des Netzwerks mit Vertrauensvorschüssen und mit der - mittelfristigen - Reziprozität von Entscheidungen in der Vergangenheit gemacht haben. Für die Bildung von "Vertrauen“ und die Realisierung von Reziprozität ist es förderlich, dass ein „ideelles Milieu" existiert, d. h. ein gemeinsam geteilter Ziel- oder Wertkorridor, der die Basis bildet für die Bewertung inhaltlicher „Verlässlichkeit“ (formal macht sich Verlässlichkeit an der Befolgung gesetzter Regeln etc. fest dies reicht aber nicht, um ein Netzwerk zu stabilisieren). Deshalb sind auch und gerade Produktionsnetzwerke darauf verwiesen, dass ein ideelles Milieu entsteht und gepflegt wird. Gemeinsam entwickelte Ziele und ein Konsens oder zumindest Kompromiss im Hinblick auf grundlegende Werte und Überzeugungen bilden die Basis für die Konstruktion von Leistungsketten, die über Koppelproduktion dazu führen, dass diese Ziele auch realisiert werden.

\subsubsection{Ein sozialpsychologischer Zugang zu Netzwerken}

„Vertrauen“ und „Reziprozität“ werden - wie gesehen - in der Literatur als "Strukturelemente" von Netzwerken betrachtet, ohne deren Vorhandensein Netzwerke - wenn sie überhaupt zu Stande kommen - schnell instabil werden. Nur selten wird die Frage systematisch behandelt, auf welchen Mechanismen Vertrauensbildung basiert. Mit dieser Frage haben sich bereits auseinandergesetzt und gezeigt, dass die Entwicklung interorganisationaler Beziehungen (und damit auch von organisationalen Netzwerken) als ein Prozess anzusehen ist, in dem sich formale Elemente (z. B. Regeln, Vereinbarungen) und informelle Beziehungen miteinander verschränken. 
Bereits „Reziprozität“ kann nicht als formales ökonomisches Kalkül verstanden werden, sondern ist eingebettet in ein kulturell geprägtes "Gefühl“ von Fairness („fair dealing“). (vgl. Ring/Van de Ven 1994: S. 93f.) Um zu verstehen, wie Kooperationen funktionieren, muss die Frage beantwortet werden, „how people can make present commitments to engage in uncertain future courses of action and the sources of security that lead them to believe that the terms of their representations and commitments are congruent and will be enforceable“ (Ring/Van den Ven 1994: S. 99).

Ring und Van de Ven versuchen eine Antwort, indem sie zeigen, dass Kooperationsstrukturen durch die Verständigung der beteiligten Personen (d. h. personaler Akteure als Repräsentantinnen und Repräsentanten von Organisationen) über gemeinsame Sichtweisen auf die Kooperation und die gegenseitigen Interessenslagen entstehen (d. h. ein Prozess der gemeinsamen Sinnstiftung) und sich hierüber ein „psychologischer Kontrakt“ herausbildet (vgl. ebd.: S. 100). Dieser baut auf den konkreten Erfahrungen mit Kooperation auf, ist somit Resultat eines inkrementalen Prozesses. Entscheidend ist dabei, dass eine ursprünglich formale Kooperation normativ „aufgeladen“ wird und damit eine psychologische Bindungswirkung entsteht: „The institutionalization of a relationship is evident in three basic interactions that evolve over time between formal and informal processes of negotiation, commitment, and execution: (a) personal relationships increasingly supplement formal role relationships, (b) psychological contracts increasingly substitute for formal legal contracts, and (c) as the temporal duration of relationships extend beyond the tenure of initial contracting agents, formal agreements (e. g., rules, policy, contracts) increasingly mirror informal understandings and commitments. These interactions between formal and informal characteristics of relationships are an innocuous product of the social-psychological dynamics of interpersonal socialization and norm formation" (ebd.: S. 103). Sind diese Bindungen hergestellt, können persönliche Beziehungen organisationale Beziehungen überlagern, ohne sie aber zu ersetzen. Allerdings sind formale Regelungen zur Sicherung von Kontinuität sinnvoll: „When the temporal duration of interorganizational relationships is expected to exceed the tenure of agents, informal understandings and commitments will be formalized" (ebd.: S. 106). Somit ist die Balance, die gegenseitige Ergänzung von formalen und informalen Strukturen und Beziehungen entscheidend für den Prozess der Entwicklung und Stabilisierung interorganisationaler Strukturen. 


\subsubsection{Ein dienstleistungstheoretischer Zugang zu Netzwerken}

Die strukturtheoretisch argumentierenden Autorinnen und Autoren benennen einhellig die Existenz gemeinsam geteilter Ziele als einen wichtigen Faktor für den Aufbau und die Stabilisierung von Netzwerken. Dieses formale Argument verweist gleichzeitig auf die dahinter liegenden Inhalte, d. h. die „Substanz“ der Ziele. Vertrauensbeziehungen können genauso wenig wie Reziprozität von den Inhalten abgelöst werden, auf die sich die Kooperation im Netzwerk bezieht. Ein großer Teil der im Rahmen dieser Expertise verarbeiteten Literatur bleibt jedoch an dieser Stelle abstrakt und beschreibt die Herausbildung von Netzwerkstrukturen weitgehend unabhängig von den jeweiligen Inhalten. Insbesondere die Besonderheiten der Produktion sozialer Dienstleistungen werden nicht systematisch thematisiert.

Einen dezidiert anderen Weg schlägt das Forschungsteam des „Institute for Research on Poverty" der Universität von Wisconsin ein. In den Jahren 2004 bis 2008 entwickelten Thomas Corbett und Jennifer Noyes im Rahmen eines Forschungs- und Entwicklungsprojekts ein Modell für die $\mathrm{Zu}-$ sammenführung unterschiedlicher Dienste zur Unterstützung von Empfängerinnen und Empfängern der Leistungen der US-amerikanischen Sozialhilfe (das Projekt SINET - Service Integration Network, getragen von einem Netzwerk von Forschungs- und Entwicklungsinstituten sowie Regierungsbehörden, vgl. Corbett/Noyes 2004). Diese Zusammenführung war ein wichtiger Bestandteil der grundlegenden Reform des US-amerikanischen Fürsorgesystems im Jahr 1996. Corbett/Noyes thematisierten die im Zuge der Reform entstandenen Kooperationsprobleme zwischen den Institutionen des Fürsorge- bzw. Arbeitsmarktsystems. Sie identifizierten das jeweilige „institutionelle Milieu“ als den zentralen Faktor, der den Möglichkeitsraum von Kooperationen bestimmt. Das „Milieu“ einer Institution oder Organisation ist ein zusammenfassender Begriff für deren zugrundeliegende Normen, Werte und Verhaltensmuster, die die Art und Weise kennzeichnen, in der die Organisation funktioniert und Entscheidungen trifft. Welche Menschen werden für Schlüsselpositionen angeworben? Wie werden sie ausgebildet? Wie viel Freiheit wird den Mitarbeitenden und leitenden Angestellten darin gelassen, Entscheidungen zu treffen und nach eigenem Ermessen zu handeln? Welche Handlungsweisen der Mitarbeitenden werden erkannt und honoriert? Wie klar werden organisatorische Ziele und Werte artikuliert und wie bereitwillig werden diese von der Belegschaft akzeptiert? Dies sind nur ein paar Punkte in einer sehr langen Liste von Fragestellungen, die sich aus diesem Ansatz ergeben. Ein 
zentrales Element eines institutionellen Milieus ist seine „Kerntechnologie“ - die Tätigkeiten, die ausgeführt werden. Erbringt eine Organisation bspw. vorwiegend materielle Leistungen, bietet sie standardisierte Dienstleistungen an oder interveniert sie in Familien, um Probleme zu lösen oder Verhaltensmuster zu ändern? Diese fundamental unterschiedlichen Aufgabentypen bilden verschiedene Arten organisatorischer Milieus aus (vgl. Corbett/Noyes 2005: S. 28).

Corbett/Noyes rekurrieren in ihrer Analyse dabei auf Überlegungen, die zuerst von Michael Lipsky formuliert und später insbesondere in der anglo-amerikanischen Literatur breit aufgegriffen wurden (vgl. Lipsky 1980). Danach bringen es die Charakteristika personenbezogener Dienstleistungen mit sich, dass die Fachkräfte der Dienstleistungsorganisationen ein hohes $\mathrm{Ma}$ an sozialer Kompetenz benötigen, um ein „Arbeitsbündnis“ mit den Benutzenden aufbauen und aufrechterhalten zu können. Gleichzeitig ist für die Leistungserbringung eine hohe Flexibilität erforderlich, die selbst in ausgeprägt hierarchischen Organisationen Handlungsspielräume sichert. Diese Konstellation wurde von Lipsky als „Street-level bureaucracy" bezeichnet und analysiert - eine durch teilweise intensive Interaktion mit den Leistungsadressatinnen und -adressaten geprägte öffentliche Verwaltung. Lipsky fasst mit diesem Begriff Organisationen, die eine spezifische Art von Dienstleistungen erbringen, nämlich solche, die gesellschaftlich und politisch bedeutsam sind und deshalb vom Staat - meist auf gesetzlicher Grundlage - bereitgestellt werden. Er sieht ein mehr oder minder offenes Spannungsverhältnis zwischen der (für die Realisierung der Dienstleistung notwendigen) Handlungsautonomie einer- und der Steuerungsnotwendigkeit in Organisationen andererseits (vgl. Scott 2003; Hasenfeld 2010).

Yeheskel Hasenfeld greift diesen Gedanken auf und modifiziert ihn gleichzeitig durch die Unterscheidung verschiedener „Dienstleistungstechnologien“. Eine Dienstleistungstechnologie kann definiert werden als ein „Set von institutionalisierten Interaktionen, die darauf abzielen, die physischen, psychologischen, sozialen und kulturellen Eigenschaften von Personen zu verändern“ (Hasenfeld 1983: S. 111). Damit hebt Hasenfeld hervor, dass es in jeder Dienstleistungsorganisation weitgehend standardisierte und häufig „unhinterfragte“ Abläufe gibt, die das individuelle Handeln der Beteiligten „anleiten“, z. B. Programme, aber auch Rituale, Spiele etc. Die von Lipsky betonte Handlungsautonomie der Beschäftigten (vgl. Lipsky 1980) wird somit eingeschränkt, da sie eingebunden ist in routinisierte, habitualisierte Prozeduren mit unterschiedlichem Charakter. Hasenfeld unterscheidet zwischen 
- „People sustaining technologies“, d. h. Dienstleistungen, die darauf abzielen, die materielle bzw. physische Versorgung von Personen zu sichern. Hierzu zählt z. B. die Leistungssachbearbeitung im SGB II und SGB III - unabhängig davon, wie sie konkret organisiert ist.

- „People changing technologies“, d. h. Dienstleistungen, die dadurch charakterisiert sind, dass sie den ,inneren Zustand“ von Personen, also ihre Psyche, ihren Bildungsstand oder ihre Werthaltungen, verändern. Diesen Leistungen ist somit eine im weiteren Sinne therapeutische oder pädagogische Programmatik unterlegt.

- „People processing technologies“, d. h. Dienstleistungen, die den sozialen Status von Personen insofern verändern, als sie ihnen (neue) $\mathrm{Zu}$ gänge zu gesellschaftlichen Institutionen (z. B. dem Arbeitsmarkt) eröffnen. Sie arbeiten also - wie z. B. Arbeitsvermittlerinnen und -vermittler - am „Matching“ zwischen ihrer Kundschaft und gesellschaftlichen Ansprüchen (z. B. Ansprüchen der Arbeitgeberschaft).

Die Art der jeweiligen „Technologie“ der Leistungsprozesse hat Konsequenzen für die Beziehung der Akteure untereinander. Während nämlich Leistungen des „sustaining“ über klassische Konditionalprogramme standardisiert abgearbeitet werden können, indem Leistungsvoraussetzungen geprüft und je nach Prüfungsergebnis Leistungen in standardisierbarer Form gewährt werden (oder eben nicht), setzen Prozesse des "changing“ (und in eingeschränktem Maße auch solche des „processing“) erhebliche Autonomiespielräume auf der operativen Ebene voraus. Die Anwendung der Technologie kann jedoch nicht als formal-rationaler Prozess konzipiert werden, vielmehr setzt sie gesellschaftliche „Konventionen“ sowie politische und institutionelle Problemdefinitionen ebenso voraus wie das konkrete Handeln der involvierten Fachkräfte. Hasenfeld berücksichtigt dies mit seinem Konzept des praktischen institutionellen Wissens, der „Praxisideologie“ (practical ideology). Die Umsetzung dieses Wissens in Praxis geschieht im Kontext unterschiedlichen „Professionswissens“, welches das konkrete Handeln der Beschäftigten einer Organisation anleitet (vgl. Sandfort 2010).

Corbett/Noyes greifen die Idee unterschiedlicher „Dienstleistungstechnologien" auf, vereinfachen aber die Umsetzung im Hinblick auf Kooperationsstrukturen. Sie entwerfen eine Matrix zur Identifikation unterschiedlicher „Schwierigkeitsgrade“ der Kooperation bzw. Netzwerkbildung unterschiedlicher Organisationen. Die beiden Achsen der Matrix werden gebildet von der „institutionellen Ähnlichkeit“ der beteiligten Organisationen einer- und dem Grad der erforderlichen Kooperation (bis hin zu einer Integration) andererseits. Die „institutionelle Ähnlichkeit“ wird definiert 
am Grad der Routinisierung der Leistungserbringung. Hierbei unterscheiden Corbett und Noyes:

- Routinisierte Programme bzw. Organisationen. Dort erfolgt die Arbeit in hohem Maße nach vorgegebenen Regeln bzw. Standards in teilweise stark hierarchischen Organisationsstrukturen. Ein Beispiel sind (amerikanische) Sozialämter.

- Nicht-routinisierte Programme bzw. Organisationen. Die Arbeit folgt dort eher nach professionellen als nach bürokratischen Regeln, ist stark individualisiert, mit teilweise hohen Entscheidungskompetenzen des Personals und in meist flachen Hierarchien. Ein Beispiel ist Sozialarbeit bei freien Trägern.

- „Gemischte“ Programme bzw. Organisationen, in denen Arbeiten der beiden vorgenannten Typen parallel (aber meist von verschiedenen Berufsgruppen) verrichtet werden. Ein Beispiel sind Ämter, in denen materielle Leistungen und Eingliederungsleistungen für einen identischen Adressatenkreis angeboten werden (vgl. Corbett/Noyes 2008: S. 5).

Je nachdem, welche dieser organisationskulturellen Typen miteinander kooperieren sollen, stellen sich graduell unterschiedliche Probleme.

Corbett/Noyes argumentieren, dass bei allen Kooperationsvorhaben unabhängig vom Grad der Kooperation ${ }^{26}$ die gesamte Organisation in ihrem „institutionellen Milieu“ im Blick behalten werden muss. Dies gilt umso mehr, je intensiver die Kooperation ist. Sie kritisieren, dass bei der Einführung integrierter Modelle der Leistungserbringung meist nur über Änderungen in den Verfahren und Regelwerken sowie in den administrativen Systemen nachgedacht werde. Dies seien die Aspekte oberhalb der „Wasseroberfläche" eines Eisbergs, die für die Öffentlichkeit sichtbar sind (vgl. Corbett/Dimas/Fong/Noyes 2005: S. 33). Demgegenüber lege das Eisbergmodell nahe, dass auch Dimensionen „unterhalb der Wasseroberfläche“ auszugestalten seien. Diese Dimensionen sind Führungsstil, Organisationskultur und institutionelle Systeme. Dabei spielt die „Organisationskultur“ eine besondere Rolle: Wie nehmen die Menschen in den Programmen oder Agenturen sich selbst und andere wahr? Wie kommunizieren sie mit anderen innerhalb ihres Programms oder anderen, mit denen sie in beruflicher Beziehung stehen und welche Vokabeln werden dabei gebraucht? Welche Basisregeln bestimmen das institutionelle Leben?

26 Deswegen können die Ausführungen von Corbett/Noyes für die Diskussion um Netzwerke fruchtbar gemacht werden, auch wenn sie im Schwerpunkt die vollständige „Integration“ von Angeboten thematisieren. 
Corbett/Noyes belassen es aber nicht bei der Kritik, sondern verstehen ihre Arbeit als „formative Evaluation“, in der es nicht darum geht, den Erfolg oder Misserfolg eines definierten Programms oder Projekts festzustellen, sondern den Implementationsprozess zu begleiten und mit Hilfe der Ergebnisse der Evaluation auch zu gestalten. Hierzu ist eine Prozessperspektive geradezu zwingend. Vor dem Hintergrund der oben kurz skizzierten theoretischen Konzepte und unter Nutzung von Instrumenten aus der Betriebswirtschaft (strategisches Management, vgl. Bullinger/Warnecke 1996) entwickelte das Autorenteam ein System der Einleitung und Stabilisierung von Veränderungen mit dem Ziel, integrierte Dienstleistungen für Arbeitslose zu ermöglichen. Corbett/Noyes nennen ihren Ansatz „line of sight"-Perspektive. Ausgangspunkt ist die Vergewisserung der von allen Beteiligten erwünschten „Wirkungen“ der Angebote auf die jeweilige Zielgruppe. Analog zu Ansätzen des strategischen Managements wird nicht die aktuelle Situation (d. h. die existente „Angebotslandschaft") beleuchtet, vielmehr wird diese zu Gunsten visionärer Überlegungen eher ausgeblendet. Aus der Vision gewünschter Wirkungen werden die einzelnen Aktivitäten und die daraus resultierenden Anforderungen an alle Beteiligten abgeleitet. Die „line of sight“ wird aus der Perspektive der Leistungsadressatinnen und -adressaten definiert. Aus den jeweils zu erreichenden Grundsatzzielen werden ein (gemeinsam erarbeitetes) Zielsystem und dessen operative Umsetzung erarbeitet (vgl. ausführlich Abschnitt 4.7.1.2).

\subsection{Wie entstehen Produktionsnetzwerke?}

\subsubsection{Die Problemstellung}

Im vorigen Abschnitt wurde ein rekursives Verhältnis zwischen der Qualität von Netzwerken als Beziehungsstrukturen und dem Handeln der Akteure (Personen als Vertretungen von Organisationen und Organisationen als natürliche Personen) festgestellt. Damit gerät die Relation zwischen autonomer Organisation und Netzwerk in den Fokus der Betrachtung. Aus der Perspektive der Organisation kann das oben dargestellte, von Corbett und Noyes entwickelte Modell unterschiedlicher Stufen der Kooperation in doppelter Hinsicht genutzt werden:

- Pragmatisch für die Entscheidung, welche Intensität tatsächlich notwendig ist, um bestimmte vernetzte Leistungen zu realisieren. Dabei sollte sich diese Entscheidung am gewünschten Outcome orientieren 
und nicht an vorgegebenen Standards (vgl. Corbett/Noyes 2008: S. 12f.).

- Perspektivisch für die Abschätzung, welche Schwierigkeiten bei der Neukonstruktion von Dienstleistungsprozessen zu erwarten sind, wenn ein bestimmter Grad an Kooperation angesichts der spezifischen Organisationskultur der kooperierenden Organisationen erreicht werden soll.

Die bisherigen Überlegungen legen nahe, dass es nicht ausreicht, an der Spitze von Organisationen Kooperationsvereinbarungen abzuschließen, um Zusammenarbeit zu gewährleisten - Resultat wäre eine sehr instabile Konstruktion. Vielmehr müssen solche Vereinbarungen durch Verbindungen auf der Ebene der Organisationskulturen unterfüttert werden.

Wenn aber solche Verbindungen geschaffen werden, „verflüssigt“ sich die Organisationskultur, innerhalb von Organisationen gepflegte Routinen, Rituale und ggf. sogar „Core beliefs“ (kollektiv geteilte Wertvorstellungen) werden durch den intensiven Kontakt mit anderen Organisationen hinterfragbar. Impulse von außen regen die Entwicklung der Organisation an - die entscheidende Frage ist, ob diese Entwicklung gewollt oder spontan, gesteuert oder naturwüchsig verläuft. Eine solche Perspektive kann Abwehr auslösen - bei den Mitarbeitenden und bei der Organisationsspitze - und dazu führen, dass Kooperation trotz des erklärten Wunsches eher blockiert als gefördert wird. Auf alle Fälle ist mit einer solchen Rückkopplung von Kooperationsbeziehungen in die Organisation zu rechnen und es ist eine wichtige Aufgabe der Organisation, diese Rückkopplung zum Gegenstand reflexiven Handelns zu machen, indem z. B.

- Anlässe, Umfang und Intensität von Kooperationsbeziehungen geplant werden. Das bedeutet auch, durch die Definition von Zielgruppen und Einzugsbereichen (Sozialräumen) die Segmente der Organisation festzulegen, die erwartbar von den durch Kooperation induzierten Entwicklungen betroffen sein werden. Diese Segmente können dann als Experimentierfelder dienen.

- Die Kontakte zu Netzwerkpartnern zum Anlass gezielten organisationalen Lernens gemacht werden, das tatsächlich eigene Routinen, Rituale und Werte zur Disposition stellt. Organisationales Lernen kann nur funktionieren, wenn es von der Organisationsspitze gewollt ist und gefördert wird. Hilfreich sind hier Instrumente wie Hospitationen, gemeinsame Fortbildungen, gemeinsame Projekte o. ä. und die Reflexion der dabei gewonnenen Erfahrungen für die eigene Organisation.

- Kooperationsbeziehungen durch Personen gepflegt werden, die auf Gund ihrer hierarchischen Einbindung in der Lage sind, Impulse rasch 
und mit Nachdruck in die Organisation weiterzuleiten. Es können Stabsstellen geschaffen oder Projektgruppen installiert werden - in allen Fällen geht es darum, Kooperation systematisch aufzubauen und zu nutzen und die irritierenden Effekte produktiv zu verarbeiten. Das bedeutet auch, dass die Personen, die mit diesen Aufgaben betraut sind, über Kompetenz im doppelten Wortsinne verfügen müssen: Fachkenntnisse im Hinblick auf Projekt- und Netzwerkmanagement und innerorganisatorische Entscheidungskompetenz.

Wenn Kooperation organisationskulturell verankert werden soll - und dies ist eine wesentliche Erfolgsbedingung -, dann muss sie von der Organisationsspitze unterstützt und gefördert werden. Dies setzt nicht nur den viel zitierten Willen, sondern auch die Bereitstellung von Ressourcen voraus, die geeignet sind, die innerorganisatorischen Bedingungen interorganisationaler Kooperation zu sichern.

\subsubsection{Die Kooperation sozialer Dienstleistungsorganisationen als Anforderung im Arbeitsalltag}

\subsubsection{1 „Verteilte Expertise“}

Die Herausforderung der Kooperation zwischen Organisationen im Bereich sozialer Dienstleistungen stellt sich auf zwei Ebenen:

- Als Kooperation zwischen zwei (oder mehreren) Organisationen mit jeweils spezifischen Zwecken, Hierarchien und Mitgliedschaftsbedingungen, die sich in unterschiedlichen Aufbau- und Ablauforganisationen niederschlagen.

- Als Kooperation zwischen Beschäftigten dieser Organisationen, die vor dem Hintergrund der jeweiligen spezifischen Arbeitsbedingungen handeln und möglicherweise über einen unterschiedlichen beruflichen Hintergrund und ein spezielles Professionswissen (vgl. Fried 2003) verfügen.

Die Herausforderung ist dann besonders hoch, wenn es darum gehen soll, dauerhafte und verbindliche Netzwerkstrukturen zu schaffen und zu stabilisieren. Und die Herausforderung wird noch gesteigert, wenn die zu vernetzenden Organisationen unterschiedlichen organisationalen Feldern ${ }^{27}$

27 Mit dem Begriff „organisationales Feld“ bezeichnen die amerikanischen Soziologen DiMaggio und Powell ,jene Organisationen, die gemeinsam einen abgegrenzten Bereich des institutionellen Lebens konstituieren: die wichtigsten Zulie- 
angehören, unterschiedliche professionelle Arbeitsweisen zur Anwendung kommen, unterschiedliche professionelle Standards gelten und unterschiedliche Finanzierungsgrundlagen oder auch unterschiedliche gesetzliche Aufträge vorliegen.

Um diese Problematik theoretisch zu vertiefen, können Erkenntnisse zum Zusammenhang von Professionswissen, Organisation und interinstitutioneller Kooperation genutzt werden, die im Rahmen eines in England durchgeführten Forschungs- und Entwicklungsprojekts gewonnen werden konnten. Hintergrund war das im Jahre 2004 vom dortigen Familienministerium aufgelegte Programm zur Verhinderung der sozialen Ausgrenzung von Kindern. Ein wesentlicher Bestandteil des Programms war die Förderung interinstitutioneller Kooperation zwischen Akteuren der Jugendhilfe, Schulen, Kinderpsychologen und anderen Institutionen, die mit Kindern und Jugendlichen befasst sind. Im Rahmen des Programms wurden etliche wissenschaftliche Untersuchungen durchgeführt und Literatursurveys erstellt, die sich mit Formen und Problemstellungen interinstitutioneller Kooperation beschäftigten (vgl. Warmington u.a. 2004). Eine dieser Untersuchungen wurde im Rahmen des Projekts „Learning in and for Interagency Working“ von 2004 bis 2007 von einem Team der Universitäten von Bath und Birmingham durchgeführt (vgl. Edwards u. a. 2009). Die Studie verfolgte zwei Fragestellungen: Was und wie lernen Fachkräfte im Zusammenhang mit der Entwicklung kooperativer Antworten auf das Problem drohender Ausgrenzung von Kindern? Welche organisatorischen Bedingungen sind hierbei förderlich? Das Forschungsteam greift auf die Tätigkeitstheorie Engeströms zurück, um ihr eigenes Forschungsprogramm zu modellieren (vgl. Engeström 2008a; 2008b; 2008c; 2008d). Zentraler Ausgangspunkt ist die Konzeption der „,verteilten Expertise“ („distributed expertise“), d. h. eines professionellen Wissens über Kinder und ihre Lebensumstände, das von einzelnen Professionen (Sozialarbeiterinnen und Sozialarbeitern, Psychologeninnen und Psychologen, Lehrende) getrennt voneinander generiert wurde. Erst wenn es gelingt, dieses isolierte Wissen zusammenzubringen, kann - so die zentrale These - interinstitutionelle Kooperation gelingen. Dann findet etwas statt, was Edwards u. a. „Ko-Konfiguration" nennen (Edwards u.a. 2009), die Entstehung eines gemeinsam geteilten, komplexen Blicks auf den „Gegenstand“.

ferfirmen, Konsumenten von Ressourcen und Produkten, Regulierungsbehörden sowie die Organisationen, die ähnliche Produkte oder Dienstleistungen herstellen bzw. anbieten“ (DiMaggio/Powell 2009: S. 149). 
Wissenssoziologisch formuliert geht es um das Überschreiten der geistigen Schemata der einzelnen Professionen. Dabei kommt es nicht darauf an, einen „kleinsten gemeinsamen Nenner“ zu finden, sondern die „verteilte Expertise" als solche zu akzeptieren und zu verknüpfen. Ein Problem für interinstitutionelle Kooperation stellt nach Auffassung des Autorenteams die „figured world“ der Organisationen dar. Im Rückgriff auf sprachtheoretische Konzepte Basil Bernsteins beschreiben sie deren „tacit psychological work through their discursive practices, that is, how they talk, interact and use resources" (Edwards u.a. 2009: S. 57). Bernstein unterscheidet die strukturelle und die interaktionale Ebene von Organisationen. Die strukturelle Ebene wird geformt durch die Definition von $\mathrm{Zu}-$ ständigkeiten und Organisationsgrenzen sowie durch entsprechende lokale Sprachregelungen. Die interaktionale Ebene betrifft die Kommunikation in der Institution, die sozialen Beziehungen am Arbeitsplatz. Hier geht es um innerorganisatorische Kontrolle durch die Definition von hierarchischen Positionen und Setzung von Rahmenbedingungen für das Handeln der Organisationsmitglieder. Regulative Diskurse sorgen dabei für die Vermittlung von Normen: „Regulative discourse is of particular relevance to the development of inter-professional practices as it communicates an institution's public moral practice, value beliefs and attitudes, principles of conduct, character and manner" (Edwards u.a. 2009: S. 60). Die theoretischen Querverbindungen zu den Analysen von Corbett und Noyes sind nicht zu übersehen.

Interorganisatorische Kooperation kann durch organisatorische Bedingungen eingeschränkt werden, fördert umgekehrt aber unter Umständen auch Organisationslernen, insbesondere dann, wenn Spannungen zwischen dem (durch Kooperation erweiterten) Professionswissen und den organisatorischen Sprachregeln auftreten. Im Kontext des Projekts kam es zu einem „Focussing on the whole child in the wider context“", unterschiedliche professionelle/institutionelle Sichtweisen wurden benannt und verhandelt - auf diese Weise gelang es, den Gegenstandsbereich ("object of activity") zu erweitern (vgl. Edwards u.a. 2009: S. 67). Kooperationsstrukturen können dann durch die Entwicklung gemeinsamer Instrumente, z. B. Dokumentationssysteme, stabilisiert werden. Dabei handelte es sich nicht um technische Vorgänge, vielmehr ist diese Entwicklungsarbeit eng verknüpft mit der Ausdehnung von professionellen Wissensbereichen. Im Zuge dieser Arbeit kann es zu Rückwirkungen in die beteiligten Organisationen kommen: die „figured worlds“ verändern sich. 


\subsubsection{2 „Fälle“ als Zugänge zu geteilten Realitäten}

Nach der Theorie Engeströms, auf die sich Edwards u. a. explizit beziehen, konstruieren Organisationen die „Gegenstände“ ihrer Tätigkeit in einem komplexen Zusammenhang aus formaler Struktur, Arbeitsaufträgen, Organisationskultur, professionellen Wissensbeständen etc. (vgl. Engeström 2008a). Soweit es zur Ausbildung von Kooperationsstrukturen kommt, bezieht sich diese auf gemeinsame "Gegenstände“, die allerdings aus einem jeweils spezifischen Organisationskontext heraus konstruiert werden. „KoKonfiguration“ bedeutet deshalb die (unter Umständen langwierige) Herausbildung eines gemeinsam geteilten Blicks auf diesen „Gegenstand“.

Im Kontext von Sozial-, Arbeitsmarkt- und auch Migrationspolitik sind unterschiedliche „Gegenstände“ denkbar, die zur Kristallisation „verteilter Expertise“ beitragen könnten. Prominent sind dabei natürlich genau die Themen, die zur Etablierung organisationaler Felder beigetragen haben, d. h. von mehreren Organisationen arbeitsteilig und zum Teil kooperativ, zum Teil konkurrierend bearbeitet werden: Z. B. aufenthaltsrechtliche Fragen, Arbeitslosigkeit, Armut, Kinderbetreuung, Sprache und Bildung, Wohnungsversorgung. Sie bilden u. a. den Ausgangspunkt für Diskurse, die im Rahmen von Sozialplanung, Jugendhilfeplanung und anderen Fachplanungen geführt werden. Diese Diskurse stützen sich auf Daten, die überwiegend aus Expertensystemen gewonnen werden, wie z. B. der Sozialberichterstattung. Merkmale dieser Daten ist es, dass sie sich auf einen abstrakten Merkmalsraum beziehen, „Strukturen“ abbilden und gerade deshalb von individuellen Lebensverhältnissen abstrahieren. Strukturen sozialer Ungleichheit und lokaler Versorgung wirken sich zwar im Einzelfall aus, werden aber fallunabhängig generiert. Sie werden über die Analyse sozialstatistischer und ökonomischer Daten fassbar, die systematisch von Besonderheiten absehen. Die Programmatik sozialstaatlicher Intervention setzte (im 19. Jahrhundert in bewusster Abgrenzung zum individualisierenden Fürsorgegedanken) an diesen überindividuellen Strukturen an, beabsichtigte die Beeinflussung rechtlicher und/oder ökonomischer Strukturen und die Gestaltung von „Sozialräumen“. Seit den 80er Jahren des 20. Jahrhunderts hat sich kommunale Sozialplanung entwickelt, deren Idee es war, auf sozialräumlicher Ebene Infrastruktur zu gestalten, während der Bezug auf Einzelfälle eher akzidentiell blieb. Diese Perspektive der Sozialplanung ist weiterhin notwendig, aber nicht hinreichend, solange es nicht gelingt, eine konzeptionell triftige Verbindung zwischen Struktur und „Fall“ herzustellen. Denn obwohl die Praxis der Arbeitsförderung, der Kinder- Jugend- und Altenarbeit und anderer Felder von Sozial- und Arbeits- 
marktpolitik seit langer Zeit flankiert ist von infrastrukturell ausgerichteter Bearbeitung von Problemlagen, besteht eine konzeptionelle, häufig auch institutionelle und organisatorische Trennung zwischen Fallarbeit und Fachplanung, individueller Hilfe und sozialpolitischer Intervention.

Die pädagogische Intervention (vgl. Kaufmann 2012) ergänzte immer schon die an Strukturen ansetzende sozialpolitische und sozialplanerische Programmatik, wurde aber nie zur zentralen Figur sozialpolitischer Gestaltung. Dies änderte sich partiell mit der Ausdifferenzierung von Lebenslagen und international wirksamen Programmatik der „Aktivierung“. ${ }^{28}$ Der Grundgedanke pädagogischer Intervention ist es, Personen dabei zu unterstützen, ihr Leben selbstständig führen zu können und gleichzeitig die Grundlagen dafür zu erhalten, sich in ihrer Persönlichkeit (z. B. durch Bildungsprozesse) entwickeln zu können. Dies setzt zwingend Ko-Produktion voraus, d. h. die Interaktion zwischen den Fachkräften und den adressierten Personen dieser Hilfe. Grundlage dieser Ko-Produktion ist von Seiten der Fachkräfte das Verstehen der Adressaten, was in der Tradition der Sozialen Arbeit als Empathie gefasst wird.

Es gilt, die Trennung von Fallperspektive und Strukturperspektive aufzuheben, damit der gemeinsame "Gegenstand“ der Arbeit unterschiedlicher Professionen und Organisationen in den Fokus genommen werden kann: die (hilfebedürftige) Person in ihren sozialen (und damit strukturell geprägten) Lebensbedingungen. Im Einklang mit den Ansätzen von Corbett und Noyes, Edwards u.a. sowie Engeström plädiert der Autor dieses Leitfadens dafür, die Aufhebung der Trennung dadurch in Angriff zu nehmen, dass planerische Aktivitäten an alltäglichen Fällen ansetzen. Hierfür gibt es im Wesentlichen drei Gründe:

- Einzelfälle und ihre Geschichte bieten konkrete Anhaltspunkte dafür, unterschiedliche Perspektiven zu entwickeln und am konkreten Material zu explizieren. Sie bieten einen „Überschuss an Anknüpfungen. Dies erlaubt anders als bei hoch selektiven, standardisierten Datensätzen, Varianz in der Nutzung und Verwertung. So lässt eine Geschichte verschieden gerichtete Demonstrationen, Schlüsse und Urteile zu. (...) Der Überschuss an Zeichen, Markern, Modulen etc. offeriert vielfältige (Re-)Konstruktionen einerseits“" (Scheffer 2014: S. 245).

- Die Einzelfälle sind Konstruktionen unterschiedlicher, am „Fall“ beteiligter Professionen; keine Profession kann substanziell eine Deutungshoheit in Anspruch nehmen (auch wenn dies von verschiedenen Pro-

28 Nicht zufällig lautet der Titel eines Buches von van Berkel und Valkenburg (2007) über die Arbeitsmarktpolitik in der EU „Making it personal“. 
fessionen immer wieder in Anspruch genommen wird - aber dieser Anspruch bleibt nie unangefochten). Der Diskurs über einen „Fall“ ist diesem bereits inhärent - was genutzt werden kann, um die „verteilte Expertise“ als komplexe Expertise zu realisieren.

- Die konkrete Tätigkeit bildet den Berührungspunkt unterschiedlicher Organisationen, die für dieselben Personen Leistungen erbringen. Bei näherer Betrachtung zeigt sich rasch, dass die Fachkräfte unterschiedlicher Organisationen mit der gleichen Person (oder Familie) arbeiten, dies aber auf durchaus unterschiedliche Weise tun. Der scheinbar „gemeinsame Fall“ erweist sich so als eine Ansammlung organisationsspezifischer „Fälle“, deren Gemeinsamkeit erst noch herausgearbeitet werden muss.

Die gemeinsame Bearbeitung von „Fällen“ ist hilfreich aber nicht zureichend, um eine gemeinsame Perspektive zu entwickeln. Das Besondere muss auf das „Allgemeine“ bezogen werden und das Allgemeine besitzt eine den Einzelfall übersteigende Bedeutung. Dieser komplexe Zusammenhang soll im Folgenden näher erläutert werden.

\subsubsection{Was ist der „Fall“?}

Ein „Fall“ entsteht allgemein, „wenn Einzelnen, Gruppen oder Gemeinschaften aus ihrer eigenen Perspektive heraus oder aber aus der Perspektive der Gesellschaft heraus ein Problem angeheftet wird, das es zu lösen gilt“ (Giebeler 2007: S. 11). Ein „Fall“ ist somit stets eine soziale Konstruktion: „Eine Person ist kein Fall und entsprechend spricht, arbeitet oder schläft ein Fall nicht. Vielmehr (...) können diese Lebensäußerungen einer Person (...) nur vor einem bestimmten thematischen und/oder interventionspraktischen Hintergrund für einen beobachtenden Dritten zum Fall werden“ (Maiwald 2008: S. 5).

Die Theorie der Konstruktion sozialer Probleme bietet einen Bezugsrahmen, um diesen Zusammenhang weiter zu vertiefen. Dem Sozialkonstruktionismus ${ }^{29}$ geht es generell darum, die Entstehung sozialer Probleme als

29 Groenemeyer verwendet den Begriff „Sozialkonstruktionismus“. Derselbe Ansatz wird jedoch auch als "Sozialkonstruktivismus“ bezeichnet. Die Theorie der Konstruktion sozialer Probleme bietet einen Bezugsrahmen, um diesen Zusammenhang weiter zu vertiefen. Dem Sozialkonstruktivismus geht es generell darum, die Entstehung sozialer Probleme als Resultat gesellschaftlicher Konstruktionen zu analysieren. Im Fokus steht die Erkenntnis, dass gesellschaftliche Ordnung und kulturelles Wissen nicht a priori existieren, sondern durch menschliche Kon- 
Resultat gesellschaftlicher Konstruktionen zu analysieren. Einige Autorinnen und Autoren heben insbesondere die Bedeutung interaktiver institutioneller Prozesse hervor und bezeichnen diese als Problemarbeit. In der Sozial-, Gesundheits- und Bildungsarbeit spielen neben dem Alltagswissen und dem Professionswissen der Akteure institutionelle und organisationale Regeln und Wissensbestände eine wichtige Rolle. „Doing social Problems ist die Anwendung von Regeln, Techniken und Wissen auf individuelle Problemlagen und Problemsituationen. Grundlage hierfür ist ein Prozess der Kategorisierung und ihrer Begründung in Rahmen von legitimierten Wissensbeständen, die für die Institutionen der Problembearbeitung typisch sind. Hierzu wird in der Regel auf Gesetzestexte und Vorschriften, Diagnosehandbücher, Risikochecklisten oder Programme zurückgegriffen, die als ein selbstverständliches Wissen routiniert angewendet werden und die Grundlage für Aushandlungsprozesse mit den Betroffenen darstellen“ (Groenemeyer 2010: S. 17, Hervorhebung im Original). Damit geraten auch strukturelle Machtverhältnisse in den Blick. Der Prozess der Konstitution sozialer Probleme wird als mehrstufiger Prozess gesehen; die Analyse erstreckt sich über unterschiedliche Ebenen, Felder oder Arenen, „die jeweils spezifische Kontexte und Rahmenbedingungen für Problematisierungen und damit auch für die Arbeit in Institutionen und Organisationen der Problembearbeitung darstellen. Für die Analyse der Mobilisierung von Öffentlichkeit sind offenbar andere Konzepte notwendig als für die Analyse der Konstruktion und Bearbeitung politischer Issues innerhalb der Organisationen des politischen Systems. Schließlich folgt die alltägliche Problemarbeit des Doing social Problems anderen Bedingungen und folgt einer anderen Logik als die politische Institutionalisierung von Problemkategorien“ (ebd.). Die Ebenen folgen verschiedenen Logiken, sind aber systematisch miteinander verbunden. „Für Institutionen der Problembearbeitung sind öffentliche Diskurse und die in ihnen etablierten Problemkategorien von Bedeutung, insofern sie die Organisationen mit Legitimation und Reputation für die von ihnen durchgeführten Maßnahmen und Angebote versorgt" (ebd.: S. 29).

Im Prozess der politischen Bearbeitung formulierter „legitimer“ Probleme bilden sich politische Domänen, die unter Umständen die Ausbildung organisationaler Felder begünstigen. „Dabei handelt es sich nicht nur um die organisatorische Arbeitsteilung von Zuständigkeiten in den Organisationen des politischen Systems (Ressorts, Abteilungen u. ä.), sondern um

struktionsleistungen produziert werden. Gesellschaftliche Ordnung unterliegt also einer Logik der Kontingenz. 
mehr oder weniger institutionalisierte Netzwerke von an einem Problem interessierten und als kompetent angesehenen Akteuren innerhalb und außerhalb des politischen Systems. Zu diesen Netzwerken gehören insbesondere entsprechende Abteilungen oder Ressorts innerhalb des politischen Systems, aber auch zum Beispiel Wohlfahrtsverbände, Interessenverbände, wissenschaftliche Experten und Expertinnen sowie besonders im Feld engagierte Vertreter und Vertreterinnen von Medien und sozialen Bewegungen, sofern diese einen gewissen Organisationsgrad und eine organisatorische Verlässlichkeit erreicht haben. Besonders auf lokaler Ebene sind auch Vertreter und Vertreterinnen der Institutionen der Problembearbeitung dauerhaft in die spezifischen Politiknetzwerke integriert" (ebd.: S. 31f.).

Die gesellschaftlichen Diskurse werden auf diese Weise durch die Institutionen und Organisationen gefiltert. Nach Auffassung der hier zitierten Vertretungen des Konstruktionismus sind es die institutionellen Settings, die letztlich den entscheidenden Einfluss auf das Handeln der Akteure haben:,Not only are social problems representations organizationally produced and preferred models for interpretations, but their use is conditioned by prevailing local preferences, practices and ressources. Both image and attachment are organizationally embedded (...); categories and practices through which they are applied reflect local interpretative circumstances and culture" (Holstein/Miller 2003: S. 87). Die Analyse des Handelns der Fachkräfte wird auf diese Weise systematisch mit der Analyse diskursiver Praktiken verknüpfbar: sowohl die konkrete Problemarbeit im Organisationsalltag wie auch Organisationsstrukturen und Masterdiskurse werden Gegenstand der Analyse. „Die Interpretationen der Problemarbeiter sind geformt durch die interpretativen Strukturen und Ressourcen, die lokal verfügbar und akzeptabel sind. In der konkreten, problemkonstituierenden Fallbeschreibung - wie zum Beispiel derjenigen eines jugendlichen Delinquenten - werden jeweils verfügbare Ressourcen nutzbar gemacht, die im Prozess der Sinnzuschreibung zur Anwendung kommen (...). Damit verläuft Problemarbeit quasi in organisatorisch vorgezeichneten Bahnen, während die Akteure sich bemühen, alltagspraktischen Belangen gerecht zu werden" (Schmidt/Hasse 2010: S. 60f.). Organisationen sehen konkrete Personen als „Fälle“, d. h. nehmen die Lebenswirklichkeit und die Subjektivität von Menschen nach Kriterien (selektierend) wahr, die an eigenen oder übernommenen (z. B. gesetzlich definierte Zuständigkeiten) Regeln ausgerichtet sind. Hinzu kommen von Seiten der Mitarbeiterinnen und Mitarbeiter unter Umständen professionell geprägte Wahrnehmungsmuster. Die Bearbeitung von Fällen erfolgt nach organisatorisch und/oder 
professionell geprägten Handlungsmustern - hier manifestiert sich die „verteilte Expertise“ (vgl. Abschnitt 4.4.2.1).

Schaubild 6: Wissensproduktion - Konstitution und Reflexion von „Fällen"

Organisation

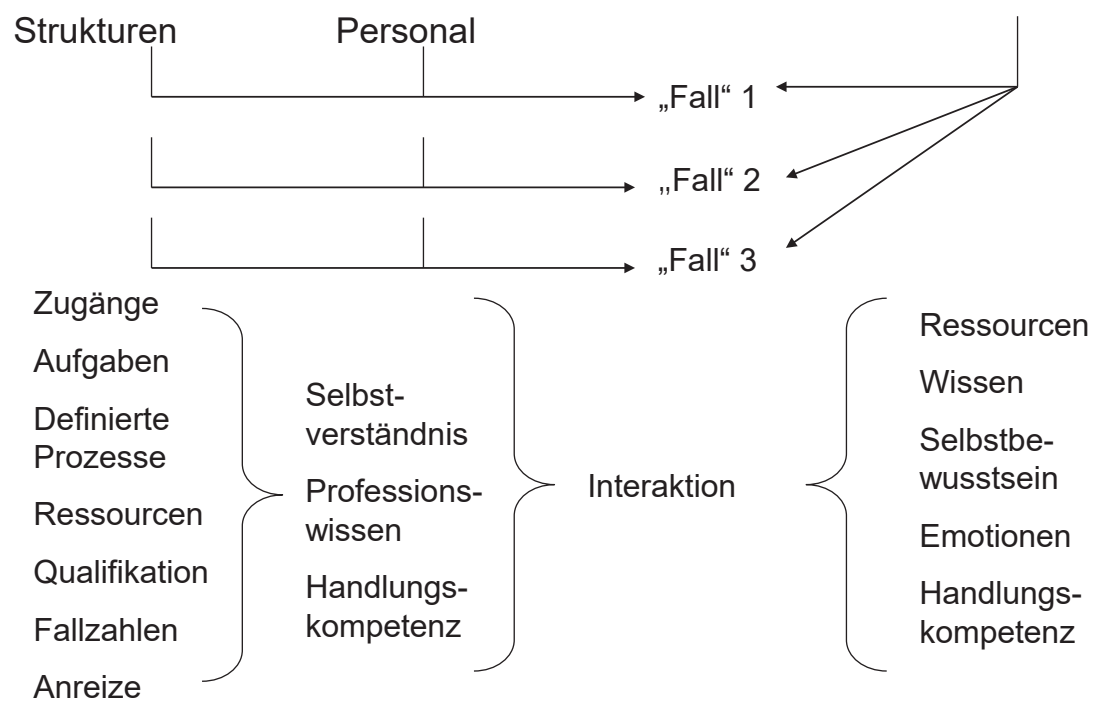

(Eigene Darstellung)

Damit vollzieht sich die situative Interaktion zwischen Personen, der Fachkraft einerseits und dem/der Leistungsadressaten/in andererseits, innerhalb strukturell geformter Bedingungen - sie ist zwar spezifisch, aber nicht von den strukturellen Bedingungen ablösbar, die auch auf Seiten der Adressatinnen und Adressaten (also der Person) wirken. Treffen Personen auf mehrere Organisationen bzw. deren Vertreterinnen und Vertreter, werden mehrere „Fälle“ parallel (aber nicht unbedingt gleichzeitig) konstruiert und konstituiert. In jedem „Fall“ wird je ein spezifisches Bild der Person gezeichnet, das handlungsleitend für die jeweilige Interaktion ist und damit rekursiv die Wahrnehmungs- und Handlungsstrukturen der Beteiligten stabilisiert (aber auch unter Umständen modifiziert) (vgl. Schaubild 6).

Es reicht jedoch nicht aus, aus mehreren „Fällen“ einen „Fall“ zu machen, d. h. eine mehrperspektivische Betrachtung anzustreben. In diesem Falle würde die Basis des organisatorisch geprägten selektiven Blicks erweitert - aber die Person, die zum „Fall“ geworden ist, dennoch verfehlt, da die Nutzerperspektive fehlt, d. h. die Perspektive der Person, die zur Leis- 
tungsadressatin/zum Leistungsadressaten wurde. Um zu einer (annähernd vollständigen) integrierten Darstellung einer Fallkonstellation (bestehend aus mehreren, aus der Perspektive der Organisationen definierten „Fällen“) zu kommen, muss deshalb die Nutzerperspektive eingeführt werden - entweder direkt (durch die unmittelbar Betroffenen) oder indirekt durch die Rekonstruktion von Sinnzusammenhängen, die sich an der Fallgeschichte zeigen, die aber nicht rein subjektiv, d. h. einer Rekonstruktion nicht zugänglich sind.

\subsubsection{Der „Fall“ als das „Allgemeine im Besonderen“}

In einem „Fall“ kreuzen sich immer subjektive und objektive Perspektiven, geht es einerseits um Personen mit ihrem subjektiven Handeln, ihren Motiven, ihrem subjektiv gemeinten Sinn und andererseits um den objektiven Sinn, den diese Handlung für andere hat. Ein „Fall“ bezieht sich in der Sozialen Arbeit (wie in der Pädagogik) somit immer auf Subjektives, das Besondere, stellt dieses aber in einen breiteren Kontext - nur dadurch kann von „Fall“ die Rede sein: „Jeder Fall ist das Resultat hoch selektiver Prozesse der Zurichtung und Abstraktion. Ereignisse sind in ihrer Urform und in der Gestalt, in der sie (...) zum Fall werden, durchsetzt und geprägt von persönlichen Eigenarten, besonderen Umständen, situativen Zufällen etc., die es schwer, wenn nicht unmöglich machen, das Geschehen umgehend als ,Fall von X' zu identifizieren. Deshalb besteht ein wichtiger Schritt der Fallkonstruktion darin, diese kontingenten Elemente auszuwaschen" (Bergmann 2014: S. 428). Aber: „der Fall existiert zwar nur qua Subsumption, er geht jedoch in der Subsumption nicht auf. Auch wenn er seine Bestimmung als ,Fall von X', also durch Bezug auf einen allgemeinen Satz erhält, ist ihm ein Überschuss an Sinn eigen, der ihm einen eigenen epistemischen Status verleiht" (ebd.: S. 429). Giebeler versucht dieses komplexe Verhältnis durch eine begriffliche Differenzierung zu fassen. Sie unterscheidet „Fallarbeit“, „Fallreflexion“ und „Fallrekonstruktion“, wobei die beiden erstgenannten Begriffe auf das Bemühen zielen, die Besonderheit in den Fokus zu stellen, eine Beziehung zwischen Personen zu stiften (vgl. Giebeler 2007: S. 13). Fallrekonstruktion hingegen setzt am „Fall“ an, beansprucht aber "gesicherte Aussagen treffen zu können - und zwar zum einen zu dem jeweils konkreten ,Fall', aber auch über den Einzelfall hinaus, indem Typologien zu weiteren und zukünftigen Fällen eines bestimmten Feldes entwickelt werden. Der Rekonstruktion geht jedoch die 
Konstruktion voraus - die Konstruktion sozialer Begebenheiten als ,Soziales Problem““ (ebd.: S. 15).

Damit sind aber zwei Ebenen bezeichnet:

- Die Ebene der Interaktion, in der die Subjektivität der Klientinnen und Klienten zum Zuge kommt, der besondere, subjektive Sinn ihrer Handlungen im Fokus steht und in Verbindung gebracht wird zu den (teils damit in Widerspruch stehenden) objektiven Bedeutungen dieser Handlungen. Es gehört zum „Fallverstehen“, diesen subjektiven Sinn so gut es geht zu erschließen.

- Die Ebene der Strukturrekonstruktion, in der nicht nur die sozialen und rechtlichen Bedingungen identifiziert werden, die erst den „Fall“ ausmachen, sondern auch subjektive Verhaltensweisen als Manifestationen von Handlungstypen angesehen werden können.

Diese beiden Unterscheidungen sind mit zwei Varianten der Hermeneutik in Verbindung zu bringen:

- Eine an der Traditionslinie von Schleiermacher und Dilthey orientierte „innere Teilhabe“ an einer konkreten lebensweltlichen Beschreibung, dem Nachbilden „fremden Seelenlebens“ (Dilthey 1977: S. 265, zitiert nach Wernet 2006: S. 56): „das Ideal ist nicht die Nachkonstruktion, sondern das Nacherleben; das Hineintauchen in die Lebenswelt; das Ideal ist die weitestgehende Assimilation des Forschers an die zu erforschende Welt" (Wernet 2006: S. 56, Hervorhebung im Original). Hier sind klare Bezüge zu dem zu sehen, was in Psychologie und Sozialer Arbeit als Empathie bezeichnet wird.

- Eine Variante, in der „eine konkrete Erscheinung (...) weder als bedeutungslose, kontingente Singularität aufgefasst werden kann, noch als bloßes Exemplar einer allgemeinen Regularität" (ebd.: S. 57). Es geht somit nicht um das unverwechselbare „Einzelne“, sondern um den Einzelfall als spezifisches Exemplar eines strukturellen Zusammenhangs, der als solcher rekonstruiert werden kann. Gleichwohl ist dieser „Fall“ nicht mit der Person identisch, die seinen Kern ausmacht. „Der Fall (...) ist eingespannt in die Dialektik von Allgemeinem und Besonderem“ (ebd.: S. 58).

Letzteres zielt auf die überindividuellen, strukturellen Momente des „Falles“: „Um den Sinngehalt geistiger Gebilde zu erschließen, ist es (...) weder hinreichend noch unbedingt notwendig, die individuellen Motive, Vorstellungen und Überzeugungen ihrer Erzeuger in Erfahrung zu bringen: nicht hinreichend, weil darin die objektiven Bedeutungsüberschüsse nicht enthalten sind; nicht unbedingt notwendig, weil die Kenntnis der Absichten und Antizipationen des Urhebers zwar den Zugang zum Sinngehalt 
seines Werkes erleichtern, in vielen Fällen jedoch auch den Blick für dessen objektive Bedeutung verstellen kann“ (Schneider 2009: S. 30f., Hervorhebung im Original). Für die auf der Basis von Empathie handelnden Fachkräfte ist es allerdings sehr wohl notwendig, „die individuellen Motive, Vorstellungen und Überzeugungen in Erfahrung zu bringen“ - sie wollen (und sollen) den subjektiv gemeinten Sinn des Handelns ihrer Klientinnen und Klienten erschließen, um effektiv individuelle Unterstützung leisten zu können. Hier liegt die Differenz von „Fallverstehen“ als Verstehen des subjektiven Sinns, mit dem soziale Akteure ihr Handeln unterlegen, und der „Fallrekonstruktion“, die auf Strukturelles zielt und versucht, den Einzelfall auf Allgemeines zu beziehen.

Die beiden Seiten des „Falles“, das Besondere wie das Allgemeine, sind ko-präsent, können aufeinander bezogen, aber auch voneinander getrennt werden: „Man kann einen Fall in seiner Beispielhaftigkeit wahrnehmen, indem man ihn vergleichend neben andere Fälle hält, mit dem Ergebnis, dass er als, ein Fall von $X^{\prime}$ identifiziert wird und seine Identität durch den Bezug auf eine allgemeine Regel erhält. Man kann einen Fall aber auch in seiner Einzigartigkeit wahrnehmen, indem man ihn - abgekoppelt von Bezügen auf allgemeine regeln - als biografische Einheit über die Zeit konstruiert, wodurch er in seiner Individualität und Besonderheit (...) hervortritt" (Bergmann 2014: S. 429). Fallverstehen und Fallrekonstruktion können somit methodisch voneinander getrennt werden - um die strukturellen Aspekte eines Falles zu bearbeiten, muss nicht der subjektiv gemeinte Sinn bis in alle Verästelungen erschlossen werden, letzteres kann aber sehr wohl Thema einer Fallkonferenz sein, die auf individuell zugeschnittene Unterstützung abzielt, während sich die Planungskonferenz dem „Fall“ in seiner allgemeinen Bedeutung widmet und aus mehreren „Fällen“ Konsequenzen für das System/die Sozialplanung ableitet. Die Fallrekonstruktion liefert also das Material für Planungen, ergänzt, bereichert und konterkariert somit systematisch die Daten, die im Rahmen von Sozialberichterstattung gewonnen und aufbereitet werden. Die pädagogische Kasuistik ist ,in der Lage, in aktuellen und konkreten Entscheidungssituationen Anhaltspunkte für die Zukunft zu geben und Wegweiser zu sein“" (Hörster 2010: S. 378).

\subsubsection{Die Planung von Leistungsprozessen}

Die Fallrekonstruktion bildet den Ausgangspunkt, um in institutionsübergreifende Planungsprozesse einzutreten, die über die Bearbeitung von Ein- 
zelfällen hinausgehen. Resultat dieser gemeinsamen Planung (z. B. in Planungskonferenzen) kann die Entwicklung gemeinsamer Leistungsprozesse sein, in denen fallübergreifend Schnittstellen zwischen Einzelleistungen verschiedener Organisationen definiert und Übergänge geregelt werden (vgl. Reis u. a. 2011):

- Es gilt, an konkreten Fallkonstellationen die Selektivität der eigenen professionellen bzw. institutionellen Perspektive zu erkennen und zu einer Aufhebung der „verteilten Expertise“ zu gelangen, um eine komplexere Fallsicht zu realisieren. Hierzu gehört auch die Einbeziehung der Perspektive der Nutzenden. Diese Erweiterung der Perspektive ermöglicht eine Überprüfung organisationaler Routinen und Prozesse „double-loop-learning“ im Sinne von Argyris (vgl. Argyris 2002).

- Diese Überprüfung ist eingebettet in den Aufbau einer Netzwerkstruktur, die verbindliche organisationsübergreifende Kooperationen fördert - und gemeinsame planerische Aktivitäten beinhaltet.

- Beides ist nur möglich, wenn tradierte professionelle und administrative Denkgewohnheiten überwunden und Fall- und Planungsperspektive verbunden werden.

Ein wichtiges Element des Konzeptes Produktionsnetzwerk sind deshalb Planungskonferenzen. Während sich Fallkonferenzen mit Einzelfällen auseinandersetzen, ist es für Planungskonferenzen wichtig, aus einer (überschaubaren) Anzahl von Einzelfällen gesicherte Aussagen über Strukturen generieren zu können, die dann zum Gegenstand gemeinsamer Planungen werden können. Hierzu kann auf eine Idee zurückgegriffen werden, die der brasilianische Pädagoge Paolo Freire entwickelt hat. Die Idee besteht darin, im Zuge pädagogischer Arbeit (z. B. im Kontext von Alphabetisierungskursen) die „generativen Themen“ zu identifizieren, die das Leben und die Lebensverhältnisse der Menschen bestimmen. Diese sollten nicht von den Pädagogen vorgegeben, sondern in der gemeinsamen Arbeit entwickelt werden (vgl. Freire 1991). In ähnlicher Weise werden zunächst Einzelfälle, die von den Fachkräften als typisch angesehen werden, gemeinsam rekonstruiert. Auf der Basis einer Anzahl von Fällen werden dann fallübergreifende Themen benannt und "generative Themen" herausgearbeitet, die gemeinsam im Akteursnetzwerk bearbeitet und zum Gegenstand von Planungen gemacht werden. Dabei ist der systematische Unterschied zwischen den fallübergreifenden Themen und den „generativen Themen" eines Netzwerks zu beachten: Fallübergreifende Themen ergeben sich aus der Rekonstruktion eines Sets von Fällen, die als „typische Fälle" deklariert wurden. Generative Themen umfassen neben den fallübergreifenden Themen auch Themen, die in den bearbeiteten Einzelfäl- 
len gar nicht oder nur am Rand vorkommen, sich aber z. B. über Ergebnisse von Jugendhilfeplanung oder Sozialplanung zeigen, z. B. ein hohes Mietniveau, die soziale oder demografische Struktur eines Stadtteils. Denn die Arbeit an Einzelfällen darf nicht zu dem systematischen Fehlschluss führen, „Fälle“ zum Ausgangspunkt von Planungen zu machen, gleichzeitig aber die strukturellen Lebensbedingungen dann auszublenden, wenn sie in den typischen „Fällen“ nicht thematisiert werden. Zwischen den Themen aus typischen „Fällen“ und den thematisierbaren strukturellen Bedingungen kann, aber muss nicht, eine Schnittmenge bestehen. Deshalb muss die klassische, an Strukturen ansetzende Planungsperspektive auch in die Planungskonferenzen hereingenommen werden. Resultat dieser Arbeit ist ein Themengerüst, das den Ausgangspunkt für gemeinsame Planungen darstellt. Allerdings ist zu beachten, dass der Fokus auf die Nutzenden erhalten bleibt und dieser Fokus auch für die nächsten Planungsschritte maßgeblich ist (vgl. Schaubild 7). Auf der Grundlage dieses Themengerüstes, das über die Diskussion typischer „Fälle“ und einschlägiger Ergebnisse aus Sozialplanung und Sozialberichterstattung entwickelt wurde, wird von den am Netzwerk beteiligten Akteuren ein gemeinsames Zielsystem erarbeitet, das den Rahmen für weitere strategische und operative Planungen darstellt. Hierzu können Methoden und Instrumente des Projektmanagements genutzt werden.

Schaubild 7: Entwicklung generativer Themen und eines Zielsystems

$\begin{array}{lll}\text { Fallkonstellation A } & \text { Fallkonstellation B } & \text { Fallkonstellation C } \\ \text { Zentrale Themen, } & \text { Zentrale Themen, } & \text { Zentrale Themen, } \\ \text { z.B. } & \text { Z.B. } & \text { z.B. } \\ \text { - Familiendynamik } & \text { - schlechte } & \text { - Familiendynamik } \\ \text { - Schulden } & \text { Qualifikation } & \text { - psychische } \\ \text { - schlechte } & \text { - physische } & \begin{array}{c}\text { Gesundheit } \\ \text { Qualifikation }\end{array} \\ \text { - physische } & \text { Gesundheit } & \text { - Sucht } \\ \text { Gesundheit } & & \\ & & \end{array}$

\section{Auswahl zentraler konstellationsübergreifender Themen}

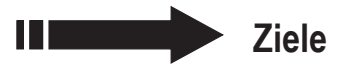

(Eigene Darstellung) 
Wenn im Netzwerk ein konkretes gemeinsames Zielsystem erarbeitet wurde, können im nächsten Schritt zur Zielerreichung Aktivitäten abgeleitet werden, an denen die jeweils zuständigen Netzwerkakteure beteiligt sind. Hierdurch wird netzwerkintern deren Zusammenhang gefestigt, die konkrete Zusammenarbeit erprobt, Kenntnisse über Schnittstellen werden erworben und Ansätze zu ihrer Überwindung können entwickelt werden. Das Netzwerk wächst über Erarbeitung von Produkten und die Gestaltung von Prozessen. Mögliche Produkte können sein: Veranstaltungen, Angebote, Leitfäden, Handbücher, Flyer, ein gemeinsamer Internetauftritt, aber auch Kooperationsvereinbarungen.

Weitergehend als die Erarbeitung einzelner Produkte ist die Gestaltung von Dienstleistungsketten, die die Leistungen verschiedener Organisationen systematisch miteinander verknüpfen und vorhandene Schnittstellen durch Schnittstellenkonzepte überbrücken. Hinter dieser Konzeption steht die Idee der Prozessorganisation. Als Gegenstück zur hierarchischen Aufbauorganisation stellt die Prozessorganisation die Abläufe in und zwischen Organisationen in den Mittelpunkt der Betrachtung und ermöglicht eine ganzheitliche und horizontale Sichtweise.

$\mathrm{Zu}$ den Kernelementen der Prozessorganisation gehört:

- Die konsequente Orientierung an Anforderungen und Ergebnissen. Jede Planung oder Neugestaltung von Leistungsprozessen beginnt bei den Anforderungen der Hilfeberechtigten; den Endpunkt stellen die erwünschten Ergebnisse dar. Dadurch wird die Sichtweise der Hilfeberechtigten auf die Prozesse in den Mittelpunkt gerückt. Die Orientierung an Anforderungen und Ergebnissen für die Hilfeberechtigten ermöglicht gerade bei der Gestaltung von Abläufen, die sich über mehrere Organisationen hinweg erstrecken, die Formulierung und Gestaltung gemeinsamer, abgestimmter Vorgehensweisen.

- Die Koordination der Prozesse. Es muss definiert werden, welche funktional getrennten, aber zusammengehörigen Leistungen miteinander zu einem Leistungsprozess verknüpft werden können. Übergänge an den Schnittstellen werden definiert und so Brüche, Parallel- und Blindleistungen reduziert. Sind die bisher isolierten Einzelleistungen durch die horizontale Betrachtung über Stellen, Abteilungen oder Einrichtungsgrenzen hinweg miteinander verknüpft, ergibt sich ein neuer $\mathrm{Zu}$ sammenhang, der im Ideal für die Hilfeberechtigten effektiver und für die beteiligten Organisationen effizienter organisiert werden kann. Ein Leitsatz von Prozessorganisationen lautet: Die Konzentration liegt auf den Nutzen generierenden Leistungsprozessen. Diese stehen im Fokus, 
denn sie ermöglichen die Erfüllung der Anforderungen bzw. der gewünschten Ergebnisse.

- Die kontinuierliche Verbesserung der Prozesse. Nach der Beschreibung und Festlegung der Dienstleistungsketten sowie der Durchführung der Aktivitäten ermöglicht die Prozessorganisation die regelhafte Überprüfung der Prozessqualität. Werden die Ziele erreicht? Wie zufrieden sind die Hilfeberechtigten? Wo zeigen sich aus der Sicht der beteiligten Organisationen Probleme bei den Einzelleistungen? Werden die richtigen Leistungen erbracht, gibt es Versorgungslücken oder Doppelleistungen? Wie funktionieren die Schnittstellen? Diese und alle weiteren interessierenden Fragen müssen mit allen beteiligten Organisationen gemeinsam beantwortet werden. Die Prozessbeschreibungen in den Dienstleistungsketten liefern für die Kommunikation die notwendige Grundlage. Das Ergebnis der Analyse ist die Basis für eine Anpassung, die die gemeinsame Planung der Prozesse auf der nächsten Stufe ermöglicht.

Über das Konzept der Dienstleistungsketten wird die Einzelfallarbeit (z. B. Case Management) mit der Planung von Infrastruktur und Leistungsangeboten systematisch verknüpft.

\subsection{Gelingensbedingungen für die Gestaltung multilateraler Kooperationsstrukturen in Produktionsnetzwerken}

Die hier dargestellten theoretischen Zugänge haben den Blick auf unterschiedliche Aspekte von Netzwerken eröffnet. In der Zusammenschau lassen sich zentrale Merkmale von Netzwerken festhalten. Gleichzeitig lassen sich aus diesem Überblick „Gelingensbedingungen“ für die Konstitution und Verstetigung von Netzwerken ableiten, die insbesondere für Netzwerke mit einem hohen Grad an verbindlicher Zusammenarbeit gelten (vgl. Abschnitt 4.2). Dadurch werden analytische Aussagen in normative Gestaltungsvorschläge transformiert. Allerdings darf nicht vergessen werden, dass „jedes Netzwerk anders“ ist, und deshalb der zentrale „Erfolgsfaktor“ der reflektierende Blick auf die Besonderheiten von Zusammensetzung und Dynamik des jeweiligen Netzwerks ist.

Zusammenfassend kann festgehalten werden:

- Wichtig ist die Einbindung aller relevanten (Gruppen von) Akteure. Im Zusammenhang mit dem Thema „Einwanderung" sind dies neben den migrationspolitischen Akteuren (Ausländerbehörde, Sozialamt, ggf. BAMF, Beratungsstellen) die zentralen Akteure im Feld sozialer Hilfen 
(Jugendamt, Gesundheitsamt, Jobcenter, freie Träger etc.). Ebenso sollten Ehrenamtliche und die Zielgruppe in geeigneter Form beteiligt werden. Bei der Konstitution von Netzwerken ist es deshalb wichtig, eine gründliche Beteiligtenanalyse vorzuschalten.

- Netzwerke benötigen gemeinsam geteilte Ziele von der Netzwerkgründung bis zur Verstetigung, diese spielen in jeder Phase der Netzwerkarbeit eine tragende Rolle. Sie sind Resultat eines kontinuierlichen Aushandlungsprozesses, der auf spezifischen rechtlichen und strategischen Zielstellungen der Akteure des Netzwerks basiert und somit eine Orientierungsmarke für eine langfristige gemeinsame Perspektive darstellt. Gemeinsame Ziele bilden die „diskursive Klammer" für die am Netzwerk beteiligten Akteure und sollten deshalb schriftlich fixiert werden. Je konkreter die Ziele formuliert sind, umso eher sind sie für gemeinsame Planungen geeignet (z. B. über die Herausbildung eines Zielsystems). Vage formulierte Ziele erleichtern zwar Kompromisse innerhalb konflikthafter Beziehungen, bergen aber die Gefahr geringer Verbindlichkeit in sich.

- Netzwerke werden stabil durch die Verbindlichkeit und Verlässlichkeit der Arbeit. Vereinbarungen über Ziele und die daraus resultierenden Aufgaben aller Akteure müssen eine verbindliche Richtschnur der Netzwerkarbeit sein. Auch bei teilweise konflikthaften Konstellationen basiert die Zusammenarbeit darauf, das Vereinbarte aus einer gemeinsamen Selbstverpflichtung zu erfüllen. Die gemeinsame Verantwortung für grundsätzliche Entscheidungen im Netzwerk ist eine wesentliche Arbeitsgrundlage für ein nachhaltig wirksames und von allen Beteiligten getragenes Netzwerk. Grundlage dieser Gemeinsamkeit können ein Wertekonsens, aber auch wechselseitige Vorteile für die Beteiligten sein.

- Verbindlichkeit setzt die Existenz und Akzeptanz von Netzwerkregeln voraus. Sinnbildlich für den besonderen Charakter von Netzwerkarbeit ist es, dass die Akteure sich selbst eigene (formelle oder informelle) Regeln geben (müssen), die nicht von einer einzelnen Organisation dominiert werden. Gleichwohl sollte die Einhaltung der Regeln gemeinsam überwacht werden, um gravierende Regelverstöße gemeinsam analysieren zu können.

- In Produktionsnetzwerken bedarf es einer klaren Aufgabendefinition und -verteilung. Netzwerkarbeit ist für viele Beteiligte Zusatzarbeit. Daher ist es vielerorts eine Frage der Organisation und Aufgabenverteilung, ob ein Netzwerk existieren kann oder nicht. Aus der Entwicklung von Zielsystemen resultieren Aufgaben, die klar und gezielt verteilt 
werden müssen, um die vielfältigen Kompetenzen angemessen nutzen zu können und (meist knappe) Ressourcen nicht durch Parallelarbeiten zu verschwenden.

- Stabilität stiften auch klare Entscheidungsstrukturen und eine breite Beteiligung an der Entscheidungsfindung. Verbindlichkeit ist nur dann gewährleistet, wenn alle am Netzwerk beteiligten (Gruppen von) Akteure an der Entscheidungsfindung partizipieren. Hierzu ist es nötig, gemeinsam Entscheidungsregeln und -wege festzulegen und schriftlich zu fixieren.

- Netzwerke konstituieren sich als soziale Systeme durch eine eigene Sinn- und Wertestruktur. Basis der gemeinsamen Arbeit ist die Entwicklung einer von allen Beteiligten geteilten Sinnstruktur, d. h. einer „gemeinsamen Sicht auf die Dinge“. Diese muss/kann nicht für alle Beteiligten identisch sein, aber ein Grundverständnis über den Gegenstand der Netzwerkarbeit, die zu verfolgenden Ziele und die Erfolgskriterien ist Voraussetzung für gemeinsame Arbeit. Damit verbunden bzw. darauf aufbauend sollte an einer „Netzwerkethik“ als Basis einer gemeinsamen Wertestruktur gearbeitet werden.

- Es gibt verschiedene Wege, solche Sinn- und Wertestrukturen zu entwickeln. Eine Möglichkeit ist die gemeinsame Rekonstruktion und Analyse von Einzelfällen (je nach Feld sind das Personen, aber auch Dinge, z. B. Gebäude), über die es gelingen kann, organisations- bzw. professionsspezifische Sinnstrukturen und "Sichtweisen“ abzugleichen und gegenseitig „anzureichern“.

- Unabdingbar ist die Erhaltung der Autonomie der einzelnen Akteure. Ein Netzwerk bildet ein soziales System mit eigenen Sinn- und Wertstrukturen, Entscheidungswegen und Regeln, dennoch darf die organisationale Autonomie der Beteiligten nicht zu stark beeinträchtigt werden. Dies beinhaltet die Berücksichtigung der Zielvorstellungen aller am Netzwerk Beteiligten (deshalb ist eine offen geführte Diskussion über Interessen und Werte sehr wichtig) und setzt sich bei der Festlegung von Regeln fort. Jenseits der Teilnahme am Netzwerk müssen die einzelnen Akteure immer autonom handeln können. Ist die Netzwerkarbeit und die dieser zu Grunde liegende Zielsetzung nicht vereinbar mit dem Selbstverständnis der Einzelorganisation, so besteht die Gefahr, dass diese das Netzwerk verlässt. Hier liegt ein grundlegendes Spannungsverhältnis zwischen Netzwerk und beteiligten Organisationen vor.

- Motor von Netzwerken ist das persönliche Engagement der Akteure. Es liegt an den einzelnen Beteiligten, mit welcher Intensität das Netzwerk 
(mit-)gestaltet wird. Gerade in den Phasen nach der Konstitution eines Netzwerks, in denen schwierige Abstimmungsprozesse stattfinden müssen, sind alle Beteiligten aufgefordert, mit persönlichem Engagement die Arbeit voranzubringen. Dies bedeutet aber, dass die Arbeit im Netzwerk so gestaltet wird, dass die Motivation zur Mitarbeit erhöht und nicht minimiert wird.

- Konkrete Ergebnisse, „schnelle Erfolge“ und „Erlebnisse“ stabilisieren Engagement und Motivation. Für alle am Netzwerk Beteiligten ist die gemeinsame Arbeit an konkreten Produkten - wie Flyer, Fachtage, Fortbildungen oder neue Angebote - wesentlich, um einerseits den Prozess der Integration voranzutreiben und andererseits der Netzwerkarbeit ein konkretes Gesicht zu geben sowie Kooperation der Netzwerkbeteiligten zu erproben und zu stärken. „Schnelle Erfolge“ durch gut umsetzbare Produkte mit einer großen Außenwirkung wie z. B. ein gemeinsamer Flyer, eine Fachveranstaltung oder ein in seiner Umsetzung transparenter „Arbeitsplan“ mit den damit verbundenen „Erlebnissen“ einer gegenüber der Alltagsarbeit hervorgehobenen Aktivität steigern die Motivation, sich im Netzwerk zu engagieren. Der „psychologische Kontrakt“ kann dadurch gezielt gestärkt werden.

- Sinnvoll ist die Anbindung an bestehende Netzwerke. Die Stabilität von Netzwerken ist eng mit ihrer Anerkennung in der lokalen Landschaft verbunden. Es ist dringend erforderlich, die bereits bestehenden Strukturen zu kennen, um sie nicht unberücksichtigt zu lassen oder gar zu übergehen. Wenn allerdings eine Anbindung an bestehende Strukturen erfolgt, muss darauf geachtet werden, dass die Ziele und Arbeitsweise des „neuen“ Netzwerks dadurch nicht verloren gehen.

- Der Nutzen des Netzwerks für die einzelnen Akteure muss immer wieder sichtbar gemacht werden können. Für die Beteiligten muss sich regelmäßig der Nutzen für die eigene Praxis erschließen, sonst können sie nicht über lange Zeit im Netzwerk gehalten werden (Vorteilsübersetzung). Der Nutzen kann unterschiedlich aussehen: Erleichterung für die alltägliche Arbeit im Sinne fallbezogen besser abgestimmter Abläufe, Verbesserung der Situation für die eigene Klientel oder Weiterentwicklung organisationsinterner Leistungsprozesse.

- Organisationale Netzwerke stützen sich auf die Mitwirkung von Entscheidungsträgerinnen und -trägern und der „operativen Ebene“. Um die Netzwerkarbeit auf Dauer stellen zu können, dürfen sich die Regelungen und Abstimmungen nicht darauf beschränken, dass die Akteure fallbezogen besser miteinander kooperieren. Um grundlegendere Regelungen zu treffen, Arbeitsabläufe verbindlich und fallübergreifend ab- 
zustimmen, braucht es die Zustimmung der jeweiligen Leitungsebene der beteiligten Organisationen. Innovative Ideen auf der operativen Ebene können nur dann dauerhaft implementiert werden, wenn auch die Entscheidungsebene in den Prozess eingebunden ist. Allerdings gilt auch umgekehrt, dass Entscheidungen auf Leitungsebene von der operativen Ebene adäquat umgesetzt werden müssen. Dies kann nicht alleine auf dem Weg hierarchischer Weisungen geschehen, vielmehr bedarf es der Akzeptanz von Zusammenarbeit durch die betroffenen Mitarbeitenden.

- An der Grenze zu den beteiligten Organisationen muss in diese hinein Überzeugungsarbeit geleistet werden. Um eine dauerhafte Strukturund Ablaufgestaltung gewährleisten zu können, müssen die innovativen Ideen des Netzwerks in die Organisationen zurückgespiegelt werden, aus denen die Akteure des Netzwerks kommen. Nur wenn die Netzwerkarbeit in den regulären organisatorischen Abläufen einen entsprechenden „Widerhall“ findet, können in den einzelnen Organisationen Strukturen geschaffen werden, die nachhaltig neue Abläufe ermöglichen.

- Last but not least: Ein Netzwerk als eigenständiges soziales System braucht eine eigene Steuerungsstruktur und ein spezielles Management inklusive Moderation, um diese Strukturen aufzubauen und umzusetzen (vgl. Abschnitt 4.8). Damit wird deutlich, dass Netzwerke bestehende Machtstrukturen tangieren und gleichzeitig selbst auf Machtstrukturen aufbauen. Die Fragen, wie eine Steuerungsstruktur aussieht und wer für das Netzwerkmanagement verantwortlich ist, sind ohne eine Reflexion der vorhandenen kommunalen Machtstrukturen (z. B. des Verhältnisses der Parteien im Kommunalparlament zueinander, die Bedeutung verschiedener Ämter und ihr Verhältnis zueinander, die Rolle der örtlichen Wirtschaft, die Rolle der Verbände, des Ehrenamts etc.) nicht hinreichend, d. h. nur technokratisch verkürzt zu beantworten. Jedes Netzwerk kann nur auf der Basis einer Analyse des „kommunalen Feldes" verstanden werden.

\subsection{Methodisches I: Wie werden „Produktionsnetzwerke“ geknüpft?}

Die oben vorgestellten theoretischen und konzeptionellen Überlegungen wurden vom Team des Instituts für Stadt- und Regionalentwicklung für die Konzipierung und Durchführung der wissenschaftlichen Begleitung des Modellprojekts „Einwanderung gestalten NRW“ genutzt. In der Aus- 
einandersetzung mit der Praxis wurde dabei ein Konzept zur Entwicklung von „Produktionsnetzwerken“ umgesetzt und erprobt. Es baut auf folgenden Grundannahmen auf:

- Komplexe Problemlagen erfordern komplexe Handlungsstrategien. Diese können nicht nur von einer Organisation (Jobcenter, Jugendamt, freier Träger) entworfen werden, sondern erfordern die gemeinsame Planung und ein koordiniertes Vorgehen mehrerer Akteure.

- Wenn es bereits institutionalisierte Formen der Handlungskoordination gibt (z. B. bereits vor Ort existierende Netzwerke), stellt das Konzept Produktionsnetzwerk eine bestimmte Arbeitsweise dar, die von den Akteuren im schon bestehenden Netzwerk umgesetzt wird. Gibt es solche Formen noch nicht, ist es Bestandteil der Netzwerkarbeit, die kooperativen Arbeitsstrukturen zu institutionalisieren, ein Produktionsnetzwerk als Form der Zusammenarbeit mehrerer Akteure aufzubauen.

- Die Problemlagen, mit denen die jeweiligen Zielgruppen von Produktionsnetzwerken konfrontiert sind, sind individualisiert, betreffen konkrete Personen und werden von diesen subjektiv verarbeitet und (teilweise auch nicht) bewältigt. Gleichzeitig sind sie typisch, gelten auch überindividuell und weisen somit strukturelle Merkmale auf. Dieses Spannungsverhältnis zwischen der allgemeinen Struktur und der Besonderheit des Einzelnen manifestiert sich im „(Einzel-)Fall“.

- Die gesetzliche Aufgabe von Helferorganisationen (Jobcenter, Jugendamt etc.) ist es, einerseits nach rechtlichen Vorschriften und damit verallgemeinerbar Unterstützung zu gewähren, diese aber an der Besonderheit des Einzelfalls auszurichten (besonders deutlich wird dies im Individualisierungsprinzip des SGB XII).

- Deshalb ist der Einzelfall besonders gut als Ausgangspunkt der Zusammenarbeit von Akteuren im Produktionsnetzwerk geeignet. Es gilt, in einem gemeinsamen Entwicklungsprozess die strukturellen Elemente der Einzelfälle herauszuarbeiten und Bearbeitungsformen zu entwerfen. Diese können dann in der Einzelfallarbeit genutzt werden.

Auf der Basis dieser Grundannahmen wurde ein methodisches Vorgehen zum Aufbau von Produktionsnetzwerken entwickelt, das sich in vier grundlegende Handlungsschritte gliedern lässt, die jeweils Bearbeitungskomplexe darstellen:

1. Vorentscheidungen und Vorbereitungen: Festlegung der initiierenden Organisation, interne Installation des Projekts, Benennung der Netzwerkkoordinatorin bzw. des Netzwerkkoordinators, Fokussierung der Zielgruppe und des Sozialraums, Sondierung und Vorbereitung von beteiligten Organisationen im Sozialraum, Zusammenstellung des 
Netzwerks, interner Auftaktworkshop, erster Netzwerkworkshop (vgl. Abschnitt 4.6.1).

2. Durchführung von Planungskonferenzen: Integrierte Fallrekonstruktion, generative Themen, Entwicklung eines Zielsystems (vgl. Abschnitt 4.6.2).

3. Umsetzung des Zielsystems durch Produkte wie Zwischenworkshops, Fallkonferenzen, gemeinsam geplante Angebote, Angebotsüberblick, Entwicklungsprojekte, systematischer Informations- und Erfahrungsaustausch oder Kooperationsvereinbarungen sowie Prozesse, z. B. Standards und Verfahren, Dienstleistungsketten oder Schnittstellenregelungen (vgl. Abschnitt 4.6.3).

4. Überprüfung und Verstetigung: Regeln und Vereinbarungen, Monitoring, Überprüfung der Arbeitsprogramme, Dokumentation, Prüfung der Dienstleistungsketten in der Fallarbeit und Reflexion (vgl. Abschnitt 4.6.4).

Im Folgenden werden die Handlungsschritte ausführlich dargestellt. Zu beachten ist aber, dass in der praktischen Umsetzung immer wieder Varianten dieser idealtypischen Vorgehensweise sowie „Schleifen“ und Redundanzen festzustellen sind, die keine Abweichung darstellen, sondern aus den Besonderheiten der Arbeitsfelder und/oder der beteiligten Organisationen resultieren.

\subsubsection{Vorentscheidungen und Vorbereitungen}

\subsubsection{Konzept}

Der erste Impuls für die Initiierung eines Produktionsnetzwerks geht von derjenigen Organisation aus, die den Bedarf nach abgestimmten Dienstleistungen für eine Zielgruppe erkannt hat und die für das Themenfeld eine gesetzliche und fachlich breit angelegte Zuständigkeit und damit eine führende Rolle hat. Kommunalverwaltungen haben als Regelversorger eine entsprechende Stellung im Feld und verfügen eher über Ressourcen, die den Aufbau eines Netzwerks ermöglichen. Baut ein freier Träger diese Art der Vernetzung auf, kann dies nur gelingen, wenn man auf bereits gewachsene Kooperationserfahrungen und -strukturen aufsetzen kann und keine unmittelbaren Eigeninteressen mit der Vernetzung verbunden sind. Denkbar ist auch, dass die Initiative von einem Kernnetzwerk von Akteuren ausgeht, die sich durch Aktivitäten in einem anderen Netzwerk bereits 
kennen oder die Notwendigkeit übergeordneter Steuerung aus den Erfahrungen gemeinsamer Arbeit in Einzelfällen erkannt haben.

Das Handlungskonzept Produktionsnetzwerke führt in der Regel auch zu Veränderungen von organisationalen Strukturen und Prozessen und stellt eine Investition in die Organisation wie in die fachliche Konzeption dar. Die eher mittelfristig angelegte Änderungsstrategie ist nur mit einem entsprechend hohen Aufwand erfolgreich zu bewerkstelligen. Daher ist die klare fachliche und politische Entscheidung in der initiierenden Organisation für das Handlungskonzept eine wesentliche Voraussetzung. In der Organisation muss zu Beginn nicht nur geklärt werden, welche Ziele mit dem Produktionsnetzwerk erreicht werden, mit welchen Partnern und welchen Methoden diese erreicht werden sollen, sondern auch ob der Bedarf an Zeit und Ressourcen zur Verfügung stehen.

Eine Vorentscheidung mit großer Tragweite ist die Benennung der Netzwerkkoordinatorin/ des Netzwerkkoordinators. Netzwerkkoordination braucht eine angemessene Ressourcen- und Zeitausstattung für Vorbereitung und Steuerung des Netzwerks, Kontaktpflege, Umsetzung von Arbeitspaketen sowie Dokumentation, eigene Weiterbildung und kollegialen Austausch und Reflexion mit anderen Netzwerkkoordinatorinnen und -koordinatoren. Entscheidend sind ebenfalls klare Befugnisse und die unmittelbare Anbindung an die oberste Leitungsebene ihrer Organisation und die stabile und kontinuierliche Begleitung sowie Rückendeckung. Zum einen um in die Organisation hinein die Bedeutung der Netzwerkarbeit zu signalisieren, zum anderen um aus der Netzwerkarbeit Entscheidungen mit strategischem oder politischem Charakter ggf. direkt abstimmen zu können. Neben fachlichen Kompetenzen und Wissen über die Versorgungslandschaft vor Ort brauchen Koordinierende mit Kompetenzen in Moderation und Projektmanagement. Der „doppelte Bezugsrahmen“ (vgl. 4.3.1.1) gilt in ganz entscheidender Weise auch für sie. Es gehört zu ihrem Handwerkszeug sich „ortskundig“ über die Aufträge, Problemsichten und Ziele der am Netzwerk beteiligten Organisationen zu machen, um aus diesem Wissen heraus in ihrer Rolle eine offene und neutrale Koordination gewährleisten zu können. Ihr wichtigstes „Kapital“ ist ihre Neutralität, die den Koordinierenden die Anerkennung und das Vertrauen ihrer eigenen Organisation wie auch das der Netzwerkakteure sichert. Die Erfahrungen der Erprobung zeigten, dass eine Aufteilung von Projektleitung, System- und Prozesskoordination sehr funktional ist. Damit ist einerseits der kollegiale Austausch gesichert, zum anderen wird eine klare Rollentrennung ermöglicht. Um die Neutralität der Netzwerkkoordination zu stärken, ist es sinnvoll, wenn die koordinierende Organi- 
sation im Netzwerk mit weiteren Vertreterinnen und Vertretern der Leitungs- und operativen Ebene vertreten ist.

$\mathrm{Zu}$ den Vorarbeiten des Produktionsnetzwerkes gehört auch das Aufgabenfeld, die Region und die Zielgruppe genau zu benennen und handhabbar einzugrenzen. Ein Netzwerk kann sich auf das gesamte Stadtgebiet oder einen bestimmten Sozialraum beziehen. Gerade bei Zielgruppen mit einer großen Streuung der Problemlagen ist die Verständigung darüber, mit welcher Subgruppe bzw. Teilproblematiken sich das Netzwerk beschäftigen soll, eine wesentliche Voraussetzung, um in die gemeinsame Arbeit einzusteigen. Auf der anderen Seite sind zu enge Eingrenzungen ebenfalls dysfunktional. Bspw. können bei Personen mit psychischer Erkrankung häufig Suchtproblematiken nicht ausgeklammert werden. Die Eingrenzung von Zielgruppe und Sozialraum ist zuerst fachlich motiviert, aber auch Basis für die Entscheidung der personellen Zusammensetzung des Netzwerks. Hilfreiche Grundlagen für diese Eingrenzungen bieten Sozialstrukturdaten sowie Kenntnisse über die Angebote und Projekte in den Sozialräumen.

Zur Klärung dienen folgende Fragen:

- Wie lässt sich die Zielgruppe genau beschreiben?

- Welche Themen stehen im Mittelpunkt?

- Welcher (Sozial-)Raum ist die Bezugsgröße für das Netzwerk?

Im Vorfeld sind auch die standortspezifischen Bedingungen abzuklären: Welche Rahmenbedingungen liegen vor, wer sind die zentralen Akteure, wo liegen (alte) Konfliktfelder, welche früheren oder parallelen Projekte gibt es, die für das Produktionsnetzwerk Bedeutung haben können? Ziel ist es, darauf vorbereitet und aufmerksam für Möglichkeiten und Restriktionen aus der Einbettung des Projekts zu sein.

Durch eine interne Auftaktveranstaltung wird das Produktionsnetzwerk innerhalb der Organisation verortet. Ziele und Vorgehen werden erläutert und die Rolle und Anbindung der Koordinierenden von Geschäftsleitungs- oder Amtsleitungsebene vorgestellt, um dem Netzwerk intern Bedeutung zu geben und damit in der Hierarchie zu installieren. Gleichzeitig dient die Auftaktveranstaltung der Bekanntmachung nach innen und zur Grundierung des Projekts auf der Praxiserfahrung der operativen Ebene. Die Veranstaltung sollte Workshopcharakter haben, so dass die operativen Fachkräfte die Gelegenheit haben, ihre Fachkenntnisse und Hinweise einfließen zu lassen und Wünsche und Anforderungen an das Projekt zu formulieren.

Zur personellen Zusammenstellung des Netzwerkes ist eine Sondierung und umfassende Vorbereitung von beteiligten Organisationen im Sozial- 
raum im Vorfeld der Auftaktveranstaltung des Produktionsnetzwerkes zielführend. Hierzu kontaktiert die initiierende Organisation die Akteure, die zwingend miteinander kooperieren sollten. In einem ersten bilateralen Gespräch werden der Anlass und das Anliegen vermittelt, das Konzept der Produktionsnetzwerke erläutert und ein gemeinsamer „Fahrplan“ entworfen. Der Kontakt zu den Netzwerkpartnern muss auf höchster Hierarchieebene erfolgen, um im Vorfeld die folgenden Anforderungen an eine Teilnahme zu erfüllen:

- Verbindlichkeit der Mitarbeit,

- Teilnahme von Vertretenden mit Entscheidungskompetenz sowie Vertretenden aus der Praxisebene,

- Übernahme von Aufgaben auch außerhalb der Netzwerktreffen sowie

- Wissen um mögliche Folgen für interne Prozesse und Strukturen in der jeweils eigenen Organisation.

Das wichtigste Ziel der Vortreffen ist, ein gemeinsames Einverständnis und die Zustimmung der Hierarchie zu einem koordinierten Vorgehen und $\mathrm{zu}$ gemeinsamer Planung für die Zielgruppe herzustellen und den wechselseitigen Nutzen der Netzwerkarbeit für die Bewältigung der operativen Arbeit in allen beteiligten Organisationen abzuklären. Nur wenn zumindest mittelfristig ein unmittelbarer Nutzen für die Kernaufgaben der am Produktionsnetzwerk beteiligten Organisationen ersichtlich wird, wird deren Beteiligung längerfristig sichergestellt sein. Ein wichtiger Maßstab für Organisationen ist dabei die Reziprozität. In das Netzwerk investierte Ressourcen wie Zeit, Geld oder auch Veränderungsbereitschaft sollen von den anderen Akteuren in ähnlichem Umfang eingebracht werden und zudem in absehbarer Zeit Früchte tragen. Durch diese Gegenseitigkeit entstehen Beziehungen und gegenseitiges Vertrauen.

Im Nachgang der Sondierungsgespräche ist für die initiierende Organisation eine Beteiligtenanalyse nützlich, mit der die Interessen und Ressourcen, aber auch Widerstände potenzieller Netzwerkpartner systematisch analysiert werden. Da Produktionsnetzwerke ein Handlungskonzept darstellen, kann alternativ zur Gründung eines neuen Netzwerkes geprüft werden, ob ein bestehendes Netzwerk in diesem Sozialraum und für die Zielgruppe die spezifische Arbeitsform als Erweiterung seines bisherigen Wirkens einführen will. Hierzu ist eine eingehende Prüfung der Netzwerklandschaft im Sozialraum notwendig.

Die Vorarbeiten schließen mit dem ersten Netzwerkworkshop ab, zu dem die Leitungskräfte der beteiligten Organisationen sowie die Vertretenden der nachgeordneten Ebenen eingeladen werden. Im ersten Workshop müssen über das Handlungskonzept und die Vorgehensweise gemeinsam 
Einvernehmen erzielt und die Rolle und Aufgaben der Akteure im Netzwerk geklärt werden. Diese sind einerseits Vertreterinnen und Vertreter ihrer Organisationen und bringen somit spezifisches Fach- und Handlungswissen sowie die partikularen Sichtweisen ein. Zum anderen sind sie im Netzwerk dafür (mit-)verantwortlich, dass alternative Herangehensweisen entwickelt und erprobt werden können. Von Seiten Ihrer jeweiligen Organisationen aus müssen die Akteure demnach mit Entscheidungskompetenzen ausgestattet sein oder zumindest einen direkten Zugang zu Entscheidungsträgern haben.

$\mathrm{Zu}$ Beginn zu klärende organisatorische Fragen sind:

- In welcher Weise treffen sich die Akteure?

- Wo und in welchem Rahmen finden die Treffen statt?

- In welchem Abstand und/oder bei welchen Anlässen finden Netzwerktreffen mit welchen Akteuren statt?

Die Netzwerkakteure müssen sich regelmäßig treffen, um ihre inhaltliche Arbeit abzusprechen. Gestaltung, Häufigkeit und Dauer dieser Treffen richten sich einerseits nach deren Inhalt und Ziel und andererseits nach den Bedürfnissen und Möglichkeiten der Akteure. Dies in eine Balance zu bringen und immer wieder für ausreichende zeitliche Ressourcen zu werben, ist Aufgabe der Netzwerkkoordination. Weitere Regeln der Netzwerkarbeit betreffen Vertraulichkeit sowie Datenschutz; es bietet sich an, diese im ersten Treffen verbindlich zu vereinbaren oder zumindest zur Diskussion zu stellen.

Die Zielgruppe und der Sozialraum, für die im Produktionsnetzwerk abgestimmte Verfahren und Vorgehensweisen erarbeitet werden sollen, sowie die dazu notwendigen Akteure können im ersten Workshop mit allen Netzwerkakteuren abschließend definiert und ggf. neu eingegrenzt werden. Dies kann per Zuruf oder Kartenabfrage geschehen. Wenn das Netzwerk sehr groß ist und/oder eine komplexere Struktur mit Arbeitsgruppen aufweist, kann eine Steuerungsgruppe gebildet werden. Steuerungsgruppen ermöglichen die Abstimmung von Ergebnissen innerhalb des Netzwerks wie auch in die Leitungsebene der beteiligten Organisationen. Eine Steuerungsgruppe muss mit Personen besetzt sein, die entscheidungsbefugt sind oder zumindest Entscheidungen herbeiführen können.

Zu klärende Fragen sind:

- Wie werden Entscheidende in die Arbeit des Netzwerkes eingebunden?

- Wie wird die operative Ebene in die Arbeit eingebunden?

- Wie werden Entscheidungsprozesse zwischen diesen Ebenen organisiert und kommuniziert? 
- Gibt es Vorgaben aus einem Steuerungsgremium, die von den Netzwerkakteuren der operativen Ebene in konkrete Verabredungen und Abläufe transformiert werden?

Ein Netzwerk kann auch als Kombination eines inneren und äußeren Kreises organisiert werden. Ein „Kernnetzwerk“ von Akteuren kann ein Produktionsnetzwerk bilden und mit einem größeren Kreis von Akteuren in einem organisierten Austausch stehen („Informationsnetzwerk“, vgl. auch Abschnitt 4.2.2). Die Zuordnung der Akteure in das Kernnetzwerk oder das Informationsnetzwerk kann festgeschrieben oder je nach Thema und Phase veränderbar sein. Die dazugehörigen Entscheidungen müssen gemeinsam gefällt werden oder mindestens transparent sein. Auch eine $\mathrm{Zu}-$ ordnung der unterschiedlichen hierarchischen Ebenen ist denkbar. So kann zu Beginn der Netzwerkbildung z. B. das „Kernnetzwerk“ aus Entscheidenden Sinn machen, das im Verlauf mit Akteuren aus den operativen Ebenen (den Arbeitsebenen) erweitert oder ersetzt wird. Jede Form erfordert für die Gestaltung der Abläufe spezifische Abstimmungsregeln.

Zu klärende Fragen sind:

- Gibt es verschiedene Beteiligungsgrade von Netzwerkpartnern?

- Macht es Sinn, in einem kleinen Kernnetzwerk zu arbeiten und einen breiteren Kreis von Akteuren durch Information und Transfer an der Arbeit teilhaben zu lassen?

- Sollten so viele Partner wie möglich eingebunden sein?

Bereits in dieser frühen Phase der Netzwerkbildung sollte die Diskussion, wenn möglich, ausgehend von einer ersten Fallanalyse ${ }^{30}$ geführt werden. Dadurch werden die Perspektiven auf den „Gegenstand“ und Differenzen in den professionellen Sichtweisen im Netzwerk viel konkreter deutlich. Die erste Feststellung der Beteiligten dient somit auch dem vertieften Kennenlernen professioneller Positionen auch bereits einander zum Teil gut bekannter Akteure, ihrer Aufgaben, Rolle und Ziele. Unter Umständen schlagen diese Unterschiede auf die Auswahl möglicher Netzwerkpartner durch.

Bereits hier stellen sich die Fragen, die im Zuge der Netzwerkarbeit immer wieder auftreten werden:

- Wer von den Anwesenden ist aktiv an diesem Fall beteiligt?

- Wer hat mit welchem gesetzlichen Auftrag mit der Zielgruppe zu tun?

- Welche Problemsicht haben die Beteiligten?

- Welche spezifischen Interessenslagen ergeben sich hieraus?

30 Konzeption und Methodik der Fallarbeit werden im nächsten Abschnitt behandelt. 
- Welche Ziele haben die Beteiligten?

- Wer müsste auf jeden Fall zusätzlich beteiligt werden?

- Wer könnte zudem beteiligt sein?

Dieser Arbeitsschritt mündet in eine Identifikation aktuell fehlender wichtiger Akteure.

\subsubsection{Methoden}

Für die Zusammenstellung des Netzwerkes und für die strategische Orientierung der Koordination bieten sich verschiedene Methoden an. Die beiden vorgestellten Methoden sollten zu Beginn der Netzwerkarbeit angewendet werden; sie liefern aber auch zu späteren Zeitpunkten durchgeführt wertvolle Hinweise. Während die Kartenabfrage mehr Informationen liefert, wenn sie mit dem gesamten Netzwerk durchgeführt wird, ist die Beteiligtenanalyse ein Instrument, das die Netzwerkkoordination auch ohne weitere Akteure zur Vorbereitung und Analyse nutzen können.

\subsection{Kartenabfrage}

Um das Netzwerk auf Vollständigkeit zu prüfen und eine Einladungsliste zu erstellen, hat sich die Methode der Kartenabfrage mit anschließender Clusterbildung bewährt. Bei der Kartenabfrage werden die Teilnehmenden im Anschluss an die Fallrekonstruktion einzeln gebeten, die nach ihrer Meinung (im konkreten Fall oder generell) wesentlichen Akteure zu benennen, die in das Netzwerk einbezogen werden sollten. Die Ergebnisse werden dann geclustert. Das Verfahren des Clusterns wird als bekannt vorausgesetzt.

\subsection{Beteiligtenanalyse}

In einer Beteiligtenanalyse werden Interessen und Ressourcen, aber auch Widerstände potenzieller Netzwerkpartner systematisch analysiert. Beteiligte in einem vorhandenen oder zu gründenden Netzwerk haben in der Regel unterschiedliche Interessen und verfügen über verschiedene Ressourcen. Auch das Konfliktpotenzial oder Widerstände gegen bestimmte Kooperationen oder Vorgehensweisen sind bei jedem (möglichen) Netzwerkpartner anders. Ziel einer Beteiligtenanalyse ist es, diese Punkte zu 
analysieren und Strategien für die Einbindung der Partner zu entwickeln. Mit einer Beteiligtenanalyse können gemeinsame Ziele und die im Feld verfügbaren oder zumindest vermuteten Ressourcen erkannt und für die Gründung und Regelung eines Netzwerkes genutzt werden. Ebenso werden Hinweise auf Zielkonflikte und unlösbare Widerstände zumindest deutlich; sie können entweder die Grenzen einer Zusammenarbeit im Vorfeld zeigen oder schon in dieser frühen Phase den realistischen Rahmen für gemeinsame Ziele beschreiben.

Das Instrument sieht die systematische Erfassung wesentlicher Informationen vor.

Tabelle 1: Beteiligtenanalyse

\begin{tabular}{|l|l|l|l|l|}
\hline & & & & \\
Beteiligte(r) & $\begin{array}{l}\text { Interessen/ } \\
\text { Ziele }\end{array}$ & $\begin{array}{l}\text { Ressourcen/ } \\
\text { Potenziale } \\
\text { Bedeutung für das } \\
\text { Netzwerk } \\
\text { (Beteiligte vs. } \\
\text { Externe) }\end{array}$ & $\begin{array}{l}\text { Restriktionen/ } \\
\text { Konflikte }\end{array}$ \\
\hline & & & & \\
\hline & & & & \\
\hline
\end{tabular}

(vgl. Bundesagentur für Arbeit 2006: S. 156)

Tatsächliche oder potenzielle Partner bzw. Beteiligte, bereits aktive Kooperationen sowie „Wunschpartner“, die neu für eine Zusammenarbeit gewonnen werden sollen, werden ebenso berücksichtigt. In tabellarischer Form werden die Ziele der jeweiligen Beteiligten bzw. die im gesetzlichen Rahmen festgelegten Ziele bezüglich der Arbeit mit der Zielgruppe wie auch die Interessen und die die Motivation der Beteiligten, sich an einem Netzwerk zu beteiligen, vergegenwärtigt. Dazu gehören z. B. strategische, 
fiskalische und politische Interessenslagen. Die Ressourcen und Potenziale wie Mittel, Kompetenzen, Kenntnisse und Kontakte, die die Beteiligten in das Netzwerk einbringen könnten, werden ebenso vergegenwärtigt wie die Bedeutung des Netzwerkpartners, ob die jeweilige Organisation am Netzwerk beteiligt wird, werden soll oder will oder eine Rolle außerhalb des Netzwerks adäquater wäre (z. B. als externer Auftragnehmer). Mit Restriktionen/Konflikte sind vor allem Widerstände gegen das Netzwerk gemeint, die sich in Bedenken oder Blockadehaltung gegen andere Beteiligte äußern. „Konflikte“ können aus den oben genannten Widerständen resultieren. Die Spalte nimmt aber auch Konflikte auf, die fruchtbar für ein Netzwerk sein können: offene Zielkonflikte, aktuelle organisationsinterne Veränderungen, transparente Konkurrenzen etc.

Mit dem spezifischen Informationsbedarf können sich die Spalten des Rasters jederzeit dynamisch verändern. Die Beteiligtenanalyse ist in der Regel ein Instrument der Koordination. Aus der Zusammenschau der verschiedenen Informationen können Strategien für das Netzwerk entwickelt werden. Dazu ist nötig, die Eigenschaften und Notwendigkeiten der einzelnen Partner zu analysieren und diese im besten Fall für die Netzwerkarbeit nutzbar zu machen. Gerade auch bei schwierigen Situationen ist sie als Raster für die Analyse und Bearbeitung von Problemen oder Konflikten für die Koordination nützlich.

Grundsätzlich kann die Beteiligtenanalyse im Netzwerk durchgeführt werden und dient so der Vergegenwärtigung von Gemeinsamkeiten, Unterschieden und nützlichen Ergänzungen. Eine weitere Verwendungsweise kann darin bestehen, die Beteiligtenanalyse in regelmäßigen Abständen durchzuführen, um Veränderungen im Netzwerk aufzuspüren.

\subsubsection{Planungskonferenzen}

Das zentrale Element bei der Entwicklung und Stabilisierung von Produktionsnetzwerken sind Planungskonferenzen. Sie sind die Orte, in denen die Akteure eines Produktionsnetzwerkes gemeinsam Leistungsprozesse konzipieren und überprüfen, Schnittstellen definieren und Übergänge zwischen einzelnen Leistungen festlegen.

Planungskonferenzen basieren - wie in Abschnitt 4.4 ausführlich dargestellt - auf der gemeinsamen Rekonstruktion von „Fällen“. Diese Rekonstruktion dient nicht der Lösung dieser Einzelfälle, sondern zielt auf die Identifikation von Strukturen, die immer wieder Fallverläufe prägen und durch gemeinsame Anstrengungen der Netzwerkpartner so bearbeitet wer- 
den sollten, dass negative Auswirkungen zumindest minimiert werden. Allerdings erschöpft sich das Material für die Planungskonferenzen nicht in der Analyse typischer Einzelfälle, vielmehr wird die Beschreibung von Strukturen erleichtert durch Daten aus der Sozial- und Arbeitsmarktberichterstattung, dem Integrationsmonitoring und andere Planungsdaten. So können zur Analyse der Wohnungssituation in einem Sozialraum z. B. Daten zur sozialen Segregation, der Mietpreisspiegel, Daten zu Räumungsklagen sinnvoll ausgewertet werden, um Probleme zu identifizieren, die sich in Einzelfällen zeigen, ohne dass sie immer auftreten müssten. Eine Planungskonferenz setzt an der gemeinsamen Analyse von typischen Einzelfällen an (integrierte Fallrekonstruktion, vgl. Abschnitt 4.4). Diese mündet in der Feststellung von Themen, mit denen übergreifend über die einzelnen Fallkonstellationen Anlässe für sozialpolitische oder sozialarbeiterische Interventionen benannt werden können. Ergänzt durch Erkenntnisse aus der Sozial- und Arbeitsmarktberichterstattung bzw. dem Integrationsmonitoring (vgl. Kapitel 6) können damit „generative Themen“ identifiziert werden, mit denen aktuelle Problemsituationen prägnant und anschaulich erfasst werden können (vgl. Abschnitt 4.6.2.2). Diese generativen Themen bilden den Ausgangspunkt für die Entwicklung eines von den Netzwerkakteuren gemeinsam getragenen Zielsystems, das als Grundlage für die Planung von Aktivitäten zur Bearbeitung struktureller Probleme dient (vgl. Abschnitt 4.6.2.3).

Planungskonferenzen dürfen nicht verwechselt werden mit Fallkonferenzen, in denen es nicht um die Planung von Versorgungsstrukturen, sondern um die Bearbeitung und möglichst auch Lösung von Einzelfällen geht. Fallkonferenzen sind keine Planungsinstrumente, sondern Instrumente zur Umsetzung von Planungen (vgl. Abschnitt 4.6.3.2.2).

Die Zusammensetzung einer Planungskonferenz ist entscheidend für die Ergebnisse. In den Planungskonferenzen müssen operative Erfahrungen mit Fällen und Fallkonstellationen ebenso vertreten sein wie die Ebene, auf der (organisationale) Entscheidungen getroffen werden können. Dazu sollte die Planungsebene (Sozial- oder Jugendhilfeplanung) vertreten sein, damit die Erkenntnisse der Planungskonferenzen mit den Zahlen und Daten der Planer zusammengeführt werden können. 


\subsubsection{Erarbeitung integrierter Fallrekonstruktionen}

\subsection{Konzept}

Vor dem Hintergrund der oben skizzierten theoretischen Überlegungen werden die Teilnehmenden einer Planungskonferenz gebeten, Falldarstellungen vorzubereiten, die dann in der gemeinsamen Sitzung bearbeitet werden. Die Falldarstellungen sollen authentisch sein, es soll sich also nicht um konstruierte Fälle handeln. Konstruierte Fälle werden immer nur aus der Sicht der Fachkräfte skizziert, die „Autonomie der Lebenspraxis“ (vgl. Oevermann 2012) als Herausforderung für die Bearbeitung verschwindet hinter der Perspektive der Fallgebenden. Und es sollen typische Fälle sein, die exemplarisch die Probleme widerspiegeln, mit denen die Zielgruppe insgesamt häufig konfrontiert ist.

\subsection{Methode}

Der Fall wird geschildert, in seinen Strukturen ggf. auch visualisiert. Danach werden die Vertreterinnen und Vertreter der teilnehmenden Organisationen gebeten, sich zu folgenden Fragen zu äußern:

- Welche Rolle nehmen Sie im vorliegenden Fall ein?

- Was ist Ihr gesetzlicher Auftrag im Umgang mit diesem Fall?

- Um was geht es in diesem Fall? Skizzieren Sie die Situation aus Ihrer professionellen Sicht!

- Welche Probleme und welche Ressourcen sehen Sie im Hinblick auf die einzelnen Personen/die Familie/die Bedarfsgemeinschaft?

- Welche „Logik“ steckt Ihrer Meinung nach in dem Handeln der in den Fall involvierten Personen?

- Was wäre Ihr Ziel in der Fallbearbeitung?

- Welches Konzept verfolgen Sie im vorliegenden Fall?

Dadurch werden sowohl Aufgaben und Rollen wie auch die professionelle Sichtweise der einzelnen Akteure transparent. Im Anschluss werden die Antworten verglichen und diskutiert. Die Diskussion dient der Identifikation unterschiedlicher Sichtweisen. Außerdem können die Themen festgestellt werden, die die einzelnen Akteure mit diesem Einzelfall verknüpfen. Hier können bereits Wirkungszusammenhänge erkannt werden. Relevante Fragestellungen sind hier: 
- Was ist der Fall?

- Was ist der (gesetzliche bzw. institutionelle) Auftrag der einzelnen Akteure im Umgang mit diesem „Fall“?

- Was sind aus der Sicht der einzelnen Akteure zentrale Themen?

- Welches Ziel verfolgen die jeweiligen Akteure?

- Welche Interventionsstrategien verfolgen die einzelnen Akteure?

- Welche Wirkungen haben diese Interventionen?

Wenn die professionellen Perspektiven ausgetauscht sind und die aus ihrer Sicht relevanten Themen identifiziert wurden, wird das Arbeitsergebnis kontrastiert mit der hypothetischen Sicht der Nutzenden („Nutzerperspektive“). Diese ist insofern wichtig, als sie häufig deutlich von der der Fachkräfte abweicht und dadurch deren Perspektive als „Konstruktion“ deutlich wird. Wenn keine wirklichen Nutzenden an der Sitzung teilnehmen, muss deren Perspektive entweder von der Moderatorin/ dem Moderator oder von den Beteiligten eingenommen werden.

Folgende Fragen können die Diskussion anleiten:

- Wie sieht die Nutzerin/der Nutzer die Situation?

- Wie sieht sich die Nutzerin/der Nutzer im Verhältnis zu weiteren beteiligten Personen (z. B. Familienmitgliedern)?

- Welche Erwartungen hat die Nutzerin/der Nutzer an die Unterstützung/Angebote der verschiedenen Akteure?

- Warum agiert sie/er so, wie sie/er agiert? Was ist ihre/seine Motivation? Was ist das Ziel der Nutzerin/des Nutzers?

- Welche Handlungs- bzw. Verhaltensoptionen hat der Nutzer/die Nutzerin?

- Was hat der Nutzer/die Nutzerin bereits unternommen?

- Welche Strategien werden sichtbar?

Abschließend wird im Sinne einer Gesamtschau eine Fallkonstellation rekonstruiert, in der die einzelnen professionellen Perspektiven und die Sichtweisen der Nutzenden eingebunden sind. Dabei geht es nicht um den „kleinsten gemeinsamen Nenner“, sondern durchaus darum, Unstimmigkeiten und alternative Interpretationen festzuhalten und damit ggf. den Blickwinkel zu erweitern. Im Zentrum steht dabei der Versuch, die Fallkonstellation als Wirkungsgefüge zu rekonstruieren, d. h. zu zeigen, wie die verschiedenen Aktivitäten der Beteiligten ineinandergreifen, sich je nachdem verstärken oder neutralisieren und wie die jeweils individuellen Intentionen faktisch wirken. Häufig wird sich zeigen, dass Interventionen von Seiten der Helfenden nur dann greifen, wenn sie von einem Fallverstehen getragen sind, dass die Perspektiven aller Beteiligten (einschließlich der Adressatinnen und Adressaten) berücksichtigt. 
Mögliche Fragestellungen:

- Wie sieht das „Wirkungsgefüge“ im Fall konkret aus? Wie wirken die Leistungen der einzelnen Akteure aufeinander? Wo behindern sich Akteure in der Leistungserbringung? Wie werden Leistungen aktuell miteinander kombiniert? Wie sind Übergänge/Übergaben gestaltet?

- Verändern sich durch die Einbeziehung der unterschiedlichen Perspektiven der Nutzenden die möglichen Stellschrauben?

- Sind die Interventionen dadurch neu bzw. anders zu denken?

Das integrierte Fallverstehen mündet in einer abschließenden gemeinsamen Fallanalyse, in der die Fallkonstellation verdeutlicht wird und folgende Fragen beantwortet werden:

- Wie sieht die aktuelle Situation aus?

- Ist eine Falldynamik zu erkennen? Wenn ja, wie kann sie beschrieben werden? Von welchen Faktoren hängt die Dynamik ab?

- Wenn nein, wodurch wird die statische Situation aufrechterhalten?

Bis zu dieser Stelle ist die Arbeit am Einzelfall mit der Arbeit an der fallübergreifenden Struktur methodisch analog. Detailkenntnisse über die Person bringen eine Vertiefung von Einsichten und ein individualisiertes Fallverstehen kann stattfinden - optimalerweise als kollektiver Prozess. Die Identifikation von Themen und damit die Rekonstruktion von Strukturen, die den Einzelfall prägen, sind für Fallverstehen genauso elementar wie das Verstehen subjektiver Motive der real handelnden Personen. Diese Strukturen werden in ihrer prägenden Wirkung rekonstruiert. Ohne sich intensiv mit diesen Motiven zu beschäftigen, kann das Individuum als „Exemplar seiner Klasse“ angesehen werden, an dem hinter der individuellen Biografie strukturell geformte Lebensläufe, Problemkonstellationen sowie gesellschaftliche Bedingungen sichtbar werden. In diesem Arbeitsschritt besteht daher die Herausforderung sich explizit nicht in Falllösungen zu „verirren“, sondern gezielt Strukturen zu ergründen.

Die Falldarstellung kann auf unterschiedliche Weise realisiert werden, entweder als freie Schilderung oder unter Zuhilfenahme methodischer Instrumente wie z. B. einem Soziogramm oder einem Genogramm. Der Grad der Systematisierung ist von pragmatischen Überlegungen (z. B. Zeitressourcen) abhängig aber auch von der Bereitschaft der teilnehmenden Personen sich - wenn nicht bekannt - auf oben genannte Instrumente einzulassen. Für den weiteren Verlauf der gemeinsamen Netzwerkarbeit können auch Anforderungen an die Falldarstellungen vereinbart werden oder Formblätter entwickelt werden. 


\subsubsection{Feststellung "generativer Themen“}

\subsection{Konzept}

Die Fallkonstellation kann als ein Ensemble von gesellschaftlich, professionell oder organisatorisch gesetzten expliziten oder impliziten Themen angesehen werden, die an einem Einzelfall deutlich werden. So stellt eine im Einzelfall prekäre Wohnungssituation die Ausprägung des Themas „drohende Wohnungslosigkeit" dar und dieses Thema kann als relevantes Thema festgehalten und ggf. weiter bearbeitet werden. Auch kann das reale Verhalten einer Person als individualisierte Variante eines (wirklich oder vermeintlich) typischen Verhaltens einer ganzen Personengruppe kodiert werden und in dieser Kodierung die konkrete Bearbeitung leiten. Parallel hierzu kann die Kodierung in beiden Fällen aber als solche, d. h. als „,Zuschreibung" identifiziert werden, die mehr oder weniger triftig sein kann.

Es gilt also, diese Themen, die handlungsleitend sein können, in den einzelnen Fallkonstellationen zu erkennen. Jede Fallkonstellation ist damit als Kombination oder auch Nebeneinander unterschiedlicher Themen zu beschreiben, die für die einzelnen Beteiligten eben unterschiedlich sein können und es vermutlich auch sind (vgl. Abschnitt 4.4). Wird die Arbeit an konkreten Fällen, d. h. die Erarbeitung von „Fallkonstellationen“ mit weiteren typischen Fällen, wiederholt, können aus den Themen, die in den einzelnen Fällen identifiziert wurden, fallübergreifende Themenfelder definiert werden. Dabei stellen sich z. B. folgende Fragen:

- Welche Themenfelder wurden in den typischen Fällen identifiziert?

- Welche dieser Themenfelder sollten gemeinsam bearbeitet werden?

- Gibt es weitere Themenfelder, die in den Fällen nicht aufgetreten sind, aber gemeinsam bearbeitet werden sollten?

Für Identifikation von Strukturen ist es sinnvoll, nicht nur eine einzelne Fallkonstellation zu betrachten, sondern vielmehr eine Anzahl, die ausreicht, die Typik der Arbeit in einem Feld und damit das Typische der Fallkonstellationen zu erkennen und zu analysieren. Die Identifikation von Themen abstrahiert von den Besonderheiten des Einzelfalles und konkretisiert "Strukturen“. Strukturen gehen nicht in der Addition von Einzelfällen auf. Es handelt sich dabei vielmehr um übergreifende Zusammenhänge, die in sich einzelnen Fallkonstellationen niederschlagen können, dies aber nicht immer tun. Deshalb können diese Zusammenhänge auch unabhängig von Einzelfällen identifiziert werden, z. B. im Rahmen von Sozialund Arbeitsmarktberichterstattung oder statistischen Analysen. Sie stellen eine Realität eigenen Ursprungs dar, die in ihrem Spannungsverhältnis zu 
den Einzelfällen rekonstruiert werden muss. Die Identifikation „generativer Themen“ leitet diese Rekonstruktion an.

\subsection{Methode}

Bei jeder integrierten Fallrekonstruktion soll ein Themenspeicher erstellt werden, in dem die "generativen Themen" festgehalten werden. Nach Abschluss der integrierten Fallrekonstruktionen wird der Themenspeicher, in dem dann alle identifizierten Themen festgehalten sind, auf seine Vollständigkeit hin überprüft. Dann werden in einem zusammenfassenden Schritt die Themenfelder ausgewählt, an denen im Netzwerk weitergearbeitet werden soll. Die Themenfelder werden hierzu gemeinsam betrachtet und mit Blick auf die Relevanz für die gesamte Zielgruppe einerseits, die Notwendigkeit koordinierter Bearbeitung andererseits diskutiert. So werden ausgehend von den Fallrekonstruktionen "generative Themen“ identifiziert. Die Diskussion von Lösungen für den vorgestellten „Fall“ wird vermieden.

\subsubsection{Die Entwicklung eines Zielsystems}

\subsection{Konzept}

Aus der Bearbeitung der identifizierten generativen Themen wird in der Planungskonferenz im nächsten Schritt ein gemeinsam getragenes Zielsystem entwickelt.

Das Gelingen verbindlicher Netzwerkarbeit hängt nicht zuletzt davon ab, dass die Netzwerkakteure gemeinsame Ziele verfolgen. Damit diese Ziele nicht unspezifisch und unverbindlich bleiben, sondern tatsächlich handlungsleitend für alle Akteure im Netzwerk werden können, müssen sie systematisch gemeinsam erarbeitet werden. Deshalb ist es wichtig, Sorgfalt auf die Erarbeitung und die präzise Formulierung von Zielen zu verwenden und ein zusammenhängendes Zielsystem für die Zielgruppe zu entwickeln. In der Erarbeitung dieses Zielsystems wird Transparenz über unterschiedliche Perspektiven, gesetzliche Aufträge und Wertorientierungen geschaffen. Konsens oder auch Kompromiss sind zentrale Eckpfeiler späteren gemeinsamen Handelns. Da auf der Grundlage des Zielsystems später konkrete Aktivitäten geplant werden, die auch Ressourcen binden, 
ist es wichtig, dass das Zielsystem von allen Netzwerkpartnern getragen wird.

Das Zielsystem wird aus den identifizierten „generativen Themen“ abgeleitet. Es können unterschiedliche Methoden der Entwicklung von Zielen eingesetzt werden. Grundsätzlich ist es wichtig, dass sich die erarbeiteten gemeinsamen Ziele auf die Nutzerinnen und Nutzer beziehen, also angegeben wird, was im Hinblick auf deren Lebenssituation erreicht werden soll. Dieses Vorgehen wird durch den Bezug auf „Fallkonstellationen“, die auch die "Nutzerperspektive“ enthalten, erleichtert. In der Umsetzung sind sowohl nutzerbezogene Ziele als auch systembezogene Ziele zu formulieren. Unter Umständen werden auch Ziele für die Zusammenarbeit der Akteure im Netzwerk definiert. Dabei ist zu beachten, ob die Erreichung eines Ziels zur besseren Zusammenarbeit untereinander tatsächlich einzelnen Nutzerinnen und Nutzern bei der Bewältigung ihrer Lebenslage nutzt und damit zum Erreichen des Grundsatzziels beiträgt. Die Entwicklung von Zielen stützt sich auf Instrumente, die im Kontext von Projektplanung erarbeitet wurden.

So hat sich eine Methode bewährt, die in Planungsprozessen in unterschiedlichen Feldern seit langem eingesetzt wird und die darauf abhebt, nicht nur einzelne Ziele zu benennen, sondern ein Zielsystem zu entwickeln, das von allen Beteiligten getragen wird. Ausgangspunkt für die Entwicklung und Nutzung eines Zielsystems ist die Idee, dass Ziele eine als defizitär oder problematisch erkannte gegenwärtige Situation mit einer Vision für die Zukunft verknüpfen und so zum zentralen Instrument von Projektentwicklung und -steuerung werden. Die Entwicklung von Zielen setzt im Rahmen der Planung von Veränderungsprojekten an einer Analyse der gegenwärtigen Situation an, z. B. der Bedarfslage von Personengruppen. In diesem Kontext beinhalten Ziele die Formulierung eines wünschenswerten und anzustrebenden Zustands in der Zukunft. Aktuelles und zukünftiges Handeln der an der Zielformulierung beteiligten Akteure werden an diesem Zustand ausgerichtet. Dazu müssen die Ziele systematisiert werden. Im Projektmanagement hat sich die Unterscheidung von drei Zielebenen etabliert (dreigliedriges Zielsystem): Aus einem übergeordneten Grundsatzziel (auch Leitziel oder Oberziel genannt) werden in einem ersten Schritt untergeordnete strategische Rahmenziele (auch Mittlerziele) und in einem zweiten Schritt operative Ergebnisziele (auch Teilziele genannt) abgeleitet. Die operativen Ergebnisziele sind in konkrete Aktivitäten umsetzbar und somit unmittelbar handlungsleitend.

Die Entwicklung eines Zielsystems erfordert die aktive Teilnahme aller Beteiligten, um Kohärenz und Handlungsfähigkeit zu erzielen. Gerade in 
Netzwerken ist dies unverzichtbar, damit das Zielsystem die unterschiedlichen Perspektiven der Netzwerkakteure repräsentieren kann. Ziele sind dann steuerungsrelevant, wenn die Zielerreichung nicht nur geplant, sondern auch begleitet und kontrolliert wird; d. h. die Aktivitäten werden umgesetzt und auf Zielerreichungsgrad und Effektivität überprüft, wobei das Bewertungsergebnis Grundlage einer erneuten Zielentwicklung sein kann. Auch bei diesem Prozess ist wiederum das Mitwirken aller Akteure von Vorteil, denn nur so handelt es sich um eine kollektiv getragene Perspektive, die letztendlich die Chance auf gemeinsames Lernen eröffnet. Die gemeinsame Formulierung von Zielen kann ein „zähes Geschäft" sein. Bei der Aushandlung helfen Vorschläge von Seiten der Netzwerkkoordination oder Vorschläge aus Unterarbeitsgruppen zu bestimmten Themenbzw. Zielbereichen. Falls der Prozess z. B. durch langwierige Diskussionen ins Stocken kommt, muss das nicht nur als Hinweis auf die Schwierigkeiten der Zusammenarbeit gewertet werden, sondern verweist vor allem auf die Komplexität der erforderlichen Unterstützungsleistungen für die Zielgruppe.

Die Grundlagen für die Gestaltung eines derartigen Zielsystems stammen aus der Methodik "Zielorientiertes Planen von Projekten und Programmen der technischen Zusammenarbeit (ZOPP)“ und deren Erweiterung „Project Cycle Management (PCM) und Zielorientierte Projektplanung". Diese Planungsmethodik wird von der Gesellschaft für internationale Zusammenarbeit (GIZ, vormals GTZ) verwendet, um komplexe Planungsprozesse in der Entwicklungsarbeit transparent und nachvollziehbar zu machen und um Kontroll- und Steuerungsmöglichkeiten zu erhalten. (vgl. GTZ 1987; o. J.) Inzwischen wird Planungsmethodik sowohl in verschiedenen Bundesländern zur Programmplanung und Umsetzung als auch im Ramen der EU (in ähnlichen Varianten) verwendet. Diese treten an die Stelle der "Problemanalyse“ im beschriebenen ZOPP-Verfahren. (vgl. GTZ 1987)

\subsection{Methode}

Im Folgenden wird das Zielsystem, wie es vom Institut für Stadt- und Regionalentwicklung im Rahmen verschiedener Projekte entwickelt wurde, als Instrument vorgestellt und das Vorgehen erläutert. 
Schaubild 8: Schematische Darstellung des Zielsystems

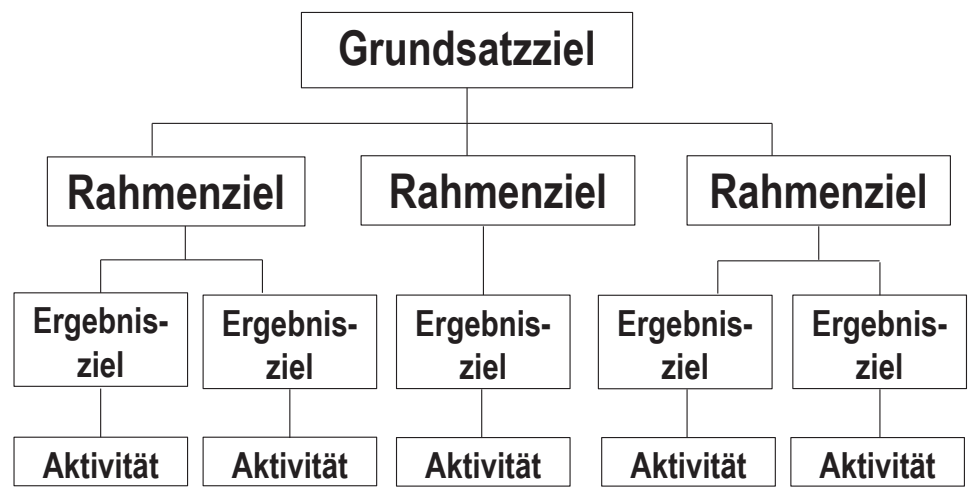

(Eigene Darstellung)

Das Zielsystem umfasst mehrere Ebenen: Vom Grundsatzziel, das den allgemeinen Ansatz kennzeichnet, bis hin zu Aktivitäten, denen ein Mengenund Kostengerüst zugeordnet ist (vgl. Schaubild 8).

Ziele sind zuerst eine Setzung (im Rahmen des Verständigungsprozesses), die von oben nach unten vorgenommen wird. D. h., jedes Ziel wird durch weitere Ziele und letztendlich durch Aktivitäten untersetzt. Das Zielsystem beinhaltet somit eine vertikale Hierarchie und ist auf seine innere Konsistenz hin zu überprüfen:

- Zuerst wird ein Grundsatzziel festgelegt, das allgemein die Verbesserung der Problemsituation benennt. Das Grundsatzziel bildet die Grundlage für die Rahmen- und Ergebnisziele und wird durch gemeinsame Programme und Projekte realisiert.

- Das Rahmenziel behandelt die jeweils konkrete Umsetzungsebene. Es beschreibt die beabsichtigten Wirkungen, also den erwarteten Nutzen, und ist ein Beitrag zum Grundsatzziel. Die Zielformulierung beinhaltet eine Verständigung aller beteiligten Akteure, die auf die lokalen Bedingungen abgestimmt ist.

- Ergebnisziele (Teilergebnisse) stellen die Zielsetzungen dar, die durch das Netzwerkmanagement erreicht werden müssen.

- Aktivitäten stellen die notwendigen Tätigkeiten zur Erreichung der Ergebnisziele dar. Sie sollten nicht zu detailliert erfasst werden (hier droht sonst Unübersichtlichkeit).

Wenn dieses Zielsystem für weitere Planungen und insbesondere für ein Monitoring verwendet werden soll, muss es um weitere Elemente ergänzt werden. 
Weitere wichtige Elemente des Zielsystems:

- Ziele sollten messbar sein. Die Messpunkte, an denen die Zielerreichung festgestellt wird, sind durch Indikatoren zu identifizieren. Diese sollten Aussagen zur Qualität, zur Quantität und zur Zeitvorstellung der Zielerreichung ermöglichen. Indikatoren dienen also dazu, den Erreichungsgrad zu überprüfen.

- Indikatoren können sich auf unterschiedliche Quellen beziehen, z. B. auf offizielle Dokumente, Statistiken, Beobachtungsprotokolle.

- Jede Planung arbeitet mit impliziten Annahmen über die Stabilität bzw. Vorhersehbarkeit der Verhältnisse, in denen geplant wird (z. B. gleichbleibende Wirtschaftssituation, prognostiziertes Bevölkerungswachstum, Finanzausstattung eines Projekts). Es ist sinnvoll, diese Annahmen explizit zu machen, um die Bedingungen anzugeben, unter denen die Planung erfolgt.

Aus der Kombination von Zielsystem, Indikatoren, Quellen und Annahmen kann folgende Planungsmatrix erstellt werden (Tabelle 3):

Tabelle 2: Planungsmatrix Zielsystem

\begin{tabular}{|c|c|c|c|c|c|c|}
\hline \multicolumn{4}{|l|}{ Grundsatzziel } & Indikator(en) & Quelle(n) & Annahme(n) \\
\hline \multicolumn{4}{|l|}{ Rahmenziel 1} & Indikator(en) & Quelle(n) & Annahme(n) \\
\hline Ergebnisziel & Indikator(en) & Quelle(n) & Annahme(n) & Aktivität(en) & \multicolumn{2}{|l|}{ Akteur(e) } \\
\hline Ergebnisziel & Indikator(en) & Quelle(n) & Annahme(n) & Aktivität(en) & Akteur(e) & \\
\hline Ergebnisziel & Indikator(en) & Quelle(n) & Annahme(n) & Aktivität(en) & Akteur(e) & \\
\hline \multicolumn{4}{|l|}{ Rahmenziel 2} & Indikator(en) & Quelle(n) & Annahme(n) \\
\hline
\end{tabular}


Alle Ziele werden als positive Zustände bzw. Eigenschaften beschrieben und zwar als wären sie schon eingetreten, als positive Ergebnisse. Zudem müssen Grundsatzziel, Rahmenziel und Ergebnisziele in sich und zueinander stimmig sein. Aktivitäten werden im Unterschied zu den Zielen als Anweisungen formuliert. Es ist zu empfehlen, eine erste Zielhierarchie als eigenständige Übersicht zu erstellen, ohne weitere detaillierte Angaben zu Indikatoren, Quellen und Annahmen zu machen. Das Rahmenziel ist auf mögliche Alternativen, also andere Möglichkeiten zur Untersetzung des Grundsatzziels zu überprüfen. Ebenso sind Alternativen zu den Ergebniszielen des Projekts zu erwägen. Dann erfolgt die endgültige Zielfestlegung nach Kriterien wie Sinnhaftigkeit und Realisierbarkeit.

Nach dieser Zielformulierung folgt die Operationalisierung - das „Kleinarbeiten“ - auf die konkreten Aktivitäten hin. Sie können in der Reihenfolge zeitlich oder auch logisch angeordnet werden. Zu Beginn der Projektplanung stellen Indikatoren vorerst Richtwerte dar, die im Verlauf eventuell angepasst und korrigiert werden müssen.

Prüfungsschritte werden im Gegensatz zur Zielformulierung von unten nach oben vollzogen. Zielformulierung bzw. Aktivitäten müssen zusammen mit den Annahmen die notwendigen und hinreichenden Bedingungen für die nächste, darüber liegende Ebene beschreiben.

\subsubsection{Ablauf einer Planungskonferenz und Entwicklung von Zielen}

Bei der Durchführung der Planungskonferenzen hilft ein klar strukturierter Ablauf (Tabelle 4). Es ist Aufgabe der Moderatorin/des Moderators und der Gruppe auf diese Struktur zu achten. Folgende Übersicht kann dabei helfen:

\begin{tabular}{|l|l|}
\hline TOPs & Wichtig! \\
\hline Phase I & $\begin{array}{l}\text { Die Fallgeberin/der Fallgeber } \\
\text { kann nicht moderieren! }\end{array}$ \\
\hline Auswahl der Moderation & $\begin{array}{l}\text { Auswahl der/des Teilnehmenden, der die Nutzerper- } \\
\text { spektive übernimmt }\end{array}$ \\
\hline $\begin{array}{l}\text { Schilderung des Falls } \\
\text { (mündlich, visualisiert, mit Genogramm) }\end{array}$ & $\begin{array}{l}\text { Keine Diskussion, keine Unter- } \\
\text { brechung durch die weiteren } \\
\text { Teilnehmerinnen/Teilnehmer }\end{array}$ \\
\hline Gelegenheit für Rückfragen zum Fall & $\begin{array}{l}\text { Verständnisfragen - keine Dis- } \\
\text { kussion, keine Lösungen! }\end{array}$ \\
\hline
\end{tabular}




\begin{tabular}{|c|c|}
\hline TOPs & Wichtig! \\
\hline $\begin{array}{l}\text { Darstellung der Perspektive jedes anwesenden Ak- } \\
\text { teurs auf den geschilderten Fall } \\
\text { - Welche Rolle nehmen Sie im vorliegenden Fall ein? } \\
\text { - Was ist Ihr gesetzlicher Auftrag im Umgang mit } \\
\text { diesem Fall? } \\
\text { - Um was geht es in diesem Fall? Skizzieren Sie die Si- } \\
\text { tuation aus Ihrer professionellen Sicht! } \\
\text { - Welche Probleme und welche Ressourcen sehen Sie } \\
\text { bei einzelnen Personen/ der Familie/ der Bedarfsge- } \\
\text { meinschaft? } \\
\text { - Welche „Logik“ steckt nach Ihrer Meinung in dem } \\
\text { Handeln der in den Fall involvierten Personen? } \\
\text { - Was wäre Ihr Ziel in der Fallbearbeitung? } \\
\text { - Welches Konzept verfolgen Sie im vorliegenden } \\
\text { „Fall“? }\end{array}$ & $\begin{array}{l}\text { Eventuell Vorbereitung der ein- } \\
\text { zelnen Akteure in Kleingruppen } \\
\text { einplanen }\end{array}$ \\
\hline $\begin{array}{l}\text { Diskussion der unterschiedlichen Perspektiven } \\
\text { - Was ist der Fall? } \\
\text { - Was ist der (gesetzliche bzw. institutionelle) Auf- } \\
\text { trag der einzelnen Akteure im Umgang mit diesem } \\
\text { Fall? } \\
\text { - Was sind aus der Sicht der einzelnen Akteure zen- } \\
\text { trale Themen? } \\
\text { - Welches Ziel verfolgen die jeweiligen Akteure? } \\
\text { - Welche Interventionsstrategien verfolgen die einzel- } \\
\text { - } \text { nen Akteure? }\end{array}$ & \\
\hline $\begin{array}{l}\text { Fragen für die Perspektive des Nutzers/der Nutzerin } \\
-\quad \text { Wie sieht die Nutzerin/der Nutzer die Situation? } \\
\text { - Wie sieht sich die Nutzerin/der Nutzer im Verhält- } \\
\text { nis zu weiteren beteiligten Personen (z. B. Familien- } \\
\text { mitgliedern)? } \\
\text { - Welche Erwartungen hat die Nutzerin/der Nutzer } \\
\text { an die Unterstützung/Angebote der verschiedenen } \\
\text { Akteure? } \\
\text { - Warum agiert sie so, wie sie agiert? Was ist ihre/ } \\
\text { seine Motivation? Was ist das Ziel der Nutzerin/des } \\
\text { Nutzers? } \\
\text { - Welche Handlungs- bzw. Verhaltensoptionen hat } \\
\text { der Nutzer/die Nutzerin? } \\
-\quad \text { Was hat der Nutzer/die Nutzerin bereits unternom- } \\
\text { men? } \\
\text { - Welche Strategien werden sichtbar? }\end{array}$ & \\
\hline
\end{tabular}




\begin{tabular}{|c|c|}
\hline TOPs & Wichtig! \\
\hline Gemeinsame Fallrekonstruktion: & Dokumentation/Visualisierung \\
\hline $\begin{array}{l}\text { - Wie sieht das „Wirkungsgefüge“ im Fall konkret } \\
\text { aus? (Wie wirken die Leistungen der einzelnen Ak- } \\
\text { teure aufeinander? Wo behindern sich Akteure in } \\
\text { der Leistungserbringung? Wie werden Leistungen } \\
\text { aktuell miteinander kombiniert? Wie sind Übergän- } \\
\text { ge/Übergaben gestaltet?) }\end{array}$ & $\rightarrow$ Themenspeicher \\
\hline $\begin{array}{l}\text { - Verändern sich durch die Einbeziehung der unter- } \\
\text { schiedlichen Perspektiven der Nutzenden die mög- } \\
\text { lichen Stellschrauben? }\end{array}$ & \\
\hline $\begin{array}{l}\text { - Sind die Interventionen dadurch neu bzw. anders } \\
\text { zu denken? }\end{array}$ & \\
\hline - Wie sieht die aktuelle Situation aus? & \\
\hline $\begin{array}{l}\text { - Ist eine Falldynamik zu erkennen? Wenn ja, wie } \\
\text { kann sie beschrieben werden? Von welchen Fakto- } \\
\text { ren hängt die Dynamik ab? Wenn nein, wodurch } \\
\text { wird die statische Situation aufrechterhalten? }\end{array}$ & \\
\hline $\begin{array}{l}\text { - Was sind in diesem Fall die „zentralen fallübergrei- } \\
\text { fenden Themen? }\end{array}$ & \\
\hline \multicolumn{2}{|c|}{$\begin{array}{l}\text { Die Phase I sollte mehrere Male durchlaufen werden, es sollten mindestens vier Fälle rekon- } \\
\text { struiert werden. }\end{array}$} \\
\hline \multicolumn{2}{|l|}{ Phase II } \\
\hline $\begin{array}{l}\text { Definition von „fallübergreifenden Themen“ für die } \\
\text { Zielgruppe }\end{array}$ & Dokumentation/Visualisierung \\
\hline $\begin{array}{l}\text { - Was sind in den rekonstruierten Fällen die „fall- } \\
\text { übergreifenden Themen“? }\end{array}$ & \\
\hline $\begin{array}{l}\text { - Welche Akteure sind mit diesem Thema befasst? } \\
\text { Sind sie bereits am Netzwerk beteiligt oder sollten } \\
\text { sie zukünftig einbezogen werden? }\end{array}$ & \\
\hline $\begin{array}{l}\text { Ergänzung/Kontrastierung mit Daten von Sozial-/ } \\
\text { Jugendhilfeplanung }\end{array}$ & Dokumentation/Visualisierung \\
\hline $\begin{array}{l}\text { - Welche Themen zeigen sich auch in der Sozialbe- } \\
\text { richterstattung/Sozialplanung? }\end{array}$ & \\
\hline $\begin{array}{l}\text { - Welche Daten/Erkenntnisse kann die Sozial-/ } \\
\text { Jugendhilfeplanung beitragen, die sich nicht in den } \\
\text { zentralen fallübergreifenden Themen finden? }\end{array}$ & \\
\hline $\begin{array}{l}\text { - Gibt es Widersprüche und wie lassen sie sich erklä- } \\
\text { ren? }\end{array}$ & \\
\hline $\begin{array}{l}\text { Definition von "generativen Themen“ für die Ziel- } \\
\text { gruppe }\end{array}$ & Dokumentation/Visualisierung \\
\hline $\begin{array}{l}\text { Welche „generativen Themen“ ergeben sich aus den } \\
\text { „fallübergreifenden Themen“ und der Ergänzung/ } \\
\text { Kontrastierung durch Daten der Sozial-/Jugendhil- } \\
\text { fe-planung? }\end{array}$ & \\
\hline
\end{tabular}




\begin{tabular}{|c|c|}
\hline TOPs & Wichtig! \\
\hline $\begin{array}{l}\text { - Welche Akteure sind mit diesem Thema befasst? } \\
\text { Sind sie bereits am Netzwerk beteiligt oder sollten } \\
\text { sie zukünftig einbezogen werden? }\end{array}$ & \\
\hline \multicolumn{2}{|l|}{ Phase III } \\
\hline $\begin{array}{l}\text { Entwicklung eines Zielsystems } \\
\text { - Was möchten wir für die Zielgruppe und die Zu- } \\
\text { sammenarbeit grundsätzlich erreichen? Welche } \\
\text { Teilziele und dazugehörigen Ergebnisziele müssen } \\
\text { die beteiligten Organisationen erreichen, um den } \\
\text { erstrebenswerten Zustand für die Zielgruppe herzu- } \\
\text { stellen? Welche Aktivitäten sind dafür nötig und } \\
\text { wer ist für welche Aktivität zuständig? } \\
\text { - Woran erkennen die Akteure die Erreichung der } \\
\text { Ziele, wie können sie den Grad der Zielerreichung } \\
\text { messen? Welche Bedingungen könnten die Zieler- } \\
\text { reichung beeinflussen? } \\
\text { Definition des Grundsatzziels, Definition der Rah- } \\
\text { menziele und dazugehörigen Ergebnisziele } \\
\rightarrow \text { Definition von Aktivitäten und der Zuständig- } \\
\text { keiten der } \\
\text { Akteure für diese Aktivitäten sowie Definition von } \\
\text { Indikatoren, Quellen und Annahmen }\end{array}$ & Dokumentation/Visualisierung \\
\hline
\end{tabular}

\subsubsection{Umsetzung des Zielsystems}

Das Netzwerk legt im nächsten Schritt eine gemeinsame Arbeitsplanung auf der Basis der für jedes Rahmenziel definierten Ergebnisziele fest. Die Arbeitsplanung beinhaltet Aktivitäten auf verschiedensten Ebenen. Die Umsetzung des Zielsystems ermöglicht und erfordert veränderte Prozesse und Abläufe und Verfahren, die hier ebenfalls methodisch beschrieben werden. Welche Produkte im Einzelnen geplant werden, hängt von den spezifischen lokalen Gegebenheiten ab. Die hier beschriebenen stehen exemplarisch für weitere. Die Umsetzung nicht-komplexitätsreduzierter Fallrekonstruktionen in ein Zielsystem hat zur Folge, dass das Zielsystem thematisch eine große Bandbreite umspannt und ein ganzer Katalog an Aktivitäten entstehen kann. Die Pragmatik von Umsetzungen erfordert aber eine Priorisierung der Aktivitäten. Es obliegt daher den Netzwerkkoordinierenden mit den Methoden des Projektmanagements der Vielzahl möglicher und notwendiger Aktivitäten eine Struktur zu geben, so dass die nächsten Schritte des Netzwerks geplant werden. Optimal ist ein Mix aus kurzfristig und mittelfristig umsetzbaren und erreichbaren Aktivitäten, die 
einen unmittelbaren Nutzen für die Zielgruppe haben und mit denen das Netzwerk auch in der Öffentlichkeit auf sich aufmerksam machen kann. Die Priorisierung muss berücksichtigen, dass alle Akteure des Netzwerks eingebunden sind und eine aktive Rolle haben. Erste Lerneffekte können eine Vorbildfunktion haben und Motivation stiften.

\subsubsection{Prozesse}

Zentrales Ziel der Produktionsnetzwerke ist es, die Versäulung in der Versorgungsstruktur aufzubrechen und funktionale getrennte, aber prozessual zusammengehörige Leistungen als durchgängig organisierte Versorgungsstruktur der Zielgruppe zur Verfügung zu stellen. Dazu sind die Entwicklung von Dienstleistungsketten, die Vereinbarung von Standards und Verfahren sowie Schnittstellenregelungen notwendig.

\subsection{Dienstleistungsketten ${ }^{31}$}

Der Ausgangspunkt sind die integrierten Fallrekonstruktionen und die "generativen Themen“, auf deren Basis die Rahmenziele und Ergebnisziele bereits arbeits- und politikfeldübergreifend formuliert werden. Zur Erreichung dieser und zur konkreten Ausgestaltung der Dienstleistungsketten entwickelt das Netzwerk passende Aktivitäten, die zusammen mit weiteren Einzelleistungen der am Netzwerk beteiligten Akteure zu Dienstleistungsketten verknüpft werden. Dabei liegt das Augenmerk gerade auch auf den Schnittstellen und Übergängen zwischen den Organisationen, um Brüche sowie Parallelleistungen zu vermeiden. Dazu gehören auch Koordinationsabsprachen sowie die Festlegung von Bearbeitungs- und Verfahrensstandards und Verantwortlichkeiten.

Anstelle von Einzelleistungen oder feldinternen Bausteinen wird die gesamte Komplexität des Ablaufs in den Blick genommen. Das Netzwerk prüft bestehende Strukturen aus der Perspektive der Nutzenden und schafft somit ggf. neue Strukturen, die evident sind, auch ohne dass akut ein Fall zu lösen ist. Dienstleistungsketten verbinden einen gewünschten Endzustand mit einem faktischen Anfangszustand und sind damit grundsätzlich sequenziell, was aber nicht heißt, dass alle Einzelleistungen sequenziell aufeinander folgen müssen. Vielmehr sind parallele Leistungen

31 Vgl. ausführlich Abschnitt 4.7. 
oft sinnvoll. Für die Konzipierung und konkrete Erarbeitung von Dienstleistungsketten wird am Zielsystem angesetzt (vgl. Abschnitt 4.6.2.3); dabei sind folgende Fragen zu klären:

- Was soll prioritär vorgenommen werden?

- Welche Aktivitäten sind nützlich?

- Welche neuen Aktivitäten müssen mit Blick auf die Erreichung der Ergebnisziele entwickelt werden, welche vorhandenen angepasst werden?

- Wer ist rechtlich zuständig?

- Wer muss beteiligt sein?

- Welche Aktivitäten gehören zusammen?

- Wie kann ein gemeinsamer Planungsprozess gestaltet werden?

- Was ist ein sinnvoller Ablauf innerhalb einer Dienstleistungskette?

- Was muss koordiniert werden?

- Welche Übergabepunkte zwischen den Leistungsprozessen einzelner Akteure gibt es?

- Wie sind die Übergänge an diesen Schnittstellen möglichst „nutzerfreundlich" und effektiv zu gestalten?

- Welche fachlichen Standards und/oder Verfahrensweisen müssen definiert und abgestimmt werden?

- Für welche Leistungsbestandteile braucht es Beteiligte aus dem Netzwerk, für welche können „Externe“ beauftragt werden?

- Wer hat bei was die Federführung? Welche Aufgaben umfasst die „Federführung"?

Dienstleistungsketten können (Teil-)Leistungen umfassen, die von Netzwerkpartnern erbracht werden, und solche, für die Auftragsverhältnisse ausreichen. Es ist deswegen jeweils zu klären, welche Grade professioneller und/oder organisationaler Autonomie existieren und auch beibehalten werden müssen, um eine optimale gemeinsame Leistung zu ermöglichen. Entsprechend sind sowohl die Beziehungen innerhalb des Netzwerks zu gestalten als auch die Beziehungen zwischen Netzwerkpartnern und anderen nicht am Netzwerk beteiligten Anbietern von Dienstleistungen. Hilfreich hierfür kann eine erneute Beteiligtenanalyse (vgl. Abschnitt 4.6.1.2) sein.

Die Konzipierung von Dienstleistungsketten ermöglicht einen hohen Grad an Flexibilität, was gerade bei komplexen Fallkonstellationen hilfreich sein kann.

Die Umsetzung erfolgt in folgenden Schritten:

- Einladung der (wichtigsten) Akteure, die zur Umsetzung einer Dienstleistungskette notwendig sind, in Arbeitsgruppen. 
- Klärung der oben formulierten Fragen - vor diesem Hintergrund werden dann zielgruppenbezogene Dienstleistungsketten definiert.

- Definition von Leistungsprozessen in der Form von Flussdiagrammen für die einzelnen Dienstleistungsketten.

- Gemeinsame Diskussion, Überprüfung und Festlegung der für die Messung der Zielerreichung sinnvollen Indikatoren.

- Planung der für die Umsetzung der Dienstleistungsketten benötigten Ressourcen (inklusive der benötigten Kompetenzen).

Zur praktischen Umsetzungsplanung von einzelnen Leistungsprozessen können Ablaufpläne erstellt werden, um Abläufe und Übergabepunkte zu definieren. Mit den Methoden und Symbolen der Flowcharts werden Leistungsprozesse visualisiert und neu modelliert, so dass eine für alle Beteiligten verbindliche Grundlage entsteht.

\subsection{Standards und Verfahren}

Die Fallrekonstruktionen haben auf vielfältige Weise gezeigt, dass Standards und Verfahren der Fallbearbeitung in den einzelnen Organisationen einen maßgeblichen Einfluss auf die Unterstützung der Zielgruppe haben. Vor dem Hintergrund einer komplexen Fallsicht erweisen sie sich in einigen Bereichen als nicht zielführend. Vergleicht man die Standards der einzelnen Organisationen untereinander wird deutlich, dass diese teilweise sehr unterschiedlich bis widersprüchlich sind. Auch zeigt sich, dass einzelne Verfahren wie bspw. eine umfassende Falleinschätzung bei mehreren Organisationen unabhängig voneinander vorgenommen werden.

Ein wesentlicher Arbeitsschritt im Netzwerk stellt die kritische Überprüfung dar, in wie weit die organisationalen Verfahren angepasst werden können, um eine komplexe Fallbearbeitung zu unterstützen. Ebenso müssen Verfahren und Standards der am Netzwerk beteiligten Organisationen untereinander abgestimmt werden. Im Rahmen von Arbeitsgruppen können diese vergleichend geprüft und daraufhin gemeinsame Leitlinien entworfen werden. Das Ziel ist die Standards und Verfahren in ihrer Ausrichtung fachlich zu synchronisieren und Widersprüche und Redundanzen zu minimieren. Ein Praxisbeispiel ist die gemeinsame Erarbeitung von Indikatoren der Zugangssteuerung und einer abgestimmten Fallanamnese. 
4.6.3.1.3 Schnittstellen-, Kommunikations- und Abstimmungsregelungen

Schnittstellenregelungen sind immer dann notwendig, wenn ein Bedarf oder Leistungen für die Zielgruppe von mehr als einer Organisation erbracht wird. Die Fallrekonstruktionen zeigen, an welchen dieser „Nahtstellen" es häufiger dazu kommt, dass Übergaben nicht stattfinden, die Zugangssteuerung nicht geklärt ist, Kompetenz- oder Zuständigkeitsstreitigkeiten entstehen oder andere Fall- und Problemsichten dazu führen, dass Prozesse nicht (mehr) fortgeführt werden, sondern versanden.

Schnittstellenregelungen beinhalten neben Lösungen zu eben diesen Problemkonstellationen auch die zentrale Frage der gemeinsamen Verantwortung für den Gesamtprozess. Kommunikations- und Abstimmungsregelungen erleichtern bspw. festzulegen, dass abgebende Organisationen in der Bringschuld für eine geordnete und nachvollziehbare Übergabe an die aufnehmende Organisation sind. Umgekehrt ist zu regeln, dass und wie die aufnehmende Organisation an der geleisteten Vorarbeit ansetzt und die Arbeit des Prozessvorgängers nutzt. Deshalb ist es wichtig, dass bei Schnittstellen, die nicht zur Gänze vordefiniert sind, beide Seiten für die Übergabe verantwortlich sind und sich auch gemeinsam in dieser Verantwortung sehen.

\subsubsection{Produkte}

\subsection{Neue und gemeinsame Angebote}

Die Fallrekonstruktionen geben Hinweise auf die Qualität der Unterstützungsstruktur für die Zielgruppe und im Sozialraum. Angebotslücken oder Doppelstrukturen können erkannt werden. Die Fragen von Bedarfsorientierung und Passförmigkeit können aus den unterschiedlichen disziplinären Perspektiven im Netzwerk diskutiert werden. Die Zugänge der Zielgruppe zu den Angeboten und die Fragen von angemessener Beteiligung und Nutzung der Angebote werden deutlich. Dies leitet eine kritische gemeinsame Prüfung der Angebote und eine stärker abgestimmte Angebotsplanung ein. Die im Netzwerk vertretenen Fachlichkeiten ermöglichen eine umfassendere Planung und die gemeinsame Durchführung von Angeboten, die wiederum nach Außen das Zusammenwirken des Netzwerks dokumentieren. 


\subsection{Fallkonferenzen}

In Fallkonferenzen treten Akteure der am Netzwerk beteiligten Organisationen nach einem geregelten Verfahren zusammen, um über einzelne konkrete „Fälle“ zu sprechen und gemeinsame Lösungen zu finden und Verfahren zu erproben, die in der Planungskonferenz vereinbart werden. Im Unterschied zur Planungskonferenz (vgl. Abschnitt 4.6.2) geht es in Fallkonferenzen explizit darum, die besprochenen und gemeinsam rekonstruierten Fälle auch „zu lösen“, d. h. konkrete auf den Einzelfall bezogene Strategien und ggf. Interventionen zu entwickeln.

Fallkonferenzen sollten ein regelmäßiges Arbeitsinstrument sein und in fest verabredeten Abständen stattfinden. Fallkonferenzen stärken darüber hinaus das Verständnis der Netzwerkpartner für unterschiedliche Perspektiven und Arbeitsweisen der Partner und damit die Zusammenarbeit der Organisationen.

Für eine Fallkonferenz eignen sich Fälle, die besonders komplex sind und nicht bilateral zwischen zwei Akteuren geklärt werden können. Für die Durchführung von Fallkonferenzen muss auf der Ebene des Netzwerks (d. h. in der „Planungskonferenz") ein Verfahren vereinbart werden. Dazu gehört die Form, in der die Fälle eingebracht werden. Die Fälle können (durch die einbringende Organisation) vorab kurz beschrieben und an die anderen Netzwerkpartner zur Kenntnis gegeben werden, um ihnen die Möglichkeit der Vorbereitung zu geben. Alternativ kann der Fall auch ohne vorab verschicktes Material besprochen werden. Die Fallbeschreibung sollte die Problemkonstellation, den bisherigen Fallverlauf, bereits erfolgte Hilfen und Maßnahmen und eine in der Fallkonferenz zu behandelnde Frage beinhalten. Je nach Vereinbarung ist ein vorab bestimmter Akteur (unter Umständen auch im Rotationsverfahren) oder auch die Netzwerkkoordination für die Vorbereitung einer Fallkonferenz zuständig. Zur Vorbereitung gehören die Einladung, wenn vereinbart, die Verteilung der Fallbeschreibungen und ggf. die Auswahl der zu beteiligenden Akteure.

Datenschutzrechtliche Bestimmungen müssen unbedingt beachtet werden. Eine Fallkonferenz darf nur mit Einverständnis und entsprechender Schweigepflichtsentbindung der Nutzenden stattfinden. In einer Fallkonferenz sollten alle Netzwerkpartner vertreten sein. Es ist auch möglich, einen festen Kern von immer zu beteiligenden Akteuren (z. B. Jobcenter, Jugendamt) festzulegen und andere je nach Lage des Falles einzuladen. Im Einzelfall kann es auch Sinn machen, Akteure einzuladen, die (bisher) keine Netzwerkpartner sind. Mögliche Lösungen müssen rasch in deren Hierarchie abgesichert werden können. Die Moderation einer Fallkonferenz 
muss im Vorfeld geklärt werden. Rotierende Verfahren sind auch hier gut geeignet. Die Person, die einen Fall einbringt, sollte nicht moderieren. In Fallkonferenzen können die beteiligten Netzwerkpartner Situationen und Handlungsstrategien anhand konkreter Einzelfälle diskutieren und so gemeinsame Vorgehensweisen vereinbaren. Eine gute Dokumentation der Ergebnisse ist aus zwei Gründen wichtig: Zum einen können vereinbarte Lösungswege für den jeweiligen Fall schriftlich fixiert werden; zum anderen kann die Dokumentation der Fallkonferenzen illustrieren, wie sich die Zusammenarbeit der Netzwerkakteure verändert hat, wo noch Regelungsbedarfe bestehen und dies als Rückkopplung in die „Planungskonferenz“ geben. Auch hier ist die Einhaltung der datenschutzrechtlichen Bestimmungen selbstverständlich. Bei der Konzipierung von Fallkonferenzen ist auch zu prüfen, ob und welche Fallkonferenzen es im Feld bereits gibt und wie diese ggf. für das Netzwerk zu nutzen wären.

\subsection{Angebotsüberblick}

Ein Angebotsüberblick (Wegweiser, Broschüre, Branchenbuch oder ähnliches) bietet der Zielgruppe und/oder den Netzwerkakteuren einen systematischen Überblick über die Leistungen und Unterstützungsangebote vor Ort. So wird die Suche nach der richtigen Unterstützung zielgerichteter und damit schneller. Der Zielgruppe wird der Weg durch die Angebotsvielfalt geebnet. Für die operativen Fachkräfte ermöglicht die Angebotsübersicht eine Qualifizierung ihrer Beratungspraxis. Der Überblick kann auch zu einem „Wegweiser“ weiterentwickelt werden, der auch Anknüpfungspunkte zwischen den Angeboten aufzeigt.

Für die Konzeption ist nicht nur entscheidend, ob sich der Überblick an die Benutzer oder die Fachkräfte - oder aber an beide - richtet, sondern auch, ob ausschließlich auf die Angebote des Netzwerks, des Sozialraums oder der gesamten Stadt/ des Landkreises verwiesen wird. Auch kann eine Broschüre ausschließlich internetgestützt oder als gedruckte Version entwickelt werden. Gleichzeitig ermöglicht die Arbeit an einer Broschüre den Einstieg in die gemeinsame Beurteilung der Angebote vor Ort und geht so über eine reine Informationssammlung hinaus und wird $\mathrm{zu}$ einem wichtigen Arbeitsinstrument an der Unterstützungsstruktur. Die Broschüre macht das Netzwerk zudem bei der Zielgruppe und in der Öffentlichkeit sichtbar. Sie kann sinnvoll in der Öffentlichkeitsarbeit des Netzwerkes präsentiert werden. 


\subsection{Projekte}

Die Erprobung von neuen kooperativen Strukturen kann auch im Rahmen eines Projekts stattfinden. Dessen lokale und zeitliche Begrenzung ermöglicht es, Erfahrungen zu machen, die die Basis für eine kontinuierliche Zusammenarbeit, z. B. die Entwicklung von Dienstleistungsketten, legen. Projekte bieten sich gerade auch bei Handlungsfeldern an, die nur einen geringen Formalisierungsgrad aufweisen sowie bei in sich sehr heterogenen Handlungsfeldern, die nicht durch einen bzw. wenige Akteure repräsentiert werden können. In einem Projekt können Ansätze exemplarisch erprobt werden, die Strahlkraft für das gesamte Feld entwickeln.

\subsection{Systematischer Informationsaustausch}

Für kooperative Arbeitsstrukturen und abgestimmte Dienstleistungen und Prozesse ist es notwendig, dass vor allem die mittlere Führungsebene und die operativen Fachkräfte einen aktuellen und fundierten Wissensstand über die angrenzenden Arbeitsgebiete, Rechtskreise und Organisationen haben. Dies kann durch einen regelmäßigen und verbindlichen Informationsaustausch auf der operativen Ebene, durch gemeinsame Weiterbildungsveranstaltungen oder wechselseitige Hospitationen sowie auch durch die Fallkonferenzen erreicht werden. Entscheidend ist, dass die Organisationen Gelegenheiten schaffen zur verbindlichen Reflexion der gemachten Erfahrungen und der Informationen für die Bedeutung der je eigenen Arbeitsansätze und -abläufe.

\subsection{Workshops mit der operativen Ebene und Zwischenworkshops}

Vor der Umsetzung der Ergebnisse aus den Planungskonferenzen auf der operativen Ebene bietet sich vor allem für die initiierende Organisation ein Zwischenworkshop mit den Fachkräften an, die im operativen Bereich für den Sozialraum zuständig sind. Der Zwischenworkshop ermöglicht, dass das Zielsystem bekannt gemacht wird und die Ergebnisziele und die Aktivitäten ergänzt und durch Praxiserfahrungen überprüft werden. Dabei können weitere Hinweise zu Verfahrensweisen und Praxisproblemen gesammelt werden. Das Projekt wird nachvollziehbar und die Dimensionen der anstehenden Veränderungen werden in der Organisation verortet und 
legitimiert. Bei sozialräumlich ausgerichteten Netzwerken kann so auch eine Übertragung auf andere Sozialräume vorbereitet werden.

Neben dem Zwischenworkshop bieten sich insgesamt Workshops mit den operativen Ebenen an. Gerade in größeren Organisationen können so die Arbeitsergebnisse des Netzwerks zu signifikanten Zeitpunkten kommuniziert werden und erfahrene Fachkräfte können mit ihren Ideen und Hinweisen die Qualität der Ergebnisse sichern, bevor diese implementiert werden.

\subsection{Kooperationsvereinbarungen}

Strukturen der Zusammenarbeit zwischen den Organisationen können durch förmliche Kooperationsvereinbarungen festgelegt werden.

Die in der Praxis erprobten Erfahrungen zeigen, dass Kooperationsvereinbarungen im gesamten Netzwerk oder als bilaterale Absprachen bzw. Vereinbarungen fixiert werden können. Mit Kooperationsvereinbarungen können die entwickelten Prozesse gesichert werden. Sie können aber auch am Anfang der Umsetzung stehen, indem hierüber ein verbindlicher Rahmen für die Erarbeitung und Erprobung von konkreten gemeinsamen Aktivitäten geschaffen wird. Wesentlich ist, dass die Kooperationsvereinbarung neben den Zielen der Vereinbarung und der grundsätzlichen Haltung der Zusammenarbeit konkrete Regelungen zum Informationsaustausch zwischen den Partnern, zur Zusammenarbeit im Einzelfall, zur fallübergreifenden Zusammenarbeit sowie zur Evaluation auf der operativen Ebene enthält.

\subsubsection{Verstetigung und Überprüfung}

Produktionsnetzwerke sind verbindlicher als andere Netzwerktypen, z. B. als Informationsnetzwerke. Damit stellt sich die Frage, wie Netzwerkstrukturen geschaffen und stabilisiert werden können.

Um die Arbeitsfähigkeit zu behalten, ist es unter Umständen sinnvoll, Substrukturen zu schaffen, z. B. Arbeitsgruppen für einzelne Teilziele. Dabei ist die Dynamik der Netzwerkentwicklung zu beachten: Im Laufe der Zeit kann sich ein Netzwerk durch thematische Ausdifferenzierung verkleinern oder aber durch die Beschäftigung mit zusätzlichen Inhalten und die Einbeziehung neuer Partner vergrößern. Ein wichtiges Element der Stabilisierung ist die beständige Bekräftigung von „Verlässlichkeit“, die 
sich im gegenseitigen „Geben und Nehmen“ realisiert. Verlässlichkeit zeigt sich z. B. in der Verbindlichkeit im Hinblick auf die Einhaltung von Absprachen und Netzwerkregeln. Diese Verbindlichkeit korrespondiert mit dem inneren Zusammenhalt des Netzwerks. Diesen festigen die Netzwerkakteure, wenn sie folgendes beachten:

- Die Akteure haben gemeinsame Ziele definiert. Deren Gültigkeit muss von Zeit zu Zeit überprüft werden.

- Die Realisierung der vereinbarten Aktivitäten und Projekte muss begleitet werden. Dazu gehören die Vereinbarung von Zeitplänen und die gegenseitige Information über das Fortschreiten oder Störungen bei der Umsetzung, aber auch die Überprüfung von Zuständigkeiten.

- Die Zusammenarbeit wird verbindlicher, wenn gemeinsam Regeln entwickelt und schriftlich niedergelegt werden. Generell sollten wesentliche Arbeitsergebnisse dokumentiert werden.

- Die Dienstleistungsketten müssen im Rahmen von Planungskonferenzen überprüft und bewertet werden, eine stabile Struktur im Netzwerk ist die Voraussetzung dafür, Brüche und Lücken bei der Unterstützung der Zielgruppen erkennen, offen ansprechen und bearbeiten zu können. Dazu gehört es auch, eventuell weitere Akteure zu identifizieren und für die Netzwerkarbeit zu gewinnen.

- Nicht zuletzt müssen die Ressourcen für die Netzwerkarbeit realistisch benannt sein und Zeitpläne und Umsetzungsprojekte darauf abgestimmt sein.

\subsubsection{Regeln und Vereinbarungen}

Gemeinsam erarbeitete, verbindliche und schriftlich festgehaltene Netzwerkregeln gestalten die Beziehungen innerhalb des Netzwerks. Netzwerke sind freiwillige Zusammenschlüsse von unabhängigen Organisationen. Deshalb brauchen sie eigene Regeln. Denn Arbeitsweisen, Aufträge und Regeln der Akteure aus den verschiedenen beteiligten Organisationen können sehr unterschiedlich sein.

Zu den Themen der Netzwerkregeln gehören die Teilhabe am Netzwerk (Eintritt, Austritt), der organisatorische Aufbau und die Abläufe im Netzwerk sowie die interne und externe Kommunikation. An Netzwerkregeln stellen sich besondere Anforderungen:

- Sie sind so zu gestalten, dass die gemeinsame Arbeit im Netzwerk bestmöglich unterstützt wird. 
- Sie müssen gemeinsam mit allen Akteuren erarbeitet und verabschiedet werden.

- Sie müssen mit den Aufträgen und Zielen der beteiligten Organisationen vereinbar sein.

- Sie müssen klar und verbindlich sein, aber flexibel gegenüber neuen Anforderungen und Impulsen.

- Sie sollten immer schriftlich fixiert werden.

- Alle Beteiligten sind gleichermaßen für die Einhaltung der Regeln verantwortlich.

- Die Netzwerkkoordination achtet auf die Einhaltung und eventuellen Änderungsbedarf.

Abstimmungsregeln dienen dazu, die Abläufe im Netzwerk so zu gestalten, dass sie den organisationalen Aufbau des Netzwerkes optimal unterstützen. Es gibt unterschiedliche Ebenen der Abstimmungsregeln im Netzwerk. Regeln für Entscheidungen klären folgende Fragen:

- Wie werden Veränderungsbedarfe aus der Planungskonferenz in die Organisationen getragen?

- Wie werden organisationsinterne Entscheidungen in das Netzwerk getragen?

- Wie kann das Netzwerk Entscheidungen von einzelnen Akteuren herbeiführen oder verlangen?

- Welche Sachverhalte sind in welcher Weise zu entscheiden?

Regeln für Arbeitsgruppen, Teilnetzwerke und Projekte betreffen das Verhältnis zwischen Gesamtnetzwerk und Substrukturen. Die Planungskonferenz sollte so zusammengesetzt sein, dass operative und Leitungsebene dauerhaft repräsentiert sind. Im Einzelnen werden darüber hinaus trotzdem Unterarbeitsgruppen und Teilnetzwerke notwendig sein (insbesondere bei sozialräumlich ausgerichteten Netzwerken). In diesen müssen folgende Fragen geklärt werden:

- Wie werden Aufträge an Arbeitsgruppen oder Teilnetzwerke vergeben (Arbeitsauftrag)?

- Sind Aufträge klar und transparent formuliert?

- Sind eventuelle Zielkonflikte geklärt, bevor Aufträge an Untergruppen vergeben werden?

- Sind die Zuständigkeiten für Arbeitsaufträge, Projekte und kontinuierliche Aufgaben von allen anerkannt?

- Wie werden Fristen für Arbeitsergebnisse terminiert? Wie werden Prioritäten festgelegt?

- Wie werden Arbeitsaufträge nachverfolgt? 
- Wie bringen Arbeitsgruppen ihre Ergebnisse in Entscheidungsprozesse ein oder führen in ihren Vorhaben entsprechende Entscheidungen herbei?

Regeln für die Zielüberprüfung und den Informationsaustausch betreffen die Binnenstruktur des Netzwerks:

- Wann und wie oft findet eine gemeinsame Überprüfung der vereinbarten Ziele und deren Erreichungsgrad statt?

- Wie wird aus Arbeitsgruppen, Teilnetzwerken oder Kernnetzwerken berichtet? Wer sind die Adressatinnen und Adressaten der Berichte? Wie ist der Informationsfluss geregelt? Wie wird Transparenz tatsächlich hergestellt? Müssen Informationen abgefordert werden oder erfolgen sie nach einem im Voraus abgestimmten Verfahren?

Abstimmungsregeln mit der Umwelt des Netzwerks beziehen sich auf die Darstellung nach außen des Netzwerks:

- Wie wird entschieden, wann Ergebnisse der Netzwerkarbeit veröffentlicht werden können?

- Wer übernimmt die Öffentlichkeitsarbeit: die Netzwerkkoordination, die Planungskonferenz, die Leitungsebene einzelner Akteure oder aller Akteure?

\subsubsection{Monitoring und Dokumentation}

Das ständige und begleitende Monitoring der Ziele und Aktivitäten sowie des Zielerreichungsgrades und ggf. deren Anpassung und Neuausrichtung ist notwendig für den Erfolg des Netzwerks.

Mit der Erarbeitung eines Zielsystems wird bereits die Grundlage für diese Art von Evaluation geschaffen, denn schon zu diesem Zeitpunkt werden neben den Zielen weitere Größen definiert, die dann für das Monitoring hilfreich sind:

- Indikatoren, die den Messpunkt benennen, an dem die Zielerreichung festgestellt wird. Sie sollten Aussagen zur Qualität, zur Quantität und zur Zeitvorstellung der Zielerreichung beinhalten. Indikatoren dienen also dazu, den Erreichungsgrad zu überprüfen.

- Quellen, die die Materialbasis für die Überprüfung darstellen und die Daten bezeichnen, an denen die Messung vorgenommen wird. Sie werden den einzelnen Indikatoren entsprechend zugeordnet.

- Annahmen sind dann zu benennen, wenn die geplanten Ergebnisse erst durch zusätzliche, nicht durch die Akteure steuerbare, Ereignisse negativ oder positiv beeinflusst werden können. 
Weil die Erkenntnisse der Planungskonferenzen unter Beteiligung der Sozialplanung gewonnen wurden und die Daten der Sozialplanung in die Definition von Quellen und Indikatoren eingeflossen sind, können Daten der Sozialplanung selbstverständlich auch bei der Bestimmung der Zielerreichung eingesetzt werden. Kritisch dagegen ist es $\mathrm{zu}$ sehen, wenn der „Erfolg“ des Netzwerks ausschließlich an Zahlen des Controllings (z. B. Daten eines Integrationsmonitorings) und nicht an der Erreichung der im Netzwerk vereinbarten Ziele gemessen wird. Daten, die aus einer organisationsübergreifenden Dokumentation im Rahmen des Netzwerks (z. B. durch gemeinsame EDV-gestützte Dokumentationssysteme) gewonnen werden, können für die Sozialplanung genutzt werden. Ein regelhaftes Monitoring ermöglicht einen Umgang mit verschiedenen Problemstellungen in der Umsetzung:

- Eine de facto von der Planung abweichende Zielpriorisierung, d. h. bestimmte Ergebnisziele werden vernachlässigt bzw. aus den Augen verloren.

- Aktivitäten sind von unterschiedlichen Akteuren zu erbringen, um ein Ziel zu erreichen. Ziehen hierbei nicht alle an einem Strang oder in unterschiedliche Richtungen, kann das Ziel nicht verwirklicht werden.

Diese und ähnliche Schwierigkeiten müssen von der Netzwerkkoordination beobachtet werden, um sie mit den beteiligten Akteuren zu kommunizieren. Rückschlüsse für den weiteren Projektverlauf - und damit einhergehend für das Zielsystem - können dann gemeinsam entschieden werden. Eine Möglichkeit, die Ziele während des Prozesses zu überprüfen und ggf. anzupassen, sind weitere Planungskonferenzen.

Voraussetzung für Verbindlichkeit in der Zusammenarbeit und Grundlage für das Monitoring ist eine angemessene Dokumentation. Durch schriftliche Aufzeichnungen werden die Vorhaben, die einzelnen Schritte und Aktivitäten, die Vorgehensweise sowie die Entscheidungen und Ergebnisse des Netzwerks festgehalten und nachvollziehbar gemacht. Je nach Anlass und adressierter Person bieten sich methodisch unterschiedliche Formen an, die von kurzen internen Protokollen bis zu umfangreichen Berichten reichen. Mit der Dokumentation werden drei Ziele erreicht:

- Schriftliche Dokumente ermöglichen einen Überblick über das Netzwerk und seine Arbeit.

- Die Kommunikation zwischen den Akteuren ist sichergestellt und funktioniert auf diese Weise auch unabhängig von Personen. Dies ist besonders wichtig bei ausdifferenzierten Netzwerken mit verschiedenen Arbeitsgruppen. 
- Anhand der Dokumentation lässt sich der Fortgang des Netzwerks beobachten; sie erleichtert einen regelmäßigen Rückblick („Tun wir das Richtige auf die richtige Weise?").

Die Einführung einer systematischen Dokumentation kann folgendermaBen erfolgen:

- Im ersten Schritt werden Art, Umfang, Detaillierungsgrad und Häufigkeit festgelegt.

- Je nach Ressourcen wird eine Person - ggf. die Netzwerkkoordination - dauerhaft für die Erstellung der Dokumente benannt; alternativ kann die Verantwortung rotieren. Wichtig ist es, den Verteiler für die Unterlagen und den Umgang mit noch intern zu behandelnden Dokumenten zu verabreden.

- Allen Akteuren muss klar sein, dass sie einen aktiven Part haben, indem sie Dokumentationspflichten nachkommen und Aktivitäten aufmerksam verfolgen.

Folgende Instrumente bieten sich an:

- Protokolle unterscheiden sich nach ihrem Charakter: Es ist festzulegen, ob Verlaufs- oder Ergebnisprotokolle angefertigt werden, ob die Protokolle (Ergebnisprotokoll) reinen Dokumentationscharakter haben oder auch Arbeitsaufträge beinhalten.

- Statusberichte dokumentieren festgelegte Vorhaben, Umsetzungsschritte, Ergebnisse und weiteren Handlungsbedarf.

- Zwischen- oder Abschlussberichte bieten sich an, wenn umfangreichere Arbeitspakete einen Zwischenstand erreicht oder Arbeitsgruppen ihren Auftrag beendet haben.

- Internetbasierte Plattformen dienen der Darstellung von Informationen und Produkten des Netzwerks in der (Fach-)Öffentlichkeit. Geschützte Bereiche im Internet ermöglichen den nicht-öffentlichen Austausch zwischen den Akteuren des Netzwerks.

- Kurzberichte sind eine gute Möglichkeit, die Herkunftsorganisationen der Akteure im Netzwerk, politische Gremien, die lokale Fachöffentlichkeit oder andere örtliche Netzwerke über Ergebnisse aus dem Netzwerk - aktuell - zu informieren.

- Bei der Dokumentation von Fallrekonstruktionen ist auf den Datenschutz zu achten. Entsprechende Regelungen sollten dazu im Netzwerk vereinbart werden. 


\subsubsection{3 Überprüfung und Reflexion der Dienstleistungsketten}

Eine wichtige Arbeitsmethode ist hierbei die Überprüfung der Dienstleistungsketten an ausgewählten Fällen, d. h. die Weiterarbeit an den Fallkonstellationen. Im Zuge dessen werden entlang des fallspezifischen Wirkungsgefüges das Zielsystem und die darauf basierenden Vereinbarungen, geänderten Übergänge und Leistungsprozesse getestet. Relevante Fragestellungen sind hier:

- Entsprechen die entwickelten Dienstleistungsketten/Leistungsprozesse den Fallrekonstruktionen?

- Wo liegen Unstimmigkeiten?

- Sind diese „konstruktionsbedingt“ oder basieren sie auf den Unklarheiten/der Komplexität des dargestellten Falles?

Grundsätzlich bieten sich für die Prüfung zwei Varianten an:

- Die Person, bei der der Fall aufläuft, analysiert die Ausgangskonstellation und greift in der Bearbeitung auf Dienstleistungsketten oder andere Vereinbarungen zurück, ohne dass eine Fallkonferenz notwendig wäre. Diese Variante erfordert ein hohes diagnostisches Potenzial in der Fallbearbeitung.

- Die Person, bei der der Fall aufläuft, beruft eine Fallkonferenz ein (vgl. Abschnitt 4.6.3.2.2). Dort werden eine Fallrekonstruktion, Ziele und Umsetzungsstrategien diskutiert und festgelegt. Für Letztere wird auf die gemeinsamen Vereinbarungen zurückgegriffen. Für die Durchführung von Fallkonferenzen empfiehlt sich eine bestimmte methodische Festlegung.

Die Überprüfung anhand von Verläufen von Fällen gilt auch für andere vereinbarte Projekte des Netzwerkes (z. B. die Beobachtung, ob ein Wegweiser dazu führt, dass Angehörige der Zielgruppe bestimmte Angebote häufiger oder früher wahrnehmen).

Auf alle Fälle sollte die Zusammenarbeit in der Fallarbeit bei Fallabschluss kritisch betrachtet werden, d. h. eine Auswertung der Fallarbeit stattfinden:

- Wo gab es Unstimmigkeiten? Traten sie in Bezug auf die Dienstleistungsketten, die Kooperationsverabredungen, die Prozess- und Netzwerkkooperation auf?

- Welche Veränderungen/Verbesserungen sind erforderlich?

Die Ergebnisse der gemeinsamen Auswertung können dann wiederum an die Planungskonferenz berichtet werden (vgl. Abschnitt 4.6.2). Hier werden Leistungsprozesse auf der strukturellen Ebene immer wieder überprüft und ggf. modifiziert. 


\subsubsection{Zusammenfassung}

Im Folgenden werden die einzelnen Schritte zusammengefasst.

\begin{tabular}{|c|c|c|c|}
\hline $\begin{array}{l}\text { Gliederung } \\
\text { methodisches } \\
\text { Vorgehen }\end{array}$ & Inhalte & Methoden & $\begin{array}{l}\text { Handlungs- } \\
\text { schritte }\end{array}$ \\
\hline 1. Vorentscheidungen & 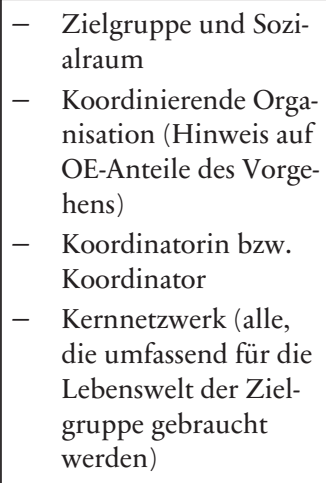 & $\begin{aligned}- & \text { Kartenabfrage } \\
- & \text { Beteiligten- } \\
& \text { analyse }\end{aligned}$ & $\begin{array}{l}\text { 1. Zusammen- } \\
\text { stel-lung des } \\
\text { Netzwerks } \\
\text { auf der Basis } \\
\text { konkreter } \\
\text { Fälle }\end{array}$ \\
\hline 2. Planungskonferenz & & 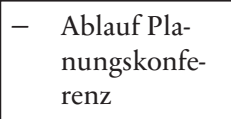 & \\
\hline $\begin{array}{l}\text { 2a. Integrierte Fallre- } \\
\text { konstruktion }\end{array}$ & 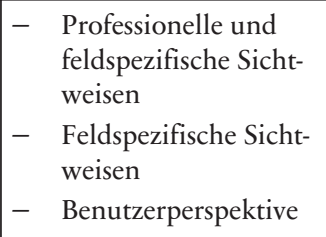 & \begin{tabular}{|ll}
- & Falldarstel- \\
& lung \\
- & Fallverstehen \\
- & Fallrekon- \\
& struktion
\end{tabular} & $\begin{array}{l}\text { 2. Integratives } \\
\text { Fallverste- } \\
\text { hen }\end{array}$ \\
\hline $\begin{array}{l}\text { 2b. Generative The- } \\
\text { men }\end{array}$ & 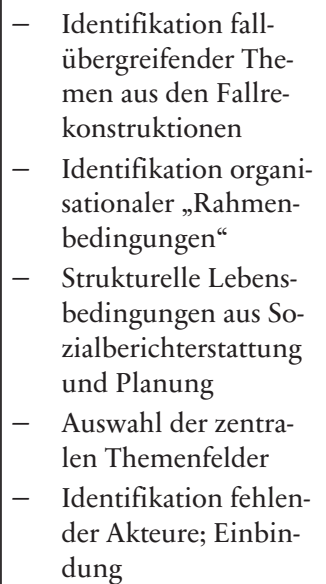 & $\begin{aligned}- & \text { Fallrekon- } \\
& \text { struktion } \\
- & \text { Themenspei- } \\
& \text { cher } \\
- & \text { Beteiligten- } \\
& \text { analyse }\end{aligned}$ & $\begin{array}{l}\text { 3. Feststellung } \\
\text { fallübergrei- } \\
\text { fender The- } \\
\text { menfelder }\end{array}$ \\
\hline
\end{tabular}




\begin{tabular}{|c|c|c|c|}
\hline $\begin{array}{l}\text { Gliederung } \\
\text { methodisches } \\
\text { Vorgehen }\end{array}$ & Inhalte & Methoden & $\begin{array}{l}\text { Handlungs- } \\
\text { schritte }\end{array}$ \\
\hline $\begin{array}{l}\text { 3. Entwicklung eines } \\
\text { Zielsystems }\end{array}$ & $\begin{array}{ll}- & \text { Zielsystem erarbeiten } \\
- & \text { Ziele für Kooperati- } \\
& \text { on; Ziele für das Netz- } \\
& \text { werk } \\
- & \text { Rückbindung mit } \\
& \text { operativer Ebene }\end{array}$ & $\begin{array}{l}\text { Entwicklung } \\
\text { eines Zielsys- } \\
\text { tems (z. B. } \\
\text { „Zielorien- } \\
\text { tierte Projekt- } \\
\text { planung“) }\end{array}$ & 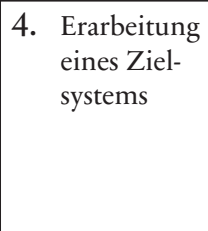 \\
\hline $\begin{array}{l}\text { 4. Dienstleistungs- } \\
\text { ketten/Nebenpro- } \\
\text { dukte }\end{array}$ & & & \\
\hline $\begin{array}{l}\text { 4a. Dienstleistungs- } \\
\text { ketten }\end{array}$ & $\begin{array}{l}\text { Für eine „Produktli- } \\
\text { nie“: Optimierte Ver- } \\
\text { sorgung und Abläufe } \\
\text { mit fachlichen Stan- } \\
\text { dards, Schnittstellen- } \\
\text { definitionen und Ver- } \\
\text { antwortlichkeiten auf } \\
\text { Basis der Rahmenzie- } \\
\text { le } \\
-\quad \text { Kooperationsverein- } \\
\text { barungen zur Absi- } \\
\text { cherung }\end{array}$ & $\begin{array}{ll}- & \text { Matrix } \\
- & \text { Ablaufdia- } \\
& \text { gramm } \\
- & \text { Kooperations- } \\
& \text { vereinbarun- } \\
& \text { gen }\end{array}$ & \begin{tabular}{|rl} 
5a. & Entwick- \\
& lung von \\
& Dienstleis- \\
& tungs-ket- \\
& ten \\
5b. & Abschluss \\
& von Koope- \\
& rations-ver- \\
einbarun- & gen
\end{tabular} \\
\hline 4b. „Nebenprodukte“ & $\begin{aligned}- & \text { Angebote } \\
- & \text { Projekte (je nach } \\
& \text { Grad der Kooperati- } \\
& \text { on) } \\
- & \text { Standards/Verfahren } \\
- & \text { Information/ } \\
& \text { Austausch/Verstehen } \\
& \text { (Zielgruppe, beteilig- } \\
& \text { te Organisationen) }\end{aligned}$ & & \\
\hline $\begin{array}{l}\text { 5. Überprüfung und } \\
\text { Verstetigung }\end{array}$ & & & \\
\hline $\begin{array}{l}\text { 5a. Entwicklung fall- } \\
\text { bezogener Dienst- } \\
\text { leistungskette in } \\
\text { der Fallarbeit }\end{array}$ & $\begin{array}{l}\text { Erprobung der Dienstleis- } \\
\text { tungsketten in der Fallar- } \\
\text { beit }\end{array}$ & $\begin{array}{l}\text { - Fallkonferen- } \\
\text { zen }\end{array}$ & \begin{tabular}{|l} 
6. Entwick- \\
lung und \\
Überprü- \\
fung fallbe- \\
zogener \\
Dienstleis- \\
tungskette \\
in der Fallar- \\
beit
\end{tabular} \\
\hline
\end{tabular}




\begin{tabular}{|c|c|c|c|}
\hline $\begin{array}{l}\text { Gliederung } \\
\text { methodisches } \\
\text { Vorgehen }\end{array}$ & Inhalte & Methoden & $\begin{array}{l}\text { Handlungs- } \\
\text { schritte }\end{array}$ \\
\hline $\begin{array}{l}\text { 5b. Verstetigung der } \\
\text { Netzwerkarbeit }\end{array}$ & \begin{tabular}{|l|} 
Reziprozität \\
Regelmäßigkeit und Dau- \\
erhaftigkeit \\
Architektur \\
Prüfung und Modifikati- \\
on der Leistungsmodule
\end{tabular} & $\begin{array}{l}\text { - } \text { Regeln und } \\
\text { Vereinbarun- } \\
\text { gen }\end{array}$ & $\begin{array}{l}\text { 7. Festlegung } \\
\text { der Struktu- } \\
\text { ren der } \\
\text { Netzwerkar- } \\
\text { beit }\end{array}$ \\
\hline $\begin{array}{l}\text { 5c. Dokumentation } \\
\text { und Evaluation }\end{array}$ & 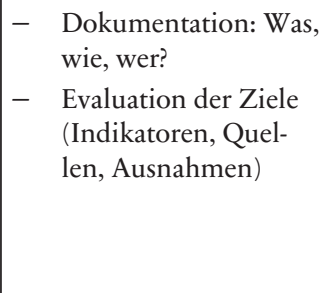 & $\begin{array}{ll}- & \begin{array}{l}\text { Dokumentati- } \\
\text { onsinstru- }\end{array} \\
\text { mente }\end{array}$ & $\begin{array}{l}\text { 8. Dokumenta- } \\
\text { tion und } \\
\text { Evaluation }\end{array}$ \\
\hline
\end{tabular}

\subsection{Methodisches II: Organisationsübergreifende Dienstleistungsketten als Instrument und Ergebnis von Produktionsnetzwerken}

Im Zuge der Arbeit in Produktionsnetzwerken werden Verbindungen zwischen den Leistungen und Angeboten der am Netzwerk beteiligten Akteure deutlich. Über die Definition von Schnittstellen und die Entwicklung von „Brücken“ werden idealtypisch die Konturen eines organisationsübergreifenden Geflechts von Leistungen und Angeboten erkennbar, das im Folgenden als „Dienstleistungskette“ bezeichnet wird. ${ }^{32}$ Diese Dienstleistungsketten können einerseits als Instrument genutzt werden, um die Beziehungen zwischen den am Netzwerk beteiligten Akteure visualisiert darzustellen, gleichzeitig sind sie ein Resultat der Arbeit, wenn sie nämlich ggf. stabilisiert durch Kooperationsvereinbarungen - eine „Richtschnur" für die Kooperation im Einzelfall abgeben. Der Prozesscharakter von Dienstleistungen ist Ausgangspunkt von Überlegungen darüber, wie diese systematisch zustellen sind und eine Verknüpfung in Form von Dienstleistungsketten gedacht werden kann. Darauf folgen Vorschläge zur praktischen Gestaltung von Visualisierungen. Abschließend wird der Zusammenhang von Dienstleistungsketten und Einzelfällen diskutiert.

32 Obwohl es sich weniger um eine „Kette“, als vielmehr um ein „Diadem“ handelt. 


\subsubsection{Das Konzept der „Dienstleistungskette“}

Die Konzeption der Dienstleistungskette greift Gedanken des Prozessmanagements auf (vgl. z. B. Gaitanides 1992; Wilhelm 2007) und nutzt Visualisierungen aus der einschlägigen Literatur. Allerdings sind drei wesentliche Grundlagen immer wieder zu berücksichtigen:

- Da personenbezogene Dienstleistungen immer eine Form von Ko-Produktion von Anbietenden und Benutzern sind, muss die Benutzerperspektive in die Konstruktion von Dienstleistungsketten einbezogen werden. Wird dies verfehlt, droht eine technokratische Verknüpfung von Leistungsangeboten und Leistungserbringung, die aber die Adressatinnen und Adressaten verfehlt und deshalb letztlich scheitern muss.

- Soziale Dienstleistungen werden auch im Hinblick auf politisch gewünschte Wirkungen erbracht bzw. finanziert. Die an einer Dienstleistungskette beteiligten Organisationen verfolgen aber unter Umständen in dieser Hinsicht unterschiedliche Interessen, die sich im ungünstigsten Fall widersprechen können. Somit hat die Konstruktion eines auf dem Papier idealen übergreifenden Leistungsprozesses immer auch eine politische Komponente, die nicht vernachlässigt werden darf.

- Aus diesem Grund basiert eine funktionstüchtige Dienstleistungskette, die gemeinsame Leistungsprozesse strukturiert, nicht nur auf den Leistungsangeboten der beteiligten Organisationen als hervorbringende Strukturen, sondern ebenso auf den Strukturen eines gemeinsam getragenen Produktionsnetzwerks. Hier können die Interessen der einzelnen Organisationen „moderiert“ werden.

Diese Grundlagen gilt es bei der Konstruktion von Dienstleistungsketten systematisch zu bedenken. Außerdem muss differenziert werden zwischen einer Dienstleistungskette, die für jeden einzelnen Fall entworfen werden kann und einer Dienstleistungskette, die im Sinne der Prozessorganisation für eine Vielzahl ähnlicher Fälle ,idealtypisch“ entwickelt wurde. Letztere baut auf der Analyse von Einzelfällen auf - methodisch kann hier auf das Verfahren beim Aufbau von Produktionsnetzwerken zurückgegriffen werden. (vgl. Reis et al. 2016) Es gibt also für bestimmte, ähnlich gelagerte Fallkonstellationen jeweils eine idealtypische Dienstleistungskette, die in der konkreten Fallarbeit genutzt werden kann. Dabei sollte aber niemals schematisch vorgegangen werden, vielmehr gilt es - ganz im Sinne der KoProduktion - die Besonderheiten jeden Einzelfalls angemessen zu berücksichtigen. 


\subsubsection{Prozessorganisation}

In den 1990er-Jahren wurde sowohl in privaten Unternehmen als auch öffentlichen Verwaltungen versucht, die Arbeits- und Betriebsorganisation so umzustrukturieren, dass eine Konzentration auf „Kernprozesse“ erfolgt. Hintergrund waren die Kostenstrukturen der Produktion unter dem Regime funktionaler Arbeitsteilung: Die Auslagerung aller planerischen Aktivitäten in Dienstleistungsstäbe führte zu einem extrem hohen Anteil indirekter (d. h. nicht auf die Produktionsprozesse direkt zurechenbarer) Kosten an den Gesamtkosten. Bestrebungen einer stärkeren Dezentralisierung und Aufwertung der direkten Bereiche, d. h. der operativen Einheiten (unter dem Slogan der „lean production“), zielten deshalb u. a. darauf ab, die Gemeinkostenbereiche neu zu gestalten, nämlich in sachlich zusammengehörige kostenstellenübergreifende Prozessketten zu überführen. Es fand eine Fokussierung auf die Geschäftsprozesse als Ursache und Begründung des Kostenanfalls statt.

Die Produktion wurde seither auch organisatorisch stärker als in der Vergangenheit als Prozess gestaltet, mit klarer Ausrichtung auf die Prozessteile, die unmittelbar „wertschöpfend“ sind, d. h. für die Kundinnen und Kunden einen Nutzen- oder Wertzuwachs erzeugen, für den sie einen Preis zahlen. Im Bereich der materiellen Produktion sind dies die Bereiche Konstruktion, Produktion, Montage, Vertrieb, Marketing sowie Kundendienst. Unterstützend sind z. B. die Prozesse Infrastruktur, EDV-Einsatz, Personalwirtschaft, Beschaffung, die übergreifend für alle Prozessteile tätig sind. Der dieser Neugestaltung zugrundeliegende Leistungsprozess „richtet sich (...) an der für den externen Bürger beziehungsweise Kunden nützlichen Leistung aus und soll diese so wirtschaftlich und nutzbringend wie möglich erbringen. Prozesse, die dazu direkt oder indirekt nichts beizutragen vermögen, sind wirtschaftlich nicht zu legitimieren. Dabei werden alle für die erfolgreiche Erfüllung eines Prozesses erforderlichen unternehmens- beziehungsweise verwaltungsinternen Aktivitäten und Funktionen betrachtet" (Naschold et al. 1996: S. 39). Rein technisch hat die Betrachtung von Leistungsprozessen in Unternehmen und Verwaltungen eine lange Tradition. So wird in der Verwaltungslehre seit Langem zwischen der Aufbau- und der Ablauforganisation unterschieden. Erstere gibt die Hierarchie wieder (vgl. Organigramme) und letztere erteilt Auskunft über die Gestaltung von typischen Produktions- und Leistungsprozessen in Organisationen. Diese Prozesse können quer zur Aufbauorganisation verlaufen.

Eine Prozessbetrachtung beginnt mit einem komplexen Gesamtprozess, der dann in Form einer Prozessanalyse systematisch in Teilprozesse „zer- 
legt" wird. Hinter der Idee der Prozessorganisation steht die Notwendigkeit einer möglichst flexiblen Koordination der Leistungsprozesse in Unternehmen und Verwaltungen. Hierzu muss definiert werden, welche funktional getrennten, aber zusammengehörigen Leistungen miteinander zu einem Prozess verknüpft werden können. Übergänge an den Schnittstellen werden definiert und so Brüche identifiziert. Ein Leitsatz von Prozessorganisationen lautet: Die Konzentration liegt auf den Nutzen generierenden Leistungsprozessen. Diese stehen im Fokus, denn sie ermöglichen die Erfüllung der Anforderungen bzw. der gewünschten Ergebnisse („Primärprozesse“). Führungsprozesse (Leitungstätigkeit) und Unterstützungsprozesse (z. B. die Wartung von Computern, die Beschaffung von Sachmitteln, die Abrechnung von Honoraren) sind nicht leitend, stehen aber in einem Abhängigkeitsverhältnis zu den gewünschten Ergebnissen („Sekundärprozesse“). Deshalb werden auch sie analysiert und koordiniert, da es sonst zu Störungen der Leistungsprozesse kommen kann. Ein explizites Anliegen der Prozessorganisation ist deren kontinuierliche Verbesserung. Die Beschreibung und Festlegung der Prozesse dient als Grundlage einer regelhaften Überprüfung der Prozessqualität (vgl. Brüggemann/Bremer 2012). Werden Leistungsprozesse standardisiert und formalisiert, erhalten die hierfür erstellten Anleitungen (z. B. Qualitätshandbücher) den Status formalisierter Programme. Damit wird manifest, dass die Prozessorganisation (und so auch die „Dienstleistungsketten“) auf „typische“ Leistungen und Leistungsprozesse zielt. Individuelle Leistungen, d. h. „Einzelfälle“, werden im Zuge der Prozessorganisation bearbeitet, Dienstleistungsketten bilden hierfür den Rahmen.

Ausschlaggebend für die Produktivität der Übertragung des Konzepts der Leistungskette vom Unternehmenssektor auf die Produktion personenbezogener Dienstleistungen im öffentlichen Erbringungskontext ist dessen Grundgedanke: Die Ausrichtung aller Teilprozesse auf die Stiftung eines möglichst großen Nutzens bei der Kundschaft, also die eindeutige Zielorientierung auf eine optimale und effektive Leistung im Einzelfall. Nach Maßgabe dieser Zielsetzung sind die einzelnen Prozessschritte zu bewerten und notfalls neu zu gestalten. Somit wird das Konzept der Dienstleistungskette für die konkrete Ausgestaltung von im öffentlichen Kontext erbrachten Dienstleistungen, die den Gedanken der Kundenorientierung realisieren sollen, ein wichtiges Handlungsinstrument.

Die Leistungskettenbetrachtung kann weiter differenziert werden, denn auch Teilprozesse, für die Einzelne oder Gruppen verantwortlich sind, können wiederum als „Leistungsketten“ analysiert werden, d. h. der Teilprozess wird in einzelne, miteinander verknüpfte Prozessschritte zerlegt. 


\subsubsection{Organisationsübergreifende Dienstleistungsketten}

Die (Re-)Konstruktion von organisationsübergreifenden Dienstleistungsketten kann sich sowohl auf die deskriptive als auch die präskriptive Variante stützen. Einerseits werden bestehende Leistungsprozesse analysiert (deskriptiv), andererseits können neue Leistungsprozesse als Dienstleistungskette konstruiert werden (präskriptiv). Für die Analyse der Ist-Situation ist ein deskriptives Vorgehen sinnvoll. Es kann z. B. im Rahmen von Fallanalysen eingesetzt werden, um zu rekonstruieren, wie die Aktivitäten der am Fall Beteiligten miteinander verknüpft, wie typische Abläufe ausgestaltet sind oder Organisationsroutinen aussehen. Auf der Basis einer IstAnalyse der Abläufe kann dann mit der Neugestaltung von Prozessen begonnen werden. Hier kann die Konstruktion von Dienstleistungsketten die Planungen von Leistungsprozessen konkretisieren. Wie oben bereits erwähnt, ist immer zu berücksichtigen, dass

- sich Dienstleistungsketten zwar auf Einzelfälle stützen, aber in ihrer Konstruktion von diesen abstrahieren,

- es nicht nur eine, sondern stets mehrere Dienstleistungsketten geben wird - je nach Menge der „typischen“ Fallkonstellationen.

Auch hier sind für das konkrete Vorgehen verschiedene Varianten denkbar. Im Zuge der Reform des Fürsorgesystems der USA haben Corbett/ Noyes ein Verfahren entwickelt, das konsequent präskriptiv ansetzt und hierfür Erkenntnisse des strategischen Managements nutzt (vgl. Corbett/ Noyes 2006, vgl. Abschnitt 4.3.3)). Ausgangspunkt ist die Festlegung der Zielgruppe und die Beschreibung ihrer Ausgangslage. Eine Skizzierung der Zielpopulation und ihrer wesentlichen Problemlagen wie Ressourcen stellt eine wesentliche Vorbereitung für weitere Planungen dar. Hierzu kann die Methode der Fallrekonstruktion und Fallanalyse genutzt werden, die im Rahmen des Projekts angewandt wird. Auf der Basis der Verständigung über das für die ausgewählte Zielgruppe anzustrebende Grundsatzziel werden Rahmenziele und die (gewünschten) Ergebnisse festgelegt. Es muss definiert werden, was im Einzelnen geleistet werden soll. Hintergrund hierfür ist die Entwicklung eines Zielsystems, in dem Rahmen- und Ergebnisziele aus dem Grundsatzziel abgeleitet werden. Hierfür gibt es Techniken, die im Rahmen des Projekts vermittelt wurden. Wenn die Zielgruppenpopulation und die für diese erwünschten Ergebnisse (Grundsatz-, Rahmen- und Ergebnisziele) erst einmal bestimmt worden sind, besteht der nächste Schritt darin, klar zu formulieren, wie bzw. durch welche Aktivitäten diese Ergebnisse erreicht werden können. 
Das größte Hindernis bei der Umsetzung von Dienstleistungsketten liegt darin, kurzfristige taktische Entscheidungen („technische Lösungen“) mit langfristigem strategischem Denken zu verwechseln. Wer eine Vision von Kooperation realisieren möchte, wird - so Corbett/Noyes - schnell dazu verführt, anzunehmen, dass bekannte Lösungswege bzw. Maßnahmen zu den erwünschten Ergebnissen führen könnten, ohne genau darüber nachzudenken, ob diese Lösungen wirklich geeignet sind, auch langfristig wirksame Strategien zu realisieren, d. h. Arbeitsweisen und Kooperationsstrukturen zu verändern. Die Tragfähigkeit dieser Strategie erweist sich, wenn die Akteure die Ergebnisse aus der Perspektive der adressierten Personen ihrer Leistungen betrachten. Die Schlüsselfrage lautet: Wie sieht dieses neue System aus der Perspektive der Zielgruppe aus („line of sight“-Perspektive, vgl. Abschnitt 4.3.3)? Werden sich die neuen Leistungen und Prozesse qualitativ von dem unterscheiden, was sie gegenwärtig erleben? Um diese Frage voll und ganz beantworten zu können, muss man sich darauf konzentrieren, was Adressatinnen und Adressaten wahrscheinlich erleben werden, wenn jeder wichtige Schritt in dem neuen System „maßgeschneidert" ist. Wie werden Mitglieder der Zielgruppenpopulation von diesem System erfahren? Was genau geschieht, wenn sie zur Eingangstür hereinkommen? Was danach? Obwohl man nicht mit Sicherheit wissen kann, was eine gewisse Person oder eine Familie in dem neuen System erleben wird, sollte es möglich sein, Szenarien dafür zu entwerfen, was durchschnittliche Personen oder Familien erleben könnten, wenn die Planungen so greifen würden wie erhofft. Zentrale Prozesselemente sind hierbei: Programmbewusstsein (Signalwirkung und Reichweite), die Gestaltung des ,intake“ (Antragstellung, Diagnose und Weiterverweisen an wichtige Dienstleistende), das Erbringen von Leistungen und fortlaufendes Case Management (Verlaufsbeobachtung und Problemlösung) sowie die Strategien der „Beendigung“ (Erfolgsermittlung und Fortschreibung).

Bei der konkreten Ausgestaltung der Leistungserbringung in einer Dienstleistungskette ist der Unterschied zwischen den verschiedenen Formen der systematischen Verbindung von Arbeitsprozessen und den damit verknüpften Koordinationsproblemen zu beachten:

- Parallele Arbeitsprozesse, z. B. Durchführung einer Vorsorgeuntersuchung und Beratung über vorhandene Ansprüche auf materielle Leistungen. Hier gibt es keinen oder nur einen geringen Koordinationsaufwand im Hinblick auf Inhalte bzw. Zeiten.

- Sequentielle Arbeitsprozesse, d. h. ein Arbeitsprozess muss beendet sein, damit der nächste beginnen kann, z. B. hintereinander geschaltete Vorsorgeuntersuchungen (U1, U2, U3 etc.) oder Unterrichtsstunden in 
der Schule. Hier gibt es einen geringen Koordinationsaufwand im Hinblick auf Inhalte, unter Umständen jedoch einen hohen Aufwand bezüglich der Zeitplanung - insbesondere, wenn mehrere Arbeitsprozesse koordiniert werden müssen (Erstellung eines Stundenplans).

- Verzahnte Arbeitsprozesse, d. h. solche, die aufeinander aufbauen bzw. Arbeitsprozesse, bei denen der zweite auf bestimmte Resultate des ersten angewiesen ist, z. B. Arbeitsvermittlung erst und nur dann, wenn eine bestimmte Qualifizierung erreicht ist. Hier existiert hoher Koordinationsaufwand im Hinblick auf Inhalte (Definition des erreichten Ergebnisses des ersten Arbeitsprozesses) und Zeiten (tatsächlicher Beginn des zweiten Arbeitsprozesses).

Je nachdem, welche Form der Verknüpfung notwendig ist, um die gewünschten Ergebnisse zu erzielen, können die Arbeitsprozesse lockerer oder müssen straffer miteinander verbunden und entsprechend gesteuert werden. Sobald die „line of sight“ aus Zielgruppensicht etabliert ist, wird es notwendig, zu bedenken, was genau die am Netzwerk beteiligten Organisationen tun müssen, um diesem neu entworfenen Pfad zu folgen. Welche Veränderungen müssen in der institutionellen Praxis, Verwaltung und Politik getroffen werden, um eine Umgestaltung der Erfahrungen der Zielgruppe zu unterstützen? Welches Personal, welche Fähigkeiten, Kenntnisse, Gelder und welcher Raum sind nötig, damit das System für die Zielgruppe funktioniert? Ist der richtige Mix an Ressourcen an den richtigen Orten zur richtigen Zeit und im richtigen Umfang zugänglich? Welche zentralen Abläufe gibt es im neuen Modell? Was muss an bestehenden Abläufen modifiziert werden, um sicherzustellen, dass diese auch realisiert werden? Schlussendlich müssen die Inputs und die platzierten Ressourcen, die Aufgabenabfolge, Tätigkeiten und Entscheidungen sowie die erwarteten Ergebnisse und Erträge einer durchgängigen Logik folgen. Wenn das Modell bspw. anspruchsvolle Diagnosen erfordert, muss man sich fragen, ob die notwendige Fachkompetenz für solche Bewertungen bei den verschiedenen Akteuren vorhanden ist (ein Ressourcen- bzw. Inputproblem). Hieran schließt der Schritt an, die geplanten Abläufe mit dem institutionellen Milieu zu vergleichen, in dem sie realisiert werden sollen. Hier stellt sich insbesondere folgende Frage: Welche Hindernisse und Herausforderungen können bei der Implementierung der beabsichtigten Maßnahmen aus dem grundlegenden institutionellen Milieu entstehen? Die Beantwortung dieser Frage schließt die Überprüfung mit ein, ob Leitung, institutionelles System und Organisationskultur die geplanten Abläufe tatsächlich unterstützen können. 
- Bezogen auf die Leitung: Sind die grundlegenden Annahmen über die politische Unterstützung realistisch? Sind die politischen Kompetenzen vorhanden, die gewünschten Veränderungen auch durchzuführen?

- Bezogen auf das institutionelle System: Sind Programmplanung und -verantwortung ergebnisorientiert? Sind Schulungsmaßnahmen bedarfsgesteuert, sachdienlich und aktuell? Ist das informationstechnologische Umfeld bezogen auf die Erneuerungen leistungsfähig? Ist das Finanzmanagement flexibel und verlässlich?

- Bezogen auf die Organisationskultur: Ist das Personal von einer gemeinsamen organisatorischen Vision überzeugt? Begreift es seine führende Rolle bei der Realisierung der Ergebnisse? Kann eine kontinuierliche Verbesserung erwartet werden? Wissen die Mitarbeitenden, was von ihnen erwartet wird?

Für die Umsetzung der Perspektive einer „line of sight“ ist es wichtig, die geplanten Leistungsprozesse mit Blick auf das existierende Umfeld zu überprüfen. Wenn der Plan letztendlich für unrealistisch befunden wird, muss er entweder geändert oder es müssen bedeutende Anstrengungen unternommen werden, um das bestehende operative Umfeld umzugestalten und so die intendierten Änderungen zu ermöglichen.

\subsubsection{Visualisierungen}

\subsubsection{Grundsätzliches}

Um die Analyse inner- und interorganisatorisch zu unterstützen, wurden in der Literatur verschiedene Formen der Visualisierung entwickelt (vgl. Liebelt 1992; Wilhelm 2007; Miebach 2009; Schiersmann/Thiel 2014). Die Darstellung kann dabei mehr oder weniger stark formalisiert werden und auf unterschiedlichen Abstraktionsebenen erfolgen. Ein Darstellungsmittel sind „Prozesslandkarten“. An ihnen kann abgelesen werden,

- „welche Prozesse im Unternehmen vorhanden sind,

- welche Beziehungen zwischen (interner) Kundschaft und Lieferanten durch die Prozesse gegeben sind und

- über welche Prozesse das Unternehmen mit seiner (externen) Kundschaft und Lieferanten verbunden ist" (Wilhelm 2007: S. 34).

Prozesslandkarten geben die „innere Logik“ der Bearbeitung bzw. Dienstleistungserbringung wieder (vgl. z. B. Allweyer 2005: S. 8). Eine Ausdifferenzierung kann in Form von Fluss- bzw. Ablaufdiagrammen vorgenommen werden. Diese Flussdiagramme geben einen detaillierten Überblick 
über Abläufe. Eine Version der Darstellung von Prozesslandkarten, nämlich die der Business Process Management Notation (BPMN)(C), stellen Schiersmann/Thiel vor. Sie ist vereinfacht und erlaubt dadurch die Darstellung komplexerer, z. B. abteilungs- oder organisationsübergreifender Prozesse. Bemerkenswert in der Darstellung sind die „swim lanes“, die es erlauben, auf einfache Weise Prozesse abzubilden, die Organisationseinheiten bzw. Organisationen übergreifen (vgl. Schaubild 9).

Schaubild 9: Übergreifender Prozess in der BPMN@

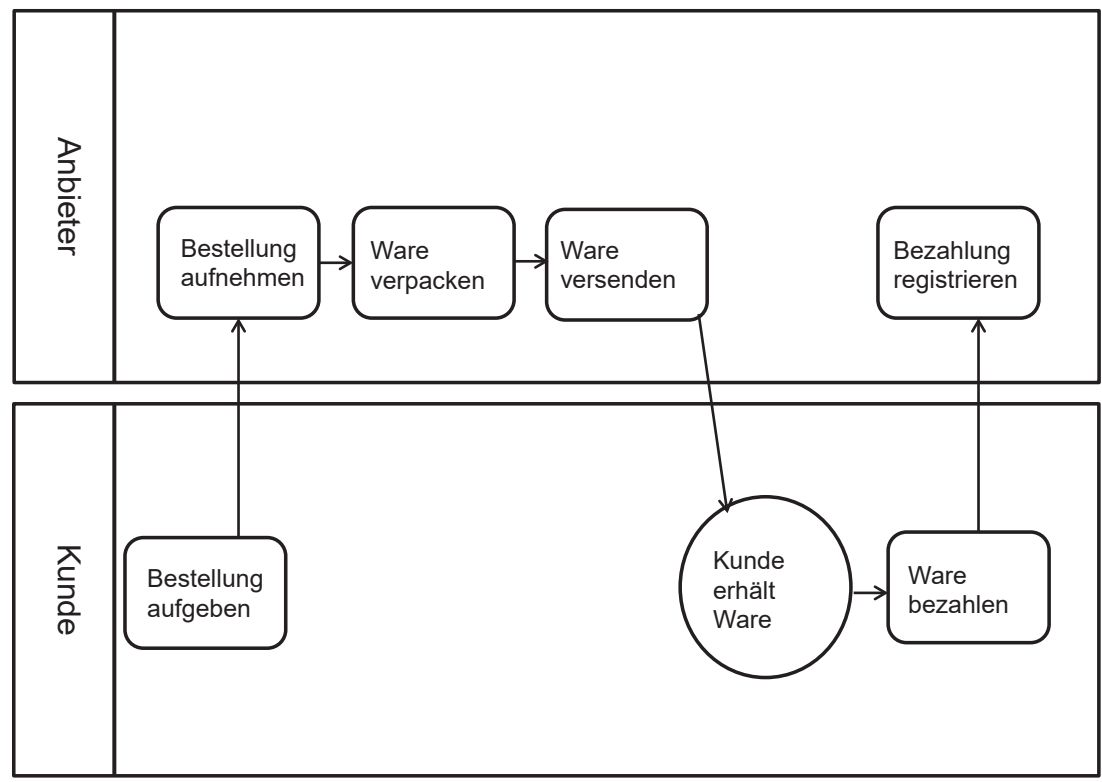

(eigene Darstellung, angelehnt an Schiersmann/Thiel 2014: S. 344)

Diese Prozesslandkarten und Ablaufdiagramme dienen zunächst der Beschreibung von Abläufen, haben also deskriptiven und keinen präskriptiven Charakter. Gleichwohl können sie genutzt werden, um neu konzipierte Dienstleistungsketten grafisch darzustellen.

\subsubsection{Hinweise zur praktischen Gestaltung}

In den hier wiedergegebenen Grafiken werden die Prozesse in vielfältiger Weise visualisiert, d. h. grafisch dargestellt. Bei all diesen Visualisierungen, 
ob in Form einer Prozesslandkarte oder eines Flussdiagramms kommt es darauf an, komplexe Prozesse möglichst einfach darzustellen, ohne wesentliche Informationen zu unterschlagen.

Deshalb müssen zwei Probleme gelöst werden:

- Die Entscheidung über den Grad der Detaillierung. Gerade bei Flussdiagrammen, die ja einzelne Prozesse differenziert darstellen, stellt sich die Frage der Detailliertheit der Darstellung. Wenn jeder Handgriff visualisiert wird, wird ein Flussdiagramm schnell zu einer ästhetischen Übung ohne praktischen Nutzen, werden komplexe Prozesse zu sehr verdichtet, schwindet der Informationswert. Deshalb ist es sinnvoll, zunächst die grundlegenden Abläufe zu identifizieren und in ihrer Chronologie festzuhalten, danach eine Prozesslandkarte zu erstellen, um anschließend zu entscheiden, welche Prozessschritte für den Gesamtprozess von hoher Bedeutung sind. Diese können dann in einem Flussdiagramm detailliert dargestellt werden. Dies empfiehlt sich gerade an den „Schnittstellen“ zwischen zwei Organisationseinheiten.

- Die Entscheidung über die Art der Visualisierung. Es gibt verschiedene Symbolsysteme, die für die Visualisierung von Prozessen angeboten werden (vgl. Schiersmann/Thiel 2014). Gerade für die gemeinsame diskursive Erstellung von Dienstleistungsketten empfiehlt sich eine möglichst einfache Darstellungsform, die mit weit verbreiteten Softwareprogrammen (wie z. B. PowerPoint) zu realisieren sind. In Schaubild 10 findet sich eine am Modell der BPMN angelehnte Darstellung. Sie hat den Vorteil, dass sie durch die „swim lanes“ unterschiedliche Organisationen bzw. Organisationseinheiten darzustellen erlaubt, durch die ein einzelner Prozess verläuft. Allerdings sind die einzelnen Elemente der BPMN nicht einfach über PowerPoint darzustellen, weswegen hier eine Kombination mit den weit verbreiteten Notationen der Flussdiagramme vorgeschlagen wird.

Es ist auf alle Fälle sinnvoll, eine einheitliche Darstellungsform zu wählen. „Bunte Bilder“ haben sicher ihre Ästhetik, meist aber mehr illustrativen als analytischen Wert. Ein weiterer Aspekt ist die Beschränkung der Information über das tatsächliche Prozessgeschehen. Eine Abbildung des Ablaufs einer Beratung kann auf die Darstellung der Beratungsinhalte getrost verzichten, solange sie keine direkten Auswirkungen auf die Verläufe haben. Genauso redundant sind Informationen über Kooperationsbeteiligte, diese können problemlos in einem Anhang „mitgeliefert“ werden.

Mit dem im Folgenden wiedergegebenen Beginn einer Dienstleistungskette in Form einer Prozesslandkarte soll exemplarisch gezeigt werden, wie die Unterstützungsleistungen für junge Migrantinnen mit Kind(ern) im 
Vorschulalter konkret ausgestaltet sein können (vgl. Schaubild 10). Der Prozess beginnt mit der Vorsprache einer Migrantin im Jugendmigrationsdienst. Der JMD beginnt nach einer ersten Beratung, in der sich die Komplexität des Falls bereits zeigt, das Case Management mit einem Assessment (Bestandsaufnahme). Hierauf folgt der Übergang in die Hilfeplanung. Bestandteile des daraus resultierenden Förderplans ist der Verweis an das Jobcenter sowie das Jugendamt, um die Kinderbetreuung zu sichern. Entsprechend sollen die Frauen das Jobcenter aufsuchen und beim Jugendamt einen Antrag auf einen Platz in einer Kindertageseinrichtung sowie ggf. anschließende Betreuung in Tagespflege für ihr Kind stellen. Wenn starke psychische Belastungen vorliegen, besteht die Möglichkeit einer therapeutischen Betreuung. Im Jobcenter werden erneut ein Assessment (dort „Profiling“ genannt) sowie eine Hilfeplanung durchgeführt und eine Eingliederungsvereinbarung abgeschlossen. Gegenstand der Eingliederungsvereinbarung ist der Besuch eines Integrationskurses.

\section{Schaubild 10: Beginn einer Dienstleistungskette im Flussdiagramm}

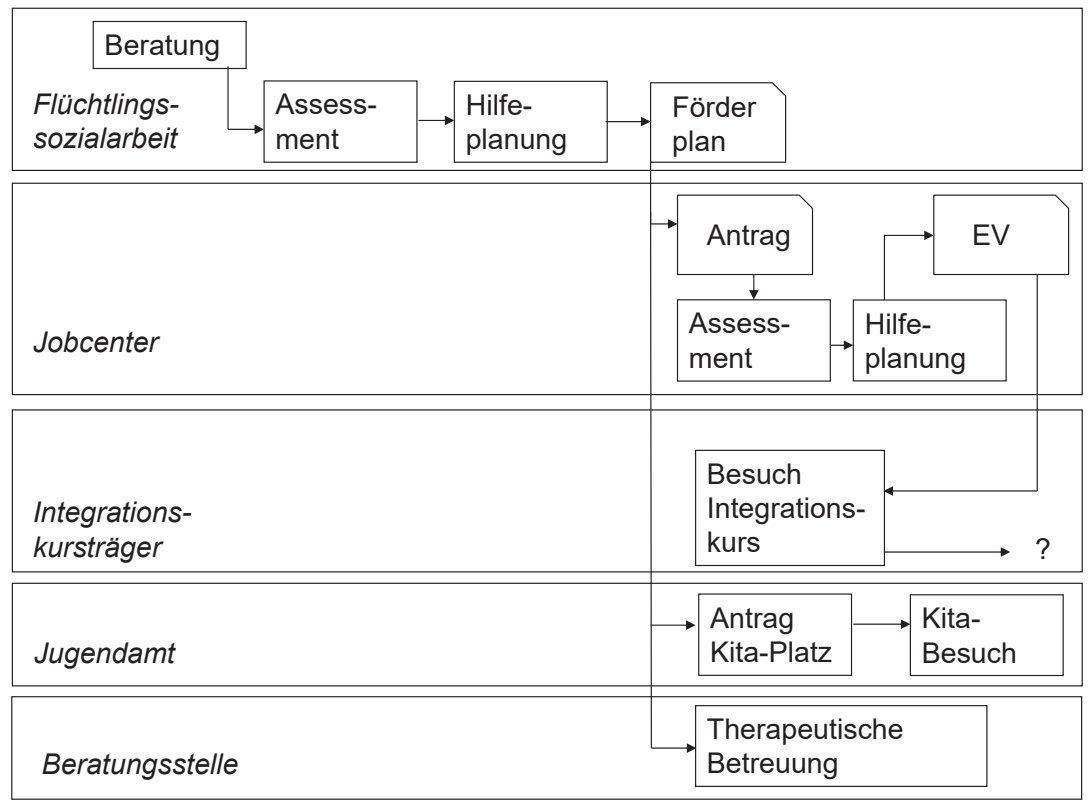

(eigene Darstellung) 


\subsubsection{Dienstleistungsketten und integrierte Leistungen}

Oben wurde das Konzept der Dienstleistungskette entwickelt und gezeigt, dass diese Ketten sich auf „typische“ Konstellationen beziehen, in denen bestimmte Dienstleistungen erbracht und kombiniert werden. Dienstleistungsketten abstrahieren deshalb immer von individuellen „Fällen“, nehmen deren allgemeine Merkmale auf und sehen von Individuellem ab.

Wie bereits in Abschnitt 4.6 ausführlich diskutiert wurde, können konkretes Fallverstehen und verallgemeinernde Fallrekonstruktion methodisch voneinander getrennt werden - um die strukturellen Aspekte eines Falls zu bearbeiten, muss nicht der subjektiv gemeinte Sinn bis in alle Verästelungen erschlossen werden. An dieser Stelle zeigt sich die Rückkopplung zum Case Management. Dieses hat die Aufgabe, im Prozessschritt „Assessment" die individuelle Situation seiner Klientinnen und Klienten zu erschließen und auf „allgemeine“ Problemkonstellationen und die hierfür (z. B. als Dienstleistungskette) entwickelten Lösungswege zu beziehen. Hierfür ist „Fallverstehen“ im oben entwickelten Sinne elementar; sollte es sich als notwendig erweisen, sind die „Lösungswege“ flexibel auf die individuelle Situation zuzuschneiden - was unter Umständen eine intensive Beratungsarbeit erfordert. Case Management ist also nicht zuerst „Management" der optimalen Unterstützungsangebote (die immer schon über das „Allgemeine“ definiert sind), sondern findet seinen Kern in der Transformation des Besonderen ins Allgemeine, ohne dass das Besondere verloren geht. Um dies zu bewerkstelligen, ist unter Umständen eine zumindest mittelfristige, aber intensive Beratungsarbeit notwendig - nämlich dann, wenn die Klientinnen und Klienten nicht selbst in der Lage sind, Angebote, die z. B. über eine Dienstleistungskette zur Verfügung gestellt werden, so an ihre besondere Bedarfslage anzupassen, dass sie tatsächlich hilfreich sind.

Case Management steuert somit eine auf den Einzelfall bezogene „integrierte Leistung“, die auf der Basis der Hilfeplanung (und ggf. gemeinsamer Fallkonferenzen) von unterschiedlichen Leistungsanbietende erbracht wird. Optimalerweise stützt sich diese integrierte Leistung auf Dienstleistungsketten, die für bestimmte Fallkonstellationen auf der Basis gemeinsamer Planung entwickelt wurden. Letztendlich muss ein komplexes Dienstleistungsangebot vier Elemente umfassen:

- Leistungsangebote, die von einzelnen Organisationen auf der Basis bereitgestellter Ressourcen (Inputs) erbracht werden - hierdurch werden Teilhabemöglichkeiten geschaffen. 
- Ein Produktionsnetzwerk, das diese Leistungsangebote strukturell vernetzt - hierdurch werden Teilhabemöglichkeiten auch in komplexen Fällen verbessert.

- Dienstleistungsketten, die auf dieser Basis für verschiedene Fallkonstellationen entwickelt wurden und die die Brücke zu den Einzelfällen schlagen.

- Case Management oder ein analoges Beratungsangebot, das sich auf die bedürftigen Individuen bezieht, deren individuelle Problemlagen erschließt und die Individuen in die Lage versetzt, bereitgestellte Angebote auch für sich zu nutzen (vgl. das Konzept der Teilhabekompetenz, Abschnitt 3.1). Eine wesentliche Funktion des Case Managements ist es dabei, eine integrierte Leistung zu steuern.

Aus der Perspektive des Gesamtprozesses betrachtet, stellen Dienstleistungsketten die Verbindung zwischen der Strukturebene (Leistungsangebote, Produktionsnetzwerke) und der Handlungsebene (Interaktion der Case Manager mit den adressierten Personen) dar. Deshalb müssen sie beides aufnehmen: Die (fach-)politischen Konstellationen, die sich in den Produktionsnetzwerken zeigen und die Perspektive der Benutzer ohne die eine Dienstleistung nicht zu Stande kommt.

\subsection{Methodisches III: Wie werden Netzwerke gesteuert?}

Der Aufbau und die Stabilisierung von Produktionsnetzwerken setzen ein professionell operierendes „Netzwerkmanagement“ voraus. ${ }^{33}$ Dessen Aufgaben bestehen u. a. in der Moderation von Netzwerktreffen, der Evaluation und Dokumentation der gemeinsamen Arbeit, der „Beziehungspflege“ und der Anregung von „Reflexion“ sowie der Vertretung des Netzwerks nach „außen“. Das Netzwerkmanagement muss nicht zwingend von einer einzelnen Person umgesetzt werden, benötigt aber Ressourcen und Kompetenzen.

Im Folgenden wird skizziert, was unter „professionellem Netzwerkmanagement" zu verstehen ist. Ähnlich wie der Begriff des „Netzwerks“ muss der des „Netzwerkmanagements“ präzisiert werden, um für die Analyse

33 Inwieweit zur Erfüllung anderer Netzwerkfunktionen „Netzwerkmanagement“ notwendig ist, ist eine eher empirisch zu beantwortende Frage. Auch muss geklärt werden, welche Funktionen des Netzwerkmanagements für unterschiedliche Konstellationen notwendig erfüllt werden müssen. 
des konkreten Geschehens in Netzwerken fruchtbar gemacht werden zu können. $\mathrm{Zu}$ unterscheiden sind insbesondere:

- Management als Institution und als Funktion sowie

- die unterschiedlichen Funktionen des Netzwerkmanagements.

\subsubsection{Management als Institution und als Funktion}

Der Aufbau und die Verstetigung eines Netzwerks sind die zentralen Aufgaben des „Netzwerkmanagements“. Mit diesem Begriff wird sowohl eine bestimmte institutionalisierte Koordinationsstelle wie auch die Steuerungsfunktion bezeichnet, die von einer oder mehreren Person(en) wahrgenommen wird. Ein stabiles Netzwerk erfordert eine klare institutionelle Anbindung und eine professionelle Koordination. Dies bedeutet jedoch nicht zwangsläufig die Institutionalisierung einer festen „Koordinationsstelle“, vielmehr sind auch flexible Lösungen denkbar (z. B. die wechselseitige Übernahme von Funktionen des Netzwerkmanagements). Aus diesem Grund ist es sinnvoll, zwischen den Funktionen des Netzwerkmanagements einerseits und den Institutionalisierungsformen, in denen diese Funktionen wahrgenommen werden, andererseits zu unterscheiden.

Unabhängig von der Form der Institutionalisierung müssen im Netzwerk die im Folgenden genannten Kompetenzen für eine professionelle Koordination vorgehalten und weiterentwickelt werden.

- Politikfeldübergreifende Fachkenntnisse und Analysetechniken,

- Kenntnisse in Netzwerktheorie und -praxis,

- Steuerungs- und Projektmanagementtechniken,

- Kenntnisse in Verfahren des Qualitätsmanagements (zur Analyse von Leistungsketten),

- Kompetenzen der Verhandlungsführung (Gesprächsführung, Umgang mit schwierigen Beteiligten, Konfliktmanagement),

- Interkulturelle Kompetenzen,

- Kompetenzen für einen sensiblen Umgang mit Entscheidungsebene und operativer Ebene,

- Fähigkeiten zum akteur- und perspektivenübergreifenden Handeln,

- Kenntnisse über die lokalen Kräfteverhältnisse und Kulturen der lokalen Kooperation, u. a. für einen reflektierten Umgang mit der Entscheidung über die Beteiligung der relevanten Akteure bzw. die Ansiedlung des Netzwerks,

- Anerkennung bzw. Autorität in der lokalen Trägerlandschaft (als Einrichtung und als Person). 
Neben diesen Kompetenzen müssen strukturelle Voraussetzungen für den Aufbau stabiler Koordinationsstrukturen gegeben sein:

- Gewährleistung organisatorischer und finanzieller Ressourcen,

- Neutrale und hoch vernetzte Koordination,

- Die Akzeptanz der Koordination sollte im (potenziellen) Netzwerk bereits vor dem offiziellen Beginn des Vorhabens gegeben sein.

Die konkrete Konstruktion des Netzwerks und damit auch die Form der Institutionalisierung von Netzwerkmanagement hängen stark von den örtlichen Verhältnissen ab. Je nach zugewiesener Funktion und Größe wird es nötig sein, mehrere Ebenen zu definieren: Einerseits die Entscheidungsebene und die operative Ebene, andererseits die Ebene eines breiten Netzwerks, in dem sich ein „ideelles Milieu“ entwickeln kann, und - daran anknüpfend - die eines verbindlichen Produktionsnetzwerks. Außerdem kann es sinnvoll sein, zunächst als Ausgangspunkt ein „Kernnetzwerk“ zu konstituieren, das überschaubar und arbeitsfähig ist, und später ggf. Erweiterungen vorzunehmen.

\subsubsection{Funktionen des Netzwerkmanagements}

Sydow und Windeler definieren „Netzwerksteuerung“ inhaltlich über ein Bündel von Aufgaben (vgl. Sydow 1999: S. 295f.; Windeler 2001: S. 44, 250), die in vier zentralen Funktionen zusammengefasst werden:

- Selektion der Akteure, die das Netzwerk bilden bzw. später aufgenommen werden (4.8.2.1),

- Allokation, d. h. Verteilung der Aufgaben, die die Akteure wahrnehmen (4.8.2.2),

- Regulation, d. h. Festlegung von Strukturen und Regeln der Aufgabenwahrnehmung (4.8.2.3),

- Evaluation, d. h. Auswertung und Bewertung der Arbeit des Netzwerks (4.8.2.4) (vgl. Sydow/Windeler 1997: S. 151).

\subsubsection{Selektion: Auswahl der Netzwerkpartnerinnen und -partner}

Im Vorfeld der Bildung eines Netzwerks stehen zwei Aufgaben im Vordergrund:

- Die Entscheidung, welche potenziellen Mitglieder eines Netzwerks angesprochen werden sollten. 
- Die Sensibilisierung für die Interessen- und Motivlage dieser potenziellen Partnerinnen und Partner.

Eine Beteiligtenanalyse kann zeigen, welche Organisationen und Institutionen vor Ort auf die Lebenssituation der Zielgruppe einwirken und damit potenziell in ein Produktionsnetzwerk einbezogen werden sollten (vgl. Abschnitt 4.6.2). Im Fokus des zu bildenden Netzwerks steht ein gemeinsames Problem bzw. Ziel, das unter Umständen aber zu diesem frühen Zeitpunkt noch nicht von allen (künftig) Beteiligten als gemeinsames Problem oder Ziel gesehen wird. Bevor dies in der Konstitutionsphase thematisiert wird, soll zunächst eine möglichst große Bandbreite an Angeboten für die Zielerreichung realisiert werden, d. h. es ist zu entscheiden, welche Organisationen und Institutionen unbedingt in ein Netzwerk einbezogen werden sollten.

Hintergrund hierfür ist das Postulat der Heterogenität eines Netzwerks, um alle Perspektiven der Problemformulierung und -lösung repräsentiert zu haben und die nötige Vielfalt an Kompetenzen im Netzwerk zu versammeln: „Um ein breites Kompetenzspektrum sicherzustellen, benötigen Netzwerke einen möglichst hohen Grad an Heterogenität. Verschiedene Arbeitsfelder und Tätigkeitsschwerpunkte sind im Netzwerk präsent und können zu neuen Tätigkeitsbereichen verknüpft werden" (Borkenhagen u. a.: S. 34). Nach einer ersten Analyse des Feldes werden die Strukturen eines potenziellen Produktionsnetzwerks deutlich, können wichtige Institutionen und Organisationen identifiziert werden, die unbedingt in ein solches Netzwerk einbezogen werden sollten: Wen gibt es vor Ort, der einen relevanten Beitrag leisten könnte? Wer könnte Interesse an einer Kooperation haben? Was könnte der jeweilige Beitrag sein? Wichtig ist, dass eine Entscheidung, welche Organisation unbedingt einbezogen werden sollte, keine grundsätzliche Entscheidung gegen eine andere Organisation darstellt, sondern dass vielmehr die „Modellierung“ des Netzwerks einen Prozess darstellt, der über den Zeitpunkt der formalen Konstitution hinausreicht. Dennoch sollte vor der Konstitution überlegt werden, wie groß ein Produktionsnetzwerk sein sollte, um (noch) steuerbar zu sein. Für die spätere Arbeit im Netzwerk braucht es nicht allein die Kenntnis der vorfindlichen Strukturen („Institutionenlandschaft“), sondern es bedarf darüber hinaus der Sensibilität für die unterschiedlichen Perspektiven, die die einzelnen Akteure haben, sowie für die fachspezifischen Sinnsysteme und „Fachsprachen". Die notwendige Heterogenität des Netzwerks bringt es nämlich mit sich, dass Akteure zusammenarbeiten werden, die sich auf ganz unterschiedliche Traditionen, gesetzliche Aufträge oder Ziele berufen (vgl. Abschnitt 4.4). Nachdem die potenziellen Netzwerkbeteiligten ausgewählt 
sind, gilt es, sie für die gemeinsame Sache zu gewinnen. Sind die zu gewinnenden Organisationen bekannt, geht es darum, dort auch die geeigneten Personen anzusprechen, die sich nicht nur für die Idee interessieren, sondern sie auch in der Organisation weiter verbreiten und für Akzeptanz sorgen. Hierfür gibt es den Begriff der „boundary spanner“ (Borkenhagen u. a. 2004: S. 23), d. h. der "Grenzgänger“, die in der Lage und bereit sind, über den Tellerrand der Organisationsinteressen hinauszublicken und später vielleicht auch die Organisation im Netzwerk zu vertreten. Bereits zu diesem frühen Zeitpunkt zeigt sich das Kernproblem institutioneller Netzwerke: Sie bestehen aus Personen, die im Netzwerk Organisationen mit eigenständigen Interessen repräsentieren. Als Mitglieder (häufig: Beschäftigte) dieser Organisationen müssen sich diese Personen gegenüber der Herkunftsorganisation loyal verhalten, gleichzeitig sollten sie im Interesse des gesamten Netzwerks handeln.

Um Organisationen für die Arbeit in einem Netzwerk zu gewinnen (d. h. im Minimum, dass sie bereit sind, Personen in Sitzungen des Netzwerks zu entsenden), muss transparent gemacht werden, was sie von ihrer Beteiligung haben. Hierzu gilt es zunächst, potenzielle Win-win-Situationen für die möglichen Netzwerkpartnerinnen und -partner auszuloten und abzuschätzen, was in welcher Zeit gemeinsam erarbeitet werden könnte. Diese Win-win-Situationen müssen dann den Beteiligten aber auch deutlich sein, d. h. als solche wahrgenommen werden (vgl. Bienzle u. a. 2007: S. 21).

\subsubsection{Allokation: Verteilung der Aufgaben in der Dienstleistungskette}

Für die interne Stabilisierung und für die Außendarstellung eines Netzwerks ist es wichtig, dass die Akteure gemeinsam „Produkte“ oder „Projekte" erarbeiten, die einen festeren Netzwerkzusammenhang schaffen und identitätsbildend wirken. Bei aller Skepsis gegenüber isolierten „Maßnahmen“ (im Sinne von „operativen Inseln“, vgl. Schubert 2008) darf nicht vergessen werden, dass erst ein konkreter Arbeitszusammenhang einen Rahmen für die Erfahrung von „Verlässlichkeit" der Kooperationsbeteiligten und damit die Tragfähigkeit der Netzwerke bildet. Wichtig ist es, den Doppelcharakter dieser „Produkte“ zu sehen: Sie haben ihren „Eigenwert" im Nutzen, den sie für die Zielgruppe stiften (z. B. einen „Leitfaden“ oder eine „Anlaufstelle“), gleichzeitig stellen sie den Kulminationspunkt für die Schaffung einer nachhaltigen Unterstützungsstruktur, d. h. den Aufbau und die Stabilisierung eines Produktionsnetzwerks, dar. Diese Produkte sollten „möglichst bereits in der Initiierungsphase eines Netzwerks zu 
wahrnehmbaren positiven Ergebnissen sowohl für das Netzwerk wie auch für die Netzwerkpartner (Wahrnehmen der Win-Win-Situation) führen“ und „das Know-How möglichst vieler Partner benötigen; die Stärken eines Partners sowie der jeweiligen Schnittstellen deutlich werden lassen." (Borkenhagen u. a. 2004: S. 46) In der gemeinsamen Arbeit wird persönliches Kennenlernen möglich und „Verlässlichkeit“ erfahrbar. In diesem Sinne ist ein „Produktionsnetzwerk“ ohne konkretes Produkt kaum vorstellbar. Umgekehrt sind zumindest die Produkte, die im sozialen Bereich auf komplexe Bedarfslagen zielen, ohne Netzwerk kaum zu realisieren.

Grundsätzlich besteht die konzeptionelle Alternative zur Realisierung eines solchen Produkts

- in der Netzwerkbildung (als konkreten Kulminationspunkt der Aktivitäten) oder

- in der Entwicklung von Produkten aus einem bereits bestehenden Netzwerkzusammenhang, der sich dann in der Produktentwicklung konkretisiert und stabilisiert.

Die Funktion der Produktentwicklung für die Netzwerkbildung besteht darin, dass ein konkretes gemeinsames Ziel erarbeitet und definiert wird, aus dem Aktivitäten abgeleitet werden, an denen tendenziell alle Akteure beteiligt sind. Diese gemeinsam erarbeiteten Produkte festigen den Zusammenhang der Akteure, die konkrete Zusammenarbeit wird erprobt, Kenntnisse über Schnittstellen werden erworben und Ansätze zu ihrer Überwindung können entwickelt werden. Das Netzwerk wächst über die Gestaltung des Prozesses: Ausgangspunkt eines an dieser Vorgehensweise orientierten Umsetzungsprozesses ist die Definition von im Netzwerk gemeinsam getragenen Zielen. Motivationsstiftend an dieser Arbeit ist, dass die Autonomie der Akteure nicht in Frage gestellt, sondern vielmehr eine gemeinsame Aufgabe spezifiziert wird.

\subsubsection{Regulation: Festlegung von Regeln und Strukturen}

Ein wichtiger Bestandteil dieser Funktion ist die Stabilisierung eines einmal gegründeten Netzwerks durch dessen „Regulation“ (vgl. Sydow/Möllering 2009: S. 189). Diese kann darin bestehen, Strukturen festzulegen, Regeln zu definieren oder Personen zu veranlassen, innerhalb des Netzwerks tätig zu werden. Ein weiteres wichtiges Element ist darüber hinaus die beständige Bekräftigung von „Verlässlichkeit“ als Steuerungsmodus. Erst die Sicherheit, zumindest mittelfristig nicht nur zu geben, sondern auch nehmen zu können, bewegt Akteure des Netzwerks dazu, ein Stück ihrer organisationa- 
len Autonomie in ein gemeinsames Vorhaben „einzubringen“, ohne die Autonomie jedoch ganz zur Disposition zu stellen und sich abhängig vom Netzwerk zu machen. Viele Autorinnen und Autoren sehen hierin ein Wesensmerkmal von Netzwerken, das sie von formalen Organisationen unterscheidet, die meist durch klare Weisungsverhältnisse gekennzeichnet sind. Gleichzeitig macht dieses Merkmal ihre Fragilität aus und stellt eine beständige Bedrohung der Stabilität von Netzwerkbeziehungen dar - wenn nämlich die Verlässlichkeit von einzelnen Akteuren in Frage gestellt und in der Folge durch das Überhandnehmen von Misstrauen unter Umständen tatsächlich fraglich wird: „Ein Netzwerk ist nur so lange in Betrieb, wie die Partner damit rechnen, dass institutionelle Win-Win-Situationen in einer für sie angemessenen Zeit und in einer angemessenen Art und Weise eintreten werden" (Borkenhagen u. a. 2004: S. 49)

Die Stabilität bildende Identität eines Netzwerks wird in einem ersten Schritt über die Formulierung gemeinsam geteilter Ziele hergestellt. Diese „tragen intensiv dazu bei, dass eine gemeinsame Wahrnehmung und eine ähnliche Bewertung der gegenwärtigen Situation wie auch der zukünftigen Aussichten der Zusammenarbeit entstehen und erhöhen damit die Kooperationsbereitschaft der Netzwerkakteure“ (Sydow 1999: S. 262). Die aus dem Projektmanagement bekannten Regeln der Zielformulierung sind zu beachten, damit Ziele auch handlungsleitend sind und bleiben: „Die Ziele müssen präzise formuliert sein. Eine ungefähre Verabredung auf vage Netzwerkziele führt zu Unzufriedenheit und selten zum Erfolg." (Borkenhagen et al. 2004: S. 36) Zielerreichung setzt einerseits die Verlässlichkeit der Netzwerkpartnerinnen und -partner voraus und bildet umgekehrt für diese eine wichtige Motivation zu verlässlichem Handeln. Verlässlichkeit wird von den Beteiligten über das Einhalten gemeinsam aufgestellter Regeln, die Ausführung übertragener Aufgaben, aber vor allem über die Erfahrung der Durchführung gemeinsamer Projekte signalisiert. „Regulation" bedeutet in diesem Zusammenhang zunächst die Aufgabe, zentrale Regeln zu erarbeiten, die mehr oder minder formalisiert sein können. Die Erarbeitung sollte möglichst frühzeitig geschehen und die Regeln sollten verbindlich, aber flexibel sein. Entscheidend ist, dass sie von allen Akteuren akzeptiert werden können und sich niemand übervorteilt vorkommt. Dies verlangt der Netzwerkkoordination höchste Neutralität und Fingerspitzengefühl ab: Sie ist „Anwalt des Netzwerks“ (ebd.: S. 47), soll alle Beteiligten einbinden, ihnen aber immer das Gefühl geben, ein freiwilliges Bündnis und keine Zwangsgemeinschaft eingegangen zu sein.

Regeln werden dadurch lebendig, dass sie in gemeinsamen Aktivitäten bestätigt werden, insofern ist die gemeinsame Arbeit an Produkten ein 
wichtiges „Bindemittel“ für Netzwerke. Netzwerkkoordination bedeutet somit auch die Planung und Strukturierung gemeinsamer Vorhaben und Netzwerkmanagement benötigt „Integrationskompetenz“ (ebd.: S. 52), z. B. die gemeinsame Strukturierung (und ggf. das Monitoring) von Abläufen. Der Verstetigung dienen aber auch Alltagsaufgaben wie die Dokumentation aller wichtigen Arbeitsschritte sowie die Führung und Verteilung von Protokollen.

\subsubsection{Evaluation: Auswertung und Bewertung der gemeinsamen Arbeit}

Eine wichtige Funktion für die Sicherung der Nachhaltigkeit von Netzwerkstrukturen besteht in der Beobachtung und Auswertung des gemeinsamen „Produktionsprozesses“ und seiner Resultate. Es hängt von der Art des Netzwerks, dem Grad an Verbindlichkeit und auch von den zur Verfügung stehenden Ressourcen ab, in welcher Form und mit welchem Grad an Formalisierung (Controlling, externe Evaluation) die Beobachtung und Bewertung erfolgt. Wesentlich ist es, alle Akteure aktiv einzubeziehen und gemeinsame Diskurse zu organisieren, um diese Funktion für die Stabilisierung und Verstetigung von Netzwerkbeziehungen zu nutzen.

\subsection{Ein Beispiel: Planungsprozesse am Standort Z}

\subsubsection{Vorbemerkung}

Das in den Abschnitten 4.4 bis 4.6 skizzierte Vorgehen bei der Bildung von Produktionsnetzwerken wurde den am Projekt beteiligten Kommunen vorgestellt. Sie konnten sich dafür entscheiden, die jeweiligen Prozesse der Netzwerkbildung (auch in modifizierter Form) daran auszurichten oder ein eigenständiges Procedere zu wählen. Die allermeisten Standorte entschieden sich für das hier dargestellte Verfahren.

Generell wurden die gemeinsamen Arbeitsprozesse protokolliert, so dass die Entwicklung an den einzelnen Standorten für die Leitung des Gesamtprojekts immer transparent war. Auf diese Weise wurde Material erstellt, das für die Auswertung des Projekts „Einwanderung gestalten“ von hohem Wert war und ist. Im Folgenden ist die Entwicklung an einem (anonymisierten) Standort wiedergegeben, da sowohl Vorgehensweise wie Protokolllage besonders gut geeignet scheinen, die praktische Umsetzung der oben wiedergegebenen Überlegungen an einem Beispiel zu illustrieren. 


\subsubsection{Standort Z - von Fällen zu Zielen ${ }^{34}$}

Am Standort $\mathrm{Z}$ existiert eine Lenkungsgruppe, welche das höchste Gremium auf Arbeits- und Entscheidungsebene darstellt. Während der Projektlaufzeit tagt sie anfänglich im viertel-, später im halbjährlichen Turnus. Als beschlussfähiges und entscheidendes Gremium besteht sie sowohl aus unterschiedlichen migrations- und arbeitsmarktpolitisch relevanten als auch aus Akteuren des Feldes Sozialer Hilfen: dem Fachbereich für Schulen, Soziales, Migration und Integration und dem dort angesiedelten Team „Beratung", der Arbeitsagentur, dem Jobcenter, einer Vertretung des Ehrenamtes, der Ausländerbehörde, der Volkshochschule, der Wirtschaftsförderung, dem Migrationsdienst sowie der Caritas und dem Kommunalen Integrationszentrum des Kreises Q. Teilnehmende sind Leitungskräfte der genannten Organisationen. Der Lenkungsgruppe kommt die Aufgabe zu, Mitglieder für die jeweiligen Arbeits- und Projektgruppen zu benennen. Außerdem legitimiert die Lenkungsgruppe die Projektkoordinatorinnen, damit diese mit der operativen Ebene der verschiedenen beteiligten Institutionen arbeitsfähig werden können.

Am Standort $Z$ resultieren im Projektverlauf aus der Arbeit einer vorgeschalteten Arbeitsgruppe - an welcher relevante und für das Feld bedeutende Akteure teilnehmen - zwei Arbeitsgruppen. Der Fokus der ersten Arbeitsgruppe liegt auf dem Thema „Arbeit“, der zweiten auf dem Thema „Sprache“; die zwei „großen Bereiche“. Diese beiden Arbeitsgruppen starten $\mathrm{zu}$ je unterschiedlichen Zeitpunkten und sind mit unterschiedlichen Akteuren besetzt. In der vorgeschalteten Arbeitsgruppe wird zu Beginn mit Fällen gearbeitet, aus welchen sich die oben genannten Schwerpunkte "Sprache“ und „Arbeit“ herauskristallisierten. Der Prozess in Z. gestaltet sich so, dass die Arbeitsgruppe mittels Fallrekonstruktionen "generative Themen" herausarbeitete, nach „Hemmnissen“ schaute und Bearbeitungsvorschläge entwickelte. Diese Vorschläge wiederum wurden dann in die Lenkungsgruppe gegeben, dort erneut verhandelt und ggf. nochmalig zur Überarbeitung zurückgespielt. Den Teilnehmenden der Arbeitsgruppe kommt also die Aufgabe zu, im Rahmen von Planungskonferenzen Prozessbetrachtungen zu ermöglichen. Resultierend hieraus wird in Z. für die beiden „großen Bereiche“ jeweils ein dreigliedriges Zielsystem (Grundsatz-

34 Dieser Abschnitt wurde von David Hübner verfasst. Wenn im Folgenden in direkter oder indirekter Weise zitiert wird, bezieht sich der Autor auf durch Mitarbeitende des Standorts verfasste und zur Verfügung gestellte Protokolle einzelner Arbeits-, Lenkungs- und Projektgruppensitzungen. 
ziele, Rahmenziele, Ergebnisziele) entwickelt. Die relevanten Akteure wurden vom Projektteam im Vorhinein gebeten, aus ihrer Arbeit „typische Fälle“ einzubringen, welche dann für die entsprechenden Sitzungen durch die Projektkoordinatorinnen und -koordinatoren ausführlich und systematisiert vor- und aufbereitet wurden. Das Vorgehen der Arbeit an „typischen Fällen“ verfolgt das Ziel der „Herausarbeitung struktureller Elemente“ und stellt nicht den Versuch einer „Falllösung“ dar. Die Sitzungen werden dazu genutzt, sich entlang der Fälle unter den unterschiedlichen Akteuren auszutauschen und den je spezifischen Blick auf, den Fall' hinsichtlich der Frage nach „Unterschiedlichkeiten“ und „Parallelen“ auszuloten.

Im Folgenden soll ausschnitthaft die Genese und darin eingelagerte Aushandlungen veranschaulicht werden.

Die Fälle

Die übergeordnete und vorgeschaltete Arbeitsgruppe beschäftigte sich in den ersten beiden zeitlich dicht aufeinander folgenden Sitzungen mit drei „typischen Fällen“. Im Anschluss an die unten folgende Darstellung des dritten Falls wird die daran anschließende Diskussion inklusive Fallrekonstruktion exemplarisch ausgeführt.

Der erste Fall 35 :

„Herr A. ist 33 Jahre alt. Er ist verheiratet und hat einen 4 jährigen Sohn. Seine Familie lebt zurzeit im Iran. Auch Herr A. ist aus dem Iran nach Deutschland geflohen. Er wurde im Februar 2015 der Stadt Z. als Asylbewerber zugewiesen.

Herr A. arbeitet seit Eröffnung der Kolping-Fahrradwerkstatt bis jetzt ehrenamtlich, zeitweise auch als gemeinnütziger Arbeiter $(0,80 €)$ mit. Er hatte auch im Iran in einer Fahrrad-Fertigung gearbeitet. Durch freundschaftliche Kontakte zu den Kolping-Mitgliedern hat er viel ehrenamtliche Unterstützung und Familienanschluss.

Ab August 2015 bis Juli 2016 besuchte er die Abendrealschule. Herr A. wollte Deutsch lernen, aber sein Hauptanliegen war es eine Arbeit zu finden. Mit Unterstützung der Kolpingwerkstatt und der zuständigen Fachstelle hat er sich bei allen umliegenden Fahrrad-Firmen um ein Praktikum bzw. um eine Arbeitsstelle beworben. Leider bekam er keine positive Rückmeldung.

Danach nahm Herr A. Kontakt zum Integration Point der Agentur für Arbeit auf. Über die Mitarbeitenden konnte er an einer Maßnahme bei der Kreishand-

35 Inwieweit zur Erfüllung anderer Netzwerkfunktionen "Netzwerkmanagement“ notwendig ist, ist eine eher empirisch zu beantwortende Frage. Auch muss geklärt werden, welche Funktionen des Netzwerkmanagements für unterschiedliche Konstellationen notwendig erfüllt werden müssen. 
werkerschaft teilnehmen (Dauer ca. 2 Monate). Ab Oktober 2016 arbeitet Herr A. bei einer Zeitarbeitsfirma. Die ständig wechselnden Einsatzorte und die damit verbundene Organisation der Fahrten zur Arbeit belasteten ihn. Im Dezember 2016 bekam er die Flüchtlingseigenschaft zuerkannt, im Januar 2017 die Wohnsitzauflage nach $\$ 12$ a für die Stadt Z. Seine Familie hatte inzwischen einen Antrag auf ein Visum zur Familienzusammenführung gestellt. Wann das Visum ausgestellt wird ist noch unklar. Den Flug nach Deutschland muss die Familie selbst bezahlen, so dass Herr A. froh war Arbeit zu haben. Nach einigen Wochen verlor Herr A. seinen Arbeitsplatz, da der Aufwand die verschiedenen Arbeitseinsatzorte pünktlich zu erreichen, seine finanziellen und sonstigen Mittel überforderten.

Die Sozialarbeiterin der zuständigen Fachstelle vermittelte einen Termin mit dem Jobcenter, da Herr A. einen SGB II-Antrag stellen musste, um seinen Lebensunterhalt zu sichern. In dem Beratungsgespräch mit der Arbeitsvermittlung wurde er verpflichtet einen Integrationskurs zu belegen und erst nachfolgend evtl. Qualifizierungen, bzw. eine versicherungspflichtige Tätigkeit aufzunehmen. Zurzeit ist Herr A. auf der Warteliste für einen Integrationskurs, nachdem er längere Zeit bereits auf den Einstufungstest warten musste. Diese Wartezeiten sind auch durch fehlende Unterlagen bei Abgabe des Berechtigungsscheins zum Integrationskurs beim Sprachkursträger verursacht. Herr A. bemüht sich weiterhin um einen Arbeitsplatz, um Geld für den Flug seiner Familie verdienen zu können“.

Der zweite Fall:

„Das Ehepaar M. (Mann 40, Frau 35) hat fünf Kinder. Das älteste ist 13 und das jüngste 1 Jahr alt. Vier der Kinder gehen zur Schule. Für das jüngste ist noch keine Kinderbetreuung gesichert. Herr M. hat einen Schulabschluss in Syrien gemacht (vergleichbar mit dem deutschen Abitur), aber keine weiteren Qualifikationen. Er hat in Syrien diverse Berufe ausgeübt: Friseur, Taxifahrer, und zum Schluss besaß er einen eigenen Lebensmittelladen. Seine Schulurkunde wurde durch den Krieg vernichtet/ist verloren gegangen. Bevor Herr M. als Flüchtling anerkannt wurde und er noch Asylleistungen bezog, wurde er von der Arbeitsagentur (Integration Point) in eine Maßnahme zur beruflichen Vorbereitung vermittelt. An der nahm er allerdings unregelmäßig teil. In Absprache mit der Arbeitsagentur hat der Träger Herrn M. abgemahnt und ein Gespräch zur Ursachenklärung geführt. Jedoch fehlte er weiterhin ohne triftigen Grund oder einer Arbeitsunfähigkeitsbescheinigung. Die Fehlzeiten hatten letztendlich den Ausschluss aus der Maßnahme zur Folge. Frau M. hat keinen Schulabschluss und hat keinerlei Berufserfahrung. Vermutlich ist sie in der lateinischen Schrift nicht oder schlecht alphabetisiert. Sie befindet sich derzeit in der Elternzeit und möchte diese voll ausschöpfen. Aus Sicht der Arbeitsvermittlung/rechtlich gesehen ist Herr M. zu einer Teilnahme am Integrationskurs verpflichtet. Er gibt allerdings an, dass er die Kinder jeden Tag zur Schule begleiten und sie nach dem Unterricht abholen muss. Dies würde sehr viel Zeit in Anspruch nehmen, da sie mit den öffentlichen Verkehrsmitteln zu unterschiedlichen Schulen fahren müssen. Frau M. könne diese Aufgabe nicht übernehmen, da dies mit dem jüngsten Kind 
nicht machbar wäre. Herr M. wird mehrfach darauf hingewiesen, dass einer der beiden Elternteile am Integrationskurs teilnehmen muss, damit eine Arbeitsaufnahme im Anschluss erfolgen kann. Daraufhin meldet er sich beim Träger an, meldet sich jedoch einen Tag vor Kursbeginn, ohne Rücksprache mit dem Arbeitsvermittler, wieder ab. Dies geschieht nach einer sechsmonatigen Wartezeit. Herr M. kommt somit erneut auf die Warteliste und muss nochmals mindestens sechs weitere Monate auf den neuen Kursstart warten“.

Der dritte Fall:

„Herr R. ist ein junger Mann (23) aus Syrien, der eine sozialversicherungspflichtige Tätigkeit hatte, der Arbeitsvertrag jedoch aufgrund des Sprachmangels nicht verlängert wurde. Das Jobcenter verpflichtet ihn zur Teilnahme am Integrationskurs. Er wird mehrfach aufgefordert sich für einen Integrationskurs anzumelden. Dieser Aufforderung kommt er nicht nach.

Die Konsequenz daraus besteht in Sanktion, weshalb er sich daraufhin dann doch anmeldet.

Die Wartezeit beträgt 1 Jahr. Der Grund: Herr R. erhält einen Berechtigungsschein vom Jobcenter. Dieser Berechtigungsschein ist jedoch ungültig, da sich Herr R. bei einem anderen Träger an seinem alten Wohnsitz für einen Integrationskurs angemeldet hatte. Die Anmeldung erfolgte mit einem Berechtigungsschein von der Ausländerbehörde.

Die Konsequenz ist nun, dass der alte Berechtigungsschein vom alten Träger zurückgefordert werden und beim neuen eingereicht werden muss. Das Jobcenter wird vom BAMF aufgefordert, den ausgestellten Berechtigungsschein zurückzuziehen. Der bürokratische Aufwand hat zur Verzögerung der Anmeldung geführt. Herr R. erhält endlich einen Brief zum Kursbeginn. Kurz darauf legt er einen Arbeitsvertrag (sozialversicherungspflichtig; Teilzeit) vor und meldet sich vom Sprachkurs ab.

Die Arbeit wird ihm nach 3 Monaten gekündigt. Der Grund: die Sprachbarriere. Herr R. wird vom Jobcenter erneut aufgefordert sich für einen Integrationskurs anzumelden.

Die Anmeldung erfolgt, Herr R. nimmt am Kurs teil. Herr R.' Verhalten im Sprachkurs ist unangebracht, weshalb ihm vom Träger ein Hausverbot erteilt und sein Kurs abgebrochen wird. Herr R. wird daraufhin vom Jobcenter verpflichtet, sich bei einem anderen Träger anzumelden.

Dem zuständigen Arbeitsvermittler fallen bestimmte Verhaltensmuster auf, was die Vermutung zulässt, dass Herr R. psychisch angeschlagen ist. Ihm werden die Kontaktdaten des sozialpsychiatrischen Dienstes und der psychosozialen Flüchtlingshilfe mitgegeben. Allerdings sieht Herr R. nicht ein, dass eine Beratung vorteilhaft sein könnte. Zudem deutet er an, dass er nicht in der Lage wäre sich mit den Angestellten vernünftig zu unterhalten“. 
Auf die Schilderung der jeweiligen Fallskizze erfolgt in einem zweiten Schritt eine Diskussion der unterschiedlichen Perspektiven entlang bestimmter Fragestellungen: Dies ist hier exemplarisch für den dritten Fall dargestellt.

- Was ist der "Fall" (gesetzlicher/institutioneller Auftrag)?

\begin{tabular}{|c|c|}
\hline $\begin{array}{l}\text { Behörde/ } \\
\text { Institution }\end{array}$ & Informationen $^{36}$ \\
\hline $\begin{array}{l}\text { Bundesagentur für } \\
\text { Arbeit }^{37}\end{array}$ & - Weiterleitung zum I-Kurs \\
\hline $\begin{array}{l}\text { Fachbereich für } \\
\text { Migration und } \\
\text { Integration }\end{array}$ & $\begin{array}{l}\text { - } \text { Berechtigung vom alten Wohnsitz ordern oder } \\
\text { - } \text { Ausstellung einer Berechtigung für den I-Kurs }\end{array}$ \\
\hline Jobcenter (aktiv) & $\begin{array}{ll}\text { - } & \text { Sprachaufbau } \\
\text { - } & \text { Eingliederungsvereinbarung erst nach Starttermin } \\
& \text { des Sprachkurses, muss konkret sein (Unterrichts- } \\
& \text { zeiten, Träger, ...) }\end{array}$ \\
\hline Caritasverband & - Beratungsangebot \\
\hline $\begin{array}{l}\text { Ausländer- } \\
\text { behörde }\end{array}$ & $\begin{array}{l}\text { - } \text { Ruhezeit wegen bestehender Aufenthaltserlaubnis } \\
\text { - } \text { Blick auf Sprachkursabschluss wegen Aufenthalt }\end{array}$ \\
\hline $\begin{array}{l}\text { Jobcenter } \\
\text { (Leistung) }\end{array}$ & - Sanktionen wegen fehlender Mitwirkung \\
\hline Volkshochschule & - I-Kurs \\
\hline
\end{tabular}

- Was sind aus Sicht der Akteure zentrale Themen?

\begin{tabular}{|l|l|}
\hline Volkshochschule & $\begin{array}{l}- \\
\text { Zugang zum Berechtigungsschein - I-Kurs? } \\
\text { (ggf. "alte“ Verpflichtung vorhanden, die erst rück- } \\
\text { gängig gemacht werden muss, damit "neuere“ Ver- } \\
\text { pflichtung greift }\end{array}$ \\
\hline $\begin{array}{l}\text { Kommunales } \\
\text { Integrations- } \\
\text { zentrum }\end{array}$ & $-\begin{array}{l}\text { Vernetzung von Projekten mit betreffenden The- } \\
\text { men } \rightarrow \text { Sprache }\end{array}$ \\
\hline
\end{tabular}

36 Die hier angeführten stichwortartigen Informationen/Positionen finden sich in dieser Form im Originalton im Protokoll AG II des Standorts Z. wieder.

37 Wegen der Nachvollziehbarkeit werden die im Protokoll ursprünglich genutzten Abkürzungen der Organisationen/Institutionen hier ausgeschrieben. 


\begin{tabular}{|l|l|}
\hline Ehrenamt & $\begin{array}{l}- \text { Auffälligkeit zieht sich durch das Verhalten } \\
-\end{array}$ \\
\hline $\begin{array}{l}\text { Fehlender Informationsaustausch von Ehrenamt } \\
\text { und Ämtern über „Fehlverhalten“, „Auffälligkei- } \\
\text { ten“ }\end{array}$ \\
\hline $\begin{array}{l}\text { Kommunales } \\
\text { Integrationszentrum }\end{array}$ & $\begin{array}{l}\text { Sensibilisierung und Begleitung von Ehrenamt bei } \\
\text { auffälligem Verhalten }\end{array}$ \\
\hline Jobcenter (aktiv) & $\begin{array}{l}\text { Einsichtsfähigkeit für "Therapie“ muss vom Betrof- } \\
\text { fenen vorhanden sein }\end{array}$ \\
\hline
\end{tabular}

- Welches Ziel verfolgen die jeweiligen Akteure?

Hier wird kollektiv geäußert, dass es im ersten Schritt um einen Sprachkurs, im zweiten Schritt um die Feststellung der Leistungsfähigkeit gehen muss und dann weitere Schritte eingeleitet werden müssen.

- Welche Interventionsstrategien verfolgen die einzelnen Akteure?

\begin{tabular}{|l|l|}
\hline $\begin{array}{l}\text { Fachbereich für } \\
\text { Migration und } \\
\text { Integration }\end{array}$ & - Beratungsgespräche und Begleitung anbieten \\
\hline Jobcenter (aktiv) & $\begin{array}{l}\text { Vermittlung Gesundheitsamt/psychologischer } \\
\text { Dienst/Gutachten wird ggf. nicht mit der bzw. } \\
\text { dem Untersuchten besprochen. Sie bzw. er erhält } \\
\text { nicht das Gutachten, nur eine Mitteilung über die } \\
\text { Arbeitsfähigkeit }\end{array}$ \\
\hline Caritasverband & - Vernetzung/Andocken an Freizeitgruppen/Vereine \\
\hline Ehrenamt & - Verweigerung mangels Einsicht, Gesichtsverlust \\
\hline
\end{tabular}

- Welche Wirkungen haben diese Interventionen?

\begin{tabular}{|l|l|}
\hline Ehrenamt & - Verweigerung mangels Einsicht, Gesichtsverlust \\
\hline $\begin{array}{l}\text { Bundesagentur für } \\
\text { Arbeit }\end{array}$ & $\begin{array}{l}\text { Wenn Zuständigkeit gegeben -> Empfehlung ohne } \\
\text { Konsequenzen - evtl. Infoübermittlung an Fach- } \\
\text { stelle, dass er nicht zur Untersuchung erschienen } \\
\text { ist. Im größten Zweifel Abmeldung und Übergabe } \\
\text { an Sozialamt. }\end{array}$ \\
\hline $\begin{array}{l}\text { Jobcenter } \\
\text { (Leistung) }\end{array}$ & $\begin{array}{l}\text { Mögliche Sanktionen nach Bewilligungszeitraum, } \\
\text { wenn er die ärztliche Untersuchung verweigert. } \\
\text { Pflicht zur Mitarbeit. }\end{array}$ \\
\hline
\end{tabular}


Die teilnehmenden Akteure sind in die Beantwortung dieser Fragen unterschiedlich stark involviert, formulieren - wie deutlich wird nicht zu allen Fragen einen Vorschlag oder antworteten mitunter auch als Kollektiv.

Im dritten Schritt wurde durch die partizipierenden Akteure versucht, die Nutzerperspektive einzunehmen. Dies vollzog sich entlang der folgenden Fragen:

- Wie sieht der Nutzer die Situation?

\begin{tabular}{|c|c|}
\hline $\begin{array}{l}\text { Behörde/ } \\
\text { Institution }\end{array}$ & Informationen \\
\hline $\begin{array}{l}\text { VHS, Jobcenter } \\
\text { (aktiv) }\end{array}$ & $\begin{array}{l}\text { - Geld verdienen, er sieht sich als erwachsender } \\
\text { Mensch, der arbeiten möchte, "Arbeiten für die } \\
\text { Grundbedürfnisse, keine Selbstverwirklichung“ }\end{array}$ \\
\hline $\begin{array}{l}\text { Jobcenter (aktiv), } \\
\text { Caritasverband }\end{array}$ & - Seine Motivation ist Arbeit und nicht Sprache \\
\hline $\begin{array}{l}\text { Bundesagentur für } \\
\text { Arbeit }\end{array}$ & $\begin{array}{l}\text { Ständige Anforderungen seitens der Akteure (BA, } \\
\text { Jobcenter, Beratung), auch wenn er kein Ziel hat }\end{array}$ \\
\hline Ehrenamt & $\begin{array}{l}\text { - Fremdbestimmung; Abweichung der gestellten } \\
\text { Anforderungen von den eigenen Wünschen wie } \\
\text { Familie nachholen etc., unter Druck, er will gar } \\
\text { nichts mehr }\end{array}$ \\
\hline Volkshochschule & $\begin{array}{l}\text { - Immer wieder sein(en) Leben/Lebensverlauf offen- } \\
\text { legen }\end{array}$ \\
\hline Jobcenter (aktiv) & 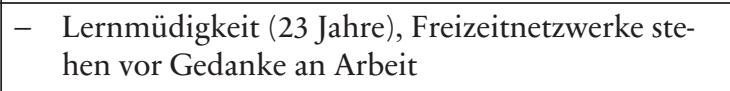 \\
\hline $\begin{array}{l}\text { Kommunales } \\
\text { Integrations- } \\
\text { zentrum }\end{array}$ & $\begin{array}{l}\text { - Freizeitorientiert, legt Arbeitsvertrag vor, wenn } \\
\text { Sprachkurs beginnt, geht in Teilzeit }\end{array}$ \\
\hline
\end{tabular}

- Wie sieht sich der Nutzer im Verhältnis zu weiteren Personen? (Familienmitgliedern)?

- Welche Erwartung hat der Nutzer an Angebote der Akteure?

- Was ist die Motivation des Nutzers für sein Handeln?

- Welche Handlungsoptionen hat der Nutzer?

- Was hat der Nutzer bereits unternommen?

- Welche Strategien werden sichtbar?

Die Beantwortung der anderen sechs Fragestellungen lässt keine unterschiedlichen Positionen erkennen, weshalb auf eine vertiefende Darstel- 
lung verzichtet wird. Zusammenfassend kann jedoch gesagt werden, dass die Informationsspalte die Themen Resignation, Überforderung, Unverständnis und Überforderung enthält.

Schritt vier am Standort $Z$ sieht nun die Fallrekonstruktion vor, in dessen „Zentrum der Versucht [steht], die Fallkonstellation als Wirkungsgefüge zu rekonstruieren. Ziel ist es zu sehen, wie die verschiedenen Aktivitäten der Beteiligten ineinander greifen, sich gegenseitig verstärken, bei vermehrten gegenseitigem Wissen verstärken könnten oder neutralisieren. Im Blickfeld ist nicht die Einzelfalllösung, sondern die Betrachtung der Schnittstellen, an denen die Stellschrauben ggf. etwas geändert werden sollten" (Protokoll AG II, Standort Z).

\begin{tabular}{|c|c|}
\hline \multicolumn{2}{|l|}{ Gemeinsame Fallrekonstruktion } \\
\hline $\begin{array}{l}\text { Wie sieht das "Wirkungsgefüge" im } \\
\text { Fall konkret aus? }\end{array}$ & $\begin{array}{ll}- & \text { I - Kurs Verpflichtung: Wer kümmert sich um } \\
& \text { Beibringung "alter“ Berechtigungsscheine? } \\
- & \text { Jobcenter (Aktiv) oder Bundesagentur für Arbeit } \\
\text { möchten Sprachkurs } \rightarrow \text { lange Wartezeit (im Fall } \\
\left.1 \text { ein Jahr }{ }^{38}\right) \rightarrow \text { Zusteuerung; Gründe, ggf. nicht } \\
\text { erschienen, Abmeldung, wieder auftauchen, } \\
\text { wollen zu keinem anderen Sprachkursträger als } \\
\text { VHS } \\
-\quad \text { Jobcenter (aktiv) und Sprachkursträger } \\
- & \text { Fehlende Info über Anmeldung, Teilnahme und } \\
& \text { Fehlzeiten, Jobcenter (aktiv) kann erst nach Wo- } \\
\text { chen reagieren } & \\
- & \text { Jobcenter (Leistung) und Jobcenter (aktiv): } \\
\text { Sanktionen nach fehlender Mitwirkung }\end{array}$ \\
\hline \multicolumn{2}{|l|}{$\begin{array}{l}\text { Veränderung von Stellschrauben } \\
\text { durch Einbeziehung der } \\
\text { Nutzerperspektive? }\end{array}$} \\
\hline $\begin{array}{l}\text { Sind Interventionen dadurch neu/ } \\
\text { anders zu denken? }\end{array}$ & $\begin{array}{ll}\text { - } & \text { Jobcenter Zugang zum BAMF - Sprachkurssys- } \\
\text { tem online zur Einsicht und Zusteuerung; auf- } \\
\text { grund Kennziffer ist ersichtlich, ob Einstufungs- } \\
\text { kurs gemacht wurde und Anmeldung zum Kurs } \\
\text { erfolgte } \\
\text { - } \\
\text { Direkte Meldung zwischen VHS - Jobcenter (ak- } \\
\text { tiv) - BA; Keine Info bzw. sehr späte Info über } \\
\text { Maßnahmenteilnahme bei Jobcenter (aktiv) }\end{array}$ \\
\hline
\end{tabular}

38 Mit Fall 1 ist der erste der oben dargestellten drei „typischen Fälle“ gemeint. 


\begin{tabular}{|c|c|}
\hline \multicolumn{2}{|l|}{ Gemeinsame Fallrekonstruktion } \\
\hline $\begin{array}{l}\text { Wie sieht die aktuelle Situation aus? } \\
\text { Ist eine Falldynamik zu erkennen? }\end{array}$ & $\begin{array}{l}\text { Erläuterungsbeispiel zu Punkt 4: Wären die Inter- } \\
\text { ventionen anders, bspw. Zugang von Jobcenter auf } \\
\text { BAMF-System, dann würden bei der VHS nicht } \\
\text { mehr so viele mit ungültiger Bescheinigung auflau- } \\
\text { fen. } \\
-\quad \text { Ja, Verkürzung von Zeiten (Wartezeit "Berechti- } \\
\quad \text { gungsschein“) } \\
-\quad \text { Verkürzung der Wartezeiten Sprachkurs } \\
-\quad \text { Verkürzung der Interventionsmöglichkeiten } \\
-\quad \text { Schnellere Reaktionszeit - Schnellere Steue- } \\
\text { - } \quad \text { Vermittlung Lobby - Beratungsstelle }\end{array}$ \\
\hline $\begin{array}{l}\text { Was sind in diesem Fall die } \\
\text { zentralen fallübergreifenden } \\
\text { Themen? } \\
\text { Betrifft die Fälle } 1 \text { bis } 3\end{array}$ & $\begin{array}{ll}- & \text { Zielkonflikte, bspw.Jobcenter (aktiv) } \rightarrow \text { I-Kurs } \\
& \text { Nutzer } \rightarrow \text { Arbeit } \\
- & \text { Sprache } \\
& \rightarrow \text { wenig Kommunikation zwischen den Akteu- } \\
& \text { ren } \\
- & \text { Sprache, Ziel I - Kurs } \\
- & \text { Berechtigungsscheine (dreifache Ausstellung } \\
\text { möglich) }\end{array}$ \\
\hline $\begin{array}{l}\text { Weitere zusätzliche } \\
\text { fallübergreifende Themenfelder } \\
\text { Betrifft die Fälle } 1 \text { bis } 3\end{array}$ & $\begin{array}{ll}- & \text { Sucht } \\
- & \text { Gesundheit } \\
- & \text { Schulden } \\
- & \text { Sprachkurse für Frauen (mit Kindern) }\end{array}$ \\
\hline
\end{tabular}

In der darauffolgenden Sitzung der Lenkungsgruppe werden die Ergebnisse der drei Fallrekonstruktionen, welche mittels der Planungskonferenzen in den Arbeitsgruppen stattfinden, den vertretenen Teilnehmerinnen und Teilnehmer der Lenkungsgruppe durch die Projektleitung vorgestellt. Es wird hier hervorgehoben, dass mittels der Rekonstruktionen der Fälle, fallübergreifende Themen aufgetaucht sind, welche wie folgt zusammengefasst werden können: Ausreichende Kursangebote, funktionierende $\mathrm{Zu}$ gangssteuerung, Informationsfluss und -Weg, Wartezeiten bis der Integrationskurs beginnt, Trägerabsprachen und die Ausgabe des Berechtigungsscheins.

Unter den verschiedenen Ergebnissen wird dann der in der Fallrekonstruktion herausgearbeitete Berechtigungsschein für Zugewanderte zum Integrationskurs ausgewählt. Dieser stelle einerseits eine Grundvoraussetzung für die Teilnahme am Integrationskurs dar und habe außerdem verpflichtenden Charakter. Diesen Berechtigungsschein können drei Institutionen ausstellen: die Asylleistungsgewährungsstelle, die Ausländerbehör- 
de und das Jobcenter. Bei der Asylleistungsgewährungsstelle kann es online innerhalb von zwei Minuten erledigt werden (berechtigt ja oder nein), woraufhin eine sogenannte Personenkennziffer ausgestellt wird. Der Zugang ist hier lediglich Geflüchteten gestattet, die sich bereits im Asylverfahren befinden. Bei der Ausländerbehörde ist die Zielgruppe eine andere. Auch hier laufe es online, allerdings für Anerkannte, im Rahmen von Familiennachzug und Personen die aufgrund einer Wohnsitzzuweisung nach $\$ 12$ (a) nach Z. kommen. Das Jobcenter wiederum kann online keinen Berechtigungsschein ausstellen, mit der Begründung, dass die Zielgruppe Anerkannte im SGB II-Bezug seien. Der Prozess laufe hier so, dass die Mitarbeitenden die Nutzerinnen und Nutzer fragen, ob bereits ein solcher Berechtigungsschein ausgestellt wurde, worauf es unterschiedliche Antwortmöglichkeiten gebe. Allesamt führen zu massiven zeitlichen Verzögerungen und dauern wesentlich länger als eine Online-Abfrage. Abschließend wird festgehalten, dass das Online-Verfahren das schnellste und auch sicherste Verfahren darstelle, um den Berechtigungsschein zu erhalten. Außerdem entfallen etwaige Wartezeiten (Kostenbefreiungsantrag, Erteilung Personenkennziffer), die teilweise bei bis zu acht Wochen liegen können.

Im Anschluss an die Darstellung der drei involvierten Institutionen folgt eine Rückfragerunde/Diskussion. Im Anschluss wird durch die Projektkoordinatorinnen und -koordinatoren darum gebeten, über zwei Beschlüsse abzustimmen:

- Die Lenkungsgruppe wendet sich an das kreisverantwortliche Jobcenter in Q, um der Regionalstelle in Z "zeitnah" einen Online-Zugang für die Ausstellung des Berechtigungsscheins im System des BAMF einzurichten.

- Das Jobcenter in Z und die Ausländerbehörde treffen die Vereinbarung, dass der Berechtigungsschein im Rahmen des SGB II-Bezugs nur durch das Jobcenter ausgestellt wird. Das Jobcenter (Arbeitsvermittlung) trägt die Pflicht, die Ausländerbehörde über die Ausstellung des Berechtigungsscheins via E-Mail zu informieren.

Anschließend bringt eine Teilnehmerin die Perspektive der Zugewanderten ins Spiel, da die zur Disposition stehende Beschlusslage nicht nutzerfreundlich sei. Es findet hier ein Rückkoppeln der Ergebnisse der Fallrekonstruktionen innerhalb der Arbeitsgruppe statt. Diese Ergebnisse hätten gezeigt, dass es gewinnbringender sei, wenn die Nutzerinnen und Nutzer sich "nicht dem System unterordnen" müssten, "sondern das System optimiert werde". Außerdem hebt sie auf das BAMF ab. Diese "Schnittstelle" sei im Rahmen des Projekts EWG "noch schwerer zu erreichen" als weitere "Dinge" auf regionaler Ebene. Eine weitere Position wird vom Jobcenter 
vertreten: Dies sieht zwar auf Regionalebene die Vorteile der Online-Anbindung, ist jedoch zentral von Q ausgeführt, wo gegenwärtig die OnlineAnbindung nicht stattfindet. Deshalb bleibe das Ergebnis der Beschlussvorlage - welche in dieser Sitzung vorgestellt wird - offen und als Auftrag verstanden mit $\mathrm{Q}$ zu kommunizieren. Der Beschluss wird dann konsensual angenommen.

Zielsystem

Resultierend aus der Arbeit mit Fällen in der Arbeitsgruppe und der Rückkopplungsschleife inklusive Beschlussfassungen durch die Lenkungsgruppe, wurden im weiteren Verlauf in den daraus entstehenden Arbeitsgruppen („Arbeit“ und „Sprache“) Zielsysteme entwickelt. Im Folgenden wird exemplarisch die Genese des Zielsystems für die „Arbeitsgruppe Sprache“ vorgestellt.

Das Vorgehen in Z. sieht so aus, „dass die LG (Lenkungsgruppe, Anm. d. Verf.) das GZ (Grundsatzziel, Anm. d. Verf.) festlegt und die operative Ebene beauftragt, ein Zielsystem für den Nutzer zu erarbeiten. Anschließend erfolge eine Rückkoppelung des Zielsystems an die LG. Die Ziele werden auf dieser Ebene dann bestätigt, entschieden oder auch abgelehnt" (Protokoll AG Sprache, II). Die Lenkungsgruppe hat zwei Grundsatzziele beschlossen. Aufgabe der Projektgruppenakteure sei es ein Zielsystem zu entwickeln, welches Rahmen- und Ergebnisziele sowie daraus resultierende konkrete Aktivitäten beinhaltet. Dieses differenzierte Vorgehen ermögliche es einerseits „(...) Stolpersteine“ zu identifizieren und die Sicht auf die Nutzerperspektive zu berücksichtigen, da diese „erforderlich ist, um für dessen Situation etwas zu erreichen" (Protokoll AG Sprache, II). Das konkrete Vorgehen in Z gestaltete sich so, dass durch die Projektkoordinatorinnen und -koordinatoren im Vorfeld Rahmen- und Ergebnisziele formuliert wurden, welche nun in der Arbeitsgruppe eine vertiefende Bearbeitung erfahren sollen. Mittels eines Flipchart wird den Akteuren die Möglichkeit offeriert, zwischen drei potenziellen Optionen ihre Meinung zu den jeweiligen Zielformulierungen zu äußern; „grün“ steht für einverstanden, „blau“ für Klärungsbedarf und „rot“ für Einspruch. Nach einer halbstündigen Bearbeitungszeit wird ein Rahmenziel exponiert behandelt, da dieses wenig Einverständnis auf Seiten der Akteure hervorgerufen hat. Es lautet: „Berechtigte Nutzer sind motiviert und in die Lage versetzt Integrationskurse zu besuchen“. Die Projektkoordinatorin begründet die Formulierung des Rahmenziels damit, dass „sich in den bisherigen Sitzungen der Arbeitsgruppe zeigte, dass das Nutzerverhalten in den Blick genommen werden muss." (Protokoll AG Sprache, II) Auf Nachfrage, weshalb dieses Rahmenziel auf solch geringe Zustimmung stoße, wird von der Ar- 
beitsgruppe formuliert, dass dieses Ziel als „schwierig“ erachtet werde. Insofern sei grün nicht die richtige Entscheidung, gleichwohl Konsens darüber existiert, dass das Rahmenziel „so bestehen bleiben und abgearbeitet werden" soll.

Das erarbeitete Zielsystem der „Projektgruppe Sprache“ für das Grundsatzziel 1 sieht wie folgt aus:

\begin{tabular}{|l|l|}
\hline Ziele & Zieldefinition \\
\hline Grundsatzziel & $\begin{array}{l}\text { Jeder berechtigte Zuwanderer belegt innerhalb von } \\
\text { 8 Wochen einen bedarfsgerechten Integrationskurs }\end{array}$ \\
\hline Rahmenziel 1 & $\begin{array}{l}\text { Innerhalb von drei Wochen hat jeder anspruchsbe- } \\
\text { rechtigte Zuwanderer einen Berechtigungsschein } \\
\text { (BS) für einen Integrationskurs }\end{array}$ \\
\hline Ergebnisziel 1.1 & $\begin{array}{l}\text { Asylbewerber mit guter Bleibeperspektive im Asyl- } \\
\text { verfahren bekommen bei Unterbringung den BS }\end{array}$ \\
\hline Ergebnisziel 1.2 & Eine Doppelausstellung wird vermieden \\
\hline Ergebnisziel 1.3 & Der schnellste Weg wird die Regel \\
\hline Rahmenziel 2 & $\begin{array}{l}\text { Bis März 2018 gibt es eine funktionierende Zusteue- } \\
\text { rung zu Integrationskursen innerhalb von 6 Wochen }\end{array}$ \\
\hline Ergebnisziel 2.1 & $\begin{array}{l}\text { Informationswege zwischen verpflichtender Stelle } \\
\text { Jobcenter aktiv bzw. verpflichtender Stelle Asylleis- } \\
\text { tungsgewährung und I-Kursträgern sind formell fest- } \\
\text { gelegt }\end{array}$ \\
\hline Ergebnisziel 2.2 & $\begin{array}{l}\text { Die verpflichtenden Stellen sind über die lau-fenden } \\
\text { und zukünftigen I-Kurse informiert }\end{array}$ \\
\hline Rahmenziel 3 & $\begin{array}{l}\text { Berechtigte Nutzer sind motiviert und in die Lage } \\
\text { versetzt Integrationskurse zu besuchen }\end{array}$ \\
\hline Ergebnisziel 3.1 & $\begin{array}{l}\text { Die Wünsche und Bedarfe der Zuwanderer sind be- } \\
\text { kannt }\end{array}$ \\
\hline Ergebnisziel 3.2 & $\begin{array}{l}\text { Eingliederungsvereinbarung ist verbindlich zwi- } \\
\text { schen Nutzer, SGB II oder Asylleistungsgewährung } \\
\text { geschlossen }\end{array}$ \\
\hline Ergebnisziel 3.4 & $\begin{array}{l}\text { Es besteht eine strukturierte Vernetzung zwischen } \\
\text { den beteiligten Akteuren } \\
\text { können genutzt werden }\end{array}$ \\
\hline
\end{tabular}


Die jeweiligen Ergebnisziele wurden dann in Projektgruppen bearbeitet. Diese Projektgruppen haben u. a. die Schwerpunkte „Informationsaustausch Integrationskursträger“ und „Nutzerverhalten“ und sind entsprechend mit relevanten Akteuren besetzt. ${ }^{39} \mathrm{Zu}$ Beginn werden die drei in den Arbeitsgruppen behandelten und rekonstruierten Fälle kurz ,aufgefrischt ${ }^{\star}$, um anschließend entlang vier durch die Koordinatorinnen und Koordinatoren vorbereitete Fragestellungen bearbeitet zu werden. Die Akteure werden hierfür in zwei Gruppen aufgeteilt, die Ergebnisse an Flipcharts angebracht.

Ein Ausschnitt der Arbeit der Projektgruppe Nutzerverhalten sieht wie folgt aus:

1. Welche Vorteile/Nachteile sieht der Nutzer in der Teilnahme am Integrationskurs?

\begin{tabular}{|l|l|}
\hline $\begin{array}{l}\text { Vorteile/ } \\
\text { motivationsfördernd }\end{array}$ & $\begin{array}{l}\text { Nachteile/ } \\
\text { motivationshemmend }\end{array}$ \\
\hline Spracherwerb/Deutsch lernen & $\begin{array}{l}\text { Neuorganisation der Rolle der Frau- } \\
\text { en; bestehende Rollenverhältnisse } \\
\text { müssen aufgelöst werden im Kurs, } \\
\text { aber auch bereits im Vorfeld in der } \\
\text { Familie; Frau ist nicht nur "für Kin- } \\
\text { der und Herd“ zuständig }\end{array}$ \\
\hline $\begin{array}{l}\text { Gefühlter Fortschritt, "es geht wei- } \\
\text { ter“, Möglichkeiten auf Bleibeper- } \\
\text { spektive, Asylverfahren }\end{array}$ & Zeitverschwendung \\
\hline $\begin{array}{l}\text { Teilhabe an der Gesellschaft } \\
\text { Unterstützung der eigenen Kinder, } \\
\text { bspw. durch Teilnahme am Eltern- } \\
\text { abend }\end{array}$ & $\begin{array}{l}\text { Gesichtsverlust; gemischte Gruppen } \\
\text { (männlich, weiblich), Frau lernt ggf. } \\
\text { schneller; Nationalitätenkonflikt }\end{array}$ \\
\hline $\begin{array}{l}\text { Strukturierter Tagesablauf, Antrieb, } \\
\text { Ablenkung }\end{array}$ & $\begin{array}{l}\text { Neuorganisation von Gewohnheiten/ } \\
\text { alltäglichen Abläufen }\end{array}$ \\
\hline $\begin{array}{l}\text { Kontaktbörse, Kontakte und soziales } \\
\text { Netzwerk entsteht }\end{array}$ & Arbeit statt Sprachkurs \\
\hline
\end{tabular}

39 Das in den Abschnitten 4.4ff. vorgeschlagene Vorgehen sieht vor, vor, die Nutzerperspektive durch vorhandene Fachkräfte darzustellen. Eine Beteiligung von Nutzerinnen und Nutzern ist auch möglich. In Z. wurde das durch die Durchführung eines Workshops „Positive Integrationsgeschichte“ umgesetzt, allerdings nach der Festlegung der Ziele. 


\begin{tabular}{|l|l|}
\hline $\begin{array}{l}\text { Vorteile/ } \\
\text { motivationsfördernd }\end{array}$ & $\begin{array}{l}\text { Nachteile/ } \\
\text { motivationshemmend }\end{array}$ \\
\hline $\begin{array}{l}\text { Rechtsstatus, bspw. nach Abschluss I- } \\
\text { Kurs Inanspruchnahme Niederlas- } \\
\text { sung }\end{array}$ & Finanzielle Nachteile (Selbstzahler) \\
\hline Arbeitsaufnahme & \\
\hline $\begin{array}{l}\text { Verbesserung der Chancen: Arbeit, } \\
\text { Wohnung, Ausbildung, quasi alles }\end{array}$ & Überforderung; Versagensangst \\
\hline
\end{tabular}

2. Welche Voraussetzungen/Bedingungen müssen für den Nutzer erfüllt sein, um den Integrationskurs zu besuchen?

Berechtigung ist Grundvoraussetzung.

Motivation.

Aufklärung, was kann man mit dem Kurs erreichen, Rechte und Pflichten, was bringt mir der Kurs, ...Besonders wichtig bei Personen, die "schulfern", "bildungsfern" sind, ggf. kostenlose Nachhilfe anbieten.

Familiäre Situation muss passen; möglichst wenig Probleme in der Familie, geregelte Kinderbetreuung, Vertrauen in das System, ...

Geeignetes Zeitmanagement: Kindergarten/KiTa-Zeiten, Schulzeiten, eigene Arbeitszeiten; die persönliche Zeitgestaltung, kulturelle und religiöse Anforderungen (Bsp. Mittagsgebet) Hilfestellung bei der Problembewältigung.

Gesundheit.

Mobilität, gute örtliche Erreichbarkeit.

Geeignetes Zeitmanagement: Kindergarten/KiTa-Zeiten, Schulzeiten, eigene Arbeitszeiten; die persönliche Zeitgestaltung, kulturelle und religiöse Anforderungen (Bsp. Mittagsgebet) Hilfestellung bei der Problembewältigung.

3. Was motiviert den Nutzer, einen Integrationskurs zu besuchen? Was hemmt ihn?

\begin{tabular}{|l|l|}
\hline Motivationsfördernd & Demotivierend \\
\hline Teilhabe an der Gesellschaft & Gesundheit \\
\hline & Fehlende Kinderbetreuung \\
\hline & Rollenverständnis; Frau sieht sich als \\
& Hausfrau \\
\hline & Überforderung mit Bildung \\
\hline & Fehlende Mobilität \\
\hline
\end{tabular}




\begin{tabular}{|l|l|}
\hline Motivationsfördernd & Demotivierend \\
\hline & Alter, Ü50 \\
\hline & $\begin{array}{l}\text { Bei langen Wartezeiten ist das Leben } \\
\text { bereits eingerichtet, deutsch gelernt }\end{array}$ \\
\hline
\end{tabular}

4. Welche Handlungsoptionen hat der Nutzer?

\begin{tabular}{|l|l|}
\hline Eigeninitiative & Hemmnisse \\
\hline $\begin{array}{l}\text { Aktive Anmeldung, pünktlich bzw. } \\
\text { im Zeitfenster. }\end{array}$ & $\begin{array}{l}\text { Nichts machen; mangelnde Bereit- } \\
\text { schaft. }\end{array}$ \\
\hline Teilnahme am Sprachkurs. & $\begin{array}{l}\text { Zeitpuffer“ erarbeiten um sich dem I- } \\
\text { Kurs zu entziehen: Krankheitszeiten, } \\
\text { Rausschmiss. }\end{array}$ \\
\hline Freie Trägerwahl, Beschleunigung. & Freie Trägerwahl, Verzögerung. \\
\hline $\begin{array}{l}\text { Aktive Beteiligung an Angeboten, } \\
\text { bspw. Sprachcafé. }\end{array}$ & Schwangerschaft. \\
\hline $\begin{array}{l}\text { Bemühungen und Aktivität für die } \\
\text { bei Kinderbetreuung, Kindergarten, } \\
\text { Haushalt, Tagesmutter, MS-Card. }\end{array}$ & $\begin{array}{l}\text { Behandlung/Arbeitsunfähigkeit ein- } \\
\text { reichen. }\end{array}$ \\
\hline Beratung einholen. & $\begin{array}{l}\text { Boykott des Kurses; mangelnde Be- } \\
\text { reitschaft. }\end{array}$ \\
\hline Kündigung der Arbeit/Schulabbruch. & $\begin{array}{l}\text { Kündigung der Arbeit/Schulabbruch } \\
\text { bspw. bei Schulbesuch, kurz vor Prü- } \\
\text { fung. }\end{array}$ \\
\hline
\end{tabular}




\section{Anregungen zur Ausgestaltung von Case Management ${ }^{40} \mathrm{im}$ Rahmen des kommunalen Integrationsmanagements}

\subsection{Vorbemerkung}

Die in Kapitel 4 ausführlich diskutierten Netzwerkstrukturen sind nicht nur eine Voraussetzung für effektives rechtskreisübergreifendes Case Management, sondern umgekehrt unterstützt Case Management im Einzelfall den Auf- und Ausbau von Netzwerkstrukturen, indem es

- (anonymisierte) Einzelfälle so aufbereitet, dass sie genutzt werden können, um im Netzwerk die Entwicklung gemeinsamer Sinn- und Wertstrukturen empirisch gesichert zu unterstützen;

- über das Monitoring und die Evaluation von Einzelfällen dazu beiträgt, Versorgungslücken und -defizite aufzudecken;

- Daten generiert, die dann - in aggregierter Form - in ein spezifisches Berichtswesen einfließen, das es erlaubt, Zuwanderungsprozesse und ihre Auswirkungen auf die existierende Versorgungsstruktur abzubilden.

Um diese Funktionen zu erfüllen und damit ein Scharnier zwischen Einzelfall und Struktur zu bilden, müssen organisatorisch zwei Voraussetzungen geschaffen werden:

- Ein einheitliches Dokumentationssystem, das es erlaubt, Daten aus unterschiedlichen Case Management-Verläufen zusammenzufassen und organisationsübergreifend zu analysieren sowie

- regelmäßig stattfindende Hilfeplan- bzw. Fallkonferenzen, in denen die Perspektiven mehrerer Akteure aus unterschiedlichen Organisationseinheiten zueinander in Bezug gesetzt werden und ein komplexes Bild von Einzelfällen gewonnen werden kann. Diese bilden dann die Grundlage für Planungsprozesse.

Im Folgenden wird zunächst eine Definition von Case Management als Handlungskonzept gegeben (Abschnitt 5.2), um daran anknüpfend den Kontext des Konzepts und seine spezifische Modifikation als „rechtskreisübergreifendes Case Management" darzustellen (Abschnitt 5.3). Im vierten Abschnitt (5.4) wird der Kreislauf des Case Managements im Detail be-

40 Dieses Kapitel stellt die stark gekürzte und gründlich überarbeitete Fassung der Veröffentlichung von Reis/Hobusch/Kolbe (2011) dar. 
schrieben, wie er auch dem rechtskreisübergreifenden Case Management zu Grunde liegt. Rechtskreisübergreifendes Case Management beinhaltet wie jedes Case Management eine Interaktionsbeziehung zwischen Case Manager und Adressaten. Deren Grundzüge werden in Abschnitt 5.5 skizziert, wobei auch Beispiele für Gesprächsführung diskutiert werden. Nach einem Ausblick in Abschnitt 5.6 folgen in Abschnitt 5.7 Beispiele aus Projektkommunen.

\subsection{Case Management-erste Definitionen}

Bereits seit den 1980er Jahren existiert Case Management in den USA als Handlungskonzept in der Gesundheitsversorgung, auch wenn die Wurzeln noch weiter zurückreichen (vgl. Zander 2000). Seit Ende der 1990er Jahre wurde Case Management in der deutschen Sozialarbeit rezipiert (vgl. Wendt 1997), ohne dass damit zunächst starke Impulse für die Praxis in der Sozial- und Jugendhilfe ausgegangen wären. Insgesamt schien der Eindruck von Ewers und Schaeffer gerechtfertigt, dass die Diskussion des Case Managements in Deutschland von einer deutlichen Kluft zwischen hohen Erwartungen einer- und einer wenig gründlichen Auseinandersetzung mit den Kerngedanken andererseits geprägt war (vgl. Ewers/Schaeffer 2000). Dies änderte sich allerdings rasch: Sowohl im Bereich der Altenhilfe, Pflege und generell des Gesundheitswesens als auch im Feld der Beschäftigungsförderung wurde der Grundgedanke des Case Managements aufgenommen, um zum einen bedarfsgerecht, zum anderen effektiv und effizient Unterstützung bereitzustellen und zu organisieren. Ihren institutionellen Ausdruck fand diese Entwicklung in der 2005 erfolgten Gründung einer „Deutschen Gesellschaft für Care und Case Management“ (DGCC), die die verschiedenen Praxisfelder abdecken soll. Trotz des Booms bleiben aber eine Reihe von Fragen offen. Zwar hat sich die Fachdiskussion über die Grenzen der Praxisfelder auf die Konturen dessen, was als „Case Management" gelten soll, weitgehend geeinigt (vgl. insbesondere Wendt 2005; Monzer 2013; für den Bereich des „Fallmanagements“: Deutscher Verein 2004; 2009; Bundesagentur für Arbeit 2005), doch bestehen in der Praxis selbst nach wie vor viele Unklarheiten.

Deshalb empfiehlt es sich, diesen Leitfaden mit ersten Definitionen zu beginnen. Obwohl auch in den USA nicht von dem Case Management gesprochen werden kann, sondern sich vielmehr unterschiedliche konzeptionelle Strömungen unter diesem Begriff zusammenfassen lassen, ist es möglich, an einer Begriffsdefinition anzusetzen, die ihre Wurzeln im US-ameri- 
kanischen Gesundheitssektor hat, um dann Kernelemente des Case Managements zu bestimmen und notwendige Differenzierungen vorzunehmen. Die „Case Management Society of America“ (CMSA) definiert Case Management „als einen kooperativen Prozess, in dem Versorgungsangelegenheiten und Dienstleistungen erhoben, geplant, implementiert, koordiniert, überwacht und evaluiert werden, um so den individuellen Versorgungsbedarf eines Patienten mittels Kommunikation und verfügbarer Ressourcen abzudecken" (CMSA 1995: S. 69). Aus dieser Definition können die beiden Pole des Case Managements gut erschlossen werden: Die Orientierung am individuellen Bedarf einer- sowie die Planung und Steuerung eines Versorgungsangebots andererseits. Eine weitere auf das Handlungsfeld der Sozialen Arbeit bezogene Definition bringt dies noch deutlicher zum Ausdruck: „Case Management ist ein interpersonaler Prozess, der auf einer Beziehung zwischen einem Case Manager und einem Klienten(-system) beruht. Durch sorgfältig entwickelte Versorgungspläne ist intendiert, die funktionellen Kapazitäten von Individuen und ihrer sozialen Netzwerke, die einen Bedarf an langfristiger Unterstützung haben, zu erhöhen (...) und die effektive Erbringung einer großen Spannbreite an Dienstleistungen über ein Versorgungskontinuum hinweg zu ermöglichen und sicherzustellen (...). Dies (...) bedeutet den Aufbau von Unterstützungsnetzwerken, die Koordination von Dienstleistungen auf der Klientenebene und die Realisierung von Effektivität auf der Systemebene" (Greene 1992: S. 17, zitiert und übersetzt von Ewers 2000b: S. 56f.). Die DGCC, das deutsche Pendant zur CMSA, knüpft an diesen Definitionen an und gibt folgende „offizielle Definition“: „Case Management ist eine Verfahrensweise in Humandiensten und ihrer Organisation zu dem Zweck, bedarfsentsprechend im Einzelfall eine nötige Unterstützung, Behandlung, Begleitung, Förderung und Versorgung von Menschen angemessen zu bewerkstelligen. Das Handlungskonzept ist zugleich ein Programm, nach dem Leistungsprozesse in einem System der Versorgung und in einzelnen Bereichen des Sozialund Gesundheitswesens effektiv und effizient gesteuert werden können“ (DGCC 2012).

Case Management enthält somit die persönliche Interaktion als ein zentrales Element und setzt demzufolge ein funktionierendes „Arbeitsbündnis" zwischen Case Manager und Adressatinnen und Adressaten voraus. Im Unterschied zur klassischen Einzelfallhilfe wird diese Aufgabe ergänzt durch die Koordination von Sach- und Dienstleistungen als ein zweites Element. Hilfe wird nicht (nur) direkt erbracht, sondern vermittelt. Damit diese Vermittlung aber optimal erfolgt, ist ein professionell gestalteter interpersoneller Prozess nötig, der den/die Case Manager zum „Insider-Ex- 
perten“ (Lamb/Stempel 2000: S. 166) macht, d. h. zu einer Person, die sowohl die Binnenperspektive der Adressatin bzw. des Adressaten als auch die Außenperspektive des Versorgungssystems einnehmen und beide vermitteln kann.

Gegenstand des Case Managements ist es auch - dies stellt die zweite wichtige Dimension der zitierten Definition dar - nicht nur professionelle Dienstleistungen zu erschließen, sondern Unterstützungsnetzwerke in der "Lebenswelt" der Adressaten zu identifizieren und zu aktivieren, d. h. „Netzwerke“ auf verschiedenen Ebenen zu knüpfen und für den Einzelfall nutzbar zu machen. Dies beinhaltet eine planerische und sozialräumliche Ausrichtung des Case Managements. Case Management orientiert sich demnach an und arbeitet mit

- den Bedürfnissen der Adressatin bzw. des Adressaten,

- den Strukturen des Versorgungssystems und

- den Potenzialen des Sozialraums.

Idealerweise sollen diese drei unterschiedlichen Orientierungen zu Gunsten der Versorgungsnotwendigkeiten des Einzelfalls integriert werden; in der Realität werden jedoch unterschiedliche konzeptionelle Akzente gesetzt, so dass sich je nach Handlungsfeld diverse Case Management-Varianten unterscheiden lassen (vgl. Löcherbach/Mennemann/Hermsen 2009; Löcherbach et al. 2018).

Deshalb ist es wichtig, das Proprium des Case Managements, den Kern eines arbeitsfeldübergreifenden Handlungskonzepts, herauszustellen. Dieses besteht darin, dass Case Management „in Anlehnung an die Zielvorstellung einer kontinuierlichen und integrierten Versorgung (continuum of care) die zeitlichen und räumlichen Dimensionen des Versorgungsgeschehens überbrückt" (Ewers 2000b: S. 54). Case Management agiert entlang eines zeitlich definierbaren Versorgungsbedarfs kontinuierlich („over time") und koordiniert Hilfsangebote quer zu bestehenden Grenzen von Ämtern, Diensten und Einrichtungen („across services“). Der zentrale Begriff lautet „Versorgungskontinuität“ (ebd.: S. 55). Jenseits des Spannungsfelds zwischen Adressaten („guarding the patients“, vgl. Ewers/Schaeffer 2000: S. 20) und dem Versorgungssystem ("guarding the dollars") muss Case Management die Aufgabe wahrnehmen, die Zersplitterung vorhandener Angebote aufzuheben bzw. zu kompensieren, indem eine Koordinationsleistung vollbracht wird, die mehr ist als der Hinweis auf bestehende Dienste und Einrichtungen: Orientiert an den Gegebenheiten des Einzelfalls werden geeignete Angebote ausgewählt, vermittelt und darüber hinaus verbindlich gesteuert. Im Grad der Verbindlichkeit unterscheiden sich Case Management-Konzepte ebenso wie in der Frage der vorrangigen 
Ausrichtung am Einzelfall oder System - ein Minimum an Sicherung der Versorgungskontinuität, d. h. „Schnittstellenmanagement“, darf jedoch nicht unterschritten werden, wenn noch von "Case Management" die Rede sein soll. ${ }^{41}$ Allerdings bleibt zu beachten, dass die Herstellung stabiler Netzwerkstrukturen, die letztlich Voraussetzung für alle Case Management-Varianten sind, nicht Aufgabe der Case Manager sein kann, sondern auf der Organisationsebene erfolgen muss. ${ }^{42}$ Jedes Case Management muss in der Zeitachse Kontinuität aufweisen (,aus einer Hand“ erfolgen) und unterschiedliche Hilfen und Leistungen koordinieren (und damit zumindest im Auge behalten) - dies ist die Messlatte, an der sich Case Management-Konzepte, die in der professionellen Tradition stehen, und Angebote, die schlicht auf einer Modewelle mitreiten, unterscheiden lassen. Ein weiteres Kennzeichen des Case Managements ist, dass die „Versorgungskontinuität" nicht nur professionelle Angebote („services“) umfasst, sondern sich auch auf Ressourcen im sozialen Umfeld der Adressatin bzw. des Adressaten erstrecken soll. Der „Fall“, der Gegenstand des Case Managements ist, wird als „sozialer Fall“ betrachtet. Das bedeutet, dass das Netzwerk, in dem die Adressatin bzw. der Adressat lebt, nicht nur in der Problemanalyse rekonstruiert, sondern auch in die Lösungsstrategie einbezogen wird. ${ }^{43}$ Die Definition der CMSA stellt die „Vermittlungsleistung“ des Case Managements in den Vordergrund, über die Versorgungskontinuität gesichert wird. Sie hebt darauf ab, dass es sich bei Case Management um ein Handlungskonzept handelt, das in unterschiedlichen Arbeitsfeldern des Sozial- und Gesundheitswesens genutzt werden kann, und ist dadurch notwendigerweise allgemein. Im Weiteren wird es darum gehen, den Kontext des Einsatzes zu diskutieren und dabei die Eckpunkte zu benennen, die geklärt sein müssen, wenn Case Management tatsächlich als Konzept

41 Aus diesem Grund umfasst Case Management immer auch Hilfeplanung, aber nicht bei jeder Hilfeplanung handelt es sich um Case Management. Denn bei Hilfeplanung ist durchaus offen, ob die „geplanten“ Hilfen auch von der Hilfeplanerin bzw. dem Hilfeplaner koordiniert, überwacht oder gar gesteuert werden oder aber die entscheidende Vermittlungsleistung von der Adressatin bzw. vom Adressaten wahrgenommen werden muss, die bzw. der von Dienst zu Dienst „weitergereicht" wird.

42 Vgl. hierzu Kapitel 4 dieses Leitfadens.

43 Natürlich gibt es deutliche Unterschiede zwischen den einzelnen Konzepten des Case Managements in der Frage, in welchem Ausmaß das persönliche „Netzwerk" nicht nur rhetorisch beschworen, sondern tatsächlich zum Anlass für „Netzwerkarbeit" mit dem Umfeld der Adressatinnen und Adressaten wird. Die sozialräumlichen Aspekte des Case Managements werden insbesondere im „Nijmeger Modell“ hervorgehoben (vgl. Oostrik/Steenbergen 2000). 
eingesetzt werden soll. Es geht somit um die Grundstrukturen einer Kontext- und Konzeptanalyse, die eine Konkretion des abstrakten Konzepts erlaubt und dieses für einzelne Arbeitsfelder spezifiziert.

\subsection{Kontext und Konzepte von Case Management}

\subsubsection{Case Management - ein Konzept und seine Varianten}

Die deutsche Rezeption des Case Managements vermittelt teilweise das Bild eines zumindest in den Grundzügen widerspruchsfreien Konzepts. ${ }^{44}$ Demgegenüber versucht insbesondere David Moxley (1997) zu zeigen, dass sich im Case Management die zentralen Widersprüche eines einzelfallbezogenen öffentlich finanzierten Versorgungssystems offenbaren: Bedarfsgerechtigkeit ist nicht ohne weiteres mit der Knappheit verfügbarer Ressourcen vereinbar. Diese Ambivalenz mündet in eine Vielzahl von Konzeptvarianten, die mal stärker auf die Bedürfnisse der adressierten Personen mal mehr auf die Effektivierung des Versorgungssystems gerichtet sind. All diese Varianten können als Versuche angesehen werden, das „case manager's dilemma“ zu lösen (vgl. Ewers 2000b: S. 71): Case Manager sollen individuelle Bedürfnisse einer- und Versorgungsstrukturen andererseits so miteinander vermitteln, dass eine dem Einzelfall „angemessene“ Versorgung gewährleistet ist. Da es kein klares Kriterium für diese Angemessenheit gibt, stehen Case Manager immer vor der Aufgabe des „Aushandelns“, und zwar in dreifacher Form: Es müssen Ziele, einzelne Maßnahmen und Fristen der Zielerreichung ausgehandelt werden und zwar so, dass die Adressatinnen und Adressaten motiviert bleiben, den Prozess des Case Managements als einen ihren Interessen dienlichen anzusehen, und gleichzeitig die Systeminteressen gewahrt bleiben. Im Case Management reproduziert sich somit der Widerspruch, der der Sozialen Arbeit immer schon anhaftet, und der bereits vor über dreißig Jahren als „doppeltes Mandat" ausbuchstabiert wurde (vgl. Böhnisch/Lösch 1973: S. 28). Allerdings betrifft er nicht nur die Soziale Arbeit, sondern stellt sich als spezifisches, praktisch zu lösendes Handlungsproblem auch in der Sachbearbeitung von Sozialverwaltungen: „Jeweils konkrete und widersprüchliche, komplexe Handlungs- und Entscheidungssituationen im Einzelfall sachangemessen, das heißt unter Würdigung der Besonderheit des jeweiligen Falles, mit den bürokratischen Verfahrensweisen in Einklang zu bringen, ohne dass hierbei

44 Vgl. Wendt 1991 und 1997, differenzierter dagegen Ewers (2000a). 
nur ein voreingerichteter standardisierter Ablauf abgearbeitet werden könnte“ (von Harrach/Loer/Schmidtke 2000: S. 90). Dieser Widerspruch, der öffentlich organisierten personenbezogenen Dienstleistungen innezuwohnen scheint, nimmt für einzelne Handlungsformen jeweils eine spezifische Form an. So kann im Case Management das genannte Handlungsproblem analytisch in drei Dilemmata ausdifferenziert werden (vgl. Oostrik/Steenbergen 2000: S. 257f.):

- Das „strategische Dilemma“, das darin besteht, dass die Case Manager mit den Adressatinnen und Adressaten Ziele vereinbaren wollen, die Ziele der adressierten Personen aber nicht unbedingt mit den Zielen des Anstellungsträgers oder auch der Case Manager übereinstimmen.

- Das „instrumentelle Dilemma“ zwischen der Tatsache, dass das Case Management aus Regeln und Vereinbarungen besteht (s. u.), ein starres Festhalten an diesen Regeln unter Umstanden aber zu unflexibel für den Einzelfall ist, und von daher eine Revision der Regeln die „angemessenere" Variante darstellen kann.

- Das „narrative Dilemma“:45 Unter den Teilnehmenden des Case Managements kann es zu unterschiedlichen Interpretationen von Ereignissen mit entsprechend differierenden Konsequenzen kommen. Es ist die herausfordernde Aufgabe der Case Manager, sich in die Interpretation der Adressatinnen und Adressaten einzufühlen, gleichzeitig aber einen - von dieser unter Umständen abweichenden - gemeinsamen Interpretationsrahmen anzustreben.

Diese Dilemmata erhalten im Rahmen des Case Managements deshalb ihre besondere Brisanz, weil dessen Ziel ausdrücklich die Vermittlung der beiden Pole ist und Case Management nur dann seine Ziele erreichen kann, wenn das Handlungsproblem bewältigt wird. Um das „case manager's dilemma" präziser beschreiben zu können, unterscheidet Moxley zwei grundlegende Typen des Case Managements, über die sich das Dilemma weiter bearbeiten lässt (er nennt sie „Archetypen“). Diese markieren Pole einer denkbaren Konzeption von Case Management, in denen sich die beiden grundlegenden Orientierungen („System“ vs. Adressaten) in Reinform auskristallisieren:

45 Oostrik/Steenbergen (2000) gehen davon aus, dass Case Manager sich immer mit den Deutungen und Interpretationen (also „Erzählungen“, was nicht abwertend gemeint ist) auseinandersetzen müssen: „Der Case Manager muss sich die Frage stellen, wie die Deutung eines Lebenslaufes (...) mit diesem ,Erzähler' ausgehandelt werden kann" (Oostrik/Steenbergen 2000: S. 256). Deshalb bezeichnen sie dieses Dilemma als „narrativ“. 


\subsubsection{1 „Consumer-driven“ Case Management}

Diese Form des Case Managements zielt darauf, Adressatinnen und Adressaten dabei zu unterstützen, ihre Bedürfnisse zu artikulieren und ihnen den Zugang zum Angebot sozialer Dienstleistungen zu eröffnen: „Case Management, das auf diesem Archetyp basiert, passt sich an die Konsumenten an und hilft den Menschen, jene Unterstützungen und Angebote zu organisieren, die am meisten den Bedürfnissen entsprechen, wie sie von ihnen selbst definiert werden“ (Moxley 1997: S. 20). Dieser Typus achtet zwar auch auf Kosten, betrachtet aber Menschen nicht als Kostenquelle, weder in Bezug auf ihr Verhalten noch auf unmittelbare Ausgaben (vgl. ebd.: S. 21). Das zentrale Ziel des „consumer-driven“ Case Managements ist die „Personalisierung“. Dies bedeutet, sowohl persönliche Stärken der Adressatin oder des Adressaten zu wecken und zu stützen als auch formelle wie informelle Ressourcen daran auszurichten: „Personalisierung meint in diesem Zusammenhang die Förderung der persönlichen Ressourcen der Kundschaft. Sie beruht auf einem Verständnis der Person als einem kulturellen und sozialen Wesen, das versucht, Kontrolle über umgebungsbedingte Situationen zu erlangen, die sehr anspruchsvoll und vielleicht sogar feindlich sein können. Das Ziel der Personalisierung verlangt vom Case Management, eine Person als selbstbestimmten Akteur zu verstehen“ (ebd.).

Dem entspricht ein an der Person der Adressatin oder des Adressaten ausgerichtetes Zielsystem:

- Verantwortlichkeit gegenüber den artikulierten Bedürfnissen, also „den Kunden dabei helfen, ihre eigenen Bedürfnisse zu erkennen und ihre Wünsche auszudrücken“ (ebd.: S. 24).

- Herstellung einer unterstützenden Beziehung („Supportive Alliance“).

- Zuschneiden der Hilfsangebote angemessen an die spezifische Situation der Adressatin oder des Adressaten: „Dieser Archetyp (...) verknüpft die Personalisierung mit dem Unterstützungsangebot und fordert die Anpassung der Unterstützung an die spezifische Situation der Leistungsadressaten“ (ebd.).

\subsubsection{2 „System-driven“ Case Management}

Diese Form des Case Managements „verkörpert jene Werthaltung, die den Ansatz von Effizienz und Effektivität in den Vordergrund des Umgangs 
mit personenbezogenen Dienstleistungen stellt" (ebd.: S. 15$)^{46}$ und baut auf der Grundidee der „Kontrolle” auf. Kontrolle wird dabei doppelt gefasst:

- Als Kontrolle des „Systems“, z. B. über den sparsamen Umgang mit knappen Ressourcen.

- Als Kontrolle von Personen, um zu verhindern, dass sie durch (potenziell) abweichendes Verhalten aus dem Versorgungssystem fallen und sich unkontrolliert gegen die Gesellschaft wenden.

„Die Integrität des sozialen Systems ist das primäre Ziel dieses Archetyps (...). Seine zentrale Absicht (...) liegt in der sozialen Kontrolle: In diesem Fall ist Case Management bestrebt, die Kontrolle von Menschen durch ein System zu verstärken, so dass die Kosten, die durch Ineffektivität und abweichendes oder unerwünschtes Verhalten entstehen, reduziert oder zumindest besser gemanagt werden“ (ebd.: S. 16). Die Case Manager übernehmen die Rolle des „Treuhänders“ (trustee) für die Gesellschaft gegenüber der Adressatin bzw. des Adressaten. Die Grundidee der Kontrolle prägt das Zielsystem dieser Form des Case Managements:

- Koordination zersplitterter Angebote und Effizienzsteigerungen auf Systemebene.

- Rationierung von Leistungen: „Rationierung kann Kosten auf den Konsumenten verlagern und die Fürsorgelast an informelle Träger und an Dienste außerhalb eines professionellen Netzwerks abgeben" (ebd.: S. 17).

- Auf Adressatenverhalten bezogene Kostenkontrolle: Ihr Ziel ist, „zu versuchen, die Kosten herunterzufahren oder zu kontrollieren, die durch eine intensive Nutzung professioneller Angebote hervorgerufen wurden, indem sie den Bedürfnissen der Individuen mit innovativen Arrangements entgegnen, die auf das Angebot des Gemeinwesens und auf integrierte Haushaltsunterstützung fokussiert sind (...) (ebd.: S. 19).

- Ersatz teurer durch billigere Leistungen (auch durch Anbahnung informeller Unterstützung).

Die beiden Archetypen basieren auf unterschiedlichen konkurrierenden Werten und Menschenbildern. Obwohl dies in der Praxis eine Quelle von Rollenkonflikten darstellt, werden beide Archetypen in der Praxis nicht selten miteinander vermischt, was u. a. aus widersprüchlichen politischen Aufträgen resultiert - bspw. darin, dass die Organisation dazu auffordert,

46 Die Zitate anglo-amerikanischer Literatur in diesem Kapitel wurden von Laura Reis übersetzt. Ihr sei an dieser Stelle gedankt. 
die adressierten Personen als Konsumierende zu betrachten, ihnen zugleich jedoch lediglich ein eingeschränktes Leistungsangebot zu offerieren ist. Eine vergleichbare Situation stellt sich im Kontext des Postulats „passgenauer Hilfe“. Dieses wäre als Ausformung eines „consumer-driven“ Case Managements zu verstehen, ginge damit nicht gleichzeitig die explizite Erwartung einher, Ausgaben zu reduzieren. Umgekehrt ist nur schwer vorstellbar, wie das Ziel des „system-driven“ Case Managements, abweichendes Verhalten zu „kontrollieren“, erreicht werden kann, ohne nicht zumindest ein Minimum an personenbezogener Hilfeleistung zu erbringen. Erst eine genauere Analyse der Praxis des Case Managements kann Aufschluss darüber geben, wie stark die skizzierte Zwiespältigkeit des Case Managements das Handeln der Case Manager beeinflusst, und in welche Richtung sie aufgelöst wird. Eine solche Analyse sollte sich zunächst dem Kontext widmen, in dem das Case Management eingesetzt wird, um dann vor dem Hintergrund der Klärung des Auftrags konzeptionelle Fragen zu diskutieren, die das Leistungsangebot bestimmen.

\subsubsection{Der Kontext des Case Managements und seine Analyse}

Die beiden elementaren Merkmale des Case Managements, sowohl „across services“ zu wirken, als auch „over time“ anzusetzen, werden unterschiedlich ausgeformt, je nachdem, wie der Kontext gestaltet ist, in dem Case Management ansetzt (vgl. zum Folgenden Moxley 1997). Deshalb besteht der erste Schritt der Modellierung eines einzelfallbezogenen Case Managements in der einzelfallübergreifenden und -unabhängigen Analyse dieses „Kontexts“, nämlich der Zielgruppen, der politischen Einbettung und der Angebotsstrukturen sowie der vorhandenen Netzwerke auf der Strukturebene.

\subsubsection{Zielgruppen}

Voraussetzung zur Bestimmung des Kontexts ist eine Analyse der Zielgruppe und der sozialen Probleme, die im Zentrum des Case Managements stehen sollen, ebenso wie die vorhandenen Bewältigungsstrategien und -ressourcen sowie formelle und informelle Unterstützungsnetzwerke. Die Kontextanalyse dient dazu, „die Zielgruppenbevölkerung zu untersuchen und die sozialen Probleme und gesundheitlichen Beschwerden von Menschen zu identifizieren, die das Case Management nutzen werden“" 
(Moxley 1997: S. 44). Dies schließt auch die eingehende Betrachtung der lokalen Lebensbedingungen der Zielgruppe ein, um hieraus einerseits Strategien zur Veränderung dieser Bedingungen abzuleiten und außerdem Erkenntnisse darüber zu gewinnen, wie einzelfallbezogene und -übergreifende Unterstützungspotenziale erschlossen werden könnten. Diese sind ein kritischer Erfolgsfaktor, denn „Case Management-Systeme werden höchstwahrscheinlich so effektiv sein wie ihr Ressourcenkontext" (ebd.: S. 81). Dies wird häufig vernachlässigt: „Es ist sehr ungewohnt für Case Manager, umfangreiche Informationen über die lokalen Verhältnisse und vorhandenen Ressourcenprobleme mit der Absicht zu sammeln, langfristige Strategien zu artikulieren, um Ressourcen zu entwickeln und so das System zu verbessern“ (ebd.: S. 82).

Die genannte Anforderung zu erfüllen, ist alles andere als einfach. Dies liegt nicht nur an „Sprachbarrieren“, sondern an der Komplexität des Phänomens „Migration“. Hierzu muss etwas weiter ausgeholt werden. Die Anwendung des Konzepts Case Management als „rechtskreisübergreifendes Case Management" begründet sich nicht zuletzt dadurch, dass es - wie bereits in der Einleitung dieses Leitfadens festgehalten - den Flüchtling nicht gibt, „dafür sind die (früheren und aktuellen) Lebenslagen der Menschen zu verschieden. Entsprechend kommt es bei der Aufnahme und Versorgung sowie bei Maßnahmen zur Förderung der gesellschaftlichen Teilhabe darauf an, wie gut sie zu den individuellen Lebenslagen passen" (Forschungsbereich beim Sachverständigenrat deutscher Stiftungen für Integration und Migration 2017: S. 6). Gleichzeitig lassen sich aber habituelle Gemeinsamkeiten von Personengruppen identifizieren - das gilt nicht nur für Flüchtlinge, sondern generell. Die Herausforderungen, vor denen viele Zugewanderte stehen, müssen deshalb im Spannungsfeld von „Kollektivschicksal" und individueller Biografie verortet werden. Hierzu ist es sinnvoll, auf das Konzept des „Habitus“ von Pierre Bourdieu zurückzugreifen.

"Sozialisation“ vollzieht sich aus der theoretischen Perspektive Bourdieus dadurch, dass sich die sozialen Akteure auf die Regeln der gesellschaftlichen Felder, in denen sie sich bewegen, einstellen und einen entsprechenden Habitus entwickeln, der es ihnen erlaubt, sich ohne beständige Reflexion im jeweiligen Feld kompetent zu behaupten. Der „Habitus“ „ist ein praktischer Operator, ein Mechanismus, der die Praxis der Struktur anpasst und damit die praktische Reproduktion der Struktur gewährleistet" (Müller 2014: S. 39). Der den Regeln des Feldes entsprechende Habitus ermöglicht es personalen Akteuren, sich in den jeweiligen Feldern erfolgreich zu bewegen, gleichzeitig werden dadurch die Regeln fortgeschrieben. Der Habitus entsteht durch die zum Teil langjährige Inkorpora- 
tion von Wahrnehmungsmustern und Dispositionen, allerdings nicht einfach durch Adaption. Vielmehr „konzeptualisiert er (Bourdieu, Anm. d. Verf.) Inkorporierung statt als soziale Prägung als eine aktive Aneignung der sozialen Welt, und zwar nicht durch ein Nachahmen von ,Vorbildern', sondern durch ein mimetisches Nachmachen der ,Handlungen anderer (Bourdieu 1987: S. 136, Hervorhebung im Original), ihrer Bewegungen, Haltungen und Gesten. Ein solches Nachmachen schließt stets Momente des Andersmachens, des Ausprobierens und regulierten Improvisierens ein (vgl. ebd.: S. 107). Da Bedeutungen, Überzeugungen und Wertvorstellungen nach Bourdieu nur in den öffentlich zugänglichen Vollzügen sozialer Praktiken existieren, kann diese Aneignung nichts anderes sein als die Erfahrungen, die in der sozialen Mimesis der Handlungen anderer gemacht werden (vgl. Krais/Gebauer 2002: S. 61). Aus der Gesamtheit dieser Erfahrungen geht der Habitus hervor" (Alkemeyer/Buschmann 2017: S. 284).

Wie Bourdieu an einigen empirischen Beispielen zeigt (z. B. in seiner Studie über den Heiratsmarkt im Béarn), produziert die „Trägheit“ (hysterisis) des Habitus jedoch immer wieder Ungleichzeitigkeiten zwischen $\mathrm{Ha}-$ bitus und Feldstruktur, die Leid, aber auch Widerstand hervorrufen können. Eine weitere Quelle für Konflikte stellt eine Konstellation dar, die Alkemeyer und Buschmann (2017) analysieren. Zunächst gilt: „Indem sich Menschen in ihrem Lebenslauf fortlaufend zwischen verschiedenen Feldern und Lebensformen bewegen, lernen sie immer auch, fortlaufend wiederkehrende Bewegungen, Körpertechniken und Tätigkeiten reflexiv den Regeln, Anforderungen und Erwartungen des jeweiligen Kontextes anzupassen" (Alkemeyer/Buschmann 2017: S. 287). Hieraus bildet sich der je individuelle, biografisch konstituierte Habitus. Gleichzeitig bildet die Existenz unterschiedlicher Felder mit teilweise verschiedenen habituellen Anforderungen die Grundlage für die Ausbildung von Individualität, auch gegen diese Anforderungen: „In jeder Praktik sind die Teilnehmer verschiedenartig engagiert und positioniert: Sie werden unterschiedlich berührt, bringen verschiedene Erfahrungen und Erwartungen ein, entwickeln aufgrund ihrer jeweiligen körperlichen, mental-sprachlichen und personalen Situiertheit disparate Sichtweisen, Interessen und Wünsche, lassen sich verschieden adressieren und rufen auf disparate Kontexte verweisende ,kognitive Artefakte' (Norman 1993) wie Regeln, Bewertungsmaßstäbe, Wissens- und Rechtfertigungsordnungen als Partizipanden auf, um ihren Sichtweisen Nachdruck, Plausibilität und Legitimität zu verleihen" (Alkemeyer/Buschmann/Michaeler 2015: S. 41). Wenn sich auf diese Weise eine Kluft zwischen der „objektiven“ Feldposition und der subjektiven habituellen Ausrichtung ergibt, öffnet sich der Raum für eine kritische 
Überprüfung - von beidem: „Aus dieser Kluft erwächst die Möglichkeit, alles das, was im Horizont einer Welt als selbstverständlich und unverrückbar erscheint, vor dem Erfahrungshintergrund einer anderen Welt zu prüfen, zu beurteilen und praktisch zu kritisieren, indem materielle Objekte und kognitive Artefakte (Orientierungsrahmen, Beurteilungsmaßstäbe, Rechtfertigungsordnungen etc.) dieser anderen Welt als Partizipanden in eine gegenwärtige Praxis eingebracht werden (vgl. Boltanski/Thévenot 2007): Auf der Grenze zwischen verschiedenen Welten mit ihren je eigenen Verweisungszusammenhängen von Dingen, Artefakten und Handlungen ergeben sich Möglichkeiten zur kritischen Distanznahme, Beurteilung, Bewertung und Grenzbestimmung des je Gegebenen, als deren Träger seit der Neuzeit das Subjekt gilt" (Alke-meyer/Buschmann/Michaeler 2015: S. 45).

Zusammengefasst ergeben sich im Zusammenspiel von Feld und Habitus zwei Krisenpotenziale:

- Der „Hysterisis-Effekt“, hervorgerufen durch die „Trägheit“ habitueller Strukturen gegenüber dem Wandel von Feldstrukturen (oder ganzen sozialen Räumen). Bourdieu gibt in seinen Studien über die Situation der kabylischen Bauern ein eindrucksvolles Beispiel: „Der authentische Bauer muss die bäuerlichen Werte aufrechterhalten, selbst wenn er dem städtischen Leben ausgesetzt ist: Die bäuerliche Gesellschaft, die mit Anerkennung sonst eher sparsam umgeht, ist voll des Lobes für den, der ihre Muster und Normen noch respektiert, weiterhin als Bauer lebt, fühlt, denkt und ,dem Weg seines Vaters und seines Großvaters folgt. ' Man sagt dann: ,Er lebt da wie in der Heimat ““" (Bourdieu 2003: S. 141f.).

- Differenzierte bzw. widersprüchliche habituelle Anforderungen in den verschiedenen Feldern, die von einer Person in Einklang gebracht werden müssen: „Die Selbst-Bildung erfolgt mithin trans-situativ in der Bewältigung situativer, oft widersprüchlicher und damit prinzipiell kritisierbarer Anforderungen. Dies zwingt dazu, fortlaufend Spielräume auszuloten und Spielzüge zu finden, die man sich vorher nicht hätte ausdenken können: Jede ,Lösung' ist immer nur vorläufig, die SelbstBildung kommt zu keinem Ende" (Alkemeyer/Buschmann/Michaeler 2015: S. 41). 
Wie Bourdieu am Beispiel der kabylischen Bauern zeigt, können diese Brüche fatale Folgen für die Betroffenen nach sich ziehen: „Die Umsiedlung ${ }^{47}$, eine der ganzen Gruppe (...) aufgezwungene Emigration geringer Reichweite, berührt das gesamte soziale Leben, weil mit der Organisation des Siedlungsraums auch das darauf beruhende Schema sozialer Strukturen verändert und die Vertrautheit der Individuen mit ihrer Umgebung zerstört wird. Der entwurzelte Bauer, dem die Umgebung, in die er geboren wurde, als einzig vertraute Umgebung gilt und dessen ganzer körperlicher Habitus dem Raum ,angepasst ${ }^{`}$ ist, in dem er sich gewöhnlich bewegt, ist tief in seinem Wesen getroffen, so tief, dass er seiner Verwirrung keine Worte verleihen und noch weniger die Gründe dafür bestimmen kann“ (Bourdieu 2003: S. 180).

Diese Analysen können systematisch genutzt werden, um die Situation von Geflüchteten zu verstehen. Auch hier geht es um habituelle Prägungen und darauf aufbauende Aneignungsstrategien für verschiedene gesellschaftliche Felder, die durch die Flucht (analog zur Zwangsumsiedlung) „entwertet“ werden. Es geht aber nicht allein um die Entwertung institutionalisierten Bildungskapitals oder die Nicht-Akzeptanz des „AndersSeins" - gravierender ist der Verlust habitueller Orientierungen und der Zwang, in kürzester Zeit neue Orientierungen zu finden, eine Aufgabe, auf deren Tragweite auch Oskar Negt in seiner Autobiografie als Flüchtling hingewiesen hat (vgl. Negt 2016). Und der Aufbau neuer Orientierungen geschieht unter der oben skizzierten Voraussetzung, dass die verschiedenen gesellschaftlichen Felder unterschiedliche, ja unter Umständen divergierende Anforderungen stellen. Mit Letzterem sind natürlich alle Gesellschaftsmitglieder konfrontiert und die Konstitution des individuellen Habitus vollzieht sich über das „Navigieren“ zwischen den jeweiligen Feldern. Sie sind „befähigt“, sich kompetent in diesen Feldern zu bewegen. In diesem Kontext bedeutet befähigt, „in die Lage versetzt zu sein, auf im Verlaufe einer Praktik sich etablierende situative Kontexte, Anforderungen und Erwartungen so zu reagieren, dass die Reaktionen als sinnvolle, angemessene und somit kompetente Antworten Anerkennung finden. Umgekehrt lassen sich Individuen allein dann als Teilnehmer einer Praktik engagieren, wenn sie einen ,sozialen Sinn` (Bourdieu 1987) für die jeweilige

471960 waren im Zuge des Algerienkrieges 2.157.000 Algerierinnen und Algerier, ein Viertel der damaligen Gesamtbevölkerung, direkt oder indirekt von „Umsiedlungen“, d. h. Enteignung und Konzen-tration in Massensiedlungen betroffen (vgl. Bourdieu 2003: S. 94). Bourdieu begann seine Karriere als Ethnologe und Soziologe, indem er diesen Prozess und seine Folgen sensibel verfolgte. 
Praktik (erworben) haben und einen ihnen verständlichen Deutungsrahmen erkennen können. Lernen ist vor diesem Hintergrund ein Prozess, der aktive wie passive Momente umfasst und sowohl Möglichkeitsräume eröffnet als auch Widerstände bedingt, die es notwendig machen, sich in der Praxis fortlaufend neu bzw. anders zu organisieren - also stets weiter zu lernen“ (Alkemeyer/Buschmann 2017: S. 277). Diese „Fähigkeit“ korrespondiert aber mit den „objektiven“ Voraussetzungen des Feldes: Die fähigen Spielerinnen und Spieler müssen auch zu dem Spiel zugelassen werden: „Wissensordnungen, Diskurse, Kodes, materielle Strukturen und (Fremd- wie Selbst-)Techniken der Subjektivierung (...) können allgemeine Richtungen für die jeweils historischen Möglichkeiten der Subjektwerdung angeben (...). Das kritische Potenzial dieses praxeologisch informierten Konzepts der Subjektivierung und Selbst-Bildung liegt dabei darin, die Entstehung des Subjekts als in von unterschiedlichsten Machtrelationen und Normierungen geprägten fortlaufenden und konflikthaften Prozessen in den Blick zu nehmen, in denen auch praktische Kritik, Widerständigkeit und Eigensinn ihren Platz haben" (Alke-meyer/Buschmann 2016: S. 133). Zugewanderte und insbesondere Geflüchtete müssen sich diese Fähigkeiten innerhalb kurzer Zeit aneignen und ihnen stehen - wie oben gezeigt - häufig kleinere Möglichkeitsräume zur Verfügung.

Damit ist nicht ausgesagt, dass diese Prozesse einer habituellen Neuorientierung immer extern und intensiv unterstützt werden müssen. Aber es müssen die Möglichkeiten zur Unterstützung vorhanden sein und sie müssen so ausgestaltet sein, dass sie ggf. „prozessbegleitend“ wirken können, auf die Lebenssituation bezogen sind und nicht durch „Zuständigkeitsgrenzen“ (wie Rechtskreiswechsel) behindert werden.

\subsubsection{Politische Einbettung}

Die Kontextanalyse erstreckt sich auch auf den politischen Raum des Case Managements, um hieraus die expliziten und impliziten Aufträge aufzudecken und auf ihre potenzielle Realisierbarkeit hin zu überprüfen. Das Case Management trägt als Instrument potenzielle Widersprüche weiter, die den institutionellen Kontext prägen, in dem es eingesetzt wird: „Case Management spiegelt die wachsenden Kontroversen in der Gesundheitsfürsorge wider, wobei menschliche Würde, Bedeutung und Sensibilität von Dienstleistungen, Kostenkontrolle und Qualitätssicherung thematisiert 
werden“ (Moxley 1997: S. 46). Dies gilt insbesondere für den Einsatz des Case Managements mit dem Ziel der "Integration“. ${ }^{48}$

\subsubsection{Innerorganisatorische Voraussetzungen für Case Management}

Die politische Einbettung verweist direkt auf die Ziele und Struktur der Organisation, in deren Rahmen Case Management angeboten wird.

- Grundsätzlich muss das Case Management im Kontext der Dienste verortet werden, in die es eingebettet ist, um seine Aufgaben bestimmen zu können: Geht es mehr um ein „internes“ Case Management („system-driven“ Case Management, s. o.), das darauf abzielt, vorhandene Dienstleistungen zu vernetzen und zu optimieren: „In diesem Sinne managt der Case Manager die möglichen Angebote, den Weg des Adressaten zu diesen Angeboten und die Behebung auftretender Probleme“ (Moxley 1997: S. 51)? Oder steht die „externe Orientierung“ („consumer-driven Case Management“, s. o.) im Vordergrund: Die Einnahme der Perspektive der Adressatinnen und Adressaten auch dort, wo formelle Angebote fehlen? Die Case Manager treten dann weniger als „Koordinatorinnen und Koordinatoren“, sondern vielmehr als „Ressourcenbeschaffende“ oder „Projektentwickelnde“ auf, die neue Angebote kreieren.

- Kritisch ist das Verhältnis der Case Manager zu anderen Diensten und Einrichtungen. Dabei geht es zunächst um die elementarsten Voraussetzungen wie Schnittstellen innerhalb und zwischen Organisationen und deren Optimierung, weiterhin aber auch um das jeweilige professionelle Selbstverständnis sowie um Menschenbilder und Arbeitsansätze.

- Wenn sich Case Management als Arbeitsansatz ausbreitet, wird die Arbeitsteilung/Verknüpfung zwischen einzelnen, gegeneinander spezialisierten Ansätzen des Case Managements zu einer Aufgabe der Zukunft. Das bedeutet, dass bereits bei der Konzipierung von Case Management zu berücksichtigen ist, in welcher Konstellation allgemeine und spezialisierte Dienste zueinanderstehen.

An allen genannten drei Punkten lässt sich zeigen, dass die Schlüsselfrage für das Case Management lautet, über welche Steuerungskompetenzen es innerhalb eines gegebenen organisationalen Kontexts verfügt und auf welche Weise Steuerungsfunktionen konkret wahrgenommen werden. Damit

Vgl. hierzu ausführlich Kapitel 3. 
Case Management aber überhaupt Steuerungsfunktionen wahrnehmen kann, müssen entsprechende organisatorische Bedingungen geschaffen werden:

- Mit der Einführung des Case Managements muss der Entscheidungsund Gestaltungsspielraum der Mitarbeitenden deutlich ausgedehnt werden. Die „Fallverantwortung“ der Case Manager muss sich organisationsintern in entsprechenden Entscheidungskompetenzen niederschlagen.

- Der Autonomiegewinn muss flankiert und stabilisiert werden durch die Vermittlung einer den Aufgaben entsprechenden Qualifikation.

- Den Mitarbeitenden müssen Partizipationsmöglichkeiten bei der konkreten Ausgestaltung von Planungsprozessen eingeräumt werden - von der Bildung von Qualitätszirkeln bis hin zur Einbeziehung der Mitarbeitenden in die oberste Steuerungsebene der einzelnen Projekte.

- Die hohe Handlungskompetenz der Case Manager muss abgestützt werden durch professionelle Formen fachlicher Unterstützung, z. B. Coaching, Supervision und kollegiale Fallberatung.

- Die Organisationsstrukturen der Träger müssen an die neuen Aufgaben angepasst werden. So ist es notwendig, die Aufnahmekriterien in das Case Management klar zu bestimmen und die Schnittstelle "Zugang“ eindeutig zu gestalten. Auch müssen die Erfolgskriterien für alle Akteure transparent sein. Die Vorgesetzten müssen in der Lage sein, den Prozess des Case Managements zu verstehen und ihre Bewertungskriterien für die Einschätzung der Mitarbeitenden an den Zielen des Case Managements ausrichten.

- Je interessierter die Leitungsebene an der Etablierung der neuen Strukturen ist, ohne unnötigen Druck zu erzeugen, umso ertragreicher ist die Handlungsautonomie für die Gesamtorganisation.

- Die Leitungsebene muss die Implementation kompetent begleiten und insbesondere für die Verknüpfung von Case Management und Netzwerkarbeit sowie Sozialplanung verantwortlich zeichnen.

- Effektives Case Management benötigt funktionierende Angebotsnetze. Das bedeutet, dass qualitativ und quantitativ ausreichende Hilfsangebote existieren müssen oder - wenn sie fehlen - der rasche Aufbau institutionell gesichert ist. Hierzu bedarf es eines Überblicks über die Angebote (z. B. Angebotsdatenbank), gleichzeitig aber auch geeigneter Steuerungsformen (Kontraktmanagement).

- Um den Gesamtprozess des Case Managements zu steuern, ist ein funktionierendes Controlling auf EDV-Basis unerlässlich. 


\subsubsection{Außerorganisatorische Voraussetzungen für Case Management in} der Variante des rechtskreisübergreifenden Case Managements

Case Management setzt die Zusammenarbeit mehrerer Organisationen bzw. Organisationseinheiten voraus. Deshalb muss die organisationsinterne Perspektive durch eine organisationsübergreifende Betrachtung ergänzt werden. Dadurch wird für die Konzipierung von Case Management eine intensive Beschäftigung mit Netzwerken unabdingbar. ${ }^{49}$ Hinsichtlich der Ausgestaltung des rechtskreisübergreifenden Case Managements als spezifischer Form des Case Managements stellen sich einige grundsätzliche Probleme:

\subsection{Rechtskreisübergreifendes Case Management bedarf einer besonderen Indikation}

Da rechtskreisübergreifendes Case Management eine Variante des Handlungskonzepts Case Management darstellt, wird es - analog zu anderen Varianten des Case Managements - nur unter bestimmten Umständen zum Einsatz kommen. Hierzu zählen insbesondere die Komplexität der Bedarfslagen und die Vielzahl der zur „Problembearbeitung“ einzuschaltenden Stellen. Wenn aber rechtskreisübergreifendes Case Management in der beschriebenen Weise „selektiv“ ist, stellt sich das Problem, welche Instanz (natürlich unter Einbeziehung der Adressatinnen und Adressaten) darüber entscheidet, ob rechtskreisübergreifendes Case Management indiziert ist. Grundsätzlich sind hier drei Varianten denkbar:

- Alle Ratsuchenden suchen einen Case Manager auf, der zunächst ein intensives Beratungsgespräch führt, um festzustellen, ob rechtskreisübergreifendes Case Management notwendig ist oder nicht („screening“"). In den Fällen, in denen rechtskreisübergreifendes Case Management indiziert ist, übernimmt der Case Manager den Fall selbst oder vermittelt direkt an eine Kollegin bzw. einen Kollegen.

- Alle Ratsuchenden werden in einer Service-/Anlaufstelle beraten. Sollte sich im Zuge der Beratung die Notwendigkeit eines speziellen rechtskreisübergreifenden Case Managements zeigen, wird an eine hierfür geeignete Person (Case Manager) verwiesen.

- Die Ratsuchenden werden an unterschiedlichen Stellen beraten (Ausländerbehörde, Soziale Arbeit in Unterkünften etc.). Sollte sich im Zu-

49 Näheres ist Kapitel 4 zu entnehmen. 
ge der Beratung die Notwendigkeit eines speziellen rechtskreisübergreifenden Case Managements zeigen, wird an eine hierfür geeignete Person (Case Manager) verwiesen.

Bei der erstgenannten Variante ergibt sich die Notwendigkeit eines vergleichsweise höheren Personaleinsatzes von Case Managern, da diese zusätzlich zum rechtskreisübergreifenden Case Management Beratungen durchführen. Bei den beiden anderen Varianten stellt sich hingegen die Notwendigkeit einer ausreichenden Kompetenz der Erstberatenden, die Indikation für rechtskreisübergreifendes Case Management festzustellen. Dazu ist es notwendig, dass sich Erstberatende und Case Manager fortlaufend über die Kriterien für rechtskreisübergreifendes Case Management verständigen, um Reibungsverluste zu minimieren.

\subsection{Rechtskreisübergreifendes Case Management ist häufig nicht das einzige Angebot}

Im Feld der Migrationspolitik haben sich seit Längerem zwei Angebote etabliert, die über die jeweilig geltenden Förderrichtlinien angehalten sind, Case Management für Zugewanderte anzubieten (Jugendmigrationsdienst, Migrationsberatung für erwachsene Zugewanderte). Diese Angebote sind nicht flächendeckend und (zumindest zurzeit) quantitativ nicht ausreichend, dennoch stellen sie dort, wo sie existieren, wichtige Kooperationspartnerinnen und -partner für das kommunale rechtskreisübergreifende Case Management dar. Existiert aber mehr als ein Anbieter, stellt sich die Notwendigkeit einer systematischen Kooperation, da alle Anbieter auf das lokale Netzwerk zurückgreifen sollen, ohne dass Konkurrenzen zu suboptimalen Ergebnissen führen.

Dies bedeutet, dass ein gemeinsames Konzept entwickelt werden muss, das folgende Eckpunkte enthält:

- Prozessstandards für rechtskreisübergreifendes Case Management (angelehnt an die Standards und Richtlinien der DGCC).

- Verabredete Verfahren zur Zuständigkeit für Ratsuchende.

- Festlegung gemeinsamer Dokumentationssysteme/Statistiken. 


\subsection{Integrationsmanagement ist rechtskreisübergreifendes Case Management}

Die eben beschriebenen Prozesse gelten für adressierte Personen, die Leistungen nach dem AsylbLG beziehen, also Personen mit Aufenthaltsgestattung oder einer Duldung nach $\$ 60$ a Aufenthaltsgesetz. Personen mit Aufenthaltserlaubnis haben dem Grunde nach Ansprüche auf Leistungen des SGB II. Damit werden nach $\$ 14$ SGB II „persönliche Ansprechpartner“ zuständig, die u. a. auch „Fallmanagement" durchführen sollen. Damit erfolgt ein „Rechtskreiswechsel“ (vom SGB III zum SGB II), der nicht nur die Rechtsgrundlage für Leistungen zur Arbeitsmarktintegration betrifft, sondern auch die Zuständigkeit für Beratungsleistungen (rechtskreisübergreifendes Case Management bzw. Fallmanagement). Bei der Bearbeitung dieser, durch die Gesetzeslage geschaffene, Schnittstelle zwischen „Rechtskreisen" sind folgende Varianten denkbar:

- Wenn das Fallmanagement im SGB II oder ggf. zusätzliche Angebote des lokalen Jobcenters nicht nur Belange der Arbeitsmarktintegration, sondern auch weitergehende Bedarfslagen abdecken, kann eine „warme Übergabe" erfolgen, um eine Zielperson vom Einwanderungs- in das Fallmanagement zu geleiten. Systematisch abgesichert wird dies über eine entsprechende Kooperationsvereinbarung zwischen Stadt und Jobcenter.

- Bei einem zugelassenen kommunalen Träger („Optionskommune“) wurde bereits 2005 ein Vertrag zwischen Stadt und Jobcenter (damals als ARGE organisiert) geschlossen, um sicherzustellen, dass Sprachintegrationskurse bis zum Ende besucht werden, was nur durch ein umfassendes Case Management möglich war. Dies ist als Ergänzung zum Fallmanagement des Jobcenters zu sehen, wobei die Stadt als dessen Aufragnehmerin agiert. Die berufliche Anerkennung des Sprachkurses ist geklärt, die Kosten werden vom Jobcenter erstattet, da es sich um eine Leistung zur Arbeitsmarktintegration handelt.

- Wenn das Jobcenter sich überwiegend für die Arbeitsvermittlung zuständig sieht, kann das rechtskreisübergreifende Case Management die Bearbeitung der darüber hinausgehenden Bedarfslagen übernehmen. Das kann als „freiwillige Leistung“ geschehen oder aber im Rahmen des $\mathbb{1} 16$ a SGB II als „psychosoziale Betreuung“. Im letzteren Fall gibt es eine klare Rechtsgrundlage für die Leistung und sie ist auch als Eingliederungsleistung im Rahmen des SGB II zu bilanzieren. In beiden Fällen bleibt aber das Fallmanagement nach $\$ 16$ a SGB II qua Gesetz zuständig und damit federführend. 
Die hier verhandelten Problemstellungen zeigen eine Besonderheit des rechtskreisübergreifenden Case Managements: Sowohl auf der Strukturebene als auch auf der Ebene des Einzelfalls sind Verhandlungen mit Kooperationspartnerinnen und -partnern erforderlich, die auf der gleichen Grundlage, nämlich der des Handlungskonzepts Case Management agieren - zum einen auf der gleichen Ebene, zum anderen (SGB II) sogar als Auftragsgebende. Es geht deshalb nicht um „Linking“ als Prozessschritt des Case Managements, sondern beinhaltet gegenüber der „klassischen“ Case Management-Konstellation neue Aspekte und neue Rollen. Dies gilt es bei der Festlegung von Strukturen und Prozessen ebenso zu berücksichtigen wie in der Weiterbildung.

\subsubsection{Konzeptionelle Vorentscheidungen zum Case Management}

Die in Abschnitt 5.3.2 diskutierten Kontextbedingungen bilden den Rahmen für die Konzipierung eines bereichsspezifischen Case Managements, in unserem Falle des „rechtskreisübergreifenden Case Managements“. In diesem Abschnitt geht es darum, welche konzeptionellen Vorentscheidungen vor diesem Hintergrund getroffen werden müssen, um den Prozess des Case Managements real in Gang setzen zu können.

Grundsätzlich müssen im Rahmen des „Designs“ eines Case Management-Prozesses Ressourcen und notwendige Bedingungen geklärt werden. Dabei können Ressourcen und Bedingungen auf zweierlei Weise mit konzeptionellen Überlegungen vermittelt sein: Einerseits gesetzt als ,gegebene Voraussetzungen" in Form vorhandenen Personals, vorhandener Budgets etc. und mit dieser Setzung die Möglichkeiten des Designs begrenzen; die zentrale Frage lautet dann: Wieviel Case Management ist möglich? Andererseits kann im umgekehrten Fall der Ausgangspunkt ein Konzept sein, das aus der Kontextanalyse definiert, wie ein sinnvolles Case Management aussehen könnte, und damit das wünschbare Leistungsangebot umreißt, um von hier aus notwendige Ressourcen und Bedingungen festzulegen. Die zentrale Frage lautet dann entsprechend: Was muss getan werden, um Case Management in fachlich angemessener Weise zu realisieren? Es hängt von den konkreten Gegebenheiten „vor Ort“ ab, welche der beiden Varianten zum Tragen kommt. Allerdings beinhaltet die Wahl der erstgenannten Frage unter Umständen, dass Case Management gar nicht oder nur als Alibi-Veranstaltung möglich ist. Unabhängig von der Frage, ob das Budget das Konzept begrenzt, oder das Konzept das Budget festlegt, sollte ein Konzept des Case Managements in der Arbeit mit Eingewanderten Überle- 
gungen zum Auftrag, zum spezifischen Zuschnitt der Interaktionsbeziehung, zur funktionalen Ausgestaltung, zu Qualifikation und Kompetenz des Personals sowie zur Steuerung des Angebots umfassen.

\subsubsection{Der Auftrag}

Bei der Konzipierung des Leistungsangebots gilt es zunächst, den grundlegenden Auftrag des Case Managements zu prüfen. Dieser resultiert aus der Problemwahrnehmung durch die zentralen Akteure (z. B. als Folge einer Bedarfs- und Bestandsanalyse) und den Konstellationen im politischen wie organisatorischen Kontext, die die Umsetzung von Konzepten beeinflussen. Dies zeigt sich im Falle des rechtskreisübergreifenden Case Managements bereits bei der Definition der Zielgruppe: Geht es um Personen mit „guter Bleibeperspektive“ unter Ausgrenzung der anderen oder um ein Angebot für alle, die in Deutschland angekommen sind? Dies bedeutet nicht zuletzt, ob rechtskreisübergreifendes Case Management die Selektionen nachvollzieht und verstärkt, die migrationspolitisch von Seiten des Staates gesetzt werden, oder ob sich rechtskreisübergreifendes Case Management an elementaren Menschenrechten ausrichtet und auch dort Dienstleistungen gewährt und organisiert, wo Aufenthaltsrechte prekär und materielle Leistungen eingeschränkt sind.

Der Auftrag kann darin bestehen

- die Leistungsgewährung zu optimieren, d. h. effektiver und effizienter zu gestalten,

- die Bedürfnisse der Adressatinnen und Adressaten zu entdecken und durch Vernetzung professioneller Angebote zu befriedigen - in Abhängigkeit oder unabhängig vom aufenthaltsrechtlichen Status (s. o.),

- die Adressatin und Adressaten dabei zu unterstützen, ihre bzw. seine Bedürfnisse zu artikulieren und sie bzw. ihn in die Lage zu versetzen, diese selbst zu befriedigen - in Abhängigkeit oder unabhängig vom aufenthaltsrechtlichen Status (s. o.).

Hier zeigt sich erneut die Differenz zwischen „consumer-driven“ und „system-driven" Case Management (vgl. Abschnitt 5.3.1). Häufig ist der Auftrag jedoch nicht klar definiert, deshalb sollte stets versucht werden, die fallübergreifenden strategischen Ziele festzulegen bzw. zu rekonstruieren, unter denen Case Management eingeführt wird (Frage: Was soll mittelfristig erreicht werden?). So können aus der Analyse des Hilfebedarfs und des Hilfsangebots Veränderungsoptionen und hieraus strategische Ziele abgeleitet werden, welche die Gegebenheiten der örtlichen Situation widerspie- 
geln. Die kommunale Diskussion über „Integration“ einer- und die Gewährleistung von Teilhabechancen und -kompetenzen andererseits wird den Stellenwert eines rechtskreisübergreifenden Case Managements und die Ressourcen für ein solches maßgeblich bestimmen. Hierher gehören auch Entscheidungen über die Zielgruppen (vgl. Abschnitt 5.3.2.1. Die strategischen Ziele legen den strukturellen Rahmen fest, in dem die konkrete Arbeit geleistet wird. Je nach Zielsetzung werden bestimmte Entscheidungen über Ressourcen und Bedingungen zu fällen sein, z. B. dürfte je nach Auftrag der Phase des Assessments besondere Aufmerksamkeit zu widmen sein, allerdings mit unterschiedlicher Ausrichtung: Kommt es beim Auftrag, Bedürfnisse zu entdecken, schwerpunktmäßig darauf an, eine teilweise tiefgestaffelte Diagnose vorzunehmen, so legt der Auftrag, Adressatinnen und Adressatenbei der Artikulation von Bedürfnissen zu helfen, eher nahe, das Assessment motivierend auszurichten. Beim Auftrag einer effektiveren Ausgestaltung der Leistungsgewährung hängt es vom Ziel ab, ob Assessment- und Diagnoseprozesse eine vertiefte Rolle spielen.

Am Beispiel der Einführung von Case Management in die deutsche Arbeitsmarktpolitik lässt sich beobachten, welche Auswirkungen ungeklärte Ambivalenzen gegenüber den Bedarfen der Adressatinnen und Adressaten haben. Die meisten Konzepte sprechen von einer „passgenauen“, d. h. auf die individuelle Ausgangslage zugeschnittenen, Hilfeleistung. Dieser individuelle Zuschnitt legitimiert dann auch Kontraktgestaltungen mit gegenseitigen Verpflichtungen („Fördern und Fordern“) und Sanktionen bei Vertragsbruch. Gleichzeitig soll die Hilfe Einsparungen beim ALG II erzielen, was sie von vornherein unter das Diktat einer Kosten-Nutzen-Rechnung stellt. Gelingt die Auflösung der Zwiespältigkeit nicht, muss beobachtet werden, welche der genannten Faktoren Vorrang genießen und ob eine Vernachlässigung anderer Faktoren ohne Folgen bleibt.

Die Auftragsklärung bzw. Festlegung strategischer Ziele kann nicht als einmaliger Akt, sondern muss als ein (zentrales) Element eines fortlaufenden Prozesses organisiert werden, denn die Ergebnisse der Fallarbeit - insbesondere das Feststellen von Angebotslücken - sollten in die Bedarfsanalyse einfließen und damit auf strategische Zielsetzungen rückwirken. Für alle Zielgruppen ist der Einsatz von Case Management ähnlich zu diskutieren: Welche Zielvorgaben werden unabhängig von der individuellen Bedarfslage gemacht und welchen Spielraum haben Case Manager im Umgang mit dem organisationalen Auftrag? 


\subsubsection{Der Zuschnitt der Interaktionsbeziehung ${ }^{50}$}

Zunächst ist zu klären, ob die Intervention durch das Case Management „direkt" oder „indirekt" erfolgen soll, denn dies wiederum hat Folgen für die Intensität des Arbeitseinsatzes und die erforderlichen Kompetenzen der Case Manager: „Direkte Intervention ist die unmittelbare Arbeit des Case Managers mit den Adressaten, mit dem Ziel, deren Fähigkeiten und Möglichkeiten bei der Sorge um sich selbst, bei der Inanspruchnahme von Dienstleistungen, der Selbstkontrolle und bei der Erforschung von Hindernissen, die der Befriedigung ihrer Bedürfnisse entgegenstehen, zu verbessern“ (Moxley 1997: S. 56). Case Management hat hier eine im Kern pädagogische Ausrichtung und ist - trotz aller Notwendigkeit, Angebote zu koordinieren (s. u.) - stark einzelfallorientiert. Dagegen akzentuiert indirekte Intervention stärker den Kontakt zu Leistungsangeboten Dritter und setzt auf deren Steuerung im Dienste der mit den Adressatinnen und Adressaten ausgehandelten Ziele. „Indirekte Intervention fokussiert die Aufmerksamkeit des Case Managers auf die Interventionen in Unterstützungssysteme, was auch die Arbeit mit Dienstleistungssystemen und Familien beinhalten kann. Dies dient dem Ziel, die im Einzelfall benötigten Dienstleistungen zu kombinieren und die Leistungsadressaten sozial zu unterstützen sowie Hindernisse zu identifizieren und zu beseitigen, um dadurch die Bedürfnisse komplett abzudecken" (ebd.). Diese Unterscheidung weist auf eine Unschärfe im Zuschnitt des Case Managements hin, die in der Praxis zu Schwierigkeiten führen kann: Vom Grundverständnis her „vermittelt“ das Case Management Leistungen (d. h. „sekundäre Dienstleistungen“) und erbringt sie nicht selbst, hier sind die Case Manager in der Funktion von „Brokern“. Gleichzeitig setzt ein Case Management-Prozess den Aufbau einer Vertrauensbasis, eines „Arbeitsbündnisses“ voraus. Und hierzu wird es in vielen Fällen, insbesondere dann, wenn die Case Manager sich in einer „anwaltlichen“ Funktion befinden, notwendig sein, eine intensivere Beratungsbeziehung aufzubauen und über längere Zeit Kontakt zu halten.

Letztlich ist eine Abgrenzung von Case Management und traditioneller Einzelfallhilfe in der Praxis (nicht in der Konzeption!) schwierig und manchmal nur von Fall zu Fall möglich. Allerdings sollte man sich vor zwei konzeptionellen Fehlern hüten, die folgenreich sein können:

- Der Verwechslung von Case Management und Case Work, d. h. dem planenden und steuernden Management eines Falls und der auf den

50 Ausführlich hierzu Abschnitt 5.5. 
Einzelfall bezogenen Sozialen Arbeit. Diese Verwechslung kann dazu führen, dass die sozialpädagogischen Anteile von Maßnahmen für die Zielgruppe mit dem Hinweis auf die Existenz von Case Management reduziert oder gar gestrichen werden. Demgegenüber ist darauf zu insistieren, dass die konzeptionelle Aufgabe von Case Management darin besteht, Probleme der adressierten Person zu identifizieren (was nur gelingt, wenn eine Beratungsbeziehung aufgebaut wird) und dann zu deren Bearbeitung an einen Fachdienst zu verweisen.

- Dem Irrglauben, die Identifikation von Problemen sei vergleichsweise einfach, so dass die Aufgabe von Case Management in der Verweisung an Fachdienste liege. Hier ist daran zu erinnern, dass sich die Notwendigkeit von Case Management aus der Komplexität von Problemlagen ergibt, die häufig so miteinander verschränkt sind, dass ein einzelnes Angebot oder ein einzelner Fachdienst nicht ausreicht, sondern „Dienstleistungsbündel“ geschnürt werden müssen. Um dies zu tun, ist es notwendig, nicht nur Probleme zu erkennen, sondern auch sinnvolle Bearbeitungswege zu entwerfen. Auch hier zeigt sich wieder die Notwendigkeit der Verknüpfung der Steuerung des Einzelfalls mit der Steuerung der Angebotsebene.

\subsubsection{Funktionale Ausgestaltung}

Von den Zielsetzungen des Case Managements und der grundsätzlichen Ausrichtung wird auch die Gewichtung der drei möglichen Funktionen des Case Managements für ihre Zielgruppe abhängen. Jede dieser Funktionen betont bestimmte Lesarten der Grundfunktion „Vermittlung“ und verlangt spezifische Zielsetzungen und Rollenanforderungen vom Case Manager: ${ }^{51}$

- „Advocacy“, d. h. die anwaltliche Funktion. Sie „zielt auf Menschen, die angesichts konfliktträchtiger Lebenssituationen (zum Beispiel Armut, mangelnde Bildung, Behinderung) oder unvorhergesehener biographischer Lebenskrisen (zum Beispiel Unfall, Arbeitslosigkeit, schwere Erkrankung) nicht in der Lage sind, ihre persönlichen Interessen aufgrund von kurz-, mittel- oder langfristiger individueller Hilfsbedürftigkeit und/oder gesellschaftlicher Machtlosigkeit geltend zu machen“" (Ewers 2000b: S. 63). Die Handlungslogik, mit der diese Funktion reali-

51 Ewers, an dessen Darstellung wir hier anschließen, spricht von drei Funktionen, Wendt dagegen von „Berufsrollen“ (vgl. Wendt 1997: S. 145ff.). 
siert wird, besteht darin, den „Fall“ konsequent aus der Perspektive der Adressatin bzw. des Adressaten zu sehen und die notwendigen Hilfen an den individuellen Bedürfnissen der Adressatin bzw. des Adressaten auszurichten. Hierzu gehört auch, Versorgungslücken zu entdecken und durch die Entwicklung geeigneter Angebote zu schließen: „Im Sinne einer Bottom-up-Strategie will Case Management zu einem patientenorientierten und bedarfsgerechten Ausbau des Sozial- und Gesundheitssystems und zu einer effizienteren Lösung vorhandener Versorgungsprobleme beitragen“ (ebd.: S. 65). Zur Logik der "anwaltlichen“ Funktion gehört es dabei, aus Abhängigkeiten und Machtlosigkeit herauszuführen und diese nicht etwa zu verstärken. Dies bedeutet, im Sinne des „Empowerments“ individuelle Bewältigungsstrategien zu entdecken und/oder zu entwickeln. Der Case Manager nimmt hier die Rolle eines „Coachs" ein, „indem er zur Reflexion über das Handeln des Klienten und seines sozialen Netzwerkes anregt. Reflexion des eigenen Handelns heißt, darüber nachzudenken, welche alternativen Handlungsweisen in einer gegebenen Situation möglich sind, um so dem Ziel, Veränderungen anzubahnen, gerecht werden zu können" (Oostrik/Steenbergen 2000: S. 259). Die anwaltliche Logik besteht somit weder darin, all das zu tun, was Adressatinnen und Adressaten wünschen, noch darin, ihnen „bedarfsgerechte“ Lösungen überzustülpen, sondern entfaltet sich in der Aufgabe, gemeinsam mit ihnen situationsangemessene Bewältigungsstrategien zu entwerfen und durch entweder bereits vorhandene oder noch zu entwickelnde Hilfsangebote zu verstärken. Dies setzt auf Seiten der Case Manager voraus, dass sie einerseits in der Lage sind, eine kontinuierliche, auf das persönliche Wachstum der adressierten Personen orientierte professionelle Beziehung zu diesen einzugehen und andererseits nicht nur die professionellen und die jeweiligen Netzwerke der Adressatinnen und Adressaten gut kennen, sondern auch kompetent zu nutzen wissen.

- „Broker“, d. h. die vermittelnde Funktion. Die Handlungslogik der „anwaltlichen Funktion“ ist somit stark durch adressierte Personen und Netzwerkorientierung gekennzeichnet und betont den Teil des Case Managements, der in der deutschen Debatte mit „Passgenauigkeit" bezeichnet wird - „Broker“, d. h. die vermittelnde Funktion. Die Handlungslogik besteht hier darin, einen möglichst umfassenden Überblick über das Hilfsangebot zu gewinnen und Verbindungen zwischen individuellem Bedürfnis und diesem Angebot herzustellen, um so ein „individuelles Versorgungspaket“ zu schnüren (vgl. Ewers 2000b: S. 68) und dessen Umsetzung in die Wege zu leiten. Die Realisierung dieser 
Handlungslogik erfordert eine "neutrale“ Position, denn es gilt zwischen den beiden Polen „Adressatin und Adressat" und „Versorgungssystem" zu vermitteln. Im Zentrum des Handelns steht auch weniger die Adressatin bzw. der Adressat und deren Problemlage, als vielmehr der Prozess der Leistungserbringung, d. h. die Koordination und Überwachung („Monitoring“ und „Controlling“), die Handlungslogik ist im Kern somit „Steuerungslogik“.

- „Gate-Keeper“, d. h. selektierende Funktion. Da der Zugang zu den Ressourcen des Versorgungssystems durch rechtliche und/oder administrative Regeln begrenzt wird, ist eine wesentliche Teilfunktion des Case Managements die der "Zugangssteuerung“, d. h. der Selektion, welche Personen in Prozesse des Case Managements aufgenommen werden und welche nicht, und welche Hilfen und Dienstleistungen kostenwirksam eingesetzt werden. Diese Funktion bringt die dem Einzelfall übergeordneten gesellschaftlichen Belange zum Ausdruck. Gerade hierin hat ein "Gate Keeper" auch die Aufgabe, auf die Effektivität des Hilfeprozesses und die Effizienz der einzelnen Angebote zu achten. Die Handlungslogik ist dabei weniger durch „Aushandeln“ (wie in der vermittelnden und anwaltlichen Funktion) als vielmehr durch „Kalkulieren“ und „Kontrollieren“ geprägt, folgt also einer betriebswirtschaftlichen Logik: Es geht darum, darauf zu achten, dass das Versorgungspaket nicht nur an die individuelle Situation angepasst (d. h. „passgenau“) ist, sondern effektiv und effizient umgesetzt wird. „Der Case Manager soll (...) die Gewähr dafür bieten, dass sowohl bei den Leistungserbringern als auch bei den Nutzern ein Bewusstsein für die wirtschaftlichen Folgen des Versorgungsgeschehens entsteht und der Rückgriff auf vorhandene Ressourcen im Sinne der sparsamen Mittelverwendung beeinflusst wird“ (ebd.: S. 71). Wissert (2001) definiert darüber hinaus die Rolle des Social Supporters, die über die anwaltliche Funktion hinausgeht. Zunächst steht hier im Fokus, die Adressatinnen und Adressaten in sehr belasteten Lebenssituationen zu befähigen und/oder zu motivieren, Unterstützungsangebote zu nutzen.

Keine dieser Funktionen ist in der hier dargestellten idealtypischen Form für sich allein realisiert, vielmehr werden die unterschiedlichen Funktionen in diversen Case Management-Konzepten in vielfältiger Weise kombiniert. Allerdings korrespondieren diese Funktionen mit der System- und Adressatenorientierung eines Case Management-Konzepts und somit wirken sich Schwerpunktsetzungen an den beiden Polen der anwaltlichen bzw. selektierenden Funktion stark auf die Gesamtausrichtung eines Konzepts aus. 


\subsubsection{Qualifikation und Kompetenz des Personals}

Vom grundlegenden Auftrag, der Ausrichtung und den jeweils bevorzugten Funktionen des Case Managements wird abhängen, welche Anforderungen an die Kompetenz des Personals zu stellen sind, d. h. über welche formalen Qualifikationen und Fähigkeiten die Case Manager jeweils verfügen müssen. Komplexere Case Management-Prozesse können sogar Konzepte des Case Managements im Team erfordern (vgl. Moxley 1997: S. 54). Für die Koordination der das Case Management unterstützende Netzwerke sind weitere Kompetenzen notwendig, insbesondere für die Zusammenarbeit mit kommunaler Sozialplanung. Festzulegen ist auch, welche Personengruppen Case Management durchführen sollen und ob dies ausschließlich von ihrer Grundqualifikation sozialarbeiterisches oder -pädagogisches Personal sein muss. Dahinter verbirgt sich neben der Diskussion um De-Professionalisierung der Streit zwischen Berufsgruppen (z. B. Verwaltungsfachkräfte gegen Sozialarbeiterinnen und -arbeiter).

Um Missverständnissen vorzubeugen: Unabhängig von der grundlegenden Ausrichtung und Ausgestaltung fußt Case Management trotz der Betonung der „Vermittlungsfunktion“ auf einem Arbeitsbündnis zwischen Case Management und Adressatinnen und Adressaten (vgl. Abschnitt 5.5). Auch wenn es „nur“ um die Vermittlung von Leistungen geht, die andere erbringen sollen, ist der Aufbau eines Vertrauensverhältnisses wichtig, um adressierte Personen zur Mitwirkung am Case Management zu motivieren. Dies gelingt nur über den Aufbau eines tragfähigen Arbeitsbündnisses. Lamb/Stempel (2000) beschreiben die Rolle der Case Manager hier zutreffend als eine des „Insider-Experten“, die oder der auf der einen Seite das Leistungsangebot kennt, aber auch ein Gefühl entwickelt für die Problemlage der Adressatin bzw. des Adressaten, ihre oder seine Selbstwahrnehmung sowie Fähigkeiten und Schwächen und diese bei der Vermittlung von Leistungen in Rechnung stellt. Case Management empfiehlt sich gerade für Zielgruppen, deren Bewältigungsressourcen oft für Anforderungen von an sich passenden Maßnahmen nicht ausreichen.

Um diesen Anforderungen gerecht zu werden, sind für Case Manager neben umfassenden Kenntnissen von Case Management, einem Überblick über die lokale Angebotsstruktur, vorhandene Netzwerke sowie Wissen über relevante Rechtsgebiete immer auch Kompetenzen in Beratung notwendig - und zwar unabhängig von der ursprünglichen Qualifikation. 


\subsubsection{Angebotssteuerung}

Auf der konzeptionellen Ebene ist auch zu klären, welche Angebote fallübergreifend koordiniert werden sollen, dabei geht es um Fragen der Angebotsentwicklung, -koordination und -steuerung. Das Formulieren strategischer Ziele macht nämlich nur dann Sinn, wenn diese auch in konkrete Angebote und Leistungen umgesetzt werden und dann die Zielerreichung überprüft wird. Die Planung von Angeboten wird sich vielerorts so darstellen, dass eine vielgliedrige Trägerlandschaft koordiniert werden muss, denn Beratungs- und Qualifizierungsangebote werden von unterschiedlichen Trägern vorgehalten. Die Koordination der Anbieter und Träger bedeutet im Kontext integrierter Hilfe die gemeinsame Planung eines künftigen Leistungsangebots - mit der zentralen Ausrichtung am prognostizierten Bedarf und nicht an Maßnahmeplätzen, die „besetzt werden müssen“. Hier muss vielerorts ein grundsätzlicher strategischer Wechsel vollzogen werden, der allen Beteiligten ein Umdenken abverlangt.

Der Gedanke des einzelfallbezogenen Case Managements basiert auf der Vorstellung, dass die notwendigen Leistungsangebote vorhanden sind oder kurzfristig bereitgestellt werden können (vgl. auch Moxley 1997: S. 69). Zugespitzt betrachtet bedeutet dies, dass der Fortschritt des Case Managements von einer Weiterentwicklung des professionellen Angebots wie auch insgesamt des „Community support system“, also des Systems formeller und informeller Hilfen, abhängt. Dies muss klar gesehen werden, darf aber nicht dazu führen, dass die einzelnen Case Manager mit Aufgaben überlastet werden, die ihre Steuerungskompetenz überfordern. Moxley formuliert dies so: „Das Gewicht der Gemeinschaftsunterstützung kann nicht ganz oder nicht einmal vorwiegend auf die Schultern des Case Managements geladen werden“ (ebd.: S. 70). Dies gilt in besonderem Maße für das Case Management in der Arbeit mit Zielgruppen, deren Teilhabechancen von der Gesellschaft unabhängig von ihren Teilhabekompetenzen eingeschränkt werden. Case Management kann strukturelle Defizite nicht mit der Arbeit an Einzelfällen bewältigen. Ein typisches Beispiel ist hier Vermittlung von Kenntnissen zum Thema Wohnen an Geflüchtete („Wohnfähigkeit“), die den Mangel an (bezahlbarem) Wohnraum nicht kompensiert. Es würde das Instrument Case Management komplett überfordern, wenn von ihm die Lösung von strukturell verursachten Problemstellungen verlangt bzw. sein Erfolg hieran gemessen würde. 


\subsubsection{Zusammenfassung}

Für die Erstellung eines Konzepts für Case Management sind folgende Fragestellungen hilfreich:

1. Welche Auswirkungen auf Schwerpunktsetzungen im Case Management hat der spezifische Auftrag? Welche der Teilfunktionen hat dabei besonderes Gewicht? Ist diese Schwerpunktsetzung mit der Problemund Zielgruppenanalyse vereinbar?

2. Welche fallübergreifenden Ziele hat das Case Management? Wie wirken sich diese Ziele auf die Gestaltung der Arbeit im Einzelfall aus?

3. Welche grundlegende inhaltliche Ausrichtung sollte das Case Management haben (Einzelfall und Sozialraum)? Wie sieht die Einbindung in sozialräumliche Strategien aus? Ist diese Ausrichtung mit dem politischen Auftrag kompatibel?

4. Welche Organisationsprinzipien sollten der Ausgestaltung des Case Managements zu Grunde gelegt werden (Strukturerweiterung, flächendeckende Änderung der Arbeitsweise)?

5. Welche Steuerungskompetenzen benötigen die Case Manager?

6. Welches Personal mit welchen formalen Qualifikationen und Fähigkeiten wird benötigt (Verwaltungsfachkräfte, Sozialarbeiterinnen und Sozialarbeiter, multiprofessionelle Teams)?

7. Welche Formen der Vernetzung mit den einzelnen Leistungsanbietern sollten gewählt werden (Fallkonferenzen, marktanaloge Einbindung, Planungskonferenzen)?

8. Wie sieht der Leistungsumfang aus, auf dessen Vermittlung sich das Case Management erstreckt? Reicht dieser aus, um die Ziele zu erreichen?

9. Wie sieht die grundsätzliche Zeitschiene aus (kontinuierliche Begleitung eines Falls, Begleitung nur für Zeitabschnitte)?

10. Wie ist die Koordination von Netzwerken organisiert? Wie funktioniert der systematische Austausch zwischen Case Management, Netzwerkkoordination, Angebotssteuerung und Sozialplanung?

\subsection{Ein komplexes Konzept von Case Management}

(Rechtskreisübergreifendes) Case Management ist in erheblichem Maße von der Vermittlung zwischen einzelfallunspezifischen, einzelfallübergreifenden und einzelfallspezifischen Elementen geprägt. Um die damit einhergehende Komplexität der Aufgaben bewältigen zu können, ist es not- 
wendig, die einzelnen Prozesse, also die einzelfallunspezifischen und -übergreifenden Tätigkeiten sowie die fallbezogenen Interaktionen, klar zu strukturieren. Ein wichtiger Erfolgsfaktor für das Case Management ist somit auch die Definition von einzelfallspezifischen Verfahrensabläufen und Schlüsselinterventionen sowie die Festlegung entsprechender Qualitätsstandards. Die einzelnen Prozessschritte müssen konkretisiert und beschrieben werden (vgl. Schaubild 11).

Schaubild 11: Der Regelkreis des Case Managements

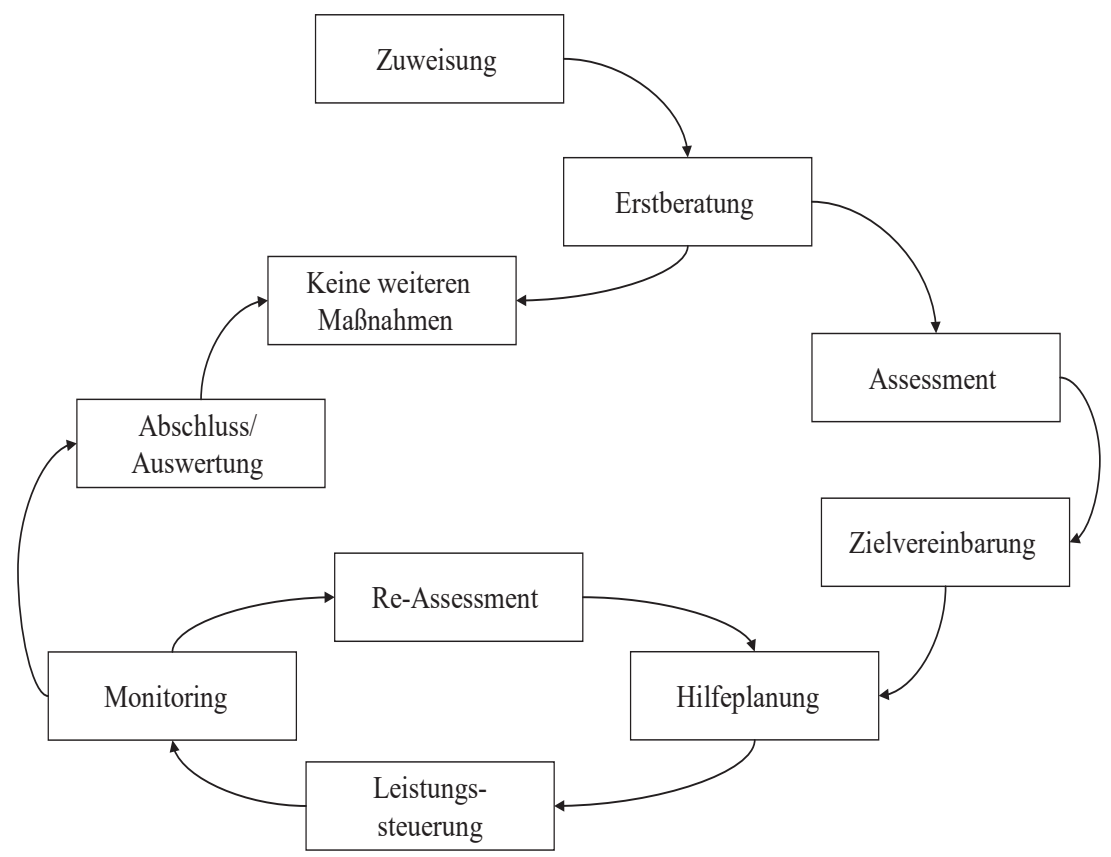

(eigene Darstellung)

Die Konzipierung von Case Management als einzelfallbezogener Hilfe stützt sich auf Erkenntnisse, die unabhängig von Einzelfällen gewonnen wurden (Bedarfs- und Bestandsanalyse). Auch der politische und organisationale Kontext von Case Management hängt nicht von Einzelfällen ab, prägt aber entscheidend die Zielsetzungen und damit die Ausgangslage. Wenn mit Case Management der Anspruch verknüpft ist, nicht nur Leistungsangebote zu koordinieren, sondern dies auch in einer Weise zu tun, die den individuellen Bedarf der jeweiligen Adressatin oder des jeweiligen 
Adressaten einbezieht, dann muss es über die Steuerung des vorhandenen Angebots hinausgehen, über eine Analyse nicht gedeckter Bedarfe Angebotslücken feststellen und sich dafür engagieren, diese zu schließen. Geschieht dies nicht, wird Case Management reduziert auf die Anpassung individueller Bedarfslagen an ein vorhandenes Angebot, das Ziel der „passgenauen Hilfe" kann in der Regel so nicht erreicht werden.

Case Management muss daher, soll es tatsächlich den Anspruch „passgenauer Hilfe“ einlösen, neben der einzelfallbezogenen Arbeit einzelfallübergreifende Aktivitäten entfalten und sich systematisch an der Steuerung des Gesamtangebots beteiligen. Dies bedeutet, dass die Einführung von Case Management beteiligte Organisationen und Akteure an drei Stellen herausfordert:

- Ansätze der Bedarfsanalyse, Sozialberichterstattung und Sozialplanung müssen verstärkt werden, um den Boden für effektives, einzelfallübergreifendes und -unabhängiges Arbeiten zu bereiten.

- Instrumente der Koordinierung und Angebotssteuerung müssen ausgebaut und neu entwickelt werden - hierzu gehört auch ein funktionsgerechtes Controlling.

- Einzelfall- und Angebotssteuerung müssen systematisch miteinander verknüpft werden - dies stärkt die Kompetenz der Case Manager, denn sie entscheiden durch ihre Arbeit unmittelbar über die künftige Ausgestaltung des Angebots mit.

In der folgenden Darstellung der einzelnen Prozessschritte wird sowohl die einzelfallbezogene Ebene behandelt als auch die der Organisation, die mit Case Management arbeitet. Auf den Einzelfall bezogene Handlungsempfehlungen werden durch Gestaltungsvarianten institutioneller und organisationaler Lösungen ergänzt, um ein komplexes Case Management zu diskutieren, das Fall- und Angebotssteuerung verbindet.

\subsubsection{Erstberatung}

„Beratung“ ist Bestandteil mehrerer Prozessschritte des Case Managements. So hat sie ihren Ort in der Erstberatung, aber unter Umständen auch im Rahmen des Assessments, der Zielvereinbarung und der Hilfeplanung. Mit „Erstberatung“ verknüpfen sich aber bestimmte Aufgaben, die es erlauben, diesen Prozessschritt besonders hervorzuheben.

Die Erstberatung als Prozessschritt eines Case Managements erfüllt insgesamt komplexe Aufgaben. Zunächst geht es für Case Manager darum, einen ersten Eindruck von der Problemlage der Ratsuchenden zu bekom- 
men und deren Motivation zu klären sowie ggf. aufzubauen. Auf der Basis dieser Informationen kann dann eine Entscheidung über das weitere Vorgehen getroffen werden. Hierzu gehört die Entscheidung, ob angesichts von Problemlage und Motivation überhaupt der aufwendige Weg eines Case Managements beschritten werden soll, denn nicht in allen Fällen ist dies nötig oder möglich (z. B., wenn sich adressierte Personen in akuten Krisensituationen befinden). Gegenstand der Erstberatung ist auch die Herstellung von Transparenz über das Verfahren, z. B. die Aufklärung über die auf die Ratsuchenden zukommenden Anforderungen, die gegenseitigen Rollen, aber auch die Chancen und Möglichkeiten, die in dem Verfahren stecken. Hier sollte allerdings Realitätssinn herrschen, denn nichts demotiviert mehr als überhöhte Erwartungen, die dann enttäuscht werden. Dieser Prozessschritt dient somit u. a. der Feststellung, ob adressierte Personen in das Case Management „aufgenommen“ werden oder nicht. Case Management ist eine zeit- und personalintensive soziale Dienstleistung und von daher muss entschieden werden, wer sie erhalten soll und wer nicht. Parallel muss entschieden werden, ob es tatsächlich zu einem gemeinsamen Arbeitsprozess kommen kann und kommen soll. Nicht jede Person, für die Case Management theoretisch die geeignete Hilfe darstellt, ist bereit oder in der Lage, sich auf diesen Prozess einzulassen umgekehrt sind auch nicht alle Case Manager bereit und in der Lage, sich mit bestimmten Personen auf diese Prozesse einzulassen (vgl. ausführlich dazu Ministerium für Wirtschaft und Arbeit des Landes Nordrhein-Westfalen 2003).

Das bedeutet, dass vor dem Beginn des eigentlichen Case Managements drei Überlegungen anzustellen sind.

\subsubsection{Konzeptionelle Überlegungen zur Indikation von Case Management}

\subsection{Zielgruppe}

Für welche Zielgruppen wird Case Management angeboten und gehört die konkrete Adressatin oder der konkrete Adressat zu einer dieser Zielgruppen? Die Beantwortung dieser Frage setzt eine Regelung auf drei Ebenen voraus.

- Es müssen in der Organisation Kriterien existieren, nach denen diese Zielgruppen möglichst exakt definiert sind. 
- Es muss Regeln und Verfahren dafür geben, wie konkrete Einzelfälle unter diese Kriterien subsumiert werden können. Wenn die Kriterien eindeutig sind und die Subsumtion deshalb vergleichsweise einfach vonstattengehen kann (z. B. durch Erfragen des Alters nach Feststellung der Hilfeberechtigung), stellt dies keine besondere Arbeit dar. Anders ist es mit Konstruktionen wie „besondere soziale Schwierigkeiten“ oder „Fälle mit komplexem Hilfebedarf“. Hier muss nach Operationalisierungen gesucht werden, wenn die Entscheidung nicht alleine dem „Gefühl“ der Fachkräfte oder den zuweisenden Stellen überlassen bleiben soll.

- Es muss festgelegt werden, wer diese Regeln und Verfahren anwendet, d. h., ob es eine Zuweisung in das Case Management gibt oder ob die Fachkräfte im Case Management selbst die „Leistungstiefe" festlegen, d. h. darüber befinden, wie intensiv sie mit bestimmten Personen zusammenarbeiten.

\subsection{Mitarbeit}

Ist die Adressatin oder der Adressat bereit und in der Lage, einen unter Umständen länger andauernden Hilfeprozess, der vom Case Management organisiert wird, „durchzuhalten“?

An dieser Stelle ist die Unterscheidung zwischen „Kooperationswilligkeit“ und „Kooperationsfähigkeit“ zu treffen. (vgl. ausführlich Ministerium für Wirtschaft und Arbeit des Landes Nordrhein-Westfalen 2003) Wenn Adressatinnen und Adressaten sich der Beteiligung am Case Management widersetzen, wird dies häufig als "Verweigerung“ angesehen und als „mangelnde Mitwirkung“ sanktioniert. Abgesehen davon, dass es gute Gründe für eine solche Haltung geben kann (z. B. die Angst, intime Dinge oder für peinlich erachtete Sachverhalte offenbaren zu müssen), kann eine solche Haltung auch darin begründet sein, dass die Adressatin oder der Adressat den Vorgang und die damit gesetzten Anforderungen nicht versteht (sprachlich oder kognitiv) oder sich nicht zutraut, sich auf einen länger andauernden Prozess einzulassen. Es muss nachdenklich stimmen, dass unter den Personen, die in den USA wegen mangelnder Mitwirkung mit Sanktionen belegt wurden, überdurchschnittlich viele Personen mit psychischen oder sprachlichen Problemen, Behinderungen oder Suchterkrankungen sind (vgl. Danziger/Seefeldt 2002). Häufig korreliert das Phänomen der Verweigerungshaltung also mit den genannten zugeschriebenen Merkmalen. Dies bedeutet, dass gerade gegenüber misstrauischen 
Personen besondere Sorgfalt an den Tag zu legen ist und dass es ein elementarer Bestandteil der Erstberatung ist, Gründe für Misstrauen, Verweigerung und Kooperationsunfähigkeit herauszufinden, um eine Entscheidung über die Aufnahme in das Case Management, die Weiterführung materieller Leistungen ohne Dienstleistung oder Sanktionen zu treffen. Das gilt auch für Personen, deren Motivation für Veränderungsprozesse in ihrem Leben unklar oder ambivalent ist. In diesem Fall ist die Klärung der Motivation und Unterstützung der adressierten Personen bei der Bearbeitung von Ambivalenzen Teil des Case Managements. Ziel ist die Stärkung der Selbstwirksamkeitserfahrungen von Adressatinnen und Adressaten (vgl. Schmid/Schu/Vogt 2012).

Case Management als Handlungskonzept geht davon aus, dass Menschen Expertinnen und Experten für ihre Bedarfe sind. Entwicklungs- und Veränderungsprozesse bei Adressatinnen und Adressaten sollten sich deshalb nach deren Bewältigungsressourcen richten und deren Stärken und Ressourcen bzw. die ihres Umfelds in den Fokus nehmen (vgl. Ehlers/ Müller/Schuster 2017). Sprachbarrieren dürfen kein Hinderungsgrund für Case Management sein. Wenn adressierte Personen und Case Manager keine gemeinsame Sprache sprechen, kann mithilfe von Dolmetscherinnen und Dolmetscher (z. B. auch durch einen Rückgriff auf Internetangebote für simultanes Video-Dolmetschen oder ehrenamtliche Sprachmittler) ein Case Management-Prozess aufgenommen werden. Bei durch körperliche oder geistige Einschränkungen verursachten Sprachprobleme kann der mutmaßliche Wille der adressierten Personen bei Angehörigen und z. B. Pflegekräften erfragt werden, die non-verbal mit Adressatinnen und Adressaten kommunizieren.

\subsection{Kommunikation}

Sind die Adressatin oder Adressat und das Case Management bereit, diesen Prozess gemeinsam zu beginnen?

Auch wenn grundsätzlich Kooperationsbereitschaft und -fähigkeit gegeben sind, kann es sein, dass die „Chemie“ zwischen Case Management und adressierten Personen „nicht stimmt“, es zu Kommunikationsproblemen kommt und aus diesem Grund die Kooperation leidet. In solchen Fällen ist es besonders heikel, auf eine grundlegende Verweigerungshaltung zu schließen. Um den Prozess in einer anderen personalen Konstellation neu zu beginnen, sollte es für adressierte Personen möglich sein, das Case Management zu wechseln. Ebenso sollten Case Manager Adressatinnen und 
Adressaten an Mitarbeitende übergeben können. Für den Prozessschritt der Erstberatung gelten - wie auch für andere beraterische Elemente des Gesamtprozesses - die Strukturelemente von „Beratung“. Werden sie außer Kraft gesetzt, sollte nicht von „Beratung“ gesprochen werden. Je mehr Handlungsfolgen mit der Beratung verknüpft werden, je verbindlicher also letztlich diese sein soll, umso prekärer ist das Entscheidungsrecht der Adressatin bzw. des Adressaten als Strukturprinzip der Beratung. Das ist im Kern verletzt, wenn Beratung die Voraussetzung für das Einlösen von Ansprüchen der Adressatin bzw. des Adressaten darstellt (vgl. MASQT 2000). Nicht Ratsuchende, sondern die Beratenden haben dann das faktische Entscheidungsrecht inne, ob die Beratung aufgenommen oder fortgesetzt wird bzw. welche Aktivitäten daraus folgen. Die Teilnahme am Gespräch ist nur bedingt bzw. überhaupt nicht freiwillig, weil sie Voraussetzung für den Erhalt sozialstaatlicher Leistungen ist; ein Element dieser Beratung ist die Überprüfung, ob und wenn ja, welche Leistungen gewährt werden können und der Abbruch der Beratung bleibt nicht grundsätzlich - wie bei den anderen Beratungstypen - ohne negative Folgen für die Ratsuchenden, sondern kann sanktioniert werden. Es bestehen Zweifel, ob diese Interaktionsform „Beratung“ genannt werden sollte, da sie gegen ein Strukturprinzip, nämlich „Ergebnisoffenheit“, verstößt. Sie stürzt Beraterende und Ratsuchende in ein strukturelles Dilemma: Es soll ein Vertrauensverhältnis entstehen, das „Beratung“ als Reflexion personenbezogener Probleme erst ermöglicht, gleichzeitig trüben restriktive Bedingungen wie die faktisch eingeschränkte Entscheidungsfreiheit der Ratsuchenden dieses Vertrauensverhältnis. Soll dennoch eine „Beratung“ zustande kommen, die von beiden Beteiligten als solche angesehen wird, muss es dem Beratenden gelingen, das häufig taktische Verhalten der Ratsuchenden (z. B. durch gezielte Motivationsarbeit) in Ko-Produktion zu transformieren, d. h. ein wirkliches Arbeitsbündnis herzustellen. Sie oder er muss damit gegen die strukturellen Bedingungen arbeiten, die die Beratungssituation prägen und steckt somit in einem „Beratungsdilemma“. Scheitert die Beratung, gelingt auch keine Beeinflussung der Wahrnehmens- und Verhaltensweisen der Ratsuchenden. Gerade wenn auf den Ergebnissen dieser Beratung komplexe Hilfsangebote aufbauen, muss die Frage aufgeworfen werden, wie sinnvoll Case Management-Konzepte sind, die das Zustandekommen eines Arbeitsbündnisses zwischen adressierten Personen und Case Managern erschweren. 


\subsubsection{Organisatorische Überlegungen zur Indikation von Case Management}

Die Entscheidung über ein Zustandekommen von Case Management kann organisatorisch unterschiedlich geregelt sein. Zwei Grundtypen lassen sich unterscheiden:

- Adressatinnen und Adressaten werden über ein spezielles qualifiziertes Verfahren („Clearing“, „Profiling“) dem Case Management „zugewiesen" oder

- die Case Manager entscheiden selbst über die „Leistungstiefe“, d. h. darüber, mit wem sie Case Management beginnen und mit wem nicht.

Im erstgenannten Fall sind die Fachkräfte im Case Management von der Entscheidung der Auswahl ein Stück entlastet, denn diese trifft eine andere Stelle. Sie können sich dann darauf konzentrieren, mit den ausgewählten Personen einen qualifizierten Prozess zu beginnen und durchzuführen. Die Erfahrung hat gezeigt, dass dies aber nur dann reibungslos funktioniert, wenn

- es klare Kriterien über die Aufnahme in das Case Management gibt und

- diese Kriterien zwischen den Beteiligten (d. h. zuweisenden Stellen und den Fachkräften im Case Management) kommuniziert und vereinheitlicht sind.

Ist dies nicht der Fall, tritt die Gefahr auf, dass die zuweisende Stelle eigene (meist nicht offen gehandelte) Kriterien entwickelt, um Adressatinnen und Adressaten in das Case Management zu überweisen, z. B., um sich mit „schwierigen“ Personen nicht selbst auseinandersetzen zu müssen, die Mitwirkungs- oder Arbeitsbereitschaft zu testen, die eigene Fallzahl zu senken etc. (vgl. Hansbauer 1996). Treffen die Case Manager selbst die Entscheidung über die Leistungstiefe, so ist die Schnittstellenproblematik aufgehoben, die Entscheidung selbst aber stark subjektiviert. Gerade wenn die Aufnahmekriterien nicht eindeutig sind, sondern Interpretationsspielräume zulassen, besteht die Gefahr, dass - analog zur anderen Zugangsvariante subjektive Gründe und Motive über die Aufnahme in das Case Management bestimmen. So können Case Manager, die selbst über die Leistungstiefe in jedem Einzelfall entscheiden, ihre Fallzahl und insbesondere die Intensität der Arbeit eigenmächtig steuern. Das gilt auch für die Variante, dass alle Erstgespräche von Case Managern geführt werden und diese dann entscheiden, welche Personen im Case Management verbleiben und welche in die „sichernde Hilfe“ übergehen. Die Fachkräfte im Case Management steuern so ihren eigenen Arbeitsanfall und den ihrer Kollegenschaft 
in der „Regelsachbearbeitung“. Zwei Arten von „Fehlsteuerung“ sind hier denkbar:

- Es werden Personen in das Case Management aufgenommen, ohne eigentlich Fälle hierfür zu sein, weil ihre Problemlagen nicht hinreichend komplex sind. In diesem Fall vermindert sich die durchschnittliche Intensität der Fälle im Case Management - was mittelfristig entlastend wirkt.

- Die Fachkräfte verweisen Personen in die „Regelpraxis“ ohne Case Management, die eigentlich Fälle für sie wären. In diesem Fall reduzieren sie ihren „Falldruck“ und erhöhen ihn gleichzeitig in der „Regelpraxis".

In der folgenden Übersicht werden Ziele, die für deren Realisierung benötigten Ressourcen, die zu lösenden Grundprobleme, die zentralen Erfolgskriterien sowie denkbare Teilschritte bei der Realisierung der Stufe schematisch nebeneinandergestellt.

1.Stufe: Erstberatung

\begin{tabular}{|c|c|c|c|}
\hline Ziel & \begin{tabular}{|l} 
Benötigte \\
Ressourcen
\end{tabular} & Grundproblem & $\begin{array}{l}\text { Erfolgs- } \\
\text { kriterium }\end{array}$ \\
\hline $\begin{array}{l}\text { Herstellung ei- } \\
\text { nes Arbeitsbünd- } \\
\text { nisses. } \\
\text { Entscheidung, } \\
\text { ob Case Manage- } \\
\text { ment stattfinden } \\
\text { soll. }\end{array}$ & $\begin{array}{l}\text { Empathie, ver- } \\
\text { bindliches Ver- } \\
\text { halten. } \\
\text { Entscheidungs- } \\
\text { kriterien. } \\
\text { Zeit. } \\
\text { Räumliches Set- } \\
\text { ting. }\end{array}$ & $\begin{array}{l}\text { Taktisches Ver- } \\
\text { halten der Ratsu- } \\
\text { chenden. } \\
\text { "Schere im } \\
\text { Kopf“ bei den } \\
\text { Case Managern, } \\
\text { d. h., es werden } \\
\text { nur Bedarfe gese- } \\
\text { hen, die auf vor- } \\
\text { handene Maß- } \\
\text { nahmen passen. }\end{array}$ & $\begin{array}{l}\text { Motivation der } \\
\text { Ratsuchenden. } \\
\text { Erste Strukturie- } \\
\text { rung der Pro- } \\
\text { blemlagen. } \\
\text { Treffsicherheit } \\
\text { der Aufnahme in } \\
\text { das Case Ma- } \\
\text { nagement. }\end{array}$ \\
\hline \multicolumn{4}{|c|}{ Denkbare Teilschritte } \\
\hline \multicolumn{4}{|c|}{$\begin{array}{l}\text { Gewinnen Sie ein erstes Bild der Grundproblematik und der Ressourcen. } \\
\text { Gewinnen Sie einen Eindruck der Motivation der bzw. des Ratsuchen- } \\
\text { den. } \\
\text { Entscheiden Sie über die Aufnahme in das Case Management. } \\
\text { Klären Sie über den Prozess des Case Managements und die damit ver- } \\
\text { bundenen Chancen, Anforderungen und Ihre Rolle auf. } \\
\text { Schließen Sie einen ersten mündlichen Kontrakt. }\end{array}$} \\
\hline
\end{tabular}




\subsubsection{Assessment}

Im Prozess des Case Managements soll zweierlei geklärt werden: Zum einen, ob die Bedarfslage so komplex ist, dass das Handlungskonzept Case Management überhaupt indiziert ist. Zum anderen soll ein differenziertes Bild der Ausgangslage einer Adressatin bzw. eines Adressaten gewonnen werden. Für die erstgenannte Funktion wird in der Literatur meist der Begriff des „Screening" oder auch „Profiling“, für die zweite Funktion der des „Assessments“ verwendet. „Screening“ benennt somit das „Zugangsmanagement" zum speziellen Angebot Case Management, trifft aber noch keine differenzierte Aussage zu Problem- und Bedarfslagen. Demgegenüber geht es im Assessment darum, jede Adressatin bzw. jeden Adressaten als „sozialen Fall“ zu rekonstruieren, um aus dieser Rekonstruktion Probleme erkennen und über deren Lösung nachdenken zu können. Im Rahmen einer ausführlichen Befragung der Adressatin oder des Adressaten wird über deren bzw. dessen Versorgungsdefizite und Versorgungswünsche ebenso gesprochen wie über eventuell vorhandene Bewältigungsressourcen.

Das Assessment ist für den gesamten Prozess des Case Managements von ausschlaggebender Bedeutung, da die hier gesammelten Daten und Informationen die Grundlage für einen bedarfsorientierten individuellen Hilfeplan bilden. In der anglo-amerikanischen Literatur wird zwischen „problemorientiertem“ und „bedarfsorientiertem“ Assessment unterschieden („risk-“ vs. „needs-assessment“), wobei der Unterschied vor allem in der Betonung der Zielorientierung der Datensammlung liegt. Insgesamt ist davor zu warnen, das Themenfeld zu eng abzustecken. Wie aus der Arbeitslosenforschung bekannt ist, kann Arbeitslosigkeit auf mehrere Lebensbereiche und vor allem die Selbstwahrnehmung der Betroffenen zurückwirken. So können Alltagsprobleme, die „unter normalen Umständen“ leicht zu bewältigen wären, eine solche Bedeutung erlangen, dass sie die Beschäftigungsfähigkeit und damit Vermittlungsfähigkeit herabsetzen. Dies macht dann das Erkennen und die Bearbeitung anderer Probleme erforderlich, die vordergründig nichts mit Erwerbstätigkeit zu tun haben. Die Datensammlung sollte „zielorientiert“ erfolgen, damit sie nicht zum Selbstzweck wird und auf diese Weise lediglich Kontroll- bzw. Erziehungseffekte erzielt/erzielen soll. So steht z. B. im Fokus des „Fallmanagements“ im SGB II das Ziel der Aufnahme einer Beschäftigung, das Assessment wird sich also schwerpunktmäßig auf Fragen der Erwerbsfähigkeit richten. Bei einem Assessment in anderen (Rechts-)Bereichen wird der Fokus dagegen breiter sein. Hieraus ist die Anforderung abzuleiten, diese Phase so zu gestalten, dass ein themenzentriertes Gespräch geführt wird, das aber offen 
bleibt für Deutungen der Lebenssituation durch die Betroffenen. Um diese Offenheit zu erreichen, ist es sinnvoll, „Checklisten“ zu verwenden, die den „roten Faden“ des Gesprächs gewährleisten, gleichzeitig aber nicht zu schematisch angelegt sein sollten. Mit diesen Checklisten wird nämlich eine Praxis befördert, die systematisch als „Profiling“ bekannt ist und deutliche Unterschiede zum Assessment aufweist, wie es hier skizziert wird. Checklisten und Diagnosebögen dienen dazu, Adressatinnen und Adressaten erstmals und vorläufig bestimmten Kategorien zuzuordnen. Ausschlaggebend hierfür ist die Verknüpfung der Daten und Informationen mit bestimmten Merkmalen der Kategorien, um z. B. Auskunft zu erhalten über die Schwierigkeiten der Vermittlung bzw. die geeigneten Maßnahmen, um die Vermittlungsfähigkeit zu verbessern. Zur Gewinnung eines exakten Bilds reicht es jedoch nicht aus, Checklisten mit vorgefertigten Antwortrastern auszufüllen, auch wenn diese hilfreich sein können, um wichtige Daten zu erfassen. Das Bild einer Person wird erst vollständig, wenn auch deren subjektive Perspektiven, ihre Wünsche und Träume berücksichtigt werden - eine Dimension, die sich im Gespräch nicht erschließt, wenn schematisch Daten abgefragt werden. Ein Assessment kann sich nicht darin erschöpfen, Daten abzufragen und zu dokumentieren, es verlangt vielmehr einen spezifischen Interaktionsprozess zwischen den Professionellen und ihren Adressatinnen und Adressaten. ${ }^{52}$ Die Qualität des Assessments ist das zentrale Thema einer Studie, die Thompson und Mikelson veröffentlicht haben. Sie zeigen, dass die Aufgabe des Assessments nur dann gelingen kann, wenn eine vertrauensvolle Atmosphäre geschaffen ist: „Case Manager müssen über eine Zeit hinweg eine Vertrauensbasis zu ihren Klienten entwickeln und sie sollten in den einzelnen Treffen immer wieder Themen der Sicherheit und Vertrauenswürdigkeit ansprechen" (Thompson/Mikelson 2001: S.35). Da der Aufbau von Vertrauen auch eine Frage der aufgewandten Zeit ist, sind zu früh ansetzende AssessmentVerfahren nur bedingt geeignet bzw. wirken selektiv (zu Ungunsten von Personen mit besonderen Schwierigkeiten). Außerdem sollte ein Assessment immer nur gemeinsam mit der Adressatin bzw. dem Adressaten erfolgen. In ähnliche Richtung weisen die Leitlinien des britischen Gesundheitsministeriums zum Assessment in sozialen Diensten: Auch dort wird der Transparenz und Partizipation ein hoher Stellenwert zugewiesen (vgl. Milner/O'Byrne 2002).

Der Prozessschritt Assessment umschließt somit die zielgerichtete Erfassung bestimmter Daten wie auch das offene Gespräch, in dem die Subjek-

52 Einen aktuellen Überblick über Assessmentinstrumente gibt Brühl 2019. 
tivität der Ratsuchenden zur Geltung kommen kann. Hinzu kommt (und das macht den Kern des Assessments aus), dass all diese Informationen interpretativ zusammengefasst werden müssen - und zwar gemeinsam mit den adressierten Personen. Auf diese Weise wird deren „status quo“ wie auch ihre Perspektive rekonstruierbar und bildet das „Ausgangsmaterial“, um hieraus Ziele formulieren zu können.

Methodisch gibt es viele Möglichkeiten, ein Assessment durchzuführen. Als Grundregeln sollten gelten:

- Größtmögliche Transparenz in der Datenerhebung,

- keine Eingabe in die EDV während einer laufenden Beratung,

- Interpretationen nur gemeinsam mit den Adressatinnen und Adressaten

(vgl. auch Deutscher Verein für öffentliche und private Fürsorge 2004).

Das Assessment kann schematisch folgendermaßen dargestellt werden.

2. Stufe: Assessment

\begin{tabular}{|c|c|c|c|}
\hline iel & & & \\
\hline $\begin{array}{l}\text { r- } \\
d\end{array}$ & $\begin{array}{l}\text { en } \\
\text { en }\end{array}$ & $\begin{array}{l}\text { r } \\
\text { Di- }\end{array}$ & \\
\hline \multicolumn{4}{|l|}{ Denkb } \\
\hline \multicolumn{4}{|c|}{$\begin{array}{l}\text { Legen Sie den Schwerpunkt bei der Datenerhebung auf die konkreten } \\
\text { Bedarfslagen der Adressatin bzw. des Adressaten. } \\
\text { Beziehen Sie die Adressatin bzw. den Adressaten, evtl. auch ihre bzw. sei- } \\
\text { ne Angehörigen und übrige Fallbeteiligte, in die Datenerhebung ein. } \\
\text { Schreiben Sie die Datenerhebung systematisch und kontinuierlich fort. } \\
\text { Dokumentieren Sie relevantes Datenmaterial in verständlicher Form. } \\
\text { Leiten Sie Interpretationen (z. B. Kategorisierungen) aus den Assessment- } \\
\text { Daten ab. } \\
\text { Bestätigen Sie die Interpretationen gemeinsam mit der Adressatin bzw. } \\
\text { dem Adressaten, ihren bzw. seinen Angehörigen und übrigen Fallbetei- } \\
\text { ligten und entwickeln so ein (gemeinsames) Fallverständnis. } \\
\text { Halten Sie Abweichungen in der Interpretation fest. } \\
\text { Dokumentieren Sie das Assessment, das die Definition von Zielen und } \\
\text { die Erstellung von Hilfeplänen erleichtert. }\end{array}$} \\
\hline
\end{tabular}




\subsubsection{Zielvereinbarung}

Die Hilfeplanung wird eingeleitet durch den Prozessschritt der Vereinbarung von Zielen zwischen Fachkräften und Ratsuchenden. Dieser Schritt stellt einerseits einen integralen Bestandteil von Hilfeplanung bzw. dem weitergehenden Case Management dar, kann aber auch analytisch und logisch getrennt betrachtet werden.

An dieser Stelle kann die komplexe Problematik der Formulierung und Vereinbarung von Zielen nur kursorisch werden. In jedem einzelnen Fall, in dem ein Ziel zwischen Case Manager und Adressatin oder Adressaten vereinbart wird, geht es darum, für ein mehr oder minder komplexes, aber eben einzelfallbezogenes Problem Lösungswege zu suchen. Hierzu wird idealerweise gemeinsam und formalisiert - zwischen Case Manager und Adressatin oder Adressaten ein Ziel vereinbart: Die Existenz soll gesichert, eine Wohnung gefunden, eine Arbeit angetreten, eine Ausbildung abgeschlossen werden. Die Handlungsebene des Einzelfalls ist jedoch auf der organisationalen Seite eingebunden in eine explizit formulierte Zielhierarchie, die Fachkräfte im Case Management mehr oder weniger deutlich reflektieren. Die differenzierte Betrachtung unterschiedlicher Zielebenen und -perspektiven kann für die Klärung des komplexen Prozesses der Zielbildung im Rahmen von Hilfeplanung bzw. in Beratungsgesprächen genutzt werden. Denn auch für die Beratung und Hilfeplanung im Rahmen der Beschäftigungsförderung gilt, was Gerth, Menne und Roth für die Erziehungsberatung feststellen: Sie wird „i. d. R. wegen konkreter Problemlagen in Anspruch genommen, die dafür auslösend waren, die Hilfe einer Beratungsstelle zu suchen. Der Erfolg einer Beratung kann daher gesehen werden, wenn sich bezogen auf die Ausgangssituation Veränderungen ergeben haben (...). Allerdings verändern sich die zunächst genannten Anlässe auch im Laufe einer Beratung (...). Der objektive Erfolg von Beratung lässt sich deshalb nicht direkt als Problemveränderung erfassen. Er kann immer nur bezogen werden auf die Problemdefinition bzw. -redefinition, die zu Beginn einer Beratung ausgehandelt wurde" (Gerth/Menne/Roth 1999: S. 65). Nimmt man diese Perspektive ein, sind Beratungserfolge immer nur in Relation zu den zwischen Beratenden und Ratsuchenden ausgehandelten Zielen zu definieren, „Effektivität“ (Grad der Zielerreichung) und „Effizienz“ (Mitteleinsatz im Verhältnis zum erreichten Ziel) nur im Rekurs auf die Interaktion zwischen Beratenden und Ratsuchenden und ihre Geschichte (da sich die Rahmenziele im Prozess verändern können) bestimmbar. Dies hat zur Konsequenz, dass eine Auswertung (Evaluation) der Zielerreichung zwingend fallrekonstruktiv sein muss (was über eine 
entsprechende Dokumentation gelingen kann) und außerdem die Perspektive der oder des Ratsuchenden einbeziehen muss (z. B. durch Nachbefragungen).

Die Tatsache, dass Ziele ko-produktiv im Rahmen der Interaktion zwischen Beratenden, Adressatinnen und Adressaten ausgehandelt werden, kann jedoch nicht überdecken, dass sie aus der Organisationsperspektive Bestandteil eines komplexen Zielsystems ist. Gerade komplexere Hilfeleistungen greifen weit über „Beratung“ hinaus und können auch stärker in die institutionelle und organisationelle Zielperspektive eingebunden sein. Dies bedeutet, dass für die Analyse konkreter Abläufe sowie die Auswertung (Evaluation), die auf dieser Ebene ansetzt, die Dimensionen der rechtlich vorgegebenen oder organisatorisch gesetzten „Leitziele“ und „strategischen Ziele“ nicht außer Acht gelassen werden dürfen.

Zielvereinbarungen sind ein schwieriges Geschäft für die Professionellen, weil sie sowohl die interaktive Ebene im Blick haben und hier auf einen Konsens hinarbeiten müssen, als auch eine Vermittlungsleistung zu den Interessen des Auftragsgebenden zu erbringen haben. Sie stecken somit in einem „strategischen Dilemma“, was sich als Beratungsdilemma in den Interaktionen wiederfinden kann (vgl. 5.3.1). Auf alle Fälle zu berücksichtigen ist die biografische Erfahrung der adressierten Personen. Interventionen können unterschiedlich stark in das bisherige Leben eingreifen.

- Hilfe ist konform zur aktuellen Lebensweise (biografiekonform, daher im Wesentlichen matching-Problem).

- Hilfe ist diskrepant zur aktuellen Lebensweise (Erwerbsarbeit ist nur bedingt vereinbar mit anderen Lebensbereichen wie der häuslichen Situation, den Lebensvorstellungen - z. B. Ausbildung vs. Erwerbsarbeit, Wohnortwechsel; Hilfeziele bedeuten für den Jugendlichen ein „Aufbrechen zu neuen Ufern").

- Hilfe ist konträr zur aktuellen Lebensweise (aktuelle Lebenssituation lässt Erwerbsarbeit oder Ausbildung nicht zu. Der Jugendliche hat sich in einer bestimmten Lebensweise eingerichtet bzw. ist dort hineingewachsen, die als individuelle Überlebensstrategie zu verstehen ist. Dies können sein: U. a. Familientraditionen eines randständigen Lebens; psychosoziale Auffälligkeiten - hier besteht wenig Spielraum für zusätzliche Belastungen; Sucht; Obdachlosigkeit).

Diese Kategorien stammen aus der Forschung zum Case Management in der Beschäftigungsförderung (vgl. Reis/Hobusch/Kolbe 2011), sind aber sehr gut auf andere Zielgruppen zu übertragen. Teilhabekompetenzen und Bewältigungsressourcen von Adressatinnen und Adressaten sind bei der Zielvereinbarung so zu berücksichtigen, dass Ziele motivieren und nicht 
absehbar zu Misserfolgen führen. Deshalb hängt die Qualität der Zielvereinbarung unmittelbar mit der Qualität des Assessments und der damit verbundenen Einschätzung der Ressourcen und Potenziale der adressierten Personen zusammen.

Parallel zu diesen interaktiven Momenten einer Zielvereinbarung ist deren rechtliche Einbettung zu beachten. Ein wesentliches Element des Case Managements ist die Kooperation verschiedener Behörden und sonstiger Akteure, unter Umständen die Durchführung von Fallkonferenzen. Dies beinhaltet einen erheblichen Datenfluss, womit unmittelbar elementare datenschutzrechtliche Regelungen ins Spiel kommen. Sinnvoll ist vor diesem Hintergrund im Falle des rechtskreisübergreifenden Case Managements der Abschluss einer schriftlichen Integrationsvereinbarung, die inhaltlich die Anforderungen einer Zielvereinbarung erfüllt und gleichzeitig alle datenschutzrechtlichen Vorschriften bedient.

3. Stufe: Zielvereinbarung

\begin{tabular}{|c|c|c|c|}
\hline Ziel & \begin{tabular}{|l} 
Benötigte \\
Ressourcen
\end{tabular} & Grundproblem & $\begin{array}{l}\text { Erfolgs- } \\
\text { kriterium }\end{array}$ \\
\hline $\begin{array}{l}\text { Für beide Seiten } \\
\text { verbindliche } \\
\text { Festlegung, was } \\
\text { erreicht werden } \\
\text { soll }\end{array}$ & $\begin{array}{l}\text { Aushandlungs- } \\
\text { kompetenz } \\
\text { Dokumentati- } \\
\text { ons-system, das } \\
\text { geeignet ist, un- } \\
\text { terschiedliche } \\
\text { Zielebenen fest- } \\
\text { zuhalten }\end{array}$ & $\begin{array}{l}\text { Differierende } \\
\text { Ziele („strategi- } \\
\text { sches Dilemma“) }\end{array}$ & $\begin{array}{l}\text { Tragfähige, d. h. } \\
\text { realistische Ziel- } \\
\text { vereinbarung } \\
\text { Erstes Konzept } \\
\text { der Zielerrei- } \\
\text { chung }\end{array}$ \\
\hline \multicolumn{4}{|c|}{ Denkbare Teilschritte } \\
\hline \multicolumn{4}{|c|}{$\begin{array}{l}\text { Leiten Sie die Ziele aus dem Assessment ab. } \\
\text { Dokumentieren Sie die erwarteten Resultate als messbare Zielsetzungen. } \\
\text { Entwickeln Sie Zielsetzungen gemeinsam mit der Adressatin bzw. dem } \\
\text { Adressaten und evtl. den übrigen Fallbeteiligten. } \\
\text { Entwickeln Sie realistische Ziele in Relation zu den aktuellen und künfti- } \\
\text { gen Möglichkeiten der Adressatin bzw. des Adressaten. } \\
\text { Legen Sie einen Zeitrahmen fest, in dem die Ziele erreicht werden sol- } \\
\text { len. } \\
\text { Entwickeln Sie Ziele, die Orientierung vermitteln in Richtung auf die } \\
\text { Kontinuität des Hilfeprozesses. }\end{array}$} \\
\hline
\end{tabular}




\subsubsection{Hilfeplanung}

Die Hilfeplanung stellt einen notwendigen Schritt im Rahmen des Case Managements dar, sie folgt - als eigenständige Handlungsform - einer eigenen Logik, die auch außerhalb des Case Managements beachtet werden sollte.

Der Unterschied zwischen „Erstberatung und Assessment“ sowie „Hilfeplanung" besteht darin, dass im Rahmen der Hilfeplanung Zukunft „geplant" wird und nicht so offen ist wie im Rahmen von Beratungsprozessen. Hieraus resultiert eine spezifische „Logik“ von Hilfeplanung:

- Wenn Zukunft „geplant“ wird, bedeutet dies (im Gegensatz zu einem unverbindlichen Gespräch), dass alle Beteiligten davon ausgehen, dass der Plan auch realisiert werden kann, der Plan ist somit ein Entwurf des Wegs zu einem künftigen Zustand.

- Dieser Zustand muss möglichst exakt beschrieben werden, damit der Weg dorthin nachvollziehbar abgeleitet werden kann. Diese Beschreibung erfolgt über die Formulierung von Zielen, die operationalisierbar sein müssen. Insofern ist die implizite oder explizite Vereinbarung von Zielen Bestandteil von Hilfeplanung und von dieser nur analytisch trennbar.

- Die Planung verlangt neben der Zielformulierung auch das Abstecken eines Zeitrahmens und die Festlegung von Rollen, Aufgaben und Verantwortungsbereichen.

- Hilfeplanung ist somit die gedankliche Vorwegnahme künftiger und gewünschter Zustände (d. h. Zielformulierung) und des Wegs zu deren Erreichung. Darüber hinaus erscheint es systematisch sinnvoll, auch das Monitoring, d. h. die fortlaufende Überprüfung der Zielerreichung, zur Hilfeplanung zu zählen, denn ein Hilfeplan ist kein statisches Instrument, sondern nur dann brauchbar, wenn er modifiziert bzw. fortgeschrieben wird, indem die tatsächlichen Entwicklungen (d. h. die reale Zukunft im Gegensatz zur vorgestellten) reflektiert und zum Anlass weiterer Planungen genommen werden.

- Dagegen gehört die Steuerung der Umsetzung, die konkrete Vermittlung von Hilfsangeboten und deren Koordination nicht zur Hilfeplanung, da sie einer anderen Handlungslogik folgt (vgl. den Prozessschritt „Leistungssteuerung“). 
Diese Handlungslogik hat Folgen für die Gestaltung von Hilfeplanung, die folgende zwei „Essentials“ aufweisen sollte:

- Die möglichst konkrete Formulierung von Teilschritten, die deren Überprüfbarkeit mitreflektiert. Nur auf diese Weise können Abweichungen festgestellt und reflektiert werden.

- Die Einbeziehung der Adressatinnen und Adressaten in die Hilfeplangestaltung, denn nur der - teilweise allein über „Aushandlungen“ erreichbare - Konsens über den Hilfeplan und seine Umsetzung macht deren Erreichung wahrscheinlich. Wenn aber die Realisierung nicht von Beginn an erwartet werden kann, ist die Hilfeplanung sinnlos. Hieraus resultiert die Anforderung, realistische, d. h. der aktuellen Situation und den Möglichkeiten der Adressatinnen und Adressaten angemessene, Teilschritte zu formulieren.

Gerade das letztgenannte „Essential“ birgt das oben skizzierte Dilemma, dass die Übereinstimmung der Ziele aller am Prozess Beteiligten nicht vorausgesetzt werden kann. Dies bedeutet, dass ein Konsens per „Aushandlung" erreicht werden muss, der für diese Beteiligten tragfähig ist. Geschieht dies nicht, sollte nicht von Hilfeplanung gesprochen werden.

Um mit Konsens und Dissens in der Hilfeplanung systematisch umgehen zu können, sollten nicht nur die vereinbarten Ziele und Realisierungsschritte, sondern auch abweichende Positionen dokumentiert werden. Damit wird

- signalisiert, dass auch abweichende Standpunkte ernst genommen werden,

- sichergestellt, dass Widerstände, die im Dissens verborgen liegen, erkennbar und vielleicht bearbeitbar werden und

- eine Voraussetzung für die weitere Auseinandersetzung mit dem Dissens geschaffen.

Was für die Zielformulierung und -vereinbarung gilt, gilt auch für die Planung der einzelnen Handlungsschritte. Ein wichtiges Element im Aushandlungsprozess ist die Festlegung von Aufgaben und Verantwortungsbereichen sowie unterschiedlicher Rollen. Auch hier sollte das Prinzip beachtet werden, dass die einzelnen Aufgaben möglichst konkret benannt, Verantwortlichkeiten festgelegt und Kriterien der Aufgabenerfüllung im Konsens erarbeitet werden. Ebenso wichtig ist es bei der Hilfeplanung, zweitbeste Wege, die gegangen werden müssen, weil geeignete Angebote und Maßnahmen für den besten Weg fehlen, als solche zu markieren. Die fehlenden Angebote und Maßnahmen müssen dann beschrieben und in die Organisation hinein als zu deckender künftiger Bedarf gemeldet werden. 
Hier verknüpfen sich einzelfallbezogene Hilfeplanung und einzelfallübergreifende Angebotsplanung.

4. Stufe: Hilfeplanung

\begin{tabular}{|c|c|c|c|}
\hline Ziel & \begin{tabular}{|l|} 
Benötigte \\
Ressourcen
\end{tabular} & Grundproblem & \begin{tabular}{|l|} 
Erfolgs- \\
kriterium
\end{tabular} \\
\hline $\begin{array}{l}\text { Gemeinsame } \\
\text { Planung des Ein- } \\
\text { satzes von Hilfe- } \\
\text { angeboten und } \\
\text { Ressourcen. }\end{array}$ & \begin{tabular}{|l|} 
Verhandlungs- \\
kompetenz. \\
Fähigkeit zu rea- \\
listischer Ein- \\
schätzung von \\
Bewältigungs- \\
ressourcen. \\
Überblick über \\
formelle und in- \\
formelle Hilfsan- \\
gebote.
\end{tabular} & $\begin{array}{l}\text { Unrealistische } \\
\text { Einschätzung } \\
\text { von Angeboten } \\
\text { und Bewälti- } \\
\text { gungsressourcen } \\
\text { (von beiden Sei- } \\
\text { ten). }\end{array}$ & $\begin{array}{l}\text { Realistischer Hil- } \\
\text { feplan, der zwi- } \\
\text { schen Bedarf, Be- } \\
\text { wältigungs-res- } \\
\text { sourcen und An- } \\
\text { gebot vermittelt. }\end{array}$ \\
\hline \multicolumn{4}{|c|}{ Denkbare Teilschritte } \\
\hline \multicolumn{4}{|c|}{$\begin{array}{l}\text { Arbeiten Sie einen auf die Bedarfslagen der Adressatin bzw. des Adressa- } \\
\text { ten individuell zugeschnittenen Hilfeplan aus. } \\
\text { Entwickeln Sie den Hilfeplan gemeinsam mit der Adressatin bzw. dem } \\
\text { Adressaten, evtl. ihren bzw. seinen Angehörigen und den übrigen Fallbe- } \\
\text { teiligten. } \\
\text { Legen Sie den Hilfeplan schriftlich nieder. } \\
\text { Stellen Sie in ihrem Hilfeplan die Kontinuität der Hilfe sicher, indem Sie } \\
\text { optionale und alternative Vorgehensweisen benennen. }\end{array}$} \\
\hline
\end{tabular}

\subsubsection{Leistungssteuerung}

Case Management unterscheidet sich von der klassischen sozialarbeiterischen Einzelfallhilfe („,case work“) dadurch, dass die Bearbeitung und ggf. Lösung erkannter Probleme nicht von den Fachkräften im Case Management selbst in Angriff genommen werden, sondern diese hierzu Leistungen „Dritter“ in Anspruch nehmen, d. h. den Fokus ihrer Arbeit in der kompetenten Vermittlung und Steuerung des richtigen Angebots sehen. Die Qualität von Case Management bemisst sich damit an der Kompetenz, Hilfsangebote so zu vermitteln, dass sie auf die besondere Bedarfslage des Einzelfalls zugeschnitten sind. Dies setzt voraus, dass Case Manager Steue- 
rungskompetenzen gegenüber den Leistungsträgern haben, deren Angebote in Anspruch genommen werden sollen. Leistung ist dann besonders flexibel zu steuern, wenn potenzielle Anbieter über Verträge eingebunden sind und die Professionellen über Budgets verfügen, die sie fallspezifisch einsetzen können. Allerdings sollte beachtet werden, dass diese Handlungsautonomie in ein Steuerungskonzept eingebettet sein muss, soll sie nicht in Willkür umschlagen.

Generell muss die Steuerung der Leistungen Dritter Bestandteil eines umfassenden Controlling-Konzepts sein. Genau hier stellen sich Probleme in der Kooperation mit „Dritten“. Auch wenn Adressatinnen und Adressaten an Dritte (seien es Beratungsstellen oder Beschäftigungsträger) weiterverwiesen wurden, haben die Fachkräfte im Case Management die Fallverantwortung und dies bedeutet, dass sie nicht nur wissen müssen, „wo“ die Adressatinnen und Adressaten sich befinden, sondern auch stets (unter Beachtung der datenschutzrechtlichen Vorschriften) über den Stand der Beratung oder Maßnahme informiert sein müssen. Im Rahmen der Leistungssteuerung müssen deshalb Monitoring- und Rückmeldesysteme entwickelt werden, die innerhalb des Netzwerks abgestimmt sind, d. h. sowohl die Zielorientierung des Case Managements als auch die fachlichen Kriterien der Kooperationspartnerinnen und -partner berücksichtigen.

Je nach Art der Leistungsangebote stellen sich bei der Leistungssteuerung besondere Pro-bleme. In der Regel hat Case Management seinen Ausgangspunkt in einem Rechtskreis (z. B. des SGB II). Da es sich aber an Personen mit multiplen Vermittlungshemmnissen wendet, liegt es in der $\mathrm{Na}-$ tur der Sache, dass Personen Unterstützung aus anderen Rechtskreisen und weitere Angebote benötigen. Für die meisten dieser Angebote existieren fachliche Standards, zudem werden sie meist von Institutionen mit ausgeprägtem Eigeninteresse, eigenständigen Wertorientierungen oder eigenem gesetzlichen Auftrag (z. B. die Jugendhilfe mit dem SGB VIII) organisiert. Hier müssen die Case Manager ihre fachliche und institutionelle Eigenlogik beachten, soll die Kooperation mit den entsprechenden Trägern nicht von vornherein scheitern. So ist es z. B. notwendig, dass in der Schuldnerberatung eigenständige professionelle Beziehungen zu überschuldeten Adressatinnen und Adressaten aufgebaut werden und nach eigenen fachlichen Standards auf das Ziel Entschuldung hingearbeitet wird - in Zeitdimensionen, die nicht extern gesetzt werden können, sondern fallabhängig sind. Deshalb können (und müssen) zwischen Case Management und Schuldnerberatung Ziele vereinbart werden. Diese dürfen die eigenständige fachliche Arbeit in der Schuldnerberatung aber nicht gefähr- 
den (z. B. durch enge zeitliche Vorgaben), sondern müssen sich mit deren fachlicher Perspektive in Einklang bringen lassen.

Steuerung kann sich hier nicht alleine des Mediums Geld bedienen, vielmehr erfordert sie eine kontinuierliche inhaltliche und fallbezogene (aber auch: zielorientierte) Kommunikation zwischen Fachkräften mit unterschiedlichem professionellen Hintergrund. Dies gilt erst recht dann, wenn die Leistungsanbieter ihre Arbeit an gesetzlichen Zielen ausrichten, die nicht dieselben wie die des Case Managements sind. So orientiert sich z. B. der ASD in der Gewährung von „Hilfe zur Erziehung“ am „Wohl“ der Kinder bzw. Jugendlichen und dies kann, muss aber nicht mit der Zielsetzung des SGB II kompatibel sein, Jugendliche rasch in Ausbildung oder Beschäftigung zu integrieren. Es bedarf hier offensichtlich intensiver fallbezogener Kooperation. Als ein geeignetes Instrument zur kooperativen Bewältigung von Pro-blemlagen hat sich die (unter Umständen multidisziplinäre) Fallkonferenz erwiesen.

Insgesamt muss Case Management als Kombination von Einzelfall- und Netzwerkarbeit begriffen werden. Dabei bleibt zu beachten, dass die einzelnen Case Manager mit einem Netzwerk arbeiten und dieses auch pflegen müssen, der Aufbau und die Stabilisierung des Netzwerks jedoch eine Aufgabe im Rahmen der Systemsteuerung darstellt. Die für einzelne Hilfsangebote in Frage kommenden Träger müssen frühzeitig eingebunden werden, und zwar in der doppelten Form von rechtlicher Einbindung (sei es über Zuwendungsrecht oder im Rahmen öffentlicher Aufträge, d. h. des Vergaberechts) und sozialpolitischer Kooperation. Leistungssteuerung im Netzwerk kann sich explizit nicht auf die Steuerung von "Zuliefernden“ beschränken wie z. B. in der Industrie, sondern bedeutet den Rückgriff auf und die Weiterentwicklung von kommunaler sozialer Infrastruktur. Welche Netzwerkpartner wie einzubinden sind, ist von der Bedarfslage der Zielgruppe und der vorhandenen sozialen Infrastruktur in den jeweiligen Kommunen abhängig, denn in Deutschland existieren unterschiedliche Kooperationskulturen zwischen unterschiedlichen Bereichen der kommunalen Sozialverwaltung (Sozialamt, Jugendamt, Gesundheitsamt, Ausländerbehörde etc.), den örtlichen Gliederungen der Verbände der freien Wohlfahrtspflege, der Arbeitsverwaltung, Selbsthilfeorganisationen, Schulen und Wirtschaft. Es empfiehlt sich, frühzeitig mit allen relevanten Akteuren in ein Gespräch zu kommen und - z. B. für bestimmte Fallkonstellationen - idealtypische Leistungspakete zu entwerfen. Werden diese geschnürt, sollte der zeitliche Ablauf geplant werden. Manche Angebote sind sinnvollerweise hintereinander zu schalten, manche können parallel laufen. Bestandteil des Hilfeplans sollte deshalb auch eine Verlaufsplanung 
sein, die sich der Netzplantechnik bedienen könnte. Die Problemlagen sind unter Umständen auch so spezifisch oder komplex, dass deren Bearbeitung nicht nur spezielle fachliche Kompetenzen, sondern zusätzliche Koordinationsleistungen erfordert (etwa im Falle von Suchtkranken oder Wohnungslosen). Dann wäre ein spezielles zielgruppenbezogenes Case Management zu konzipieren, das eingeschaltet werden kann, um die geeigneten Angebote zu entwerfen und im Auftrag des ursprünglichen Case Managements zu steuern. Elementar für das Case Management sind fundierte Kenntnisse über die vorhandene Angebotspalette (z. B. in Form von Anbieterdatenbanken) und regelmäßige Treffen mit den einzubeziehenden Akteuren sowohl auf Leitungs- als auch auf Mitarbeitendenebene.

Wird Case Management in einer Organisation neu installiert, ist es wichtig, die Kontakte zu „Dritten“ zu fördern und schließlich zu systematisieren. Sonst besteht die Gefahr, dass weniger als möglich und notwendig auf die Angebote Dritter zurückgegriffen wird. Dazu gehören auf der übergeordneten Ebene die Steuerung Dritter durch Leistungsverträge genauso wie bspw. das Ermöglichen von Hospitationen zwischen Fachkräften im Case Management und den Kolleginnen und Kollegen freier Träger untereinander auf der Arbeitsebene. In der Praxis kann häufig beobachtet werden, dass sich die Kontakte mit der jeweils relevanten Trägerlandschaft nach Einführung des Case Managements kontinuierlich intensivieren. Auf diese Weise werden die bestehenden Kooperationsbeziehungen systematisiert. Ein Beispiel ist die Praxis, freie Träger in Fallkonferenzen miteinzubeziehen, analog zu Hilfeplankonferenzen nach $\$ 36$ SGB VIII. Unterhalb dieser Ebene kann es eine Reihe formeller und informeller Absprachen zwischen den Fachkräften im Case Management und Leistungserbringenden geben. Das Case Management wird es in vielen Fällen nicht nur mit einzelnen Leistungsträgern zu tun bekommen, die im Rahmen bilateraler Kooperationsbeziehungen einzelne Aufgaben übernehmen, sondern gerade in komplexen Fallkonstellationen mit Netzwerkstrukturen konfrontiert sein. So bilden sich auf lokaler Ebene unter Umständen „Produktionsnetzwerke", die gemeinsam Dienstleistungen erbringen. Diese kompetent zu steuern, ist eine notwendige, aber auch voraussetzungsvolle Aufgabe, die kompetent wahrgenommen werden muss, um effektives Case Management zu realisieren. Sie erfordert aber genau deshalb so viel Zeit, dass Netzwerkmanagement nicht personell kombinierbar ist mit Case Management, sondern parallel hierzu realisiert werden sollte. 
5.Stufe: Leistungssteuerung

\begin{tabular}{|c|c|c|c|}
\hline Ziel & \begin{tabular}{|l} 
Benötigte \\
Ressourcen
\end{tabular} & Grundproblem & $\begin{array}{l}\text { Erfolgs- } \\
\text { kriterium }\end{array}$ \\
\hline $\begin{array}{l}\text { Das Leistungsan- } \\
\text { gebot wird be- } \\
\text { darfsgerecht er- } \\
\text { bracht. }\end{array}$ & \begin{tabular}{|l|} 
Kooperations- \\
kompetenz. \\
Moderations- \\
kompetenz. \\
Kompetenz, An- \\
gebote miteinan- \\
der zu verknüp- \\
fen. \\
Bedarfsgerech- \\
tes, zeitnah be- \\
reitstehendes \\
Angebot. \\
Einbindung der \\
Leistungsanbie- \\
ter in das Case \\
Management- \\
Konzept.
\end{tabular} & $\begin{array}{l}\text { Fehlende bzw. } \\
\text { nicht ausrei- } \\
\text { chend zeitnah } \\
\text { verfügbare Ange- } \\
\text { bote. } \\
\text { Hoher Koordina- } \\
\text { tions-aufwand } \\
\text { aufgrund struk- } \\
\text { tureller Unab- } \\
\text { hängigkeit der } \\
\text { Leistungsanbie- } \\
\text { ter. }\end{array}$ & $\begin{array}{l}\text { Zeitnahe Be- } \\
\text { darfs-deckung. } \\
\text { Kontinuierliche } \\
\text { Koordination } \\
\text { zwischen indivi- } \\
\text { duellem Case } \\
\text { Management } \\
\text { und Angebots- } \\
\text { planung. }\end{array}$ \\
\hline \multicolumn{4}{|c|}{ Denkbare Teilschritte } \\
\hline \multicolumn{4}{|c|}{$\begin{array}{l}\text { Suchen Sie den Kontakt zu den beteiligten Fachkräften bei den Leis- } \\
\text { tungserbringende. } \\
\text { Etablieren Sie einen kontinuierlichen fallspezifischen (z. B. Fallkonferen- } \\
\text { zen) und fallübergreifenden bzw. -unspezifischen Austausch der (poten- } \\
\text { ziell) Fallbeteiligten. } \\
\text { Klären Sie die faktischen, finanziellen und juristischen Modalitäten der } \\
\text { Vermittlung. } \\
\text { Wenn nötig, wirken Sie darauf hin, fallübergreifende Regelungen mit } \\
\text { den Leistungserbringenden zu entwickeln. }\end{array}$} \\
\hline
\end{tabular}

\subsubsection{Monitoring der Leistungserbringung}

Ein zentrales Charakteristikum von Case Management ist die auf eine Hilfeplanung gestützte Vermittlung der (Dienst-)Leistungen Dritter (vgl. Leistungssteuerung, Abschnitt 5.4.5). Auch wenn dies gelungen ist, bleiben die Case Manager fallverantwortlich, d. h. müssen stets den Überblick über 
den Gesamtprozess haben und den „Fall“ begleiten. Daneben muss es möglich sein, dass Ratsuchende ein besonderes Vertrauensverhältnis zu „ihren“ Case Managern entwickeln und weiterhin - diesen Beratungskontakt halten wollen. In solchen Fällen werden Case Manager entsprechende Beratungsleistungen erbringen müssen, die sie nicht an „Dritte“ delegieren können.

Systematisch reflektiert wird die Begleitung im Monitoring. Dieses ist Teil des Unterstützungsprozesses im Einzelfall und meint das Nachhalten und Überprüfen des vereinbarten Vorgehens - gemeinsam mit den Ratsuchenden. Ziel des Monitorings ist es, mögliche Fehleinschätzungen oder Fehlentwicklungen frühzeitig zu entdecken, um diese dann zu korrigieren. Dies verhindert, dass Maßnahmen abgebrochen werden oder „noch mal ganz von vorne angefangen werden muss“. Beratungskräfte sollten daher regelmäßig und systematisch die Leistungserbringung aller Beteiligten nachhalten. Für das Monitoring sind zwei Voraussetzungen entscheidend:

- Der Hilfeprozess muss ausreichend dokumentiert sein.

- Das Verfahren zum Monitoring muss vereinbart sein, Kontaktdichte und Wiedervorlagen sollten individuell vereinbart werden. Ein Informationsfluss sollte nicht vorausgesetzt, sondern immer verabredet werden.

Der Dokumentation der Hilfeplanung kommt im Rahmen des Monitorings eine besondere Rolle zu, da nicht nur Ziele, Maßnahmen und Fortschritte der Adressatinnen und Adressaten festgehalten und ausgewertet werden, sondern auch „Angebotslücken“, d. h. Ziele, aus denen Angebote abgeleitet werden, die (noch) nicht oder nicht ausreichend vorhanden sind. So kann das einzelfallbezogene Monitoring nicht nur für die einzelfallbezogene Evaluation, sondern auch für das gesamte Case ManagementKonzept und die Netzwerkarbeit genutzt werden. 
6.Stufe: Monitoring

\begin{tabular}{|c|c|c|c|}
\hline Ziel & & blem & \\
\hline $\begin{array}{l}\text { Gew } \\
\text { Infor } \\
\text { über } \\
\text { lauf, } \\
\text { recht } \\
\text { veni } \\
\text { nen. }\end{array}$ & $\begin{array}{l}\text { enz zur } \\
\text { alyse. } \\
\text { kräftiges, } \\
\text { tions- } \\
\text { ifendes } \\
\text { entati- } \\
\text { m, das } \\
\text { edient“ }\end{array}$ & $\begin{array}{l}\text { te Do- } \\
\text { on. } \\
\text { esse } \\
\text { gser- } \\
\text { l. }\end{array}$ & $\begin{array}{l}\text { hes Vor- } \\
\text { sein von } \\
\text { ationen } \\
\text { esentliche } \\
\text { n des Fall- } \\
\text { s. }\end{array}$ \\
\hline \multicolumn{4}{|c|}{ Denkbare Teilschritte } \\
\hline \multicolumn{4}{|c|}{$\begin{array}{l}\text { Beteiligen Sie sich an der Etablierung eines organisationsübergreifenden } \\
\text { Dokumentationssystems. } \\
\text { Sichern Sie Erhebung und Rückfluss von Daten über den Fallverlauf. } \\
\text { Beurteilen Sie die Fortschritte der Adressatin bzw. des Adressaten syste- } \\
\text { matisch und kontinuierlich. } \\
\text { Bewerten Sie - organisationsübergreifend - die Effektivität der einzelnen } \\
\text { Interventionen (u. a. spezielle Hilfsangebote) im Hinblick auf die ge- } \\
\text { wünschten Resultate. } \\
\text { Nutzen Sie aktualisierte Daten, um das Assessment, Zielsetzungen und } \\
\text { den Hilfeplan kontinuierlich zu überprüfen und fortzuschreiben. } \\
\text { Halten Sie die Modifikationen im Assessment, in Zielsetzungen und im } \\
\text { Hilfeplan schriftlich fest. } \\
\text { Beziehen Sie die Adressatin bzw. den Adressaten, ihre bzw. seine Ange- } \\
\text { hörigen und übrige Leistungserbringende in den Auswertungsprozess } \\
\text { ein. } \\
\text { Bringen Sie Ihre Erfahrungen in die fallübergreifenden und -unspezifi- } \\
\text { schen Aktivitäten der Organisation ein (Angebotsplanung und -entwick- } \\
\text { lung etc.). }\end{array}$} \\
\hline
\end{tabular}

\subsubsection{Abschluss und Auswertung (Evaluation)}

Case Management-Prozesse haben ein definiertes und beschriebenes Ende. Dieses ist der (zuvor abgeschätzte) Zeitpunkt der Zielerreichung oder, dass 
die Adressatin bzw. der Adressat in der Lage ist, das Netzwerk von professionellen und ehrenamtlichen Unterstützenden selbstständig zu steuern.

Der Abschluss eines Case Management-Prozesses bedeutet nicht zwingend die komplette Unabhängigkeit von institutioneller oder persönlicher Unterstützung, vielmehr kann die Selbstorganisation benötigter Unterstützung am Ende eines Case Management-Falls stehen. Diese Blickweise ist elementarer Bestandteil von Empowerment, Ziel- und Ressourcenorientierung und damit auch von Case Management und entspricht allen ethischen Standards des Case Managements wie der Sozialen Arbeit. Folgende Tabelle (vgl. Ballew/Mink 1996: S. 270f.; Übersetzung des Autors ${ }^{53}$ ) zeigt die Anzeichen von Adressatinnen und Adressaten für deren Bereitschaft, einen Unterstützungsprozess abzuschließen.

\section{Tabelle 3: Reaktionen von Adressatinnen und Adressaten des Case Manage- ments}

\begin{tabular}{|c|c|c|}
\hline $\begin{array}{l}\text { Anzeichen einer zunehmenden } \\
\text { Kompetenz der Adressatin bzw. } \\
\text { des Adressaten, sich selbst zu } \\
\text { helfen }\end{array}$ & $\begin{array}{l}\text { Anzeichen von zunehmender } \\
\text { Unabhängigkeit }\end{array}$ & $\begin{array}{l}\text { Anzeichen dafür, dass die } \\
\text { Adressatin bzw. der Adressat } \\
\text { das Netzwerk von Unterstützern } \\
\text { und Hilfen effektiv nutzen kann }\end{array}$ \\
\hline $\begin{array}{l}\text { Die Adressatin bzw. } \\
\text { der Adressat... } \\
\text { trifft im Gegensatz zu vorher } \\
\text { wichtige Entscheidungen alleine. } \\
\text { erzählt, dass ihr bzw. inm etwas } \\
\text { gut gelungen ist. } \\
\text { zeigt, dass sie bzw. er kleine } \\
\text { Probleme lösen kann, ohne den } \\
\text { Case Manager zu kontaktieren. } \\
\text { unternimmt ohne Aufforderung } \\
\text { die nächsten Schritte zu einer } \\
\text { Problemlösung. } \\
\text { zeigt sich selbstsicher und } \\
\text { kontrolliert. } \\
\text { macht ohne die Ermunterung } \\
\text { des Case Managers Pläne für die } \\
\text { Zukunft. }\end{array}$ & $\begin{array}{l}\text { Die Adressatin bzw. } \\
\text { der Adressat... } \\
\text { zieht es vor, mit dem Case } \\
\text { Manager zu telefonieren anstatt } \\
\text { sich zu treffen oder wünscht } \\
\text { monatliche statt wöchentliche } \\
\text { Termine; verpasst oder vergisst } \\
\text { Verabredungen, wenn alles gut } \\
\text { läuft. } \\
\text { kommt mehrere Wochen ohne } \\
\text { den Case Manager zurecht. } \\
\text { entwickelt zu einer Person der } \\
\text { unterstützenden Dienste engere } \\
\text { Kontakte als zum Case Manager. }\end{array}$ & $\begin{array}{l}\text { Die Adressatin bzw. } \\
\text { der Adressat... } \\
\text { wird, wenn nötig, von ihrem bzw. } \\
\text { seinem persönlichen Netzwerk } \\
\text { unterstützt. } \\
\text { trifft, falls notwendig, ohne die } \\
\text { Hilfe des Case Managers } \\
\text { Verabredungen mit beteiligten } \\
\text { Diensten. } \\
\text { kann selbständig den Kontakt } \\
\text { zum Netzwerk bzw. einzelnen } \\
\text { Beteiligten halten. } \\
\text { hat einen besseren Überblick } \\
\text { über die Angebotsstruktur und } \\
\text { mögliche Hilfen. }\end{array}$ \\
\hline
\end{tabular}

53 Um die Authentizität zu wahren, wurde in der Übersetzung darauf verzichtet, eine gendergerechte Sprache zu verwenden. 
Der Abschluss des Case Managements beinhaltet die Auswertung (Evaluation) des Gesamtprozesses. Grundsätzlich gehören die Bewertung der eigenen Arbeit und ihre Überprüfung auf Effektivität und Effizienz sowie die Suche nach Verbesserungspotenzial zu einer professionellen Arbeitshaltung. Im Case Management geht es in einer abschließenden Auswertung aber auch um die Verknüpfung zwischen der Reflexion der Einzelfallarbeit und der Weiterentwicklung der Angebotsstruktur.

Evaluiert werden sollten folgende Schwerpunkte (vgl. van Riet/Wouters 2002: S. 240):

- Der Hilfeplan in Bezug auf die erreichten Ziele und Resultate.

- Die Arbeitsmethoden und die Zusammenarbeit mit Dritten.

- Die Zufriedenheit der Zielgruppe.

Eine Auswertung von Case Management-Prozessen setzt Dokumentation voraus, z. B., um die Zielerreichung zu überprüfen oder den ursprünglichen Zustand beim Assessment mit der jetzigen Situation zu vergleichen. Zum zweiten Punkt gehören die Reflexion der eigenen Arbeit und eine Dokumentation des Aufwands für einen Fall. Hinzu kommt die systematische Nachfrage bei den beteiligten Kooperationspartnerinnen und -partnern zu deren Einschätzung bezüglich der Zielerreichung und der Effektivität sowie Effizienz der Hilfen. Hierzu gehört auch, Kooperationsbeteiligten sowohl positive als auch negative Rückmeldungen über ihre Hilfeleistungen zu geben. Zentral für die Beurteilung des Erfolgs eines Unterstützungsprozesses ist die Einschätzung der adressierten Personen. Ihre Meinung sollte umfassend erhoben und in jedem Fall in einem abschließenden Gespräch behandelt werden.

Alle Auswertungen auf Einzelfallebene sollten in der Auswertung (Evaluation) der gesamten Organisation zusammengefasst werden. Die Summe der Einzelbewertungen ergibt eine Bewertung der Gesamtheit und fördert so auch Lücken im System zutage. Systematisch zusammengefügte Auswertungen von Case Management-Prozessen ergeben Hinweise für die Planung und Gestaltung der Kooperationsbeziehungen sowie für die (politischen) Forderungen an die Angebotsstruktur.

\subsection{Interaktion im Rahmen des Case Managements}

Case Management bedeutet explizit nicht, die in Abschnitt 5.4 dargestellten Abläufe technisch möglichst perfekt zu absolvieren, vielmehr ist es in all seinen Varianten eine spezifische Form professioneller Intervention in 
Lebensverhältnisse. Was dies bedeutet und beinhaltet, soll in diesem Kapitel diskutiert werden.

Im Konzept des Case Managements spielt die zielgerichtete, aber gleichwohl themenoffene Beratungsarbeit mit den Adressatinnen und Adressaten der sozialen Dienstleistung eine herausragende Rolle. Auf der Ebene der Intervention ist Case Management demnach unter der Perspektive der sozialwissenschaftlichen Diskussion zur „Dienstleistungsarbeit“ zu sehen, in der vor allem die Bedeutung der Kooperation bzw. „Ko-Produktion“ für die Erbringung personenbezogener Dienstleistungen in den Vordergrund gerückt wurde. „Der Kunde oder Klient muss sich - aktiv oder passiv - am Produktionsprozess beteiligen, er muss mitmachen. Dieses Mitmachen reicht vom Stillhalten beim Friseur (...) bis hin zu Dienstleistungen, in denen der Klient praktisch die Hauptarbeit übernehmen muss und der Produzent in die Rolle des mehr oder weniger passiven Zuhörers und Deuters gerät“ (Badura/Gross 1976: S. 69). Zustandekommen und Qualität der Dienstleistung hängen deshalb in hohem Maße von der persönlichen Interaktion zwischen „Leistungsanbieter" und „Kundin“ bzw. „Kunde“ ab: „Das Aufeinandertreffen von Produzent und Kunde im Gespräch, im Umgang in der face-to-face-Beziehung, weckt schlagartig Facetten der zwischenmenschlichen Beziehung, die in der industriellen Sachgüterproduktion mit getrennter Produktion und Konsumation verschwinden mussten: Unmittelbarkeit, gegenseitige Rücksichtnahme, Leiblichkeit und Gefühle, aber auch wechselseitige Kontrolle“ (ebd.: S. 69f.). Das Ergebnis der Dienstleistung ist damit bedingt durch die Kooperationsfähigkeit und -willigkeit ihrer Adressatinnen und Adressaten; die Konsumentin bzw. der Konsument ist nicht nur "Nachfragende“ bzw. „Nachfrager“, sondern gleichzeitig Teil des Produktionsprozesses der Leistung: „Input und Kooperation/Nicht-Kooperation von Seiten des Konsumenten beeinflussen die Qualität der Gesamtleistung beziehungsweise sie wird überhaupt erst zu einer Leistung durch die Zusammenarbeit" (Oppen 1995: S. 22). Personenbezogene Dienstleistungen werden im Rahmen eines spezifischen „Arbeitsbündnisses" erbracht, das die handelnden Personen eingehen. Dabei geht es um ein kontextgebundenes „Aushandeln“ dessen, was aktuell und künftig geschehen soll, als Festlegung der Regeln, die in der je spezifischen Interaktionssituation gelten sollen. Dieses „Arbeitsbündnis“ baut auf institutionellen Strukturen auf, stiftet aber dennoch ein je spezifisches Verhältnis zwischen konkret interagierenden Personen - seine "Tragfähigkeit" muss sich im weiteren Interaktionsprozess erweisen.

Vor diesem Hintergrund wird die hohe Bedeutung der persönlichen Interaktion für das Gelingen des Case Managements deutlich. Und hier ist es 
insbesondere die Art der Gesprächsführung, die die Interaktion prägt. In einem Forschungsprojekt zum Case Management im Kontext des SGB XII wurde versucht, die Varianz von Gesprächssituationen in einer kleinen Typologie abzubilden, die auch auf andere Arbeitsfelder des Case Managements übertragen werden kann (vgl. Reis/Schu/Brülle 2009). In zwei Sozialämtern wurden insgesamt 17 Gespräche beobachtet und detailliert protokolliert. Davon waren acht Erstgespräche und neun Folgegespräche, die mithilfe einer qualitativen Inhaltsanalyse im Hinblick auf die Art der Gesprächsführung ausgewertet wurden. Der Beratungsstil der Fallmanager ${ }^{54}$ bildete die Grundlage für eine kleine Typologie, die im Folgenden dargestellt wird. Generell handelt es sich um Beratungen mit „diffusem Problembezug", d. h. die Beratungsthemen stehen nicht vorab fest (z. B. als Rechtsberatung, Schuldnerberatung etc.), sondern werden erst im Gesprächsverlauf entwickelt. Die Art und Weise dieser Entwicklung und weiteren Bearbeitung kann dabei differenziert im Hinblick darauf betrachtet werden, ob es sich um ein „offenes“, d. h. nur gering strukturiertes, um ein über die Assessment-Kategorien stark vorstrukturiertes oder - als „Mittelweg" - um ein zwar offenes, aber von den Fallmanagern im Verlauf gesteuertes Gespräch handelt.

Insgesamt konnten vier unterschiedliche Typen von Gesprächen identifiziert werden:

- offene Gespräche (5.5.1);

- Gespräche, in denen die Fallmanager eine deutlich steuernde Funktion übernehmen (5.5.2);

- Gespräche, in denen die Fallmanager im Wesentlichen die (vorgegebenen) Assessment-Kategorien „abfragen“ (5.5.3);

- Gespräche, in denen kein Arbeitsbündnis zu Stande kam (5.5.4).

\subsubsection{Offene Gespräche}

Dieser Typus von Gesprächen, dem acht von 17 Fällen zugeordnet werden konnten, lässt sich dadurch kennzeichnen, dass die Fallmanager versuchen, durch Gesprächsführung, aber auch Körperhaltung (z. B. Blickkontakt), eine offene Gesprächsatmosphäre zu schaffen, in der den adressierten Personen Gelegenheit gegeben wird, ihre aktuelle Lebenssituation und ihre Problemlage ausführlich zu schildern. Die Fallmanager nehmen

54 In diesem Kapitel wird von „Fallmanagern“ gesprochen, weil das dem Sprachgebrauch der beforschten Praxis entspricht. 
dabei keine oder nur eine geringe steuernde Funktion wahr, sondern legen Wert auf die Exploration der Gesamtsituation. ${ }^{55}$

Beispiel 1

Das Verhalten der Fallmanagerin ist freundlich, empathisch, akzeptierend, bei Bedarf nach- und rückfragend und dadurch etwas richtungssteuernd; sie muss größtenteils lediglich Stichworte vorgeben, denn der Adressat ist sehr redselig und spricht viele Themenbereiche bereits von sich aus an; die Fallmanagerin ist sehr souverän und sicher in ibrem Vorgehen und wirkt dadurch vertrauenerweckend. Der Adressat ist sehr offen und freundlich, wirkt extrovertiert, ist sehr auskunftsfreudig bzw. mitteilungsbedürftig, greift gegebene Stichworte auf und erzählt bereitwillig und recht ausführlich, springt aber gelegentlich innerhalb eines Themas noch einmal zu einem anderen zurück oder greift Themenbereichen vor, er wirkt insgesamt sehr interessiert und extrem motiviert. Er betont durchgängig, dass er sehr gern wieder eine Beschäftigung hätte. Die Gesprächssituation wirkt entspannt, die Gesprächspartner scheinen sich sympathisch zu sein, das Gespräch ist ständig im Fluss, es gibt keinerlei Verständigungsschwierigkeiten.

Wie der folgende Protokollausschnitt zeigt, können nicht nur Erst-, sondern auch Folgegespräche von der Strategie bestimmt sein, durch die Art der Kommunikation Vertrauen zu schaffen und aufrecht zu erhalten, d. h. ein Arbeitsbündnis aufzubauen. Da in den Folgegesprächen meist kein Assessment mehr durchgeführt wird, dominiert der offene, unstrukturierte Charakter dieser Art der Gesprächsführung.

Beispiel 2

Das Gespräch wird seitens der Beraterin frei geführt, es erfolgt keine Orientierung am Assessmentbogen. Teilweise nimmt es die Form einer informellen Plauderei an. Die Fallmanagerin zeigt sich verständnisvoll und räumt der Adressatin viel Raum für ibre Ausfübrungen ein, so dass vor allem zu Beginn des Gesprächs eher die Adressatin selbiges dominiert und die angesprochenen Themen bestimmt. Die Beraterin stellt in der ersten Gesprächshälfte nur wenige Zwischenfragen oder äußert nur einige Kommentare. Die Adressatin ist sehr gesprächig und der Fallmanagerin gegenüber sehr offen. Sie beantwortet alle Fragen der Fallmanagerin ausfübrlich und erzählt auch ohne Aufforderung viel von sich. Man hat den Eindruck, sie nutzt diesen Termin als Gelegenheit, sich zu unter-

55 Die im Folgenden kursiv gesetzten Textstellen sind im Wesentlichen dem Beobachtungsprotokoll eines Mitglieds des damaligen Forschungsteams entnommen, wobei geringfügige Änderungen im Sinne der Einheitlichkeit dieses Leitfadens vorgenommen wurden. 
halten. Allerdings kommt sie bei ihren Ausführungen immer wieder auf das Fehlen eines Tagesrhythmus' zurück. Ihrem Leben einen geregelten Tagesablauf zu geben, scheint ihr wichtigstes Anliegen zu sein. Die Abstimmung zwischen Fallmanagerin und Adressatin ist insofern sebr gut, als dass die Adressatin das Angebot der Beraterin begeistert annimmt und sich spontan zu einem Besuch der vorgestellten Einrichtung entscheidet. Die Interaktion gestaltet sich eher locker.

Diese Art der Gesprächsführung scheint gut geeignet zu sein, um auch eher verschlossene Adressatinnen und Adressaten „zu öffnen“, sie aus der kommunikativen Isolation, in der sie sich häufig befinden, herauszulocken. Die folgende Protokollnotiz macht deutlich, dass die Fallmanager dabei sehr behutsam vorgehen müssen und immer wieder signalisieren, dass es die Adressatinnen bzw. Adressaten selbst in der Hand haben, ob und worüber gesprochen wird.

\section{Beispiel 3}

Die Fallmanagerin tastet sich aufgrund der Vorgeschichte der Adressatin sehr vorsichtig an diese heran. Sie weist die Adressatin immer wieder darauf hin, dass sie das Gespräch jederzeit abbrechen könne, wenn es zu viel werde. Die Fallmanagerin ist durchweg freundlich und sowohl sehr verständnisvoll als auch mitfühlend gegenüber der Adressatin. Sie lächelt die Adressatin oft an und nickt ibr zu. Sie wählt ibre Fragen sehr besonnen, um der Adressatin nicht zu nabe zu treten und sagt ihr mehrfach, dass sie auf Fragen nicht antworten müsse, wenn sie dies nicht wolle. Sie erklärt der Adressatin den Sinn und Zweck des Fallmanagements und überlässt ibr die Entscheidung, ob sie daran teilnehmen möchte oder nicht. Die Fallmanagerin wendet sich der Adressatin während des gesamten Gesprächs mit dem Körper zu, außer wenn sie etwas in den PC eingibt. Den Blickkontakt hält die Fallmanagerin aber nicht durchgehend zur Adressatin, da diese auch oft einfach im Raum herumschaut. Die Fallmanagerin versucht der Adressatin durchgehend zu vermitteln, dass nichts gegen den Willen der Adressatin stattfinden werde und man ibr nur helfen wolle. Sie lobt die Adressatin zum Ende des Gesprächs für ibren Mut und bedankt sich für die Offenheit. Die Adressatin ist zu Beginn des Gesprächs sehr schüchtern und verunsichert. Sie beantwortet nur die Fragen der Fallmanagerin, von sich selbst aus erzäblt die Adressatin nicht viel. Sie begründet ihre Verschlossenheit mit ihrer Angst vor Menschen. Zwischendurch beginnt die Adressatin immer wieder zu weinen, hört aber nach kurzer Zeit auch wieder auf. Durch die einfühlsame Art der Fallmanagerin scheint die Adressatin immer mehr Vertrauen zu gewinnen; sie wird immer ruhiger.

Diese Gespräche haben für einige Adressatinnen und Adressaten erkennbar eine therapeutische Funktion. Vor allem diejenigen, die persönlich 
stark isoliert leben, nutzen die Kommunikation mit den Fallmanagern, um fehlende soziale Kontakte wenigstens punktuell zu ersetzen. Hierüber kann eine starke Bindung entstehen, gleichzeitig kann die Art der Kommunikation dazu führen, dass die Aufgabe des Fallmanagements, Leistungen Dritter zu initiieren und zu steuern, in den Hintergrund tritt.

\section{Beispiel 4}

Die Fallmanagerin gewährt der Adressatin viel Raum für ibre Ausführungen und fragt an verschiedenen Stellen mehrfach nach, ob es noch etwas gäbe, das der Adressatin am Herzen läge und über das sie reden möchte. Sie bietet der Adressatin sowobl emotionale als auch praktische Unterstützung bei den geschilderten Problemen. Die Adressatin ist sehr gesprächig und beantwortet alle Fragen ausfübrlich und erzählt auch ohne Aufforderung viel von sich. Dies wird vor allem an den Stellen im Gespräch deutlich, an denen die Fallmanagerin fragt, ob es noch etwas gäbe, über das sie sich unterhalten möchte. Die Adressatin überlegt jedes Mal kurz und beginnt anschließend über verschiedene Begebenheiten zu berichten. Die Interaktion ist gut und das Gespräch fließend. Die Adressatin ist sehr offenherzig und antwortet ausführlich. Die Fallmanagerin lässt der Adressatin viel Raum für ihre Ausführungen, geht auf die angesprochenen Themen der Adressatin ein und kommentiert diese meist verständnisvoll, zum Teil auch lobend. Sie bietet der Adressatin diverse Unterstützung an (Anruf beim Tierheim, Internetrecherche für Hilfsangebote für betroffene Angehörige, Begleitung zur Caritas, finanzieller Vorschuss für eine neue Brille), die diese größtenteils annimmt. Vor allem die Möglichkeit eines Darlehens stößt bei ihr auf großes Interesse.

Es ist erstaunlich, dass es den Fallmanagern in fast der Hälfte aller beobachteten Gespräche gelingt, eine vertrauensvolle Gesprächsbeziehung zu ihren adressierten Personen aufzubauen und damit ein Arbeitsbündnis zu schaffen, das auch über einen längeren Zeitraum tragfähig bleibt.

\subsubsection{Gespräche, in denen die Fallmanager eine steuernde Funktion übernehmen}

Im Gegensatz zum erstgenannten Typus zeichnen sich die vier Gespräche dieses Typs dadurch aus, dass die Fallmanager zwar ebenfalls versuchen, eine für die Adressatinnen und Adressaten angenehme Gesprächsatmosphäre zu schaffen, gleichzeitig aber das Gespräch stärker strukturieren. Hierzu gehört auch die Strategie, (zumindest) zunächst nicht in die Tiefe zu gehen. 
Beispiel 5

Die Fallmanagerin zeigt sich gegenüber dem Ehepaar mit Kind freundlich, interessiert, verständnis- und rücksichtsvoll. Sie erklärt ausführlich Ziel und Funktion des Fallmanagements und weist mehrfach darauf hin, dass sie ibnen helfen bzw. gemeinsam mit ihnen Lösungsmöglichkeiten für Probleme erarbeiten möchte. Sie geht auf die Adressatin und den Adressaten ein, hört ibnen aufmerksam zu und fragt meist gezielt nach. Nur an wenigen Stellen blockt sie das Gespräch offen ab mit dem Hinweis, an dieser Stelle nicht zu sehr in die Tiefe gehen zu wollen. Dies wird von der Adressatin und dem Adressaten akzeptiert und hat keinen Einfluss auf seine Offenheit im weiteren Gesprächsverlauf. Sie berücksichtigt insbesondere die Zurückhaltung der Frau und erspart ihr die Thematisierung ibrer Suchtkarriere und Krankheitsgeschichte in diesem ersten Gespräch. Die Fallmanagerin versucht zudem, ermutigend auf die Adressatin einzuwirken, als diese Selbstzweifel äußert. Auch bedankt sie sich bei Beiden für ibre Offenheit und das Vertrauen, das ibr im Gespräch entgegengebracht wird. Die Fallmanagerin zeigt Interesse an dem anwesenden Kleinkind des Paars und Verständnis für sein „quengeliges Verhalten“. Sie nimmt im Gesprächsverlauf auch mehrfach Kontakt zu dem Kind auf, was sichtlich zur Auflockerung der Atmosphäre beiträgt.

$\mathrm{Zu}$ diesem Typus gehören auch Gespräche, in denen die Fallmanager die Adressatinnen und Adressaten in Folgegesprächen sehr zielstrebig mit ihren Problemen und möglichen Lösungsansätzen konfrontieren - immer aber auf der Basis einer offenbar stabilen Beratungsbeziehung.

Beispiel 6

Die Fallmanagerin ist dem Adressaten gegenüber sehr offen und hört interessiert $z u$. Sie geht auf dessen Schilderungen ein. Bei Problemen des Adressaten, wie z. B. dem Arztbesuch, zeigt sie Lösungswege auf und versucht ibn freundlich in die "richtige" Richtung zu bringen. Insbesondere in Bezug auf die Alkoholsucht des Adressaten ist die Fallmanagerin sehr beharrlich und macht ibm deutlich, dass er sich damit auseinandersetzen muss. Das Gespräch wird eindeutig von ihr gesteuert. Sie stellt die Fragen und hakt bei Bedarf nach. Vorschläge und Ideen unterbreitet sie mit Erklärungen, macht aber auch deutlich, dass sie die Durchführung für wichtig und sinnvoll halte. Der Adressat scheint während des gesamten Gesprächs etwas nervös zu sein. Auf Nachfragen der Fallmanagerin erzäblt er offen und auch ausgiebig. Er ist sehr reflektiert und äußert sich ehrlich über seine Problematik. Er scheint sehr frob darüber, dass die Fallmanagerin auf seine Wünsche nach einer Arbeitserprobung eingeht, da deutlich zu erkennen ist, dass er nicht mehr länger abwarten, sondern endlich handeln möchte. Das Erstellen 
einer Hilfeplanung mit Festlegung von Zielen erfolgt in gemeinsamer Absprache und obne Druck.

Die folgende Protokollnotiz macht deutlich, dass die Fallmanager mithilfe einer solchen Strategie eine wichtige orientierende Funktion für die Adressatinnen und Adressaten übernehmen können.

\section{Beispiel 7}

Die Fallmanagerin macht durchgehend einen sehr souveränen und gleichzeitig akzeptierenden Eindruck. Sie erscheint sehr kompetent, motivierend, engagiert und empathisch. Sie steuert das Gespräch und versteht es, den Adressaten durch Rückfragen zum kritischen Reflektieren seiner Vorstellungen bzw. getroffener Entscheidungen zu bewegen. Sie ist durchweg dem Adressaten gegenüber sebr freundlich. Der Adressat scheint der Fallmanagerin bereits großes Vertrauen entgegenzubringen; er wirkt sehr offen, mitteilungsbedürftig und legt scheinbar großen Wert auf ihre Meinung bzw. positive Rückmeldung.

Alle hier beobachteten Gespräche dieses Typus zeigen, dass sich die Fallmanager auf einem „sicheren Terrain“ bewegen, d. h. sie schaffen es, zu strukturieren, ohne dass die Basis einer vertrauensvollen Ko-Produktion mit ihren adressierten Personen gefährdet wäre. Dies unterscheidet sie deutlich vom nächsten Typus.

\subsection{3 „Abfragen“ im Rahmen des Assessments}

Diesem Typus sind drei Gespräche zuzuordnen. Sie zeichnen sich dadurch aus, dass die Fallmanager bemüht sind, den Assessment-Bogen „abzuarbeiten“, d. h. alle dort aufgeführten Fragen zu stellen und die Antworten zu notieren - ohne in die Tiefe zu gehen, selbst wenn sich im Gespräch hierzu Anhaltspunkte ergeben. Dies führt dazu, dass Adressatinnen und Adressaten, die mit anderen Erwartungen gekommen sind, im Gesprächsverlauf immer verschlossener werden. Damit wird das Gegenteil dessen erreicht, worauf das Assessment eigentlich abzielen sollte.

Beispiel 8

Die Fallmanagerin erscheint dem Adressaten gegenüber unkonzentriert. Manche Themen, z. B. soziale Kontakte, greift sie mit der stets gleichen Frage wiederholt auf, allerdings jedes Mal nur sebr oberflächlich, worauf der Adressat stets die gleiche Antwort gibt. In die Tiefe geht sie nicht, stellt selten Fragen nach Gründen für Situationen oder Verhalten und auch nicht nach den Auswirkungen für 
den Adressaten. Sie geht - besonders bei entscheidenden Informationen bzw. eventuellen Schlüsselerlebnissen, die der Adressat erwähnt - wenig analytisch vor. Sie erscheint insgesamt eher wenig empathisch. Ihr Verhalten könnte auf den Adressaten desinteressiert und unaufmerksam wirken, sie scheint ihm gegenüber eher reserviert. Der Adressat macht zu Beginn einen offenen, aufgeschlossenen, freundlichen Eindruck, erscheint erwartungsvoll; zum Ende hin macht er den Eindruck, als habe sich die Verständnislosigkeit der Fallmanagerin auf ibn übertragen, da seine Erwartungen nicht erfüllt wurden.

Auch wenn die Haltung der Fallmanager offen und das Bemühen spürbar ist, eine produktive Gesprächsatmosphäre herzustellen, wirkt der Rekurs auf das „Abarbeiten“ vorgegebener Fragen negativ auf den Gesprächsverlauf ein, wie das folgende Beispiel zeigt.

Beispiel 9

Die Beraterin ist dem Adressaten gegenüber sehr freundlich. Sie bietet ihm zu Beginn des Gesprächs einen Kaffee an und einen weiteren Kaffee im Gesprächsverlauf. Sie eröffnet das Gespräch mit eher allgemeinen Fragen („Was haben Sie bei dem schönen Wetter gemacht?"). Das Gespräch bekommt dadurch den informellen Charakter einer Plauderei. Dies trägt zur Auflockerung der Situation bei und erleichtert dem Adressaten den Einstieg in das Gespräch. Allerdings wird zu dessen Beginn nicht deutlich formuliert, was das Ziel des Termins ist. Erst nach einiger Zeit greift die Beraterin eine Aussage des Adressaten bezüglich seiner Einsamkeit auf und erklärt, dass der Aufbau von sozialen Kontakten das Ziel sein solle und dass sie außerdem das in der letzten Sitzung begonnene Assessment zu Ende führen möchte. Im Folgenden hält sich die Beraterin eng an die Fragen des Assessments und arbeitet diese nacheinander ab. Dabei liest sie die Fragen oftmals vom Bildschirm ab. Dies klingt, als würde sie sich die Fragen selbst vorlesen und müsse sich erst darüber klar werden, was diese eigentlich bedeuten. Nachdem sie eine Frage abgelesen bzw. vor sich hingesprochen hat, versucht sie diese auf den vorliegenden Fall zu beziehen und richtet die Frage dann meist noch mal gezielt an den Adressaten. Die Fallmanagerin stell häufig Nachfragen, was den Eindruck erweckt, als hätte sie nicht aufmerksam zugehört. Einige Sachverhalte zur gesundheitlichen Situation erfragt sie mehrfach. Die Fragen wirken überflüssig, da der Adressat die Antworten bereits im Gesprächsverlauf geliefert hat. Obwohl die Fallmanagerin nicht desinteressiert wirkt, häufig nachfragt, versucht zu motivieren und dem Adressaten viel Zeit für seine Ausfübrungen zugesteht, bleiben einige vom Adressaten erwähnte, wichtige Ereignisse von der Fallmanagerin unkommentiert. 
Die hier diskutierten Gespräche führen zwar nicht direkt zum Scheitern der Arbeitsbündnisse, dennoch ist zu befürchten, dass es hierhin kein weiter Weg mehr ist. Die Erwartungen der Adressatinnen und Adressaten sind enttäuscht, Vertrauen wird eher ab- als aufgebaut, eine Basis für die Bearbeitung „sensibler“ Themen steht nicht in Aussicht.

\subsubsection{Scheiternde Arbeitsbündnisse}

In nur zwei von 17 Gesprächen wird deutlich, dass kein tragfähiges Arbeitsbündnis zu Stande kommt. In einem Fall liegt dies am Misstrauen des Adressaten.

Beispiel 10

In diesem Gespräch macht die Fallmanagerin durchgehend einen sehr starken und akzeptierenden Eindruck; sie erscheint sehr kompetent/fachkundig, souverän, aufmerksam und empathisch. Sie steuert das Gespräch, hakt nach und versucht durchweg einfühlend, beim Adressaten Vertrauen zu erwecken. Sie ist ibm gegenüber sehr freundlich. Wie in einem anderen Gespräch versucht sie, den Adressaten dazu zu bringen, über die Möglichkeiten zu reflektieren. Der Adressat ist zwar freundlich, aber fast durchgängig ablehnend, eher misstrauisch und verschlossen. Er scheint von Beginn an das Fallmanagement abzulehnen. Zeitweise wirkt er etwas eingeschüchtert (von der gesamten Situation, nicht von der Fallmanagerin). Die Fallmanagerin sieht teilweise einen Bedarf an Fallmanagement, der Adressat jedoch nicht. Als deutlich wird, dass der Adressat das Fallmanagement ablehnt, gibt ihm die Fallmanagerin zu verstehen, dass sie seinen Wunsch akzeptiert, bietet aber an, dass er sich bei Bedarf jederzeit melden könne.

Im zweiten Gespräch scheint eher die Haltung der Fallmanagerin gegenüber dem Adressaten verantwortlich zu sein für eine gestörte Kommunikation.

\section{Beispiel 11}

Vor Beginn des Gesprächs mit dem Adressaten vermittelt die Fallmanagerin einen unmotivierten und gelangweilten Eindruck. Während des Gesprächs ist sie zwar freundlich, gibt dem Adressaten positives Feedback und es könnte der Eindruck entstehen, dass sie eine akzeptierende Haltung einnimmt. Sie wirkt allerdings etwas planlos/unstrukturiert in ihrem Vorgehen, äußert gelegentlich ibre Meinung, versucht aber nicht, den Adressaten dazu zu bringen, selbst zu reflek- 
tieren. Sobald der Adressat aus dem Raum ist, öfnet sie sogleich hektisch das Fenster mit den Worten „puh, der hat aber gestunken!“. Außerdem wird klar, dass sie keineswegs eine akzeptierende Haltung einnimmt und ibr - die Eigeninitiative des Adressaten lobendes - Feedback nicht ernst gemeint war, sondern sie dieses als Aufbau einer Scheinwelt einschätzt: „Wenn sich jemand auf ihn einlassen würde, dann doch auch bloß jemand wie er selbst, eine Obdachlose oder so", "sobald die bei Edeka den sehen, ist ja schon klar, dass die den nicht nehmen." Es scheint, als fehle ihr manchmal tatsächlich das Verständnis im Sinne dessen, dass sie mit der Lebenswelt des Adressaten nichts anfangen kann.

Obwohl im SGB XII die Gespräche grundsätzlich freiwillig sind, kann der Fall eintreten, dass ein Arbeitsbündnis nicht zu Stande kommt oder früh scheitert. Allerdings trifft dies nur für zwei der insgesamt 17 Gespräche zu, die an beiden Standorten beobachtet wurden - ein Indiz für eine eher vertrauensvolle Gesprächsatmosphäre.

\subsubsection{Fazit}

In der Interaktion im Case Management zeigt sich, dass Ko-Produktion die zentrale Voraussetzung einer personenbezogenen Dienstleistung darstellt. Damit tritt nicht nur aus pädagogischer Warte die Berücksichtigung der Adressatinnen und Adressaten als mündige Kooperationspartnerinnen und -partner ins Zentrum. Darüber hinaus liegt erst in der Herstellung eines Arbeitsbündnisses überhaupt die Chance, Bedarfe der Adressatinnen und Adressaten erkennen zu können. Wenn sich die Beratungsarbeit primär an den Ressourcen der Adressatinnen und Adressaten orientiert, ist dies sowohl Ausdruck des Respekts vor den individuellen Bedarfslagen als auch dem Umstand geschuldet, dass sie nur auf diese Weise erfolgreich sein kann. Allerdings darf nicht vergessen werden, dass die Bedingungen und Perspektiven, unter denen die Beteiligten in den Beratungsprozess eintreten, grundsätzlich verschieden sind und bleiben. Wesentliches Merkmal für die Herstellung und Kultivierung eines tragfähigen Arbeitsbündnisses bleibt es daher auch, offen mit der Asymmetrie zwischen den Beteiligten umzugehen, die eigene Rolle darin zu veranschaulichen und von dort aus nach gemeinsamen Aushandlungsprozessen zu suchen, in denen die Lebenssituation der Adressatinnen und Adressaten angemessen berücksichtigt werden kann. Alles andere würde die Grundstruktur verschleiern und die Gegenüber als Personen nicht ernst nehmen. 


\subsection{Ausblick}

Das Handlungskonzept „Case Management“ birgt für die Praxis viel Gestaltungsspielraum, der produktiv genutzt werden kann, um „rechtskreisübergreifendes Case Management“ zu etablieren. Gleichzeitig treten alte Widersprüche im neuen Gewand auf, die die Soziale Arbeit von jeher prägen („doppeltes Mandat“) und ziehen Beratungsdilemmata nach sich, die typisch für die Leistungsprozesse in der Sozialen Arbeit sind. Um innerhalb dieser Struktur wenigstens ein Mindestmaß an Klärung für Professionelle sowie Adressatinnen und Adressaten herbeiführen zu können und nicht restlos im Ungewissen über die eigene Arbeit zu bleiben, bedarf es unterschiedlicher Orientierungsparameter:

- Ausgangspunkt für die Ausgestaltung des Handlungskonzepts ist die Formulierung einer eindeutigen Leitidee. Für die anschließende Praxis ist ein wesentliches Merkmal die eindeutige Entscheidung bzw. perspektivische Ausrichtung dessen, was Case Management im jeweiligen Arrangement sein und tun soll. Daran bemisst sich bereits, welche der professionellen Kompetenzen vor Ort voraussichtlich betont werden. Liegt der Schwerpunkt primär auf der „Vermittlung“ an Dritte, sind Kenntnisse über das Leistungsangebot und die Fähigkeit des „Matchings“ stärker gefragt. Werden dagegen lebensweltliche Fragen programmatisch in den Vordergrund gerückt, stehen mehr pädagogisch begleitende Kompetenzen im Fokus. Im ersten Fall wird wie selbstverständlich von einem Arbeitsbündnis ausgegangen, d. h. es wird unterstellt, dass die Beteiligten sich über den grundsätzlichen Sachverhalt einig sind und lediglich den Weg klären, um das gewünschte Ziel zu erreichen. Systematisch betrachtet ist die Herstellung dieses Konsens' aber bereits ein wesentlicher Teil des Beratungsprozesses. Angesichts der häufig diffusen Problemlagen der in Frage kommenden Zielgruppe kann in der Praxis nicht auf eine intensive persönliche Hilfe verzichtet werden.

- Case Management kann angesichts der Komplexität der Arbeitsaufträge sowie der Verantwortung, Adressatinnen und Adressaten „aus einer Hand“ zu unterstützen, keine „einsame Tätigkeit“ sein. Deshalb sind kollegiale Formen gegenseitiger Unterstützung (Fallkonferenzen, kollegiale Fallberatung) ebenso wichtig wie der Blick von außen (Praxisreflexion, Supervision). Dadurch können sowohl übergreifende fachliche Standards in die Praxis hineingetragen und für den spezifischen Kontext nutzbar gemacht als auch „einfach“ fallbezogene Fragen abgestimmt werden. Darüber hinaus können strukturelle Überforderungen 
früh erkannt und ggf. vermieden werden. Nicht zuletzt bieten sie einen Schutz für die adressierten Personen. Die hohe Entscheidungskompetenz, gepaart mit den zumeist in der Praxis auftretenden Unsicherheiten, kann ansonsten zur Folge haben, dass Entscheidungen nicht in Bezug zu den Bedarfslagen der Adressatinnen und Adressaten gefällt werden. Die Verunsicherung kann zum Rückgriff auf Routinen führen, die mit den veränderten Anforderungen nicht übereinstimmen und negative Beratungswirkungen nach sich ziehen. Dennoch: Um angemessen individuelle und sozialräumliche Ressourcen berücksichtigen zu können, braucht es hohe Entscheidungskompetenzen für die Case Manager, um mit dem vorhandenen Angebot arbeiten zu können, bzw. fehlende Angebote zu schaffen und damit fallübergreifend steuernd agieren zu können. Um im Case Management professionell zu arbeiten, bedarf es, je nach Auslegung des Konzepts, unterschiedlicher Kompetenzen und Wissensarten. Dazu zählen sowohl die Kenntnis rechtlicher Rahmenbedingungen als auch das Wissen über den Sozialraum. Im Zentrum stehen Beratungskompetenzen und -erfahrungen. Die Klammer aus den verschiedenen Ebenen der Tätigkeit schließlich bildet die Fähigkeit, dies im Kontext eines Falls und darüber hinaus „managen“, also koordinieren und organisieren zu können. Um dieser Vielfalt von Anforderungen gerecht werden zu können, sind Personalentwicklung und Fortbildung dringend erforderlich, denn Case Management ist nicht nur die Summe einzelner Kompetenzen, sondern es offenbart sich darin eine Veränderung professioneller Standards von sozialer Dienstleistung.

- Unterhalb der Formulierung einer Leitidee bestimmen die organisatorischen Umsetzungspraktiken das operative Geschäft entscheidend mit. Unterschiedliche organisatorische Umsetzungen werden für Unterschiede in der Nutzung der Gestaltungsspielräume sorgen und somit verschiedene Organisationskulturen hervorbringen. Für das Professionsprofil "Case Management" sind die organisatorischen Bedingungen besonders deshalb wichtig, weil das Konzept zentral auf der Idee der Vernetzung aufbaut. Wesentlich ist, unabhängig vom Aufbau der Organisation vor Ort, dass nicht nur alle Beteiligten die Rolle des Case Managements zur Kenntnis nehmen und formal absegnen, sondern sie von den politisch Verantwortlichen bis zu den Adressatinnen und Adressaten anerkannt und begleitet wird. Ansonsten laufen Maßnahmen ins Leere, Absprachen werden unverbindlich und Adressatinnen und Adressaten werden zwischen den verschiedenen Akteuren hin und her verwiesen. Was auf der programmatischen Ebene die eindeutigen 
Grundentscheidungen sind, übersetzt sich in die Behörden und Trägerstrukturen in Form von verbindlicher Zuständigkeit. Es gilt - möglichst in „Produktionsnetzwerken“ - Verfahrenswege zu definieren und Verantwortlichkeiten festzulegen. Schließlich sind für eine sinnvolle fallbezogene Zusammenarbeit institutionell verankerte Arbeitszusammenhänge zu schaffen, in denen ggf. gemeinsam nach Wegen für eine bedarfsorientierte Beratung und Begleitung gesucht wird.

\subsection{Beispiele aus den Projektkommunen}

Die im Anhang ${ }^{56}$ wiedergegebenen Dokumente stellen Beispiele für interessante Varianten des Handlungskonzepts Case Management unter den Gegebenheiten des Feldes der Zuwanderung dar.

Der Zugang ins Case Management ist an den vier Standorten sehr unterschiedlich geregelt.

- In Dormagen sind die Mitarbeitenden im Case Management für den Erstkontakt mit allen Zugewanderten zuständig, der eventuell auch in Form eines Hausbesuchs stattfinden kann. Sie entscheiden dann selbst, wie intensiv die Zusammenarbeit erfolgen soll, d. h., ob Case Management durchgeführt wird oder nicht. Diese Vorgehensweise birgt das Problem in sich, dass die Entscheidungskriterien im Einzelfall im Dunkeln bleiben, es sei denn, es wird ein Reflexions- und Kontrollverfahren (z. B. in Form kollegialer Beratung) implementiert.

- In Köln erfolgt der Zugang über Meldungen verschiedener mit Zugewanderten arbeitenden Akteure (einschließlich Ehrenamt) an die Case Management-Steuerstelle bei der Kommune, die dann das weitere Verfahren einleitet. In diesen beiden Fällen existiert eine Schnittstelle zwischen dem Erstkontakt und dem Case Management, die eine Quelle für Konflikte sein kann, wenn es keine eindeutigen, für beide Stellen nachvollziehbaren Kriterien für den Übergang gibt. Eine entsprechende Qualifizierung des Personals in einer Anlaufstelle hinsichtlich der Inhalte von Case Management ist absolut sinnvoll, um Reibungsverluste zu vermeiden.

- Moers hat im Vergleich zu Dormagen den entgegengesetzten Weg beschritten und eine Servicestelle als Anlaufstelle für alle Zugewanderten eingerichtet. Im Zuge der Neuaufnahme erfolgt eine ausführliche Klä-

56 Der Anhang ist über folgenden Link abrufbar: https://www.nomos-shop.de/isbn/ 978-3-8487-7628-3. 
rung in Form eines offenen Gesprächs über die Lebenssituation einer Klientin bzw. eines Klienten. Die erhobenen Daten und Informationen dienen den Mitarbeitenden der Servicestelle, eine Entscheidung über weitere Unterstützungsformen zu treffen. Dazu gehören sowohl das eigene kommunale wie auch das Case Management-Angebot der Verbände, aber auch möglichst zeitgleiche niederschwellige Beratungs- und Unterstützungsangebote durch die Mitarbeitenden der Servicestelle.

- In Mülheim/Ruhr ist der Intake hochkomplex gestaltet, die (zukünftigen) Case Manager sind beteiligt. Bereits im „Vorverfahren“ werden Daten erhoben und Beratungsangebote unterbreitet, die auch genutzt werden können, wenn kein förmliches Case Management angeboten wird.

An den vier Standorten ist das Verhältnis des kommunalen Case Managements zu analogen Angeboten freier Träger sehr unterschiedlich.

- Diese Angebote spielen in den Konzepten der Stadt Mülheim/Ruhr und der Stadt Dormagen keine besondere Rolle, Case Management für Zugewanderte findet hier fast ausschließlich im kommunalen Kontext statt.

- Komplett anders stellt sich die Situation in Köln dar. Dort spielen die Verbände der freien Wohlfahrtspflege traditionell eine große Rolle. Die Stadt hat sich deshalb dafür entschieden, das gesamte operative Geschäft den Verbänden zu überlassen und hierzu kein eigenes Personal einzusetzen. Allerdings bleibt die strategische Steuerung bei der Kommune. Die jeweiligen Zuständigkeiten und Schnittstellen werden über entsprechende Vereinbarungen geregelt.

- Moers beschreitet hier einen Mittelweg. Analog zu Köln sind zunächst die Verbände am Zug; ist deren Case Management-Angebot jedoch ausgelastet, tritt das kommunale Case Management als „Deckungsreserve“ ein.

- Beide Lösungen, das Kölner wie das Moerser Konzept erfordern klare Absprachen und eine flexible Steuerungseinheit bei der Kommune.

Der Rechtskreiswechsel ins SGB II stellt eine Herausforderung für alle Case Management-Konzepte dar. Gesetzlich ist das Fallmanagement nach \$14 SGB II zuständig; allerdings können Aufgaben - ggf. im Kontext des $\$ 16$ a SGB II - an die Kommune übertragen werden. Köln und Moers setzen auf Absprachen mit dem Job Center, damit das einmal begonnene Case Management ggf. auch über den Rechtskreiswechsel hinaus weitergeführt werden kann - z. B. im Rahmen des $\mathbb{1} 16$ a SGB II. Mülheim ist dabei, diese Schnittstelle zu bearbeiten, es liegen aber zurzeit (31.12.2019) noch 
keine Ergebnisse vor. Die Stadt Dormagen hat ein „Schnittstellenpapier“ erarbeitet, in dem die Zuständigkeiten geklärt werden.

In allen vier Fällen handelt es sich um Projekte bzw. Konzepte - praktische Erfahrungen liegen nur in sehr begrenztem Umfang vor. 


\section{Fachliche Anforderungen an ein Monitoring des Integrationsmanagements für Geflüchtete und Neuzugewanderte ${ }^{57}$}

\subsection{Vorbemerkung}

„Monitoring“ ist derzeit fast ein Modebegriff. Monitor leitet sich vom lateinischen „monere“ ermahnen, warnen ab. Gerade in politischen und administrativen Prozessen verspricht man sich von Monitoringverfahren rechtzeitige Hinweise auf komplexe Vorgänge durch eine kontinuierliche Beobachtung und Darstellung in einem anvisierten Handlungsfeld. Die Wirtschaftswissenschaften ordnen das Monitoring als „Instrument zur Verringerung von Informationsasymmetrien" der strategischen Frühaufklärung im Rahmen des strategischen Managements zu. Wikipedia formuliert: „Monitoring ist die Überwachung von Vorgängen. Es ist ein Überbegriff für alle Arten von systematischen Erfassungen (Protokollierungen), Messungen oder Beobachtungen eines Vorgangs oder Prozesses mittels technischer Hilfsmittel oder anderer Beobachtungssysteme." Monitoring hat damit wesentlich geringere Leistungs- und Untersuchungstiefen als Prozesse des Sozialberichtswesens oder gar der Evaluation. Monitoring versammelt in der Regel quantifizierte Informationen aus kontinuierlich verfügbaren Datenbeständen. Monitoring liefert u. E. lediglich erste Hinweise und Signale aus den zur Beobachtung ausgewählten Vorgängen, bedarf aber zur Interpretation weiterer Analysen, Diskurse und Bewertungen. Monitoringdaten sind keineswegs für eine direkte Steuerung hinreichend. Filsinger definiert für die Bundeszentrale für politische Bildung Integrationsmonitoring wie folgt: „Monitoring bezeichnet die systematische und in regelmäßigen Abständen wiederholte Beobachtung, Beschreibung und Analyse von sozialen Sachverhalten mit Hilfe von Indikatoren. Indikatoren sind Kenngrößen, die über nicht oder nur schwer unmittelbar beobachtbare soziale Tatsachen Auskunft geben sollen. Sie erlauben eine genaue Beschreibung von Sachverhalten in Form von Messwerten und eine vergleichende Analyse zu vorab bestimmten bzw. zu früheren Messwerten. Mit den Integrationsmonitorings wird das Integrationsgeschehen (Integra-

57 Dieses Kapitel wurde von Heiner Brülle verfasst. 
tionsprozesse und Integrationsergebnisse) in einer längerfristigen Perspektive mit sozialwissenschaftlichen Methoden beobachtet. Erwartet werden können somit ein besseres Verständnis von Integrationsprozessen und Erkenntnisse zu den Wirkungen der Integrationspolitik. Dies wiederum soll eine rationale Integrationspolitik ermöglichen.“ (Filsinger 2019) Diese Definition ist insoweit zu präzisieren, dass Indikatoren keinen Sachverhalt unmittelbar messen, sondern lediglich einen komplexen Gegenstand wie z. B. Chancengleichheit in der Bildung - in der Regel theoriebasiert - über einen „Anzeigerwert" = Indikator oder besser mehrere Indikatoren als Indikatorenset abbilden. Ein Indikator kann so „als Messgröße, die Sachverhalte anzeigt, die nicht unmittelbar messbar sind" (Schubert/Klein 2018: S. 167), bezeichnet werden.

Die Analyse der Indikatoren kann unterschiedliche Zielrichtungen fokussieren: „über Vergleiche mit kritischen Schwellenwerten (Grenzwertperspektive), früheren Messwerten (Entwicklungsperspektive), ex-ante bestimmten Zielwerten (Zielerreichungsperspektive) oder den Ergebnissen anderer Beobachtungseinheiten (Bilanzierungsperspektive)" (Filsinger 2014: S. 10).

Wesentlich für ein Monitoring ist somit die Bildung der Kenngrößen bzw. Indikatoren und die klare Definition des Gegenstandbereiches, d. h. des Beobachtungsfeldes, der Definition von Personengruppen, deren Integration beobachtet und der Institutionen, deren Integrationsleistungen gemessen werden sollen.

In der Regel thematisiert das Integrationsmonitoring die große heterogene Kategorie der Menschen mit Migrationshintergrund, d. h. alle Menschen die unabhängig von ihrer Nationalität bzw. ihren Nationalitäten $\mathrm{Zu}-$ wanderungserfahrungen haben, d. h. im Ausland geboren sind und Personen, bei denen mindestens ein Elternteil außerhalb Deutschlands geboren ist und seit dem 1.1.1950 bzw. 1.1.1955 zugewandert ist, unabhängig ob sie in Deutschland oder noch im Ausland geboren sind. ${ }^{58}$ Abhängig von der Datenlage wird die Kategorie hinsichtlich der Merkmale Nicht-DeutscheStaatsangehörigkeit und/oder spezielle Aufenthaltsstatus differenziert.

58 Die Grenze 1950 liegt der Definition des Mikrozensus seit 2005 zugrunde, während die Grenze 1955 der Definition des Zensusgesetzes 2011 entstammt. 


\subsection{Das Integrationsmonitoring Nordrhein-Westfalen}

Das Integrationsmonitoring Nordrhein-Westfalen ist das zentrale Datenangebot zum Stand des Integrationsgeschehens in Nordrhein-Westfalen, es basiert auf gemeinsam festgelegten Indikatoren und Definitionen der Integrationsministerkonferenz. Diese veröffentlicht alle zwei Jahre einen Bericht zur Zuwanderung und zum Stand der Integration in den 16 Bundesländern. Gemeinsamkeiten und unterschiedliche Entwicklungstrends werden so dokumentiert.

Nordrhein-Westfalen leitet gemeinsam mit Berlin die länderoffene Arbeitsgruppe „Indikatorenentwicklung und Monitoring“, die den Bericht vorbereitet. Mit dem Gesetz zur Förderung der gesellschaftlichen Teilhabe und Integration in Nordrhein-Westfalen (Teilhabe- und Inte-grationsgesetz) vom 14. Februar 2012 ist die Integrationsberichterstattung in NRW auf eine gesetzliche Grundlage gestellt worden. In $₫ 15$ heißt es:

„(1) Die Landesregierung legt dem Landtag alle fünf Jahre einen Integrationsbericht vor, der die Bevölkerungsentwicklung unter besonderer Berücksichtigung der Zuwanderung (Zuwanderungsmonitoring), den Stand der Integration von Menschen mit Migrationshintergrund auf der Grundlage von Zielen und Indikatoren (Integrationsmonitoring) sowie die integrationspolitischen Maßnahmen und Leistungen des Landes in umfassender Weise dokumentiert und bewertet.

(2) Das Prinzip des Gender Mainstreaming ist sowohl bei der Erstellung von Statistiken wie auch im Kontext der Erarbeitung von Indikatoren nach $\$ 15$ (1) grundsätzlich zu beachten.

(3) Jährlich wird eine kommentierte Zuwanderungs- und Integrationsstatistik veröffentlicht.“

Ziel des Integrationsmonitorings und der Tools des angebotenen Portals ist „,...die Versachlichung der Diskussion über Zuwanderung und Integration“. Es sollen „zuverlässige Daten an die Hand gegeben werden, die eine vorurteilsfreie und sachgerechte Diskussion über den Stand der Integration unterschiedlicher Gruppen von Menschen mit Migrationshintergrund ermöglichen.“ Ferner wird darauf hingewiesen, dass „...das Instrument der Indikatorenberechnung nicht mit einer Ursachenanalyse gleichzusetzen" sei. Zudem wird bedauert, dass insbesondere Daten zu subjektiven Einstellungen und Meinungen fehlten und dass das Indikatorenset nicht abschließend sei, sondern bei gesicherter Datenbasis regelmäßig aktualisiert werden solle. „Der Vergleich auf der Zeitschiene erlaubt es, Fortschritte und Rückschritte der Integration zu erkennen“ (www. Integrati- 
onsmonitoring.nrw.de). Das statistische Landesamt erstellt jährliche kommentierte Integrationsstatistiken und ermöglicht Integrationsprofile der Kreise und kreisfreien Städte. Für alle Kreise und kreisfreien Städte in Nordrhein-Westfalen veröffentlicht das Land Integrationsprofile mit den Schwerpunkten Demografie, Bildung und Arbeit. Die jährlich aktualisierten Profile sollen verdeutlichen, wie sich Integration und Teilhabe auf kommunaler Ebene entwickeln. Der Anspruch ist es, ein wertvolles Arbeitsmittel für alle bereitzustellen, die kommunale Daten zur Integration und zur Lebenslage von Zugewanderten benötigen.

In Folgenden wollen wir die ambitionierte Zielsetzung überprüfen und insbesondere die Reichweite und Grenzen der dargestellten Indikatoren für eine Bewertung des Integrationsgeschehens diskutieren. Zunächst sollen die im Monitoring gewählten Datenquellen und die Indikatorenbildung referiert werden. Wesentliche Datenquellen sind der Mikrozensus und die amtliche Bevölkerungsfortschreibungen auf Basis des Zensus 2011, das Ausländerzentralregister (AZR), die Wanderungs- und Einbürgerungsstatistik, die Arbeitslosen und Beschäftigungsstatistik der Bundesagentur für Arbeit, die Kinder- und Jugendhilfestatistik und amtliche Schuldaten (ASD). Die Population der Zugewanderten wird in den dargestellten Integrationsprofilen wie folgt differenziert:

- Personen mit Migrationshintergrund gemäß der Erhebungsverordnung des Mikrozensus.

- Nicht-Deutsche wobei selbstverständlich auch Personen mit einer weiteren Staatsangehörigkeit als Deutsche zählen.

Zusätzlich können Eingebürgerte, Aussiedler, Aussiedlerinnen und Bevölkerung der jeweils zehn am stärksten vertretenen Nationalitäten ausgewiesen werden; in den verfügbaren kommunalen Integrationsprofilen werden diese Gruppen - vermutlich aufgrund kleiner Fallzahlen - jedoch nicht abgebildet. In der Konzeption des Monitorings wird ausgeführt, dass „wo immer möglich (...) Daten für Menschen mit und ohne Migrationshintergrund verwendet" werden und nur wenn dies nicht möglich sei, werde auf Daten für Ausländer und Ausländerinnen zurückgegriffen (vgl. www. Integrationsmonitoring.nrw.de). Der Stand der Integration soll für die kreisfreien Städte und Kreise in Nordrhein-Westfalen über folgendes Indikatorenset abgebildet werden:

- Demografische Indikatoren: Deutsche und Nicht-Deutsche nach Geschlecht und Alter, Bevölkerung mit Migrationshintergrund nach Geschlecht, die zehn am stärksten vertretenen Herkunftsländer der NichtDeutschen; $\mathrm{Zu}$ - und Fortzüge von Deutschen und Nicht-Deutschen aus dem/ins Ausland nach Geschlecht; Saldo der Zu- und Fortzüge der 
Deutschen und Nicht-Deutschen über die Grenzen Nordrhein-Westfalens nach Geschlecht.

- Rechtliche Integration: Ausländische Bevölkerung nach aufenthaltsrechtlichen Status (langfristiges Aufenthaltsrecht, befristete Aufenthaltserlaubnis, Duldung, Asylsuchende/Aufenthaltsgestattung, Sonstige/Ungeklärt) und Geschlecht.

- Arbeitsmarkt/Wirtschaft: Erwerbstätigenquote nach Migrationshintergrund und Geschlecht; Deutsche und Nicht-Deutsche sozialversicherungspflichtig Beschäftigte nach Geschlecht; Arbeitslosenquote der Deutschen und Nicht-Deutschen nach Geschlecht.

- Soziale Teilhabe: SGB II-Quote der Deutschen und Nicht-Deutschen.

- Bildung und Erziehung: Kinder in Kindertageseinrichtungen mit und ohne Migrationshintergrund differenziert nach Altersgruppen und Geschlecht; Kinder in Kindertageseinrichtungen nach Altersgruppen, Geschlecht und vorrangige Familiensprache Deutsch - Nicht-Deutsch; Neuaufnahmen deutscher und ausländischer Schüler und Schülerinnen in die 5.Klasse nach Schulformen und Geschlecht; Übergänge deutscher und ausländischer Schüler und Schülerinnen in die Sekundarstufe II; Schulabgänger und Schulabgängerinnen nach Deutsch Nicht-Deutsch, Geschlecht und Abschlussart.

Die Indikatorensets entsprechen der gängigen Verfahrensweise des Integrationsmonitorings in Deutschland, allerdings thematisieren sie nur das Integrationsgeschehen auf Seiten der Migrantinnen und Migranten und ignorieren das Integrationsgeschehen auf Seiten der Aufnahmegesellschaft. Diese Einseitigkeit ist zwar weitgehend durch die Datenlage begründet, aber wie insbesondere das Integrationsbarometer des Sachverständigenrats deutscher Stiftungen für Integration und Migration zeigt (vgl. Sachverständigenrat 2018b), lassen sich aus Befragungsdaten die Einstellungen und Erfahrungen zur Integration auf beiden Seiten kontinuierlich beobachten. Der Sachverständigenrat der Stiftungen veröffentlicht auf Umfragedaten basierende Indikatoren, die auch das Integrationsgeschehen seitens der Aufnahmegesellschaft abbilden. Um dieses Defizit des Indikatorensets des Statistischen Landesamtes zu kompensieren, sollte zumindest der jährliche Bericht und die Homepage mit einem Link zum Integrationsbarometer des Sachverständigenrats ergänzt werden.

Ein weiteres, gravierenderes Problem besteht darin, dass die Gruppen der Menschen mit Migrationshintergrund und der Ausländer und Ausländerinnen nicht differenziert werden können. In den Indikatorensets, die 
die Teilhabe ${ }^{59}$ thematisieren, stehen weder Differenzierungen hinsichtlich des Aufenthaltsstatus noch der Aufenthaltsdauer oder gar der sozialen Schichtung in Deutschland zur Verfügung. Eine Betrachtung der Großkategorien - als soziale Gruppe sollte man sie aufgrund ihrer großen Heterogenität nicht beschreiben - „Menschen mit Migrationshintergrund“ oder „Ausländer und Ausländerinnen“ über den zeitlichen Verlauf und/oder im Vergleich zu anderen Gebietskörperschaften gibt vor, das Integrationsbzw. besser das Teilhabegeschehen der Population wie z. B. Entwicklung der Gymnasialquote oder der Erwerbstätigenquote zu messen. Tatsächlich werden aber keine Veränderungen einer stabilen Population zu verschiedenen Zeitpunkten oder Gebietskörperschaften erfasst, sondern in erster Linie Veränderungen der sozialen Zusammensetzung der Populationen selbst. Wenn man die gravierenden Veränderungen der Population der Ausländer und Ausländerinnen zwischen 2014 und 2016 betrachtet, so zeigen die Indikatoren deutliche Rückgänge der Beteiligungsquoten im Bereich Bildung oder Erwerbstätigkeit, begründet sind diese durch die massive Zahl der Neuzugewanderten, insbesondere der Geflüchteten, die oft noch gar keine Beteiligungsmöglichkeiten wahrnehmen konnten. Die Großkategorien „Menschen mit Migrationshintergrund“ und „Ausländer und Ausländerinnen" können aufgrund ihrer Heterogenität die durch das diskontinuierliche Wanderungsgeschehen bedingten Veränderungen nicht adäquat erfassen. Für den genannten Zeitraum 2014-2016 signalisieren die Indikatoren eher Integrationsrückschritte, obwohl nach unseren Einschätzungen einerseits eher von Teilhabefortschritten der Eingewanderten, die länger in Deutschland leben, und andererseits von einer Zunahme der Neuzuwanderung (Geflüchtete und Menschen aus dem EU-Balkan), die kaum Zeit für Teilhabefortschritte hatten, auszugehen ist. Beide Prozesse sind unabhängig voneinander, überlagern sich und werden letztlich durch die Indikatoren z. B. des NRW-Integrationsmonitorings bis zur Unkenntlichkeit vermengt. ${ }^{60}$

59 Zur unterschiedlichen Konzeptualisierung des Integrations- und Teilhabebegriffs vgl. Abschnitt 3.1.

60 Beispielhaft kann man diesen Sachverhalt mit einer kleinen unveröffentlichten Analyse aus Wiesbaden dokumentieren: Das Integrationsmonitoring der Landeshauptstadt Wiesbaden zeigt einen Rückgang des Indikators „Anteil der einzuschulenden Kinder mit Migrationshintergrund mit einem Kindertagesstättenbesuch von mindestens 18 Monaten" von 2013 mit 93,2\% auf 2016 auf 90,1\%; gleichzeitig ist in diesem Zeitraum der Anteil der aus dem Ausland Neuzugewanderten Kinder im Kita-Alter von unter $2 \%$ auf deutlich über $4 \%$ angestiegen. 
Die Indikatoren des Integrationsmonitorings der Bundesländer bzw. des Landes Nordrhein-Westfalen geben zur Steuerung des Integrationsgeschehens lediglich einen groben Überblick und bieten deshalb zur Steuerung des Geschehens seitens der Kommunen keine hinreichende Grundlage. Dieser Sachverhalt ist nicht durch eine unzureichende Umsetzung begründet, sondern durch die Datenlage, die auf der Ebene der statistischen Landesämter keine besseren Indikatoren ermöglicht.

\subsection{Teilhabe und Integration als Prozess sozialer Dienstleistungen}

Für die Beobachtung des kommunalen Integrationsgeschehens mit dem Ziel, Informationen für die Steuerung zu gewinnen, ist wie oben ausgeführt das Integrationsmonitoring des Landes Nordrhein-Westfalen nicht hinreichend. Ergänzend werden deshalb zwei Strategien empfohlen, die konkrete Teilhabe der unterschiedlichen relevanten sozialen Gruppen zu beobachten:

- Die konkreten Angebote der Projekte des Kommunalen Integrationsmanagements und des Förderprogramms „Durchstarten in Ausbildung und Arbeit" benötigen selbstverständlich ein Berichtswesen bzw. Monitoring, welches die konkreten Leistungsprozesse und Daten der am Case Management Teilnehmenden kontinuierlich erhebt und berichtet. Das Ministerium für Kinder, Frauen, Familie und Integration (MKFFI) mit der Koordinierungsstelle der Kommunalen Integrationszentren und die Gesellschaft für innovatibe Beschäftigungsförderung (G.I.B.), eine landeseigene Gesellschaft des Landes Nordrhein-Westfalen, erarbeiten derzeit gemeinsam mit den Projektkommunen eine entsprechende Datenbank bzw. Excel-Datei, die wesentliche Merkmale der Teilnehmenden und des Case Management-Prozesses erfassen soll (G.I.B.-Onlinedatenbank). Diese Datenbank kann natürlich nur das Teilhabegeschehen der mit dem Teilhabemanagement bzw. dem Kommunalen Integrationsmanagement (KIM) unterstützten Personen dokumentieren. Wir werden unten zeigen, dass diese Daten notwendige, aber keine hinreichenden Grundlagen für ein Berichtswesen bzw. Monitoring des kommunalen Teilhabe und Integrationsgeschehen bieten, da sie naturgemäß nur über die Teilnehmenden und nicht über die

Diese in den letzten zwei Jahren neuzugewanderten Kinder hatten de facto gar keine Chance, eine Kita mindestens 18 Monate zu besuchen. 
Gruppe der Nicht-Teilnehmenden, die zu den anvisierten Zielgruppen gehören, berichten können.

- Um die soziale Lage und die Teilhabechancen der gesamten Zielgruppe berichten zu können - seien es die Neuzugewanderten, die Geflüchteten oder speziell die Geduldeten oder Bevölkerungsgruppen ohne Migrationserfahrung als Vergleichsgruppe - ist es erforderlich, auch Informationen über die Nicht-Teilnehmenden zu erheben, um insgesamt die Zielgruppenerreichung und die in der Regel wahrscheinlichen selektiven Effekte der Inanspruchnahme zu beobachten. Wie oben bereits angemerkt (vgl. Abschnitt 3.1), empfiehlt es sich auf die Teilhabe zu fokussieren anstatt auf die Integration, da das Geschehen auf Seiten der Aufnahmegesellschaft sich noch stärker statistisch verfügbaren Daten entzieht als das Geschehen seitens der Zuwandernden.

Die Förderung der Teilhabechancen und der Befähigung zur Teilhabe sind die wesentlichen Zielbereiche des Integrationsmanagements. Die Leistungsprozesse des Case Managements sowie der vermittelten sozialen Hilfen fallen unter die Kategorie der personenbezogenen sozialen Dienstleistungen, deren Produktion das folgende Schaubild ${ }^{61}$ dokumentiert:

61 Das Produktionsmodell (vgl. Brülle/Reis 1998; 2002) basiert auf Überlegungen von Naschold u.a. 1996. 
Schaubild 12: Produktionsmodell personenbezogener sozialer Dienstleistungen

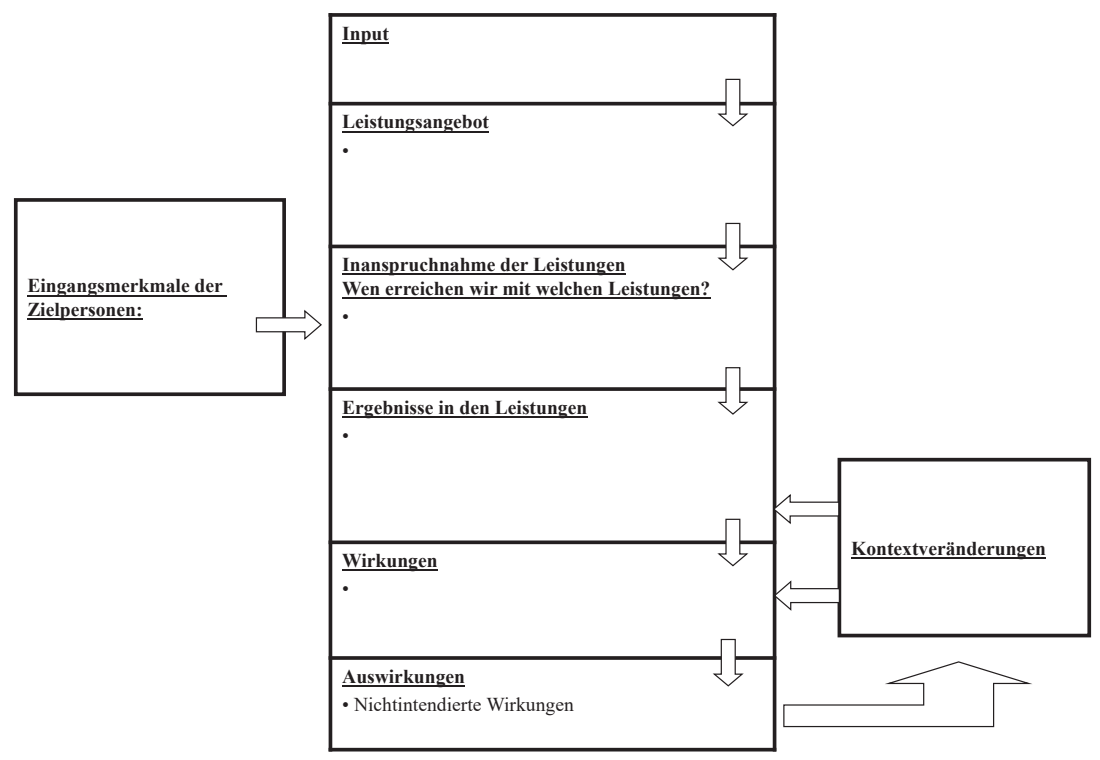

(Eigene Darstellung)

Das Schema ist deutlich vereinfacht, da insbesondere in Case Management-Prozessen (vgl. hierzu Kapitel 5) nicht ein einzelner Leistungsanbieter, sondern mehrere verschiedene Dienstleister koordiniert und möglicherweise auch unkoordiniert Leistungen anbieten und entsprechende Inanspruchnahmen erfahren. Für das Monitoring der Teilhabeprozesse interessiert insbesondere die anvisierte Zielgruppe, deren Inanspruchnahme oder Nicht-Inanspruchnahme einzelner Leistungen, sowie die Ergebnisse und Wirkungen der Leistungen. Für ein kontinuierliches Monitoring sind folgende Arbeitsschritte bzw. Fragen zu klären, die keineswegs vom Integrationsmanagement allein, sondern nur in den entwickelten Produktionsnetzwerken des kommunalen Integrationsmanagement (vgl. Kapitel 4) gemeinsam bearbeitet werden können:

- Input: Der eingesetzte Input von Geld, Personal oder sächliche Ressourcen ist an dieser Stelle nicht von Bedeutung.

- Leistungsangebot: Auch das verfügbare abstrakte Leistungsangebot kann hier außen vor bleiben.

- Eingangsmerkmale der Zielpersonen: Wer ist die Zielgruppe bzw. die zu beobachtende Grundgesamtheit? Eine klare Festlegung der Zielgruppe hinsichtlich sozialer Merkmale und - ggfls. geschätzter - An- 
zahl ist bereits bei der Problemanalyse und Planung der Leistungen unbedingt notwendig, aber auch um den wichtigen Aspekt der Nicht-Inanspruchnahme berücksichtigen zu können. Im Falle des Programms „Durchstarten in Ausbildung und Arbeit" 62 und des Kommunalen Integrationsmanagements des MKFFI sind die geduldeten Flüchtlinge zwischen 18 und 27 Jahre eindeutig benannt. Die Zahl und einige soziale Merkmale wie Alter, Geschlecht, Aufenthaltsstatus, Familienstand, Kinder etc. lassen sich aus dem Ausländerzentralregister für die jeweilige Kommune entnehmen. Eine eindeutige Zielgruppendefinition und deren quantitative Beschreibung sind damit möglich. Sinnvolle Vergleichsgruppen könnten alle Personen zwischen 18 und 27 Jahren oder alle aus dem Ausland Zugewanderten (z. B. in den letzten vier Jahren) sein. Für das Programm „Kommunales Integrationsmanagement", welches 2020 startet, ist die Zielgruppe wesentlich breiter angelegt; es wird insbesondere die seit ca. 2015 aus dem Ausland Neuzugewanderten fokussieren. Zur Ermittlung der Grundgesamtheit bietet es sich an, in der kommunalen Einwohnerstatistik alle Personen zu selektieren, die seit 1.1.2015 direkt aus dem Ausland oder aus einer Aufnahmeeinrichtung zugewandert sind.

- Inanspruchnahme der Leistungen: Welche Leistungen werden an welche Adressaten- und Adressatinnengruppen mit welchen Zielen angeboten und welche Adressaten- und Adressatinnnengruppen nehmen welche Leistung in Anspruch bzw. nutzen sie nicht? Bei den Leistungen bietet sich eine Differenzierung sowohl hinsichtlich der Case Management-Phasen (Intake, Erstberatung, Assessment, Zielvereinbarung, Fallabschluss) als auch hinsichtlich der zentralen Regelleistungen im Produktionsnetzwerk an (z. B. Kinderbetreuung, Sprachförderung, Berufliche Orientierung/Bildung etc.). An dieser Stelle wird der wichtige Zugang zur Teilhabe, mit anderen Worten die Teilhabechancen der Adressaten- und Adressatinnengruppen und ihre Möglichkeiten eine Teilhabe zu realisieren (Teilhabekompetenzen) beobachtet. Dies sind wesentliche Hürden, an denen Programme reüssieren oder scheitern können. Ich werde unten einzelne Leistungen und deren Monitoring beispielhaft darstellen. Verfahren der Datenerhebung, deren Weiterleitung und Auswertung sind in den jeweiligen Produktionsnetzwerken zu entwickeln und verbindlich zu vereinbaren.

62 Ein 2019 aufgelegtes gemeinsames Programm der Ministerien für Arbeit, Gesundheit und Soziales sowie Kinder, Familie, Flüchtlinge und Integration des Landes Nordrhein-Westfalen. 
- Ergebnisse in den Leistungen: Welche Adressaten- und Adressatinnengruppen erzielen welche Ergebnisse? Die zu beobachtenden Ergebnisse sind gemeinsam mit den Beteiligten im Produktionsnetzwerk als Indikatoren eindeutig zu definieren. Wesentliche Ergebnisformen sind z. B. kontinuierliche Teilnahmen bzw. Abbrüche, Prüfungsteilnahmen und -ergebnisse innerhalb des Leistungsprozesses etc.

- Wirkungen: Für welche Adressaten- und Adressatinnengruppen lassen sich im Anschluss an die Angebotsteilnehme welche Wirkungen feststellen? Auch erzielte Wirkungen sind vorab durch die beteiligten Leistungsanbieter als Indikatoren klar zu operationalisieren. Wirkungen sind im Sinne des Ko-Produktionsverhältnisses von Dienstleistenden und Nutzenden im Anschluss an die Teilnahme an einem Leistungsprozess zu erwarten. Typische Wirkungsindikatoren lassen hinsichtlich von Übergangsquoten und Einstiege oder Ausstiege bei den Regelsystemen operationalisieren, aber auch subjektive Zufriedenheits- oder Erfolgseinschätzungen der Teilhabenden sind relevante Wirksamkeitsindikatoren.

- Auswirkungen: Gibt es nicht-intendierte Auswirkungen der Teilhabeleistungen? Klassische nicht-intendierte Auswirkungen sozialer Dienstleistungsprozesse sind „Lock in“- oder Einsperreffekte, wenn z. B. die Teilnahme an einer arbeitsmarktpolitischen Leistung die frühe Einmündung in Erwerbsarbeit verhindert oder verzögert. Auch Stigmatisierungseffekte können zu den nicht-intendierten Auswirkungen gehören.

- Kontextveränderungen: Gibt es im Beobachtungszeitraum gravierende Kontextveränderungen, die für eine Interpretation der Monitoringergebnisse wesentlich sind? Auch diese Frage darf bei der Bewertung der Leistungsprozesse nicht unter den Tisch fallen, da während der Laufzeit der Dienstleistung sich z. B. sowohl die Eingangsqualitäten der Zielgruppen als auch die Übergangschancen oder die rechtlichen Regularien geändert haben können. Insbesondere aufenthalts- oder arbeitsrechtliche Regulierungen können erhebliche Effekte auf die Inanspruchnahme, Ergebnisse oder Wirkungen von Integrationsleistungen haben. 


\subsection{Wesentliche Felder sozialer Hilfen für das Integrationsmanagement für Geflüchtete und Neuzugewanderte}

Beim kommunalen Integrationsmanagement ${ }^{63}$ geht es nicht um die Konstruktion und Entwicklung spezieller Hilfen bzw. Hilfesysteme für die Zielgruppe der Migrantinnen und insbesondere der Neu-Eingewanderten. Die Leitbegriffe Teilhabe und Integration verweisen vielmehr auf die Regelsysteme sozialer Hilfen als Ort der Befähigung und Teilhabe. Kommunales Integrationsmanagement hat dabei die Aufgabe, möglichst alle Hilfebedürftigen aus der (bzw. den) Zielgruppe(n) zu erreichen und ihnen den Weg in geeignete Hilfen der Regelsysteme zu eröffnen und zu begleiten (vgl. Abschnitt 3.2). Gleichzeitig ist es die Aufgabe der kommunalen Koordinatoren und Koordinatorinnen und des kommunalen Integrationsmanagements, gemeinsam mit den verantwortlichen Akteuren und Akteurinnen der betroffenen Regelsysteme die teilhaberelevanten Felder sozialer Hilfe auf die neuen Zielgruppen und deren Bedarfe vorzubereiten. In den wesentlichen Feldern gilt es, Produktionsnetzwerke zu etablieren (vgl. Kapitel 4). Ein Berichtswesen und auf Daten gestütztes Monitoring der Inanspruchnahmen und Verläufe der Leistungsprozesse ist eine wesentliche Aufgabe der Akteure in den entsprechenden Produktionsnetzwerken oder Kooperationen.

Abschließend zeigen wir in folgender Tabelle 3 einige Möglichkeiten für die Dimensionierung und Operationalisierung der entsprechenden Monitoringprozesse.

63 Ich verwende den Begriff „Integrationsmanagement“, obwohl es im Wesentlichen um die Planung und Steuerung von Teilhabeprozessen geht, da der Begriff „Teilhabemanagement" für ein praktiziertes Verfahren in der NRW-Landesinitiative „Gemeinsam klappt's“ verwandt wird. 


\begin{tabular}{|c|c|c|c|c|c|}
\hline 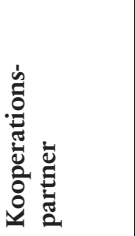 & 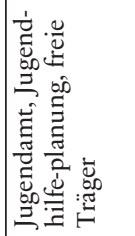 & 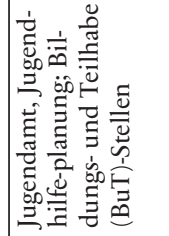 & 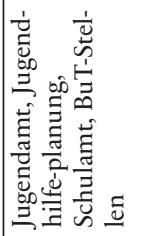 & 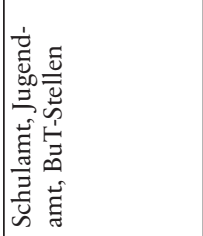 & 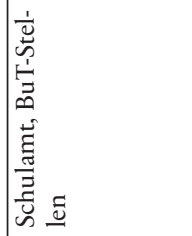 \\
\hline 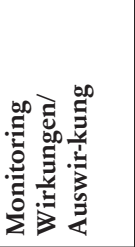 & 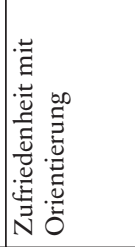 &  & 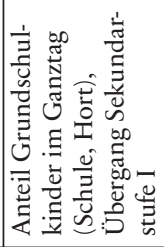 &  & 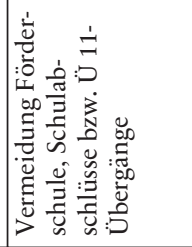 \\
\hline 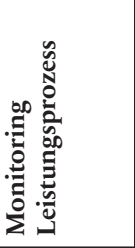 & 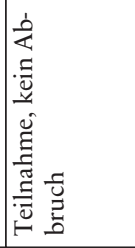 & 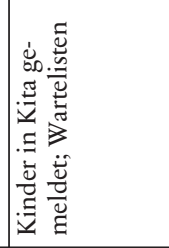 & 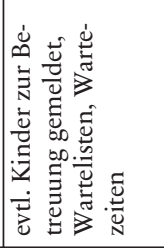 & 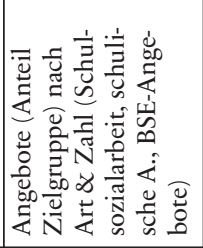 & 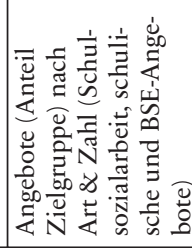 \\
\hline 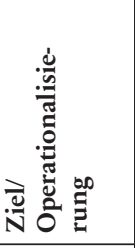 & 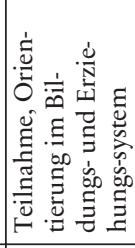 &  & 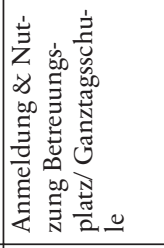 & 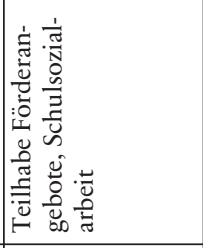 & 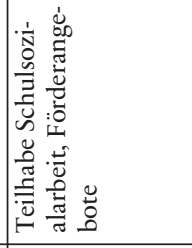 \\
\hline 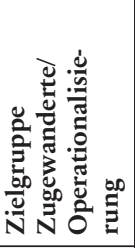 & 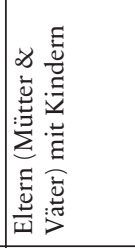 &  & 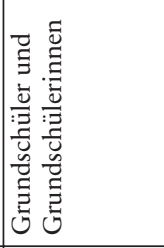 & 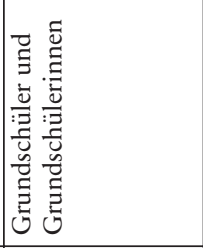 & 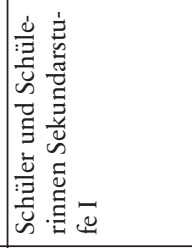 \\
\hline 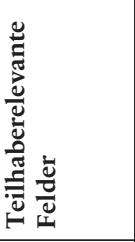 & 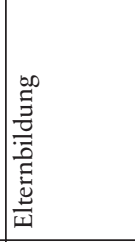 & 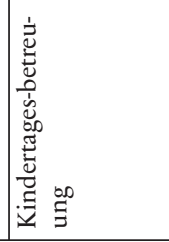 & 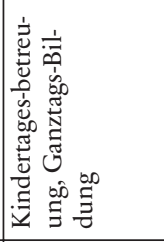 &  & 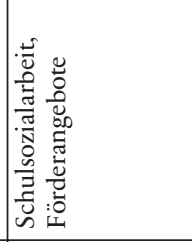 \\
\hline 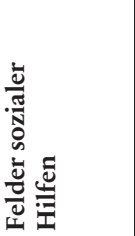 & 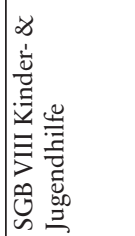 & & 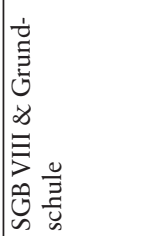 & 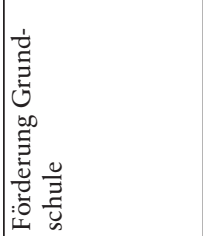 & 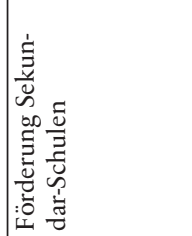 \\
\hline
\end{tabular}




\begin{tabular}{|c|c|c|c|}
\hline 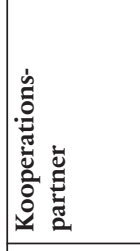 & 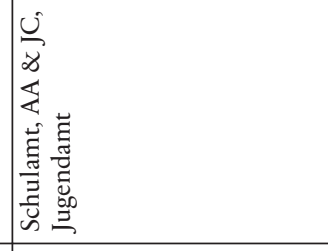 & 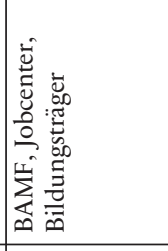 & $\begin{array}{l}0 \\
\\
\end{array}$ \\
\hline 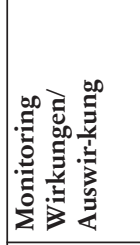 &  & 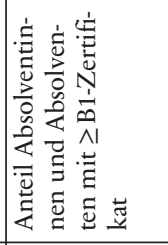 & 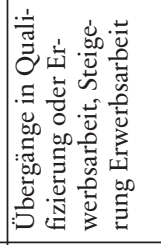 \\
\hline 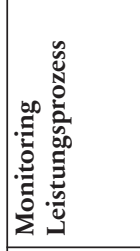 & 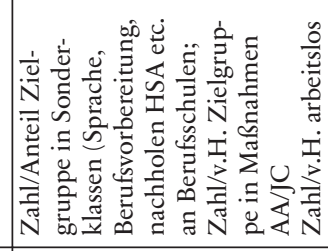 & 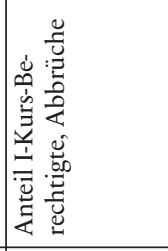 & 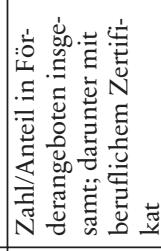 \\
\hline  & 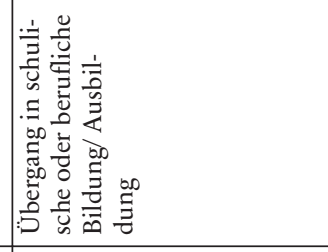 & 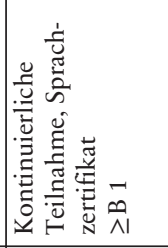 & 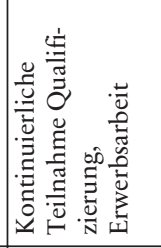 \\
\hline 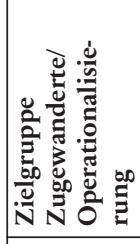 & 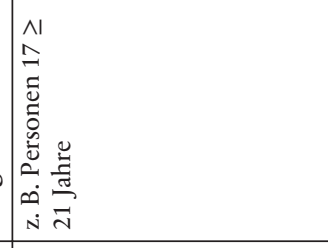 & 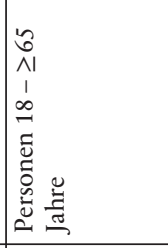 & 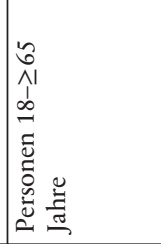 \\
\hline  & 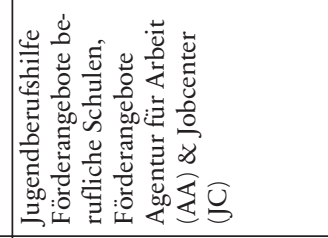 & 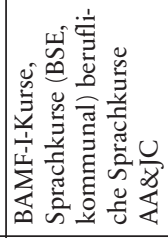 & 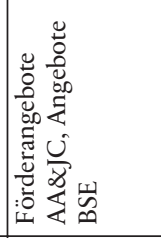 \\
\hline  & 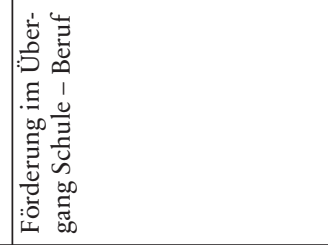 & 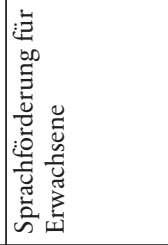 & 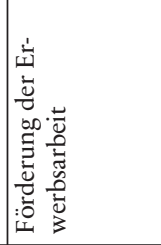 \\
\hline
\end{tabular}




\section{Literatur}

Alkemeyer, T./ Buschmann, N./ Michaeler, M. (2015): Kritik der Praxis. Plädoyer für eine subjektivierungstheoretische Erweiterung der Praxistheorien, in: Alkemeyer, T./Schürmann, V./Volbers, J. (Hg.): Praxis denken. Konzepte und Kritik. Wiesbaden, S. 25-50.

Alkemeyer, T./Buschmann, N. (2016): Praktiken der Subjektivierung - Subjektivierung als Praxis, in: Schäfer, H. (Hg.): Praxistheorie. Ein soziologisches Forschungsprogramm. Bielefeld, S. 115-136.

Alkemeyer, T./Buschmann, N. (2017): Befähigen. Praxistheoretische Überlegungen zur Subjektivierung von Mitspielfähigkeit, in: Rieger-Ladich, M./Grabau, C. (Hg.): Pierre Bourdieu: Pädagogische Lektüren. Wiesbaden, S. 271-297.

Allweyer, T. (2005): Geschäftsprozessmanagement, Herdecke u. a.

Argyris, Ch. (2002): Die lernende Organisation: Grundlagen, Methoden, Praxis, Stuttgart.

Aumüller, J./Bretl, C. (2008): Die kommunale Integration von Flüchtlingen in Deutschland, Berlin.

Badura, B./Gross, P. (1976): Sozialpolitische Perspektiven. Eine Einführung in Grundlagen und Probleme sozialer Dienstleistungen, München.

Baitsch, C./Müller, B. (2001): Moderation in regionalen Netzwerken, München u. a.

Balibar, E. (2010): Kommunismus und (Staats-)Bürgerschaft. Überlegungen zur emanzipatorischen Politik, in: Demirovic, A./Adolphs, St./Karakayali, S. (Hg.): Das Staatsverständnis von Nicos Poulantzas. Der Staat als gesellschaftliches Verhältnis, Baden-Baden, S. 19 - 34.

Ballew, J./Mink, G. (1996): Case Management in Social Work, Springfield.

Bartelheimer, P. (2007): Politik der Teilhabe: Ein soziologischer Beipackzettel, in: Fr.-Ebert-Stiftung (Hg.): Arbeitspapier Nr. 1/2007 des Projektes Gesellschaftliche Integration, Berlin.

Bartelheimer, P. (2009): Verwirklichungschancen als Maßstab lokaler Sozialpolitik? In: Sozialer Fortschritt 58 (3), S. 48-55.

Bauer, P. (2005): Institutionelle Netzwerke steuern und managen. Einführende Überlegungen, in: Bauer, P./Otto, U. (Hg.): Mit Netzwerken professionell zusammenarbeiten, Band II: Institutionelle Netzwerke in Steuerungs- und Kooperationsperspektive, Tübingen, S. 11-52.

Benz, A. (1995): Politiknetzwerke in der horizontalen Politikverflechtung, in: Jansen, D./Schubert, K. (Hg.): Netzwerke und Politikproduktion, Marburg, S. 185204. 
Bergmann, J. (2014): Der Fall als epistemisches Objekt, in: Bergmann, J./Dausendschön-Gay, U./Oberzauchner, F. (Hg.): „Der Fall“. Studien zur epistemischen Praxis professionellen Handelns, Bielefeld, S. 423-440.

Berlit, Uwe (2018a): Migration und ihre Folgen - wie kann das Recht Zuwanderung und Integration in Gesellschaft, Arbeitsmarkt und Sozialordnung steuern (Teil 1), in: ZAR 7/2018, S. 229-240.

Berlit, Uwe (2018b): Migration und ihre Folgen - wie kann das Recht Zuwanderung und Integration in Gesellschaft, Arbeitsmarkt und Sozialordnung steuern (Teil 2), in: ZAR 8/2018, S. 287-299.

Bernhard, St. (2010): Die Konstruktion von Inklusion. Europäische Sozialpolitik aus soziologischer Perspektive, Frankfurt am Main.

Bienzle, H./Gelabert, E./Jütte, W./Kolyva, K./Meyer, N./Tilkin, G. (2007): Die Kunst des Netzwerkens. Europäische Netzwerke im Bildungsbereich, Wien.

Böhm, V. (2017): "Fördern und Fordern" als Integrationskonzept - Anwendungsbereich, Systematik, Verfassungsrechtlicher Rahmen, in: Zeitschrift für Ausländerrecht und Ausländerpolitik (ZAR) 5-6/2017, S. 208-214.

Böhnisch, L./Lösch, H. (1973): Das Handlungsverständnis der Sozialarbeiter und seine institutionelle Determination, in: Otto, H./Schneider, S. (Hg.): Gesellschaftliche Perspektiven der Sozialarbeit. Neuwied u. a., S. 21-43.

Bommes, M. (1999): Migration und nationaler Wohlfahrtsstaat, Opladen/Wiesbaden.

Bommes, M. (2003): Die politische Verwaltung von Migranten in Gemeinden, in: Oltmer, J. (Hg.): Migration steuern und verwalten, Göttingen, S. 459-480.

Bommes, M. (2008): „Integration findet vor Ort statt“ - Über die Neugestaltung kommunaler Integrationspolitik, in: Bommes, M./Krüger-Potratz, M. (H.): Migrationsreport 2008, S. 159-194.

Bommes, M. (2018): Die Rolle der Kommunen in der bundesdeutschen Migrations- und Integrationspolitik, in: Gesemann, F./Roth, R. (Hg.): Handbuch lokale Integrationspolitik, Wiesbaden, S. 99-124.

Bommes, M./Tacke, V. (2006): Das Allgemeine und das Besondere des Netzwerkes, in: Hollstein, B./Strauss, F. (Hg.): Qualitative Netzwerkanalyse. Konzepte, Methoden, Anwendungen, Wiesbaden, S. 37-62.

Bommes, M.1/Scherr, A. (1996): Exklusionsvermeidung, Inklusionsvermittlung und/oder Exklusionsverwaltung. Zur gesellschaftstheoretischen Bestimmung sozialer Arbeit, in: neue praxis 2/1996, S. 107-123.

Bongaerts, G. (2008): Verdrängungen des Ökonomischen. Bourdieus Theorie der Moderne, Bielefeld.

Bonvin, J.-M./Farvaque, N. (2005): What Informational Basis for Assessing JobSeekers? Capabilities vs. Preferences, in: Review of Social economy 2/2005, S. 269-289.

Borkenhagen, P./Jäkel, L./Kummer, A./Megerle, A./Vollmer, L.-M. (2004): Netzwerkmanagement, Berlin.

Bourdieu, P. (2003): In Algerien, Zeugnisse der Entwurzelung, Graz. 
Bourdieu, P. (2006): Das Recht und die Umgehung des Rechts, in: Florian, M./ Hillebrandt, F. (Hg.): Pierre Bourdieu: Neue Perspektiven für die Soziologie der Wirtschaft, Wiesbaden, S. $73-108$.

Bourdieu, P. (2017): Über den Staat. Vorlesungen am College de France 19891992, Frankfurt am Main.

Bourdieu, P./Wacquant, L.J.D.(1996): Reflexive Anthropologie, Frankfurt am Main.

Brüggemann, H./Bremer, P. (2012): Grundlagen Qualitätsmanagement. Von den Werkzeugen über Methoden zum TQM, Wiesbaden.

Brühl, A. (2019): Was können Assessmentinstrumente leisten? In: Case Management 4/2019, S. 173-179.

Brülle, H./ Reis, C. (1998): Qualität in der sozialen Arbeit, in: Archiv für Wissenschaft und Praxis der sozialen Arbeit 4/1998 und 1/1999. S. 286-292.

Brülle, H./ Reis, C. (2002): Der sozialpolitische Kontext des Modellprojektes „Sozialagenturen - Hilfe aus einer Hand, in: MASQT: Sozialagenturen - Hilfe aus einer Hand. Düsseldorf, S. 33-61.

Buck, E. (2011): Asymmetrische Gemeinschaft. "Integration" als leerer Signifikant im Zugehörigkeitsdiskurs Deutschlands und Großbritanniens, in: Sociologica Internationalis, 1/2011, S. 29-52.

Bullinger, H.-J./Warnecke, H.-J. (Hg.) (1996): Neue Organisationsformen im Unternehmen. Ein Handbuch für das moderne Management, Berlin u. a.

Bundesagentur für Arbeit (2006): Interaktion zur Integration. Ein praxisorientierter Leitfaden zur Beratung im SGB II, Nürnberg.

Case Management Society of America (1995): Standards of Practice for Case Management. Little Rock.

Corbett, T./Noyes, J. (2004): Service and systems integration: A collaborative project, in: Focus 2/2004, S. 27-29.

Corbett, T./Dimas, J./Fong, J./Noyes, J. (2005): The challenge of institutional „milieu" to cross-systems integration, in: Focus 1/2005, S. 28-35.

Corbett, T./Noyes, J. (2006): Integrated Human Service Models: Assessing Implementation Fidelity Through the „Line of Sight“ Perspective, Milwaukee, http://1 egis.wisconsin.gov/lc/publications/rl/rl_2007-21.pdf (aufgerufen am 25.09.2009). Arbeitspapier.

Corbett, T./Noyes, J. (2008): Human Services Integration: A Conceptual Framework, Madison, Institute for Research on Poverty, Diskussionspapier Nr. 133308.

Danziger, S./Seefeldt, K. (2002): Barriers to employment and the "hard to serve“: Implications for services, sanctions, and time limits. In: Focus $1 / 2002$, Special Issue, www.ssw.umich.edu/poverty/foc221-part3-danziger.pdf (aufgerufen am 15.05.2004).

Denninger, E. (2001): Integration und Identität. Bitte um etwas Nachdenklichkeit, in: Kritische Justiz 4/2001, S. 442-452. 
Deutsche Gesellschaft für Care und Case Management (Hg.) (2015): Case Management Leitlinien - Rahmenempfehlungen, Standards und ethische Grundlagen. Heidelberg.

Deutsche Gesellschaft für Care und Case Management (Hg.) (2012): Was ist Case Management? https://www.dgcc.de/case-management (aufgerufen am 25.01.2019).

Deutscher Verein für öffentliche und private Fürsorge (2004): Empfehlungen des Deutschen Vereins zu Qualitätsstandards für das Fallmanagement, in: Nachrichtendienst des Deutschen Vereins (NDV), 5/2004, S. 149-158.

Deutscher Verein für öffentliche und private Fürsorge (2009): Anforderungen an das Fallmanagement im SGB II. Empfehlungen des Deutschen Vereins vom 17. Juni 2009, http://www.deutscher-verein.de/05-empfehlungen/empfehlungen_archiv/2009/p df/DV\%2001-09.pdf am 15.08.2009).

DiMaggio, P. J./Powell, W. W. (2009): Das “stahlharte Gehäuse” neu betrachtet. Institutioneller Isomorphismus und kollektive Rationalität in organisationalen Feldern, in: Koch, S. (Hg.): Neo-Institutionalismus in der Erziehungswissenschaft, Wiesbaden, S. 57-84.

Edwards, A./Daniels, H./Gallagher, T./Leadbetter, J./Warmington, P. (2009): Improving Inter-professional Collaborations. Multi-agency working for children's wellbeing, London and New York.

Ehlers, C./Müller, M./Schuster, F. (2017): Stärkenorientiertes Case Management. Komplexe Fälle in fünf Schritten bearbeiten. Opladen u. a.

Eichenhofer, J. (2013): Begriff und Konzept der Integration im Aufenthaltsgesetz, Baden-Baden.

Eichenhofer, J. (2016): Integrationsgesetzgebung, in: Zeitschrift für Ausländerrecht und Ausländerpolitik (ZAR) 8/2016, S. 251-263.

Eichenhofer, J./Dilmaghani, F. (2017): Mehr Integration und Teilhabe Zwei Vorschläge für rechtliche Neuregelungen, Berlin.

Engeström, Y. (2008a): Entwickelnde Arbeitsforschung. Die Tätigkeitstheorie in der Praxis, Berlin.

Engeström, Y. (2008b): Expansives Lernen in der Arbeitswelt: Für eine Neukonzeptionierung der Tätigkeitstheorie, in: Engeström, Y.: Entwickelnde Arbeitsforschung. Die Tätigkeitstheorie in der Praxis, Berlin, S. 61-90.

Engeström, Y. (2008c): Das Change Laboratory als Werkzeug zur Transformation der Arbeit, in: Engeström, Y.: Entwickelnde Arbeitsforschung. Die Tätigkeitstheorie in der Praxis, Berlin, S. 283-296.

Engeström, Y. (2008d): Gegenständlich orientiertes Inter-Agens: Für ein Verständnis kollektiver Intentionalität in verteilten Tätigkeitsfeldern, in: Engeström, Y. 2008: Entwickelnde Arbeitsforschung. Die Tätigkeitstheorie in der Praxis, Berlin, S. 91-118.

Eule, T. (2014): Inside immigration law: migration management and policy application in Germany, Farnham/Burlington. 
Eversberg, D. (2014): Dividuell aktiviert. Wie Arbeitsmarktpolitik Subjektivitäten produziert, Frankfurt am Main.

Ewers, M. (2000a): Case Management im Schatten von Managed Care: Sozial- und gesundheitspolitische Grundlagen, in: Ewers, M./Schaeffer, D. (Hg.): Case Management in Theorie und Praxis. Bern u. a., S. 30-53.

Ewers, M. (2000b): Das anglo-amerikanische Case Management: Konzeptionelle und methodische Grundlagen, in: Ewers, M./Schaeffer, D. (Hg.): Case Management in Theorie und Praxis. Bern u. a., S. 54-91.

Ewers, M./Schaeffer, D. (Hg.) (2000): Case Management in Theorie und Praxis. Bern u. a.

Filsinger, D. (2014): Monitoring und Evaluation. Perspektiven für die Integrationspolitik von Bund und Ländern, Friedrich-Ebert-Stiftung, Bonn.

Forschungsbereich beim Sachverständigenrat deutscher Stiftungen für Integration und Migration (2017): Wie gelingt Integration? Asylsuchende über ihre Lebenslagen und Teilhabeperspektiven in Deutschland, Berlin.

Foucault, M. (1986): Archäologie des Wissens. Suhrkamp, Frankfurt/Main.

Freire, P. (1991): Pädagogik der Unterdrückten. Bildung als Praxis der Freiheit, Reinbek.

Frings, D./Janda, C./Keßler, St./Steffen, E. (2018): Sozialrecht für Zuwanderer, Baden-Baden.

Fürst, D./Schubert, H. (1998): Regionale Akteursnetzwerke. Zur Rolle von Netzwerken in regionalen Umstrukturierungsprozessen, in: Raumforschung und Raumordnung. Spatial Research and Planning, 5/6/1998, Berlin u. a., S.352361.

Gaitanides, M. (1992): Ablauforganisation, in: Frese, E. (Hg.): Handwörterbuch der Organisation, Stuttgart, S. 1-18.

Gesellschaft für technische Zusammenarbeit (GTZ) (1987): ZOPP - Zielorientiertes Planen von Projekten und Programmen der technischen Zusammenarbeit (Einführung in die Grundlagen der Methode), GTZ-Form 21-23, Eschborn.

Giebeler, C. (2007): Perspektivenwechsel in der Fallarbeit und Fallanalyse, in: Giebeler, C./Fischer, W./Goblirsch, M./Miethe, I./Riemann, G. (Hg.): Fallverstehen und Fallstudien. Interdisziplinäre Beiträge zur rekonstruktiven Sozialarbeitsforschung, Opladen \& Farmington Hills, S. 9-22.

Greene, R. (1992): Case Management: An Area for Social Work Practice, in: Vourlekis, B./Greene, R. (Hg.): Social Work Case Management. New York, S. 11-25.

Groenemeyer, A. (2010): Doing Social Problems - Doing Social Control, in: Groenemeyer, A. (Hg.): Doing Social Problems: Mikroanalysen der Konstruktion sozialer Probleme in institutionellen Kontexten, Wiesbaden, S. 13 - 56.

Groß, Th. (2007): Das deutsche Integrationskonzept - vom Fördern zum Fordern?, in: ZAR 9/2007 S. 315-320.

Großmann, R./Lobnig, H./Scala, K. (2007): Kooperationen im Public Management. Theorie und Praxis erfolgreicher Organisationsentwicklung in Leistungsverbünden, Netzwerken und Fusionen, Weinheim u. a. 
Gusy, Ch./Müller, S. (2013): Leitbilder im Migrationsrecht, in: ZAR 8/2013, S. 265 -272 .

Hailbronner, K. (2017): Asyl- und Ausländerrecht, Stuttgart.

Hansbauer, P. (1996): „Mikrorationalitäten“ im Verwaltungsalltag: Dargestellt am Beispiel der „Hilfen zur Arbeit“ ( $\int \mathbb{S} 18 \mathrm{ff}$. BSHG) in einer Sozialverwaltung, in: Soziale Welt, 1/1996, S. 68-91.

Harrach, E.-M. von/Loer, T./Schmidtke, O. (2000): Verwaltung des Sozialen. Formen der subjektiven Bewältigung eines Strukturkonflikts. Konstanz.

Hasenfeld, Y. (1983): Human Service Organizations, Englewood Cliffs

Hasenfeld, Y. (Hg.) (2010): Human Services As Complex Organizations, Newbury Park u. a.

Häußermann, H./Kapphan, A. (2008): Integrationspolitik der Städte - ein Paradigmenwechsel, in: Bommes, M./Krüger-Potratz, M. (H.): Migrationsreport 2008, S. 15-48.

Hess, S./Lebuhn, H. (2014): Politiken der Bürgerschaft. Zur Forschungsdebatte um Migration, Stadt und citizenship, in: sublurban. zeitschrift für kritische stadtforschung, 3/2014, S. 11-34.

Hild, P. (1997): Netzwerke der lokalen Arbeitsmarktpolitik. Steuerungsprobleme in theoretischer und empirischer Sicht, Berlin.

Hillebrandt, F. (2002): Hilfe als Funktionssystem für Soziale Arbeit, in: Thole, W. (Hg.): Grundriss Soziale Arbeit. Ein einführendes Handbuch, Opladen, S. 215226.

Hirsch-Kreinsen, H. (2002): Unternehmensnetzwerke - revisited, in: Zeitschrift für Soziologie, 2/2002, S. 106-124.

Holstein, J.A./Miller, G. (2003): Social Constructionism and Social Problems Work, in: dies. (ed): Challenges and Choices. Constructionist Perspectives on Social Problems, New York, S. 70-91.

Hörster, R. (2010): Kasuistik, in: Bock, K./Miethe, I. (Hg.): Handbuch qualitative Methoden in der Sozialen Arbeit, Opladen \& Farmington Hills, S. 377-386.

Huke, N. (2010): Diskursive Formationen und Kräfteverhältnisse in der europäischen Migrationspolitik, Forschungsgruppe Europäische Integration, Studie Nr. 29, Marburg.

Huxham, C./Vangen, S. (2005): Managing to Collaborate. The theory and practice of collaborative advantage, London u. a.

Janotta, L.(2018): Inklusionsbegehren und Integrationsappelle: Aufenthalt, Soziale Arbeit und der Nationalstaat, in: neue praxis 2/2018, S. 122-143.

Kardorff, E. von (1998): Kooperation, Koordination und Vernetzung. Anmerkungen zur Schnittstellenproblematik in der psychosozialen Versorgung, in: Röhrle, B./Sommer, G./Nestmann, F. (Hg.): Netzwerkintervention. Fortschritte der Gemeindepsychologie und Gesundheitsförderung, Band 2, Tübingen, S. 203222.

Kaufmann, F.-X. (2002): Sozialpolitik und Sozialstaat: Soziologische Analysen, Opladen. 
Kaufmann, F.-X. (2012): Konzept und Formen sozialer Intervention, in: Albrecht, G./Groenemeyer, A. (Hg.): Handbuch soziale Probleme, Wiesbaden, S. 12851304.

Kluth, W. (2018): Migration und ihre Folgen- Wie kann das Recht Zuwanderung und Integration regulieren? in: NJW-Beilage 2018 S. $46-50$.

Köhling, K./Stöbe-Blossey, S. (2018): Kooperation von Akteuren vorbeugender Sozialpolitik. Eine Analyse am Beispiel der Berufsorientierung jugendlicher Flüchtlinge, Forschungsinstitut für gesellschaftliche Weiterentwicklung, Düsseldorf.

Kohlmeyer, K./Mauruszat, R./Seyfried, E. (2000): Lokale und regionale Netzwerke in der GI Beschäftigung, Berlin, Forschungsstelle für Berufsbildung, Arbeitsmarkt und Evaluation, Band 24, Diskussionspapier.

Kötter, U. (2016): EU-Sozialbürgerschaft oder Menschenrecht auf Gewährleistung eine menschenwürdigen Existenzminimums? Zur aktuellen Diskussion um das Recht von UnionsbürgerInnen auf soziale Grundsicherung in Deutschland, in: Sartorius, W./Weth H.-U.: Rechtsstaat, Markt und Menschenwürde, Freiburg im Breisgau, S 43-71.

Laclau, E./Mouffe, Ch. (2015): Hegemonie und radikale Demokratie. Zur Dekonstruktion des Marxismus. Wien.

Lamb, G./Stempel, J. (2000): Pflegerisches Case Management aus Patientensicht: die Entwicklung zum Insider-Experten, in: Ewers, M./Schaeffer, D.: Case Management in Theorie und Praxis. Bern u. a., S. 162-179.

Lebuhn, H. (2013): Migration - Recht - Citizenship. Poentiale und Grenzen eines kritischen Diskurses, in: Mecheril, P.: Migrationsforschung als Kritik? Wiesbaden, S. 231-244.

Lerch, F./Sydow, J./Wilhelm, M. (2007): Wenn Wettbewerber zu Kooperationspartnern (gemacht) werden. Einsichten aus zwei Netzwerken in einem Cluster optischer Technologien, in: Schreyögg, G./Sydow, J. (Hg.): Kooperation und Konkurrenz, Reihe Managementforschung, Band 17, Wiesbaden, S. 207-255.

Leven, B. (2018): Zu (des-)integrativen Wirkungen des rechts - Oder: (keine) Integration auf bayerisch: „Mia san (ned) mia! In: ZAR 9 /2018, S. 339-345.

Liebelt, W. (1992): Ablauforganisation, Methoden und Techniken, in: Frese, E. (Hg.): Handwörterbuch der Organisation, Stuttgart, S. 19-34.

Lindsay, C./McQuaid, R./Dutton, M. (2008): Inter-agency Cooperation and New Approaches to Employability, in: Social Policy \& Administration 7/2008, S. 715-732.

Lipsky, M. (1980): Street-Level Bureaucracy. Dilemmas of the Individual in Public Services, New York.

Löcherbach, P./Klug, W./Remmel-Faßbender, R./Wendt, W. (Hg.) (2018): Case Management. Fall- und Systemsteuerung in der Sozialen Arbeit. München.

Löcherbach, P./Mennemann, H./Hermsen, T. (Hg.) (2009): Case Management in der Jugendhilfe. München. 


\section{Literatur}

Ludwig, M. (2013): Kooperation im kommunalen Netzwerk. Das Beispiel der kommunalen Eingliederungsleistungen nach $\$ 16$ a SGB II, Manuskript, Frankfurt am Main.

Luhmann, N. (2000): Organisation und Entscheidung, Opladen.

Maihofer, W./Behrendt, R. F. (1967): Die Würde des Menschen, Hannover.

Maiwald, K.-O. (2008): Die Fallperspektive in der professionellen Praxis und ihrer reflexiven Selbstvergewisserung. Allgemeine Überlegungen und ein empirisches Beispiel aus der Familienmediation, in: Forum Qualitative Sozialforschung. 9/2008.

Marchart, O. (2017): Die Diskursanalyse der Essex School. Modell und Methode, in: Marchart, O. (Hg.): Ordnungen des Politischen, Staat - Souveränität - Nation, Wiesbaden.

Mezzadra, S./Neilson, B. (2013): Grenzen der Gerechtigkeit, Differentielle Inklusion und Kämpfe der Grenze, in: Heimeshoff, L.-M./ Hess, S./Kron, St/Schwenken, H./Trzeciak, M. (Hg.): Grenzregime II, Hamburg, S. 232-255.

Miebach, B. (2009): Prozesstheorie. Analysen, Organisation und System, Wiesbaden.

Milner, J./O’Byrne, P. (2002): Assessment in Social Work. London.

Ministerium für Arbeit und Soziales, Qualifikation und Technologie des Landes Nordrhein-Westfalen (2000): Modellprojekt „Sozialbüros“ NRW, Endbericht, Düsseldorf.

Ministerium für Wirtschaft und Arbeit des Landes Nordrhein-Westfalen (2003): Case Management. Theorie und Praxis, Düsseldorf.

Moebius, St. (2018): Diskurs - Ereignis - Subjekt. Diskurs- und Handlungstheorie im Ausgang einer poststrukturalistischen Sozialwissenschaft, in: Keller, R./ Hirseland, A./Schneider, W./Viehöver, W. (Hg.): Die diskursive Konstruktion von Wirklichkeit, Köln 2018.

Monzer, M. (2013): Case Management. Grundlagen, Heidelberg.

Morschett, D. (2005): Formen von Kooperationen, Allianzen und Netzwerken, in: Zentes, J./Swoboda, B./Morschett, D. (Hg.): Kooperationen, Allianzen und Netzwerke, Wiesbaden, S. 377-404.

Moxley, D. (1997): Case Management by Design, New York.

Müller, H.-P. (1992): Sozialstruktur und Lebensstile. Der neuere theoretische Diskurs über soziale Ungleichheit, Frankfurt/Main 1992.

Müller, H.-P. (2014): Pierre Bourdieu. Eine systematische Einführung, Berlin.

Naschold, F./Budäus, D./Jann, W./ Mezger, E./ Oppen, M./Picot, A./ Reichard, C./ Schanze, E./ Simon, N. (1996): Leistungstiefe im öffentlichen Sektor. Erfahrungen, Konzepte, Methoden, Berlin.

Naujoks, H. (1994): Konzernmanagement durch Kontextsteuerung. Die Relevanz eines gesellschaftstheoretischen Steuerungskonzepts für betriebswirtschaftliche Anwendungen, in: Schreyögg, G./Conrad, P. (Hg.): Dramaturgie des Managements. Laterale Steuerung. Reihe Managementforschung, Band 4, Berlin, S. 105-141. 
Negt, O. (2016): Überlebensglück. Eine autobiographische Spurensuche, Göttingen 2016.

Neumann, V. (2010): Ein neues Grundrecht für die Armen, in: vorgänge 1/2010, S. $102-110$.

Nida-Rümelin, J. (2017): Über Grenzen denken, Hamburg.

Nieswand, B. (2018): Problematisierung und Emergenz. Die Regimeperspektive in der Migrationsforschung, in: Pott, A./Rass, Ch./Wolff, F. (Hg.): Was ist ein Migrationsregime? Wiesbaden, S. 81-106.

Nonhoff, M. (2006): Politischer Diskurs und Hegemonie. Das Projekt „Soziale Marktwirtschaft", Bielefeld.

Oltmer, J. (2018): Deutsche Migrationsverhältnisse im europäischen Kontext seit dem 2. Weltkrieg, in: Gesemann, F./Roth, R. (Hg.): Handbuch lokale Integrationspolitik, Wiesbaden, S. 57 - 74.

Oltmer, J. (2018a): Einführung, in: Oltmer, J. (Hg.): Migrationsregime vor Ort und lokales Aushandeln von Migration, Wiesbaden, S. 1-12.

Oltmer, J. (2018b): Migration aushandeln. Perspektiven aus der Historischen Migrationsforschung, in: Pott, A./Rass, Ch./Wolff, F. (Hg.): Was ist ein Migrationsregime? Wiesbaden, S. 239-254.

Oostrik, H./Steenbergen, B. (2000): Case Management in der ambulanten psychosozialen Gesundheitsversorgung in den Niederlanden: das Nijmeger Modell, in: Ewers, M./Schaeffer, D.: Case Management in Theorie und Praxis. Bern u. a., S. 252-265.

Oppen, M. (1995): Qualitätsmanagement. Grundverständnisse, Umsetzungsstrategien und ein Erfolgsbericht: Die Krankenkassen. Berlin.

Opratko, B. (2012): Ein theoretischer Universalschlüssel? Zur Ontologisierung des Hegemoniebegriffs bei Laclau und Mouffe. In: Dzudzek, I./Kunze, C./Wullweber, J. (Hg.): Diskurs und Hegemonie. Gesellschaftskritische Perspektiven, Bielefeld, S. 59-84.

Payer, H. (2002): Wieviel Organisation braucht das Netzwerk? Entwicklung und Steuerung Reis, C./Schu, M./Brülle, H. (2009): Aktivierung in der Sozialhilfe (SGB XII). Abschlussbericht im Auftrag des Ministeriums für Arbeit, Gesundheit und Soziales des Landes

Nordrhein-Westfalen, Frankfurt am Main.

von Organisationsnetzwerken mit Fallstudien aus der Cluster- und Regionalentwicklung (Dissertation), Klagenfurt.

Pott, A./Rass, Ch./Wolff, F. (Hg.) (2018): Was ist ein Migrationsregime? Wiesbaden.

Rat für Migration (2017): Manifest für eine zukunftsfähige Migrations-, Flüchtlings- und Integrationspolitik, Berlin.

Reckwitz, A. (2006): Ernesto Laclau. Diskurse, Hegemonien, Antagonismen, in: Moebius, St./Quadflieg, D. (Hg.): Kultur. Theorien der Gegenwart, Wiesbaden, S. 339-349. 
Reis, C./Schu, M./Brülle, H. (2009): Aktivierung in der Sozialhilfe (SGB XII). Abschlussbericht im Auftrag des Ministeriums für Arbeit, Gesundheit und Soziales des Landes

Nordrhein-Westfalen, Frankfurt am Main.

Reis, C. /Hobusch, T./Kolbe, C. (2011): Fallmanagement im SGB II und in der Hilfe zum Lebensunterhalt (SGB XII). Ein kritischer Leitfaden, Frankfurt am Main.

Reis, C./Siebenhaar, B. (2015): Befähigen statt Aktivieren. Aktueller Reformbedarf bei Zielsetzung und Aufgabenstellung im SGB II, Friedrich-Ebert-Stiftung, WISO Diskurs 7/2015, Bonn.

Reis, C./Geideck, S./Hobusch, T./Schu, M./Siebenhaar, B./Wende, L. (2016): Produktionsnetzwerke in der lokalen Sozial- und Arbeitsmarktpolitik. Aufbau kooperativer Strukturen und Weiterentwicklung sozialer Dienstleistungen am Beispiel des SGB II, Frankfurt am Main.

Ring, P./Van de Ven, A. (1994): Developmental Processes of Cooperative Interorganizational Relationships, in: Academy of Management Review 1/1994, S. 90 118.

Sachverständigenrat deutscher Stiftungen für Integration und Migration (SVR) (2010), Einwanderung gestalten, Jahresgutachten 2010, Berlin.

Sachverständigenrat deutscher Stiftungen für Integration und Migration (SVR) (2018a), Steuern, was zu steuern ist. Was können Einwanderungs- und Integrationsgesetze leisten? Jahresgutachten 2018, Berlin.

Sachverständigenrat deutscher Stiftungen für Integration und Migration (SVR) (2018b): Integration in Nordrhein-Westfalen. Sonderauswertung des SVR-Integrationsbarometers 2018, Berlin. https://www.svr-migration.de/wp-content/uplo ads/2019/03/SVR-FB_Integrationsbarometer-NRW.pdf(Zugriff 15.01.2020).

Sandfort, J. (2010): Human Service Organizational Technology. Improving Understanding and Advancing Research, in: Hasenfeld, Y. (Hg.): Human Services As Complex Organizations, 2. Auflage. Newbury Park u. a., S. 269-290.

Santen, E. van/Seckinger, M. (2003): Kooperation: Mythos und Realität einer Praxis. Eine empirische Studie zur interinstitutionellen Zusammenarbeit am Beispiel der Kinder- und Jugendhilfe, München.

Santen, E. van/Seckinger, M. (2005): Fallstricke im Beziehungsgeflecht. Die Doppelebenen interinstitutioneller Netzwerke, in: Bauer, P./Otto, U. (Hg.): Mit Netzwerken professionell zusammenarbeiten, Band II: Institutionelle Netzwerke in Steuerungs- und Kooperationsperspektive, Tübingen, S. 202-219.

Schammann, H. (2017): Stadt, Land, Flucht. Konzeptionelle Überlegungen zum Vergleich städtischer Flüchtlingspolitik in Deutschland, in: Barbehön, M./ Münch, S.: Variationen des Städtischen - Variationen lokaler Politik, Wiesbaden, S. 91-117.

Schammann, H./Kühn, B. (2017): Kommunale Flüchtlingspolitik in Deutschland, Friedrich-Ebert-Stiftung, Berlin.

Scheffer, Th. (2014): Geschichten zur Fallarbeit, in: Bergmann, J.R./DausendschönGay, U./Oberzauchner. F. (Hg.): „Der Fall“. Studien zur epistemischen Praxis professionellen Handelns, Bielefeld, S. 225-248. 
Schiersmann, C./Thiel, H.-U. (2014): Organisationsentwicklung. Prinzipien und Strategien von Veränderungsprozessen, Wiesbaden.

Schmid, M./Schu, M./Vogt, I. (2012): Motivational Case Management. Ein Manual für die Drogen- und Suchthilfe. Heidelberg.

Schmidt, L./Haasse, R. (2010): Der Arbeitsbegriff in der Soziologie sozialer Probleme und im Neo-Institutionalismus, in: Groenemeyer, A. (Hg.): Doing Social Problems: Mikroanalysen der Konstruktion sozialer Probleme in institutionellen Kontexten, Wiesbaden, S. 57 - 78.

Schmidt-Wellenburg, Ch. (2013): Die Regierung des Unternehmens. Managementberatung im neoliberalen Kapitalismus, Konstanz und München.

Schneider, K. (2011): Assimilation und Integration - eine Begriffsanalyse aus der Perspektive der Rechtswissenschaft, in: ZAR 1/2011, S. 8-16.

Schneider, W. (2009): Grundlagen der soziologischen Theorie, Band 3: Sinnverstehen und Intersubjektivität - Hermeneutik, funktionale Analyse, Konversationsanalyse und Systemtheorie, Wiesbaden.

Schubert, H. (2005): Das Management von Akteursnetzwerken im Sozialraum, in: Bauer, P./Otto, U. (Hg.): Mit Netzwerken professionell zusammenarbeiten, Band II: Institutionelle Netzwerke in Steuerungs- und Kooperationsperspektive, Tübingen, S. 73-104.

Schubert, H. (2008): Netzwerkkooperation - Organisation und Koordination von professionellen Vernetzungen, in: Schubert, H. (Hg.): Netzwerkmanagement. Koordination von professionellen Vernetzungen - Grundlagen und Praxisbeispiele, Wiesbaden, S. 7-105.

Schubert, H. (2011): Kooperation, in: Deutscher Verein für öffentliche und private Fürsorge (Hg.): Fachlexikon der sozialen Arbeit, Baden-Baden, S. 531-533.

Schubert, K./Klein, M. (2018): Das Politiklexikon. Stichwort „Indikator“, Bonn.

Schulte, A. (2012): Politische Steuerung von Integrationsprozessen und Menschenrechte in der Einwanderungsgesellschaft, in :ZAR 8/2012, S. 289 - 297.

Scott, W. R. (2003): Organizations. Rational, Natural, and Open Systems. 5. Auflage, Upper Saddle River, New Jersey.

Sen, A. (2000): Ökonomie für den Menschen: Wege zur Gerechtigkeit und Solidarität in der Marktwirtschaft. München.

Steinbach, R. (2014): $₫ 1$ SGB I, in: Hauck, K./Noftz, W. (Hg.): Sozialgesetzbuch (SGB) I, Berlin.

Strassheim, H./Oppen, M. (2006): Lernen in Städtenetzwerken. Kooperation Konflikte - Kompetenzentwicklung, Berlin

Sydow, J. (1999): Management von Netzwerkorganisationen - Zum Stand der Forschung, in: Sydow, J. (Hg.): Management von Netzwerkorganisationen, Wiesbaden, S. 279-314.

Sydow, J./Windeler, A. (1997): Komplexität und Reflexivität, in: Ahlemeyer, H./ Königswieser, R. (Hg.): Komplexität managen, Wiesbaden, S. 147-162. 
Sydow, J./Windeler, A. (2000): Steuerung von und in Netzwerken - Perspektiven, Konzepte, vor allem aber offene Fragen, in: Sydow, J./Windeler, A. (Hg.): Steuerung von Netzwerken. Konzepte und Praktiken, Opladen, S. 1-24.

Sydow, J./Wirth, C./Manning, S. (2002): Autonomie und Bindung in Projektnetzwerken aus betriebswirtschaftlicher Perspektive. Literaturüberblick und erste konzeptionelle Überlegungen, Berlin, Arbeitspapier.

Sydow, J./Möllering, G. (2009): Produktion in Netzwerken. Make, Buy \& Cooperate, München.

Tacke, V. (2000): Netzwerk und Adresse, in: Soziale Systeme. Zeitschrift für soziologische Theorie. 2/2000, S. 291-320.

Thompson, T./Mikelson, K. (2001): Screening and Assessment in TANF/Welfare-toWork, http://www.urban.org/pdfs/screening_and_assessment_TANF-WtW.pdf (aufgerufen am 15.05.2004).

Thym, D. (2017): Migrationsfolgenrecht, in: Grenzüberschreitungen, Veröffentlichungen der Vereinigung der Deutschen Staatsrechtslehrer 76, Berlin/Boston, S. 169- 216,.

Van Berkel, R./Valkenburg, B. (2007): Making it personal. Individualizing activation services in the EU, Bristol.

van Riet, N./Wouters, H. (2002): Case Management, Luzern.

Wacquant, L. (2017): Mit Bourdieu in die Stadt: Relevanz, Prinzipien, Anwendungen, in: sub/urban. Zeitschrift für kritische Stadtforschung, 1-2/2017, S. 173196.

Wahrendorf, V. (2018): $\mathbb{1}$ SGB XII, in: Grube, Ch./Wahrendorf, V. (Hg.): SGB XII: Kommentar, München.

Wallerath, M. (2008): Zur Dogmatik eines Rechts auf Sicherung des Existenzminimums, Juristenzeitung(JZ), S. $157-168$.

Weisser, G. (1956): Wirtschaft, in: Ziegenfuß, W. (Hg.): Handbuch der Soziologie. Stuttgart.

Wendt, W. (1991): Unterstützung fallweise. Case Management in der Sozialarbeit. Freiburg i. Br.

Wendt, W. (1997): Case Management im Sozial- und Gesundheitswesen. Eine Einführung. Freiburg i. Br.

Wendt, W. (2005): Case Management. Stand und Positionen in der Bundesrepublik, in: Löcherbach, P./Klug, W./Remmel-Faßbender, R./Wendt, W. (Hg.): Case Management. Fall- und Systemsteuerung in der Sozialen Arbeit. München, S. 14-39.

Wernet, A. (2006): Hermeneutik - Kasuistik - Fallverstehen, Stuttgart.

Weth, H.-U. (2016): Rechtsstaat, Markt - und die Menschenwürde?!, In Sartorius, W./Weth, H.-U. (Hg): Rechtsstaat, Markt und Menschenwürde, S. 13 - 20, Freiburg im Breisgau.

Wilhelm, R. (2007): Prozessorganisation, München.

Windeler, A. (2001): Unternehmungsnetzwerke. Konstitution und Strukturation, Wiesbaden 
Wirth, C. (2007): Projektnetzwerke in der Arbeitsvermittlung: Eine Organisationsform der Zukunft? In: Arbeit. Zeitschrift für Arbeitsforschung, Arbeitsgestaltung und Arbeitspolitik 1/2007, S. 23-35.

Wissert, M. (2001): Unterstützungsmanagement als Rehabilitations- und Integrationskonzept bei der ambulanten Versorgung älterer, behinderter Menschen, Aachen.

Wullweber, J. (2012): Konturen eines politischen Analyserahmens - Hegemonie, Diskurs und Antagonismus, in: Dzudzek, I./Kunze, C./Wullweber, J. (Hg.): Diskurs und Hegemonie. Gesellschaftskritische Perspektiven. Bielefeld, S. 29-58.

Zacher, H. F. (1987): Das soziale Staatsziel, in: Isensee, J./Kirchhof, P.: Handbuch des Staatsrechts der Bundesrepublik Deutschland, Heidelberg, S. 1045

Zander, K. (2000): Case Management, klinische Pfade und CareMaps: Stand der Entwicklung und Diskussion in den USA, in: Ewers, M./Schaeffer, D. (Hg.): Case Management in Theorie und Praxis. Bern u. a., S. 92-117.

Internetquellen:

http://www.integrationsmonitoring.nrw.de/integrationsberichterstattung_nrw/Inte gration_kommunal/Integrationsprofile/index.php (Zugriff 16.1.2020)

http://www.integrationsmonitoring.nrw.de/integrationsberichterstattung_nrw/ziel setzung/index.php (Zugriff 16.1.2020)

http://www.integrationsmonitoring.nrw.de/integrationsberichterstattung_nrw/Gru ndlagen/Konzeption/index.php (Zugriff 16.1.2020)

Filsinger, D. (2019): Integrationsmonitoring. Kurzdossier für die Bundeszentrale für politische Bildung. http://www.bpb.de/gesellschaft/migration/kurzdossiers/2 91809/integrationsmonitoring? $\mathrm{p}=$ all (Zugriff 15.01.2020).

Wikipedia „Monitoring“ (Zugriff am 15.01.2020)

https://wirtschaftslexikon.gabler.de/definition/strategische-fruehaufklaerung-43088/ version-266421 (Zugriff am 15.01.2020) 


\section{Anhang}

Im Anhang werden die Case Management-Konzepte aus Dormagen (Anlage 1), Köln (Anlage 2), Moers (Anlage 3) und Mülheim (Anlage 4) vorgestellt. Es handelt sich um die Wiedergabe der von den Kommunen bzw. Vertreterinnen derselben erarbeiteten Konzepte.

Die Umsetzung von Case Management definiert häufig das Verhältnis $\mathrm{zu}$ anderen Organisationseinheiten auf kommunaler Ebene (Jugendamt, Jobcenter), zur Agentur für Arbeit und den Trägern der freien Wohlfahrtspflege neu. Deshalb ist es sinnvoll, entsprechend Zuständigkeiten und Schnittstellen festzulegen und ggf. Kooperationsvereinbarungen abzuschließen. Als Beispiele hierfür sind der das Jugendamt betreffende und bereits vorliegende Teil des „Schnittstellenpapiers“ der Stadt Dormagen (Anlage 5), sowie der Kooperations- und Weiterleitungsvertrag zwischen der Stadt Köln und Trägern der freien Wohlfahrtspflege (Anlage 6) wiedergegeben. Letzterer betrifft zwar das Teilhabemanagement im Rahmen der Initiativen „Gemeinsam klappt's“ des Ministeriums für Kinder, Familie, Flüchtlinge und Integration und „Durchstarten in Ausbildung und Arbeit" der Ministerien für Arbeit, Gesundheit und Soziales sowie für Kinder, Familie, Flüchtlinge und Integration, kann aber analog auf das Case Management im Rahmen des Kommunalen Integrationsmanagements übertragen werden.

Die Dokumente werden hinsichtlich Inhalt und Formatierung im Original wiedergegeben. 
